\title{
Vías y entrvías : atributos y compromisos del distrito industrial de Tarragona
}

Juan Manuel Zaguirre Fernández

ADVERTIMENT La consulta d'aquesta tesi queda condicionada a l'acceptació de les següents condicions d'ús: La difusió d'aquesta tesi per mitjà del repositori institucional UPCommons (http://upcommons.upc.edu/tesis) i el repositori cooperatiu TDX ( http://www.tdx.cat/) ha estat autoritzada pels titulars dels drets de propietat intel-lectual únicament per a usos privats emmarcats en activitats d'investigació i docència. No s'autoritza la seva reproducció amb finalitats de lucre ni la seva difusió i posada a disposició des d'un lloc aliè al servei UPCommons o TDX. No s'autoritza la presentació del seu contingut en una finestra o marc aliè a UPCommons (framing). Aquesta reserva de drets afecta tant al resum de presentació de la tesi com als seus continguts. En la utilització o cita de parts de la tesi és obligat indicar el nom de la persona autora.

ADVERTENCIA La consulta de esta tesis queda condicionada a la aceptación de las siguientes condiciones de uso: La difusión de esta tesis por medio del repositorio institucional UPCommons (http://upcommons.upc.edu/tesis) y el repositorio cooperativo TDR (http://www.tdx.cat/?localeattribute=es) ha sido autorizada por los titulares de los derechos de propiedad intelectual únicamente para usos privados enmarcados en actividades de investigación y docencia. No se autoriza su reproducción con finalidades de lucro ni su difusión y puesta a disposición desde un sitio ajeno al servicio UPCommons No se autoriza la presentación de su contenido en una ventana o marco ajeno a UPCommons (framing). Esta reserva de derechos afecta tanto al resumen de presentación de la tesis como a sus contenidos. En la utilización o cita de partes de la tesis es obligado indicar el nombre de la persona autora.

WARNING On having consulted this thesis you're accepting the following use conditions: Spreading this thesis by the institutional repository UPCommons (http://upcommons.upc.edu/tesis) and the cooperative repository TDX (http://www.tdx.cat/?localeattribute=en) has been authorized by the titular of the intellectual property rights only for private uses placed in investigation and teaching activities. Reproduction with lucrative aims is not authorized neither its spreading nor availability from a site foreign to the UPCommons service. Introducing its content in a window or frame foreign to the UPCommons service is not authorized (framing). These rights affect to the presentation summary of the thesis as well as to its contents. In the using or citation of parts of the thesis it's obliged to indicate the name of the author. 


\section{VÍAS Y ENTREVÍAS}

Atributos y compromisos del distrito industrial de Tarragona

TESIS DOCTORAL LIBRO III

Juan Manuel Zaguirre Fernández

Director: Josep Parcerisa i Bundó Catedrático de Urbanística 


\section{I983-2010}

\section{DOS CARAS DE LA MISMA MONEDA}

"El boom industrial de Tarragona, iniciado en los años 6o, supuso la llegada a la ciudad de miles de inmigrantes, que trabajaron en el montaje de las factorías químicas. Pero cuando, al final de los años 7o, finaliza la expansión, el trabajo de las constructoras y de las empresas de montaje empieza a disminuir, comporta que se tengan que desplazar a otras zonas. "Después de dos décadas de crecimiento constante, comienza la fase de recesión del mercado laboral a causa de dos factores. De un lado, las empresas del sector petroleoquímico requieren de un número bastante reducido de obreros no cualificados, mientras que técnicos y obreros especializados vienen de fuera, de otras plantas industriales de las mismas empresas". De otro lado, miles de inmigrantes participaron en la construcción de la petroleoquímica, muchos de los cuales, una vez acabada, han tenido que buscar trabajo en otros lugares. "El desarrollo industrial generaba pocos puestos de trabajo y, en su mayoría, de alta calificación". Las empresas químicas y petroleoquímicas son intensivas en capital, pero no en puestos de trabajo."

Extracto del libro, Els barris d'Adigsa. Campclar. R. M. ${ }^{a}$ Bosch, Barcelona: Generalitat de Catalunya, I995. 


\section{CARTOGRAFÍA Y DOCUMENTACIÓN}

\section{INDUSTRIALIZACIÓN Y CRECIMIENTO URBANO}

"En definitiva, toda la industrialización de Tarragona puede contemplarse en un trayecto en coche desde la Plaza Imperial Tárraco hasta el municipio de Vilaseca; a la izquierda de dicha ruta se ubican las empresas y, significativamente, a la derecha de la misma encontramos diez de los doce barrios obreros que estudiamos en nuestro trabajo.

[...] es difícil pensar cómo y en qué medida se habría producido el crecimiento urbano de Tarragona sin la triple iniciativa derivada de la creación de los polígonos industriales, el Plan de Estabilización y la ubicación en Tarragona de la refinería de petróleos de Cataluña. Lo que sí es indudable es que el fenómeno social del "nuevo urbanismo" de la periferia de Tarragona es un resultado directo de la industrialización de la ciudad. Existe, asimismo, una correspondencia directa entre las fases del proceso industrializador y las fases del crecimiento de los barrios. Existe, por decirlo así, un barrio para cada fase de la implantación industrial."

Extracto del libro, Los Barrios de Tarragona: una aproximación antropológica. PUJADAs, J.; BARDAjí, F. Tarragona: Ayuntamiento, I987.

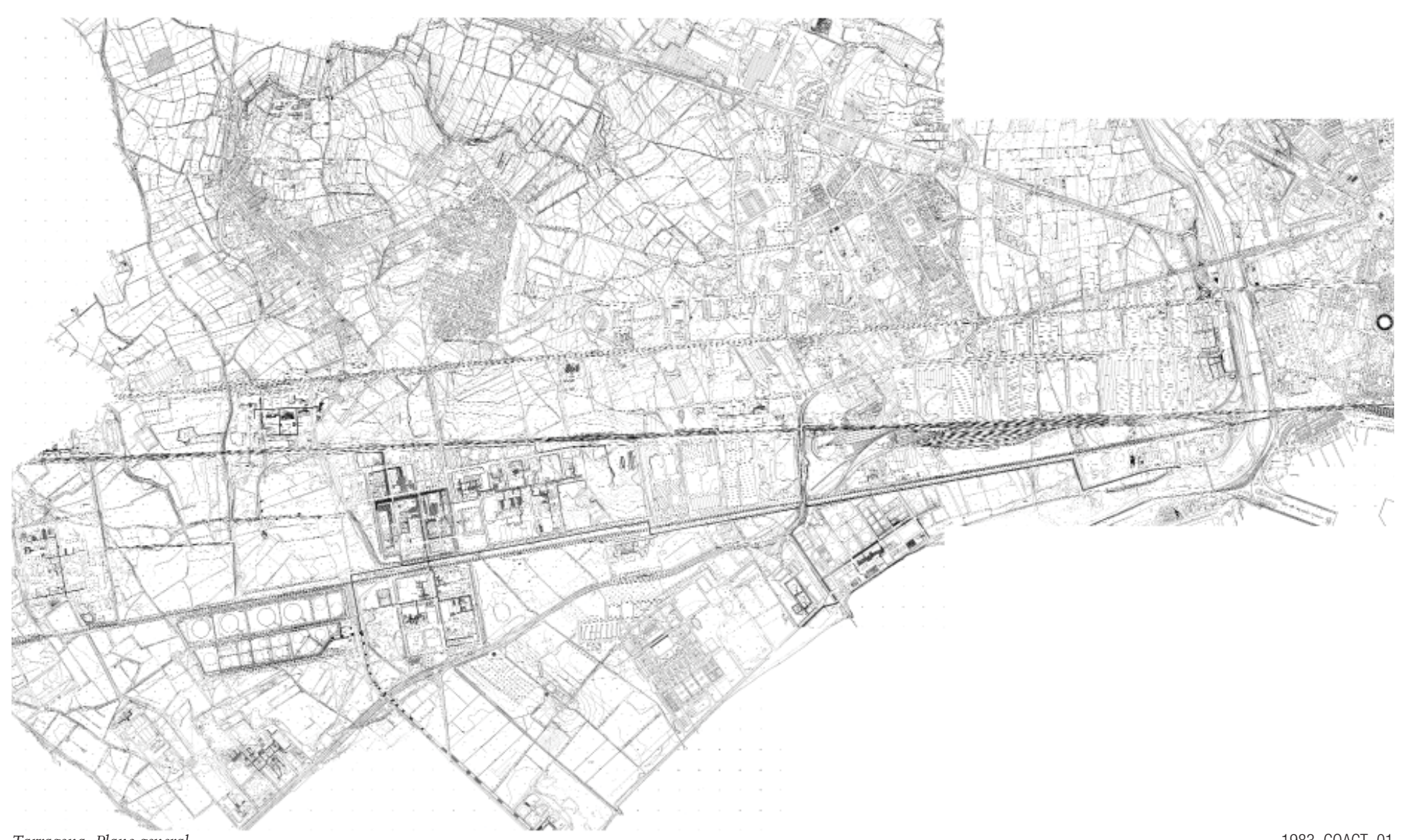



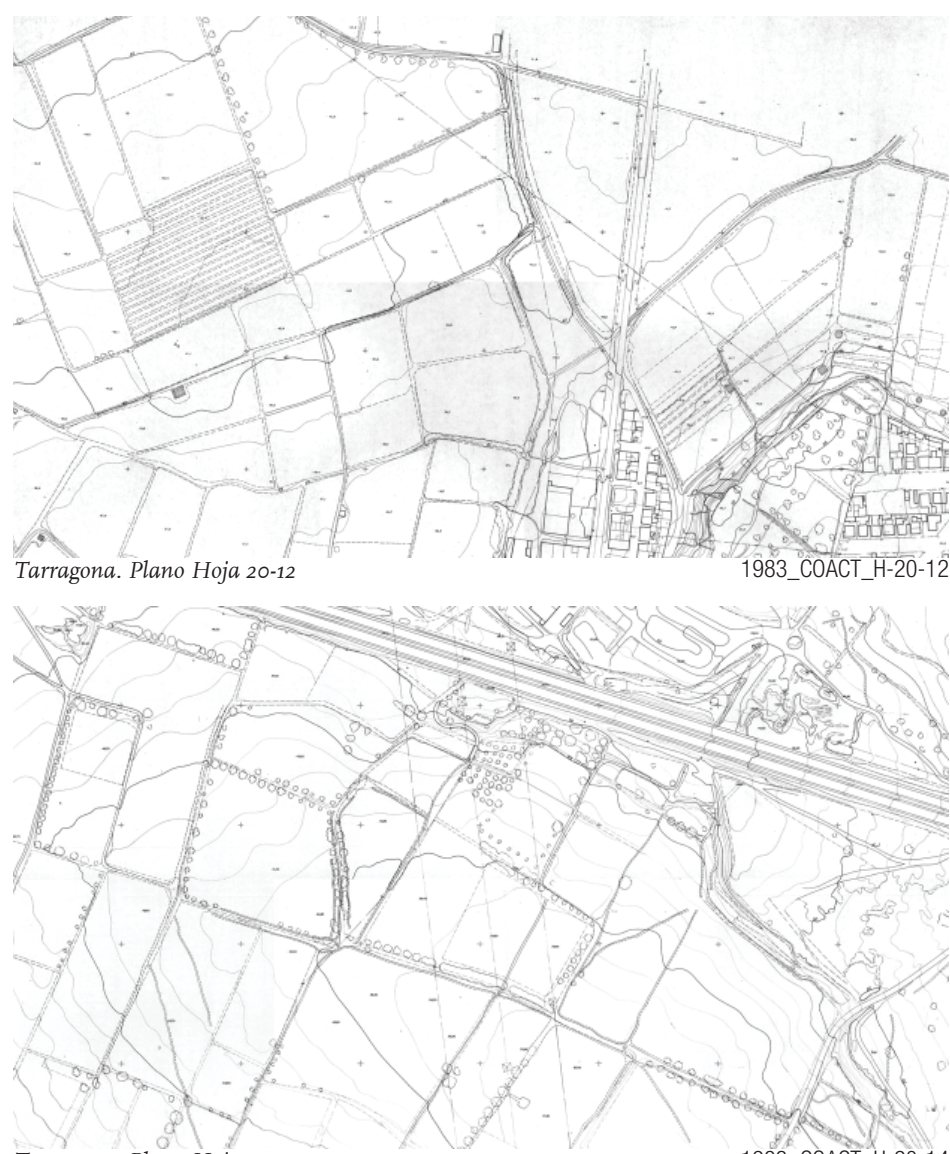

Tarragona. Plano Hoja 20-14

1983 COACT H-20-14

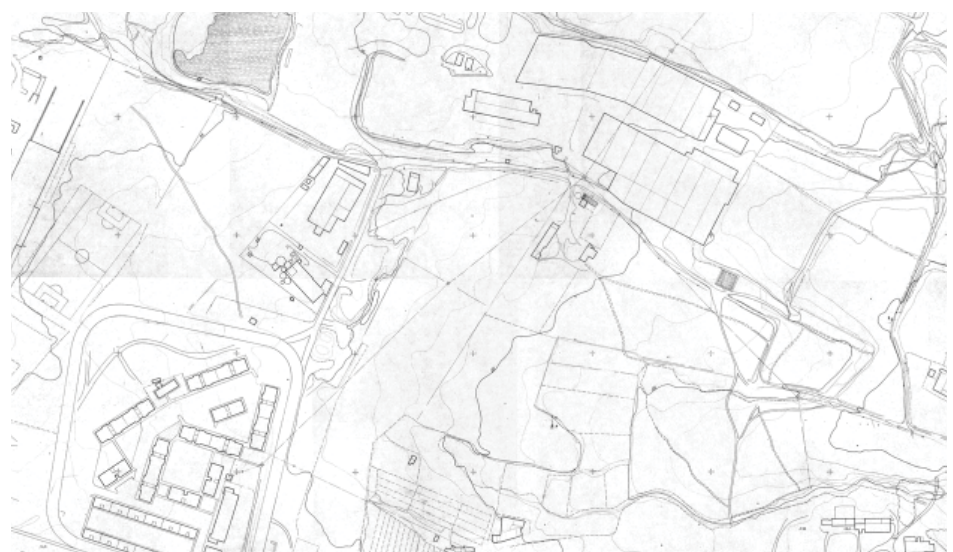

Tarragona. Plano Hoja 20-16

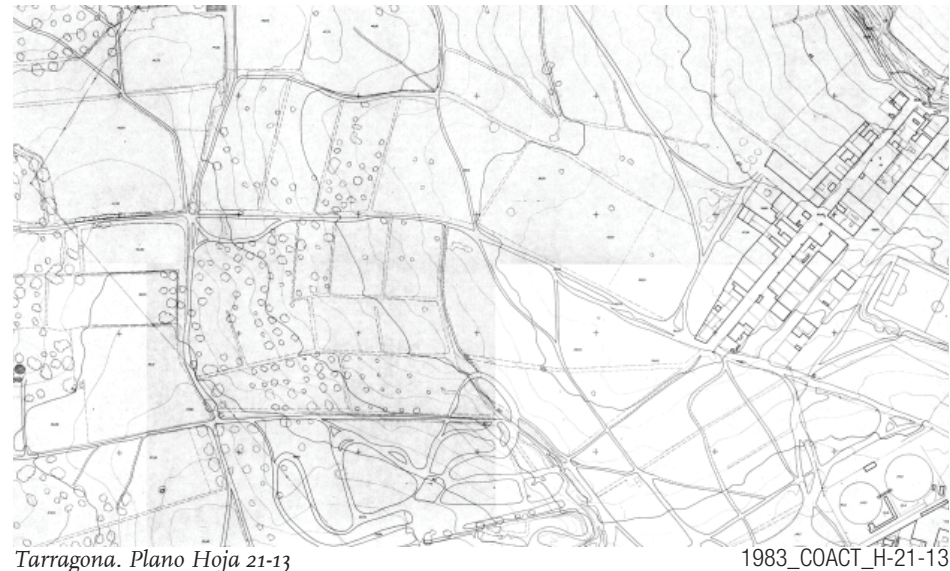

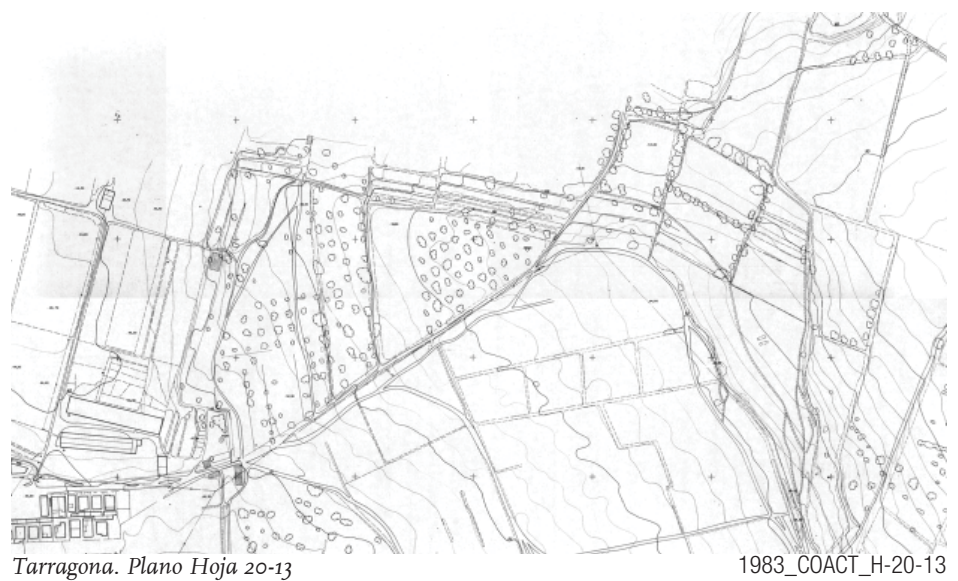
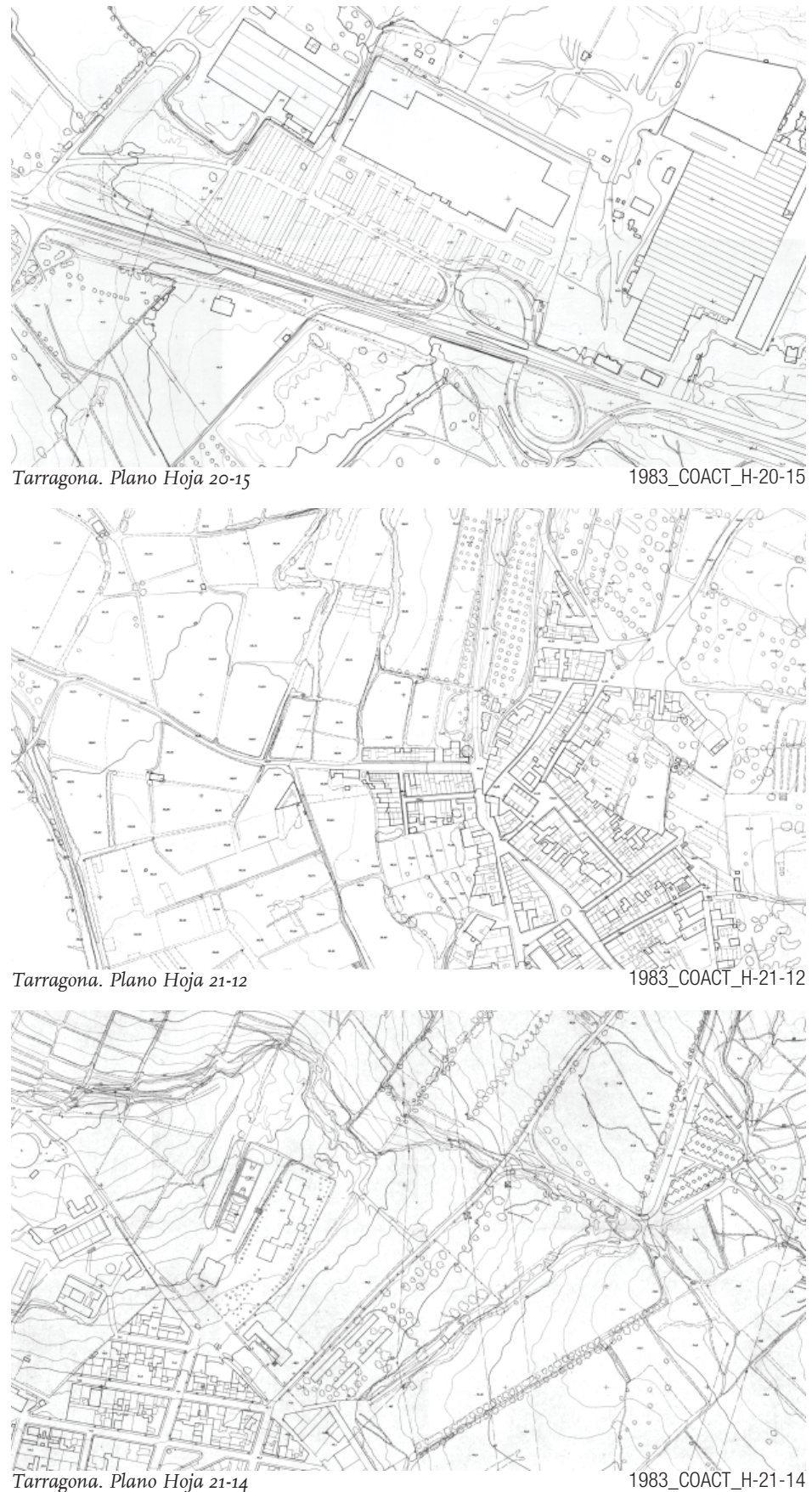


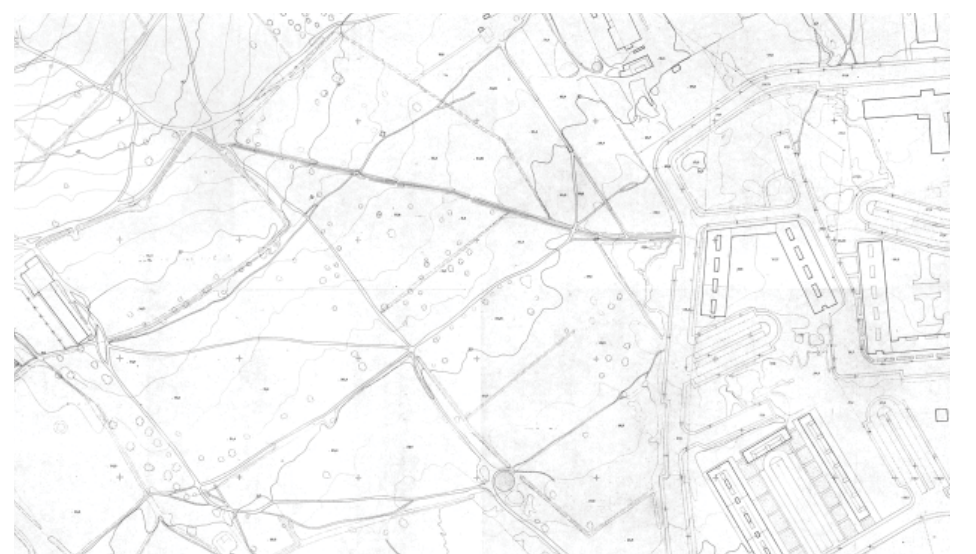

Tarragona. Plano Hoja 21-15 1983_COACT_H-21-15

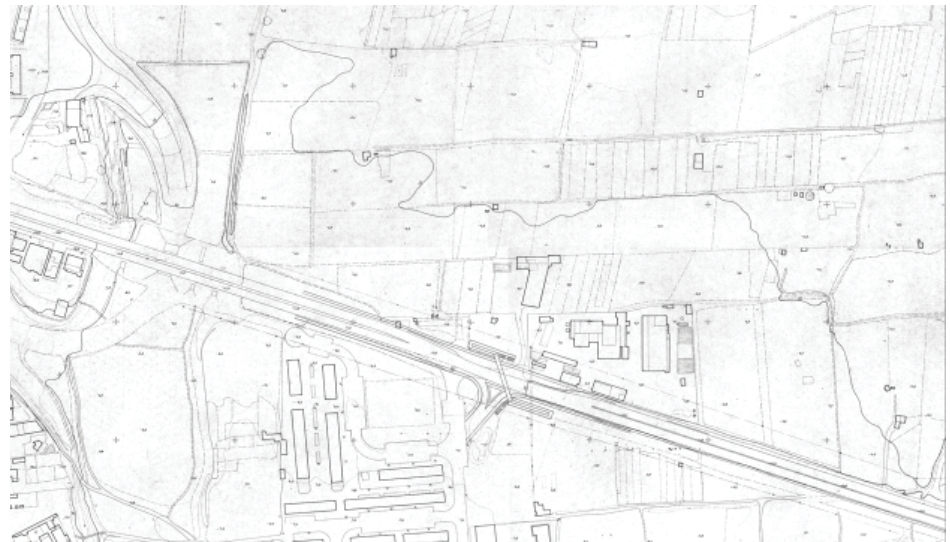

Tarragona. Plano Hoja 21-17 $\quad$ 1983_COÂCT_H-21-17

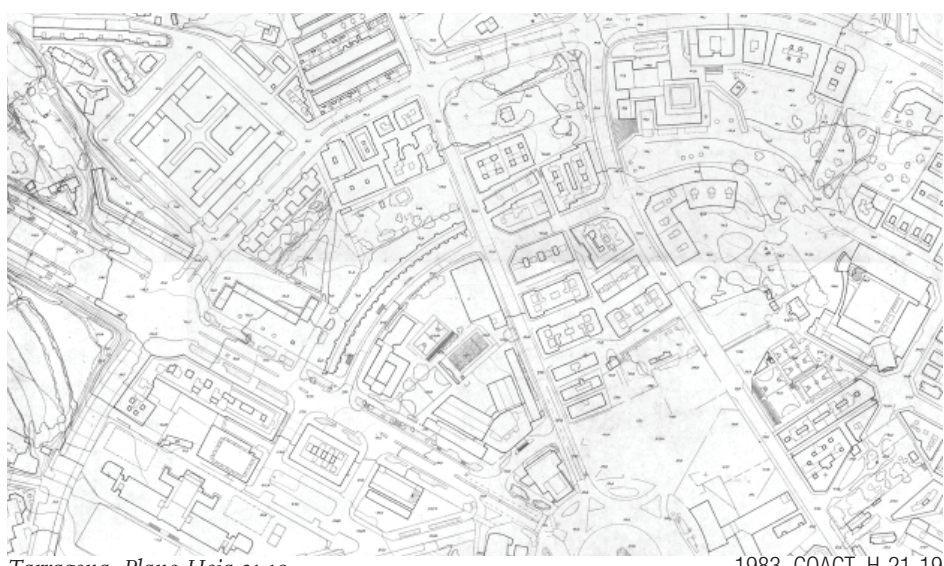

Tarragona. Plano Hoja 21-19

983 COACT H-21-19

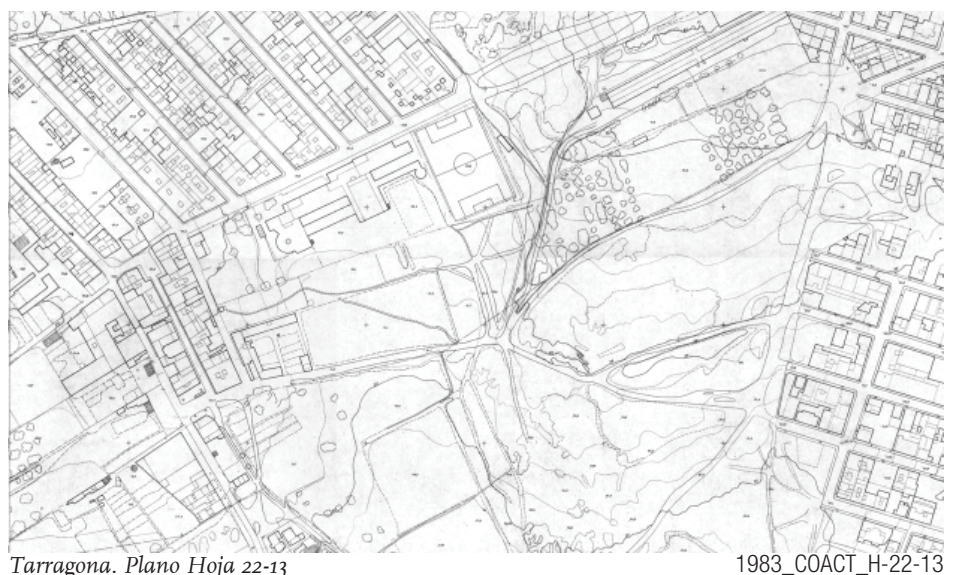

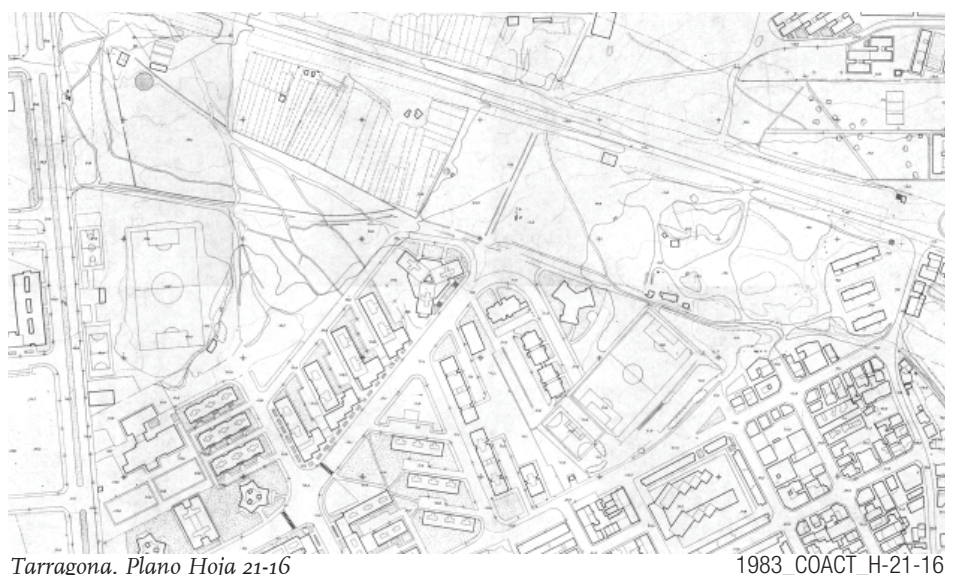

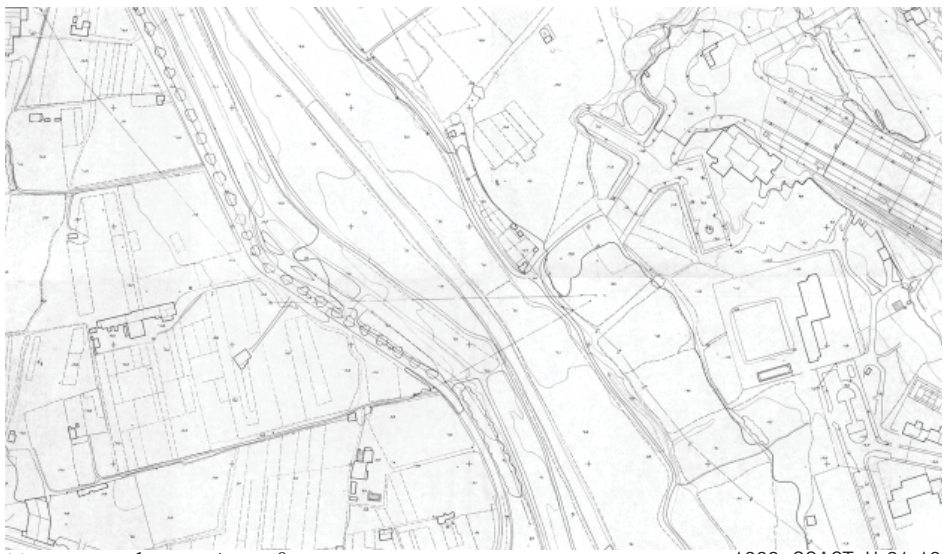

Tarragona. Plano Hoja 21-18

1983_COACT_H-21-18
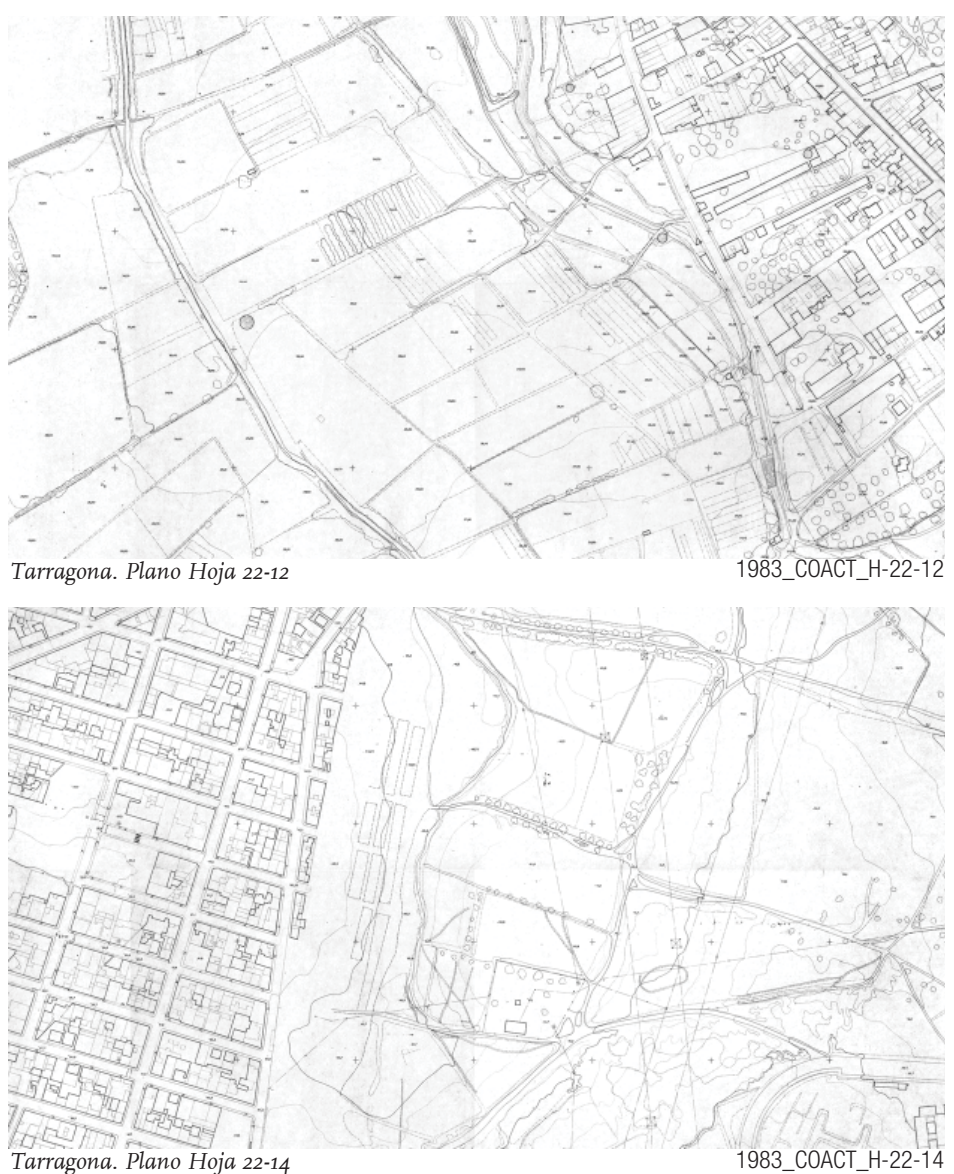


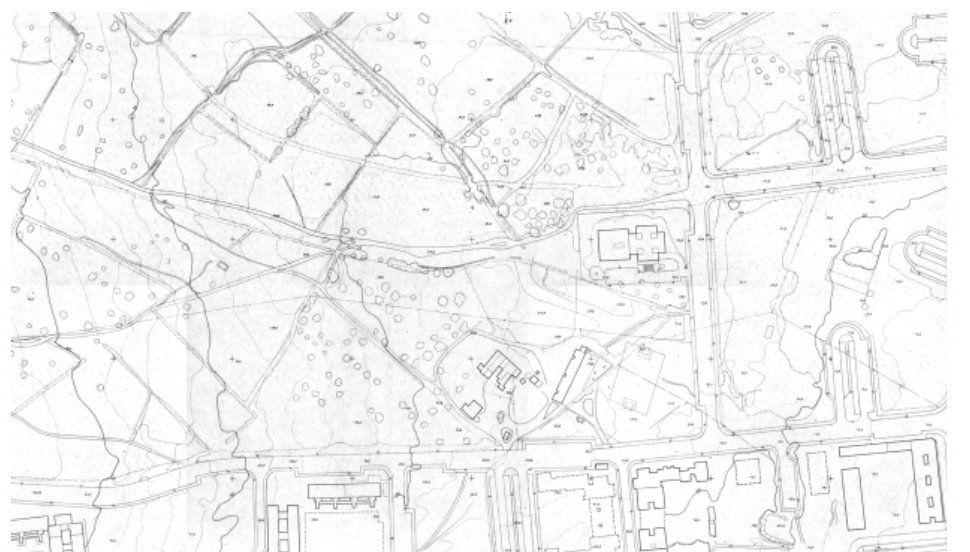

Tarragona. Plano Hoja 22-15

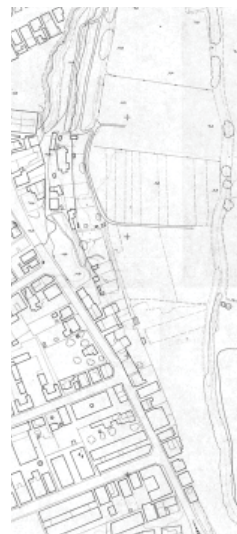

Tarragona. Plano Hoja 22-17

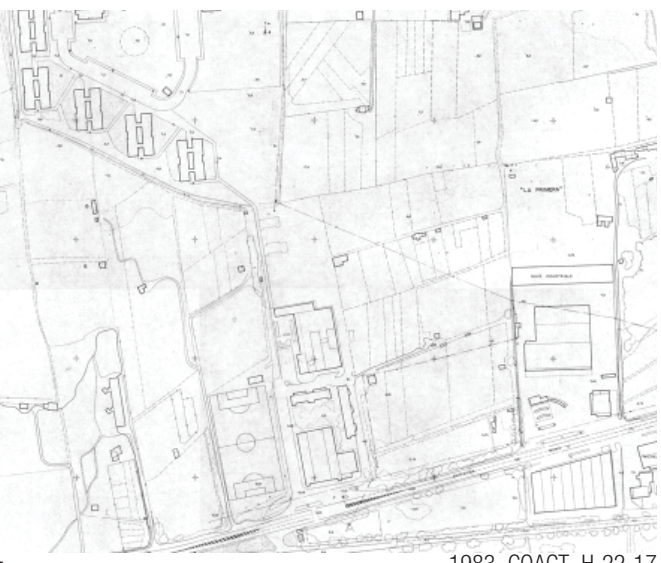

1983 COACT H-22-17

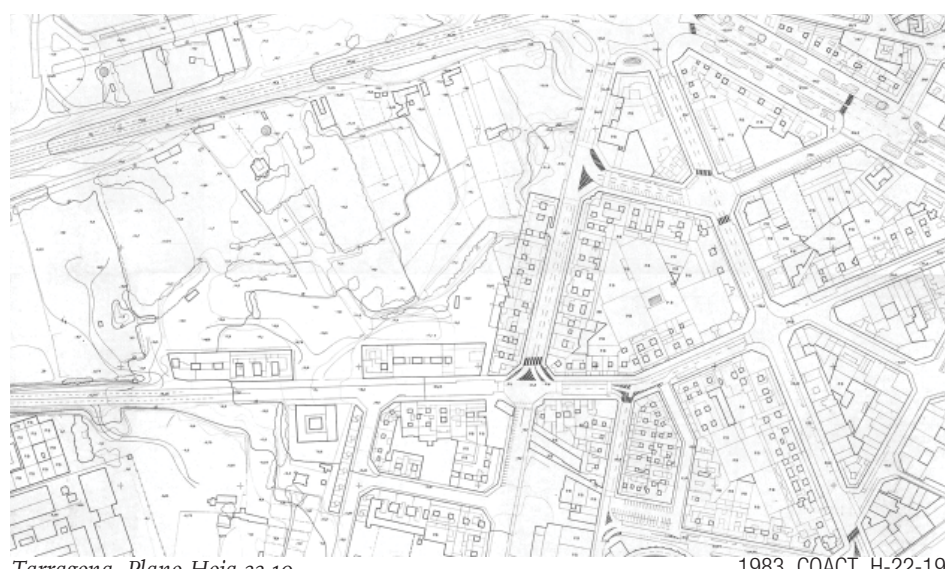

Tarragona. Plano Hoja 22-19

1983 COACT H-22-19

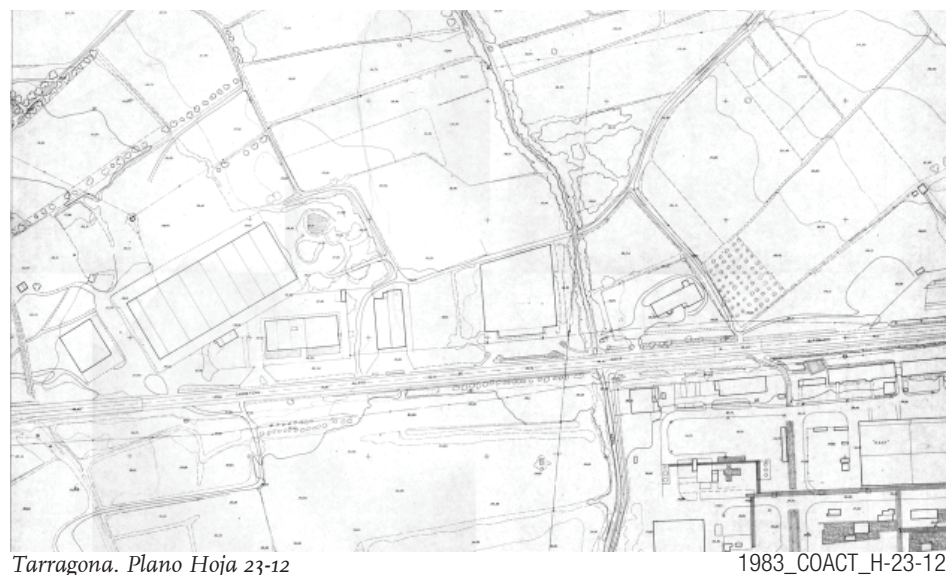

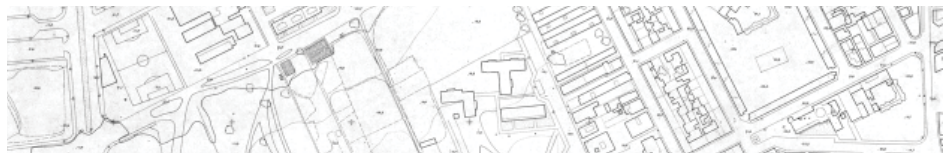

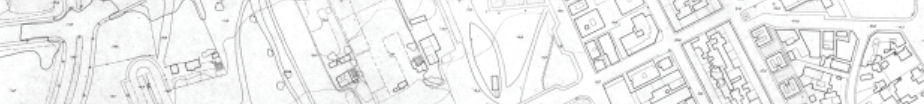
H.

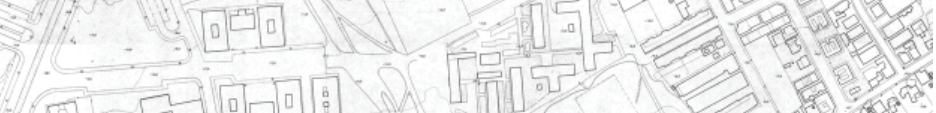

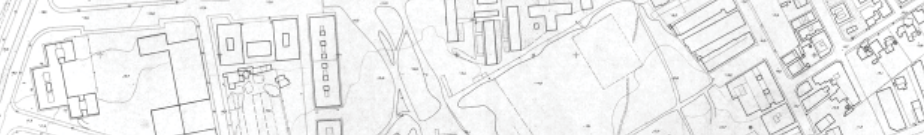
(15) Tarragona. Plano Hoja 22-16 $\quad$ 1983_COACT H-22-16

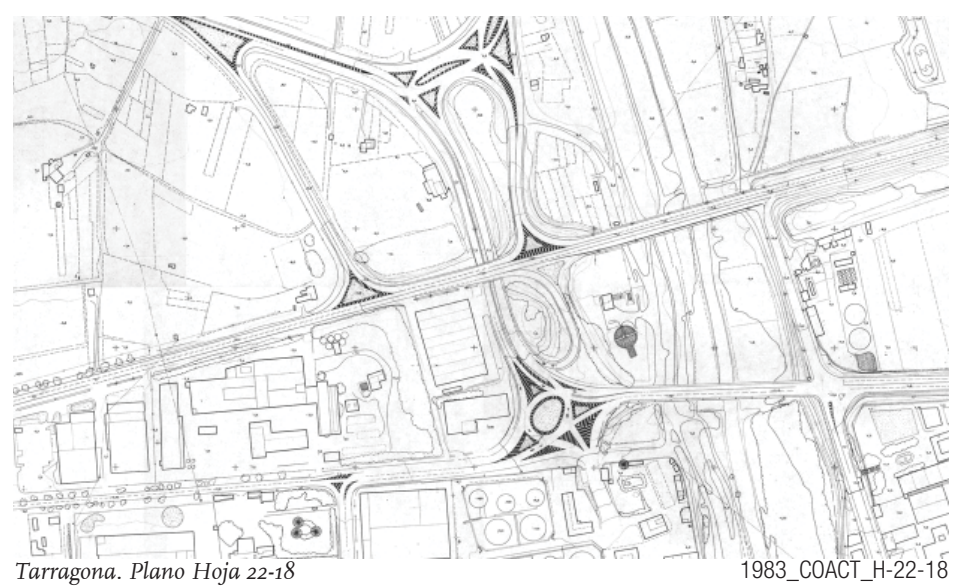

Tarragona. Plano Hoja 22-18
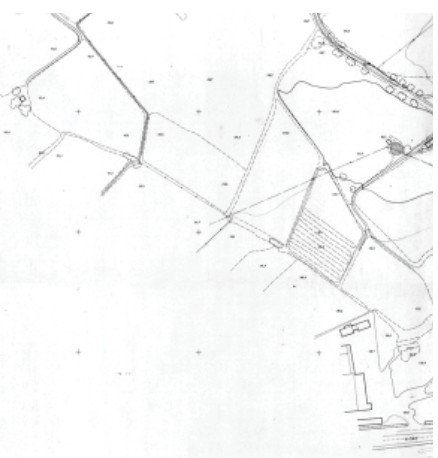

an:

1983_COACT_H-23-11

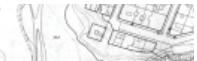

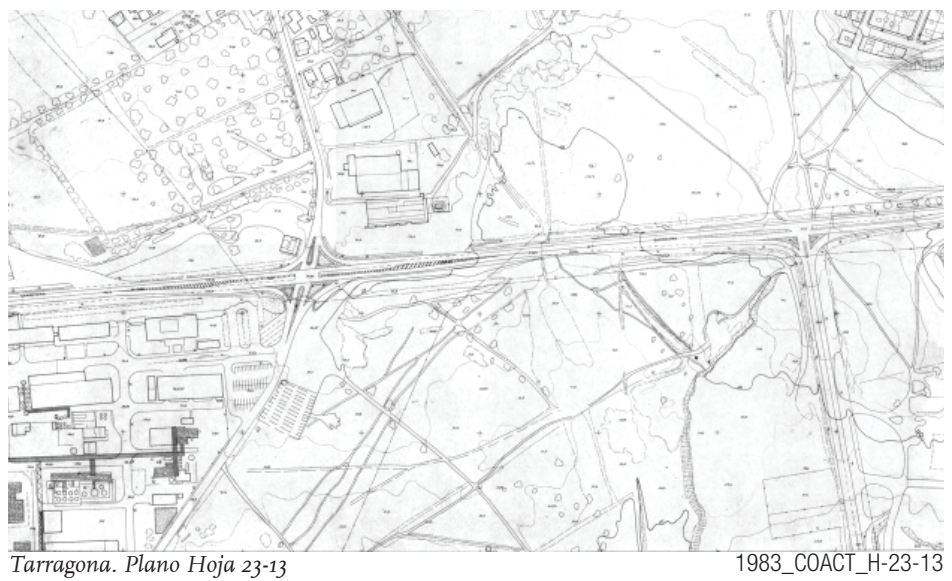




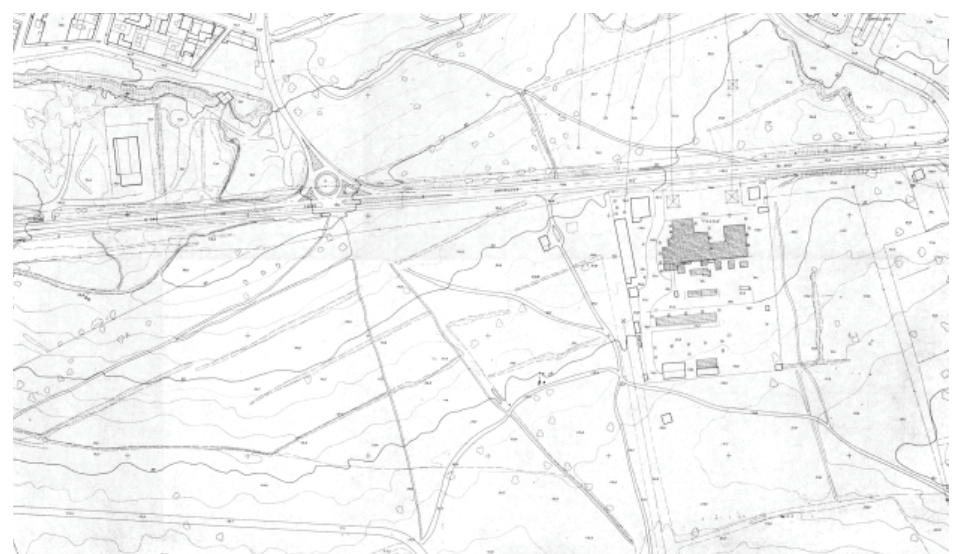

Tarragona. Plano Hoja 23-14

1983 COACT H-23-14

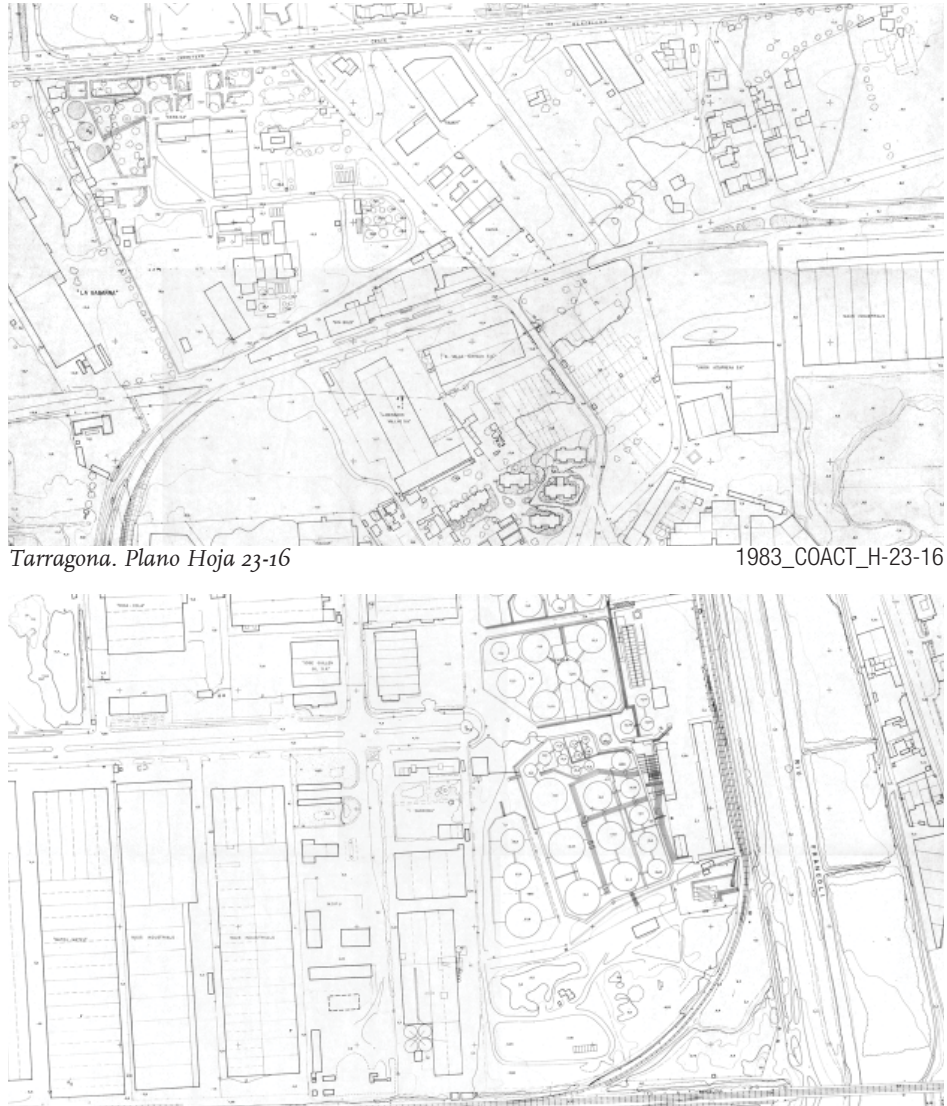

Tarragona. Plano

ano Hoja 23-18

1983 COACT H-23-18

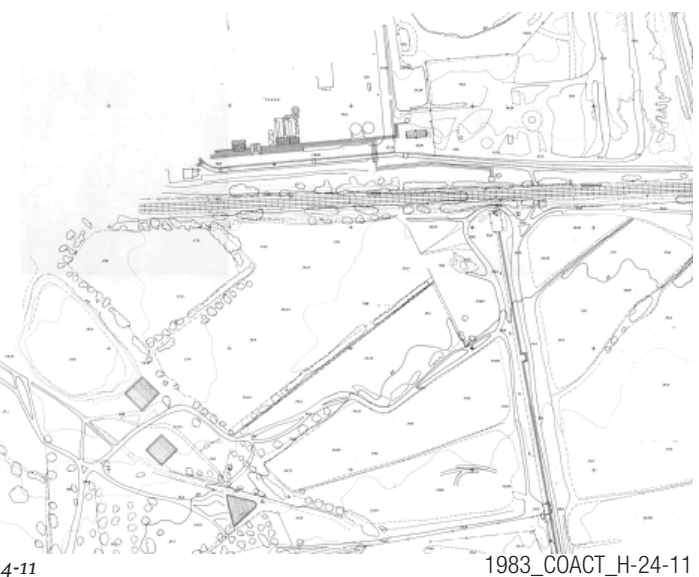

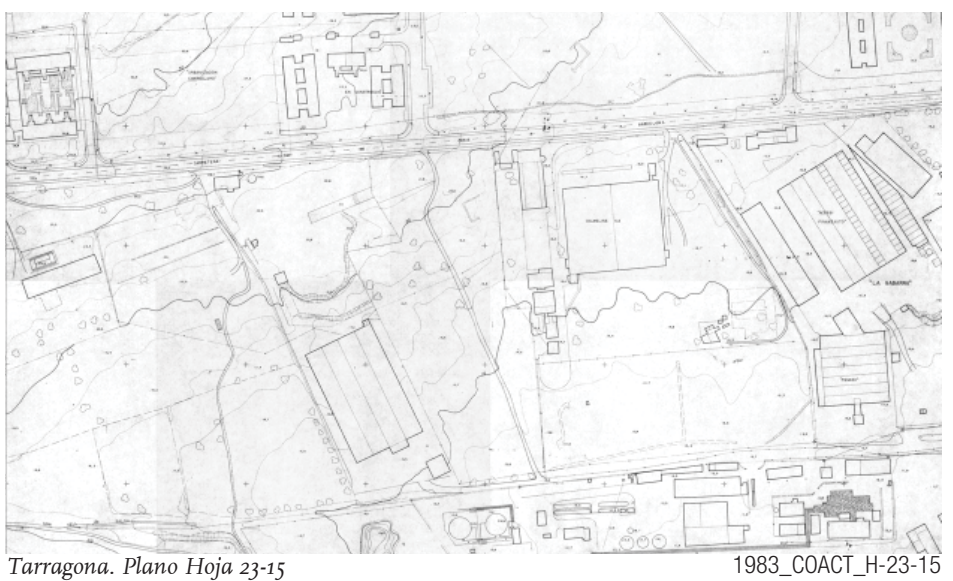
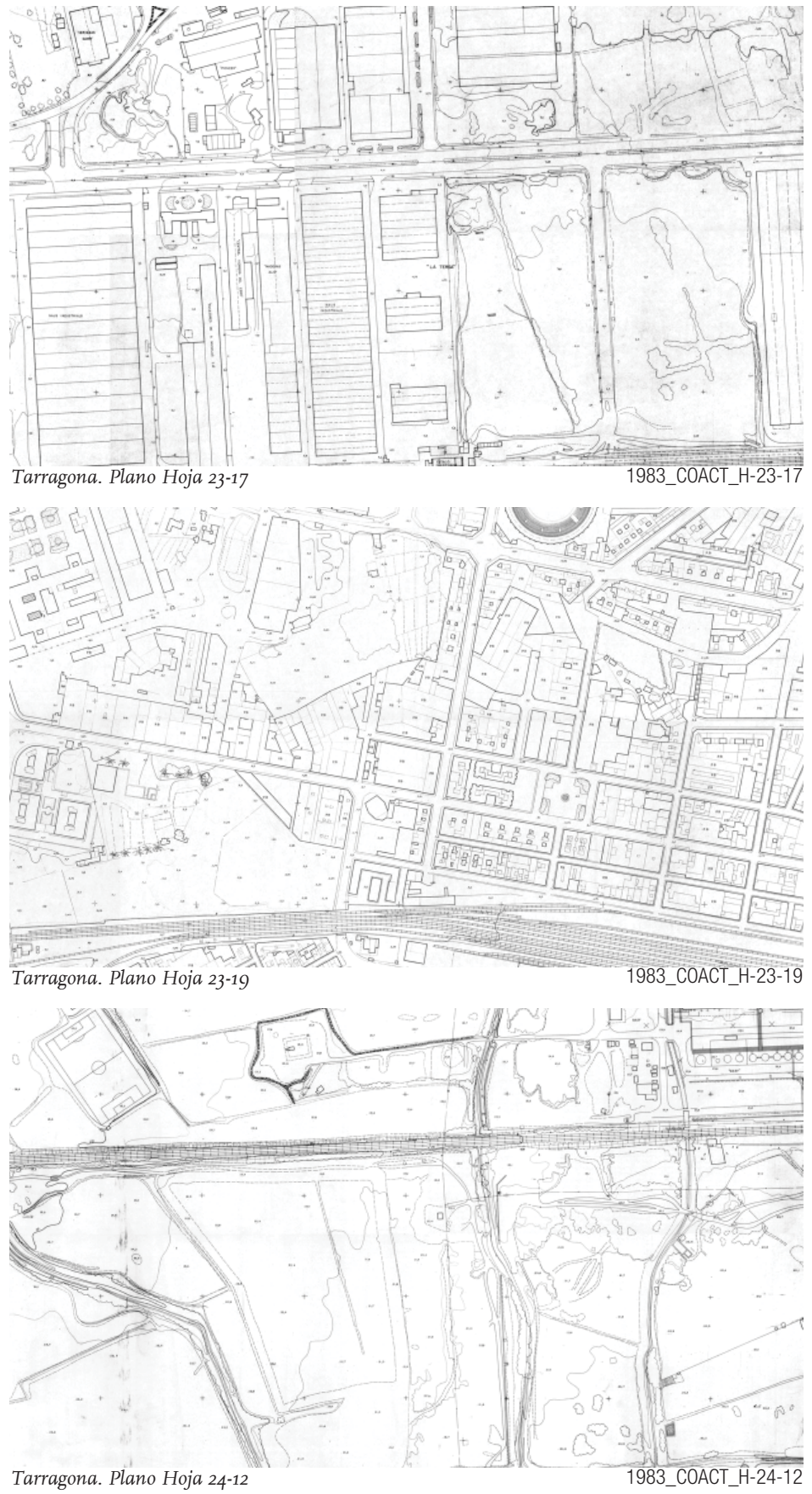
If

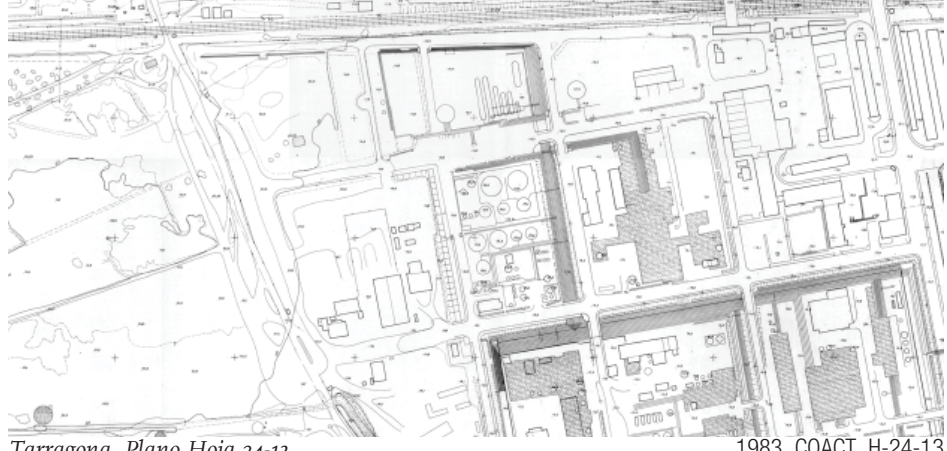

Tarragona. Plano Hoja 24-13

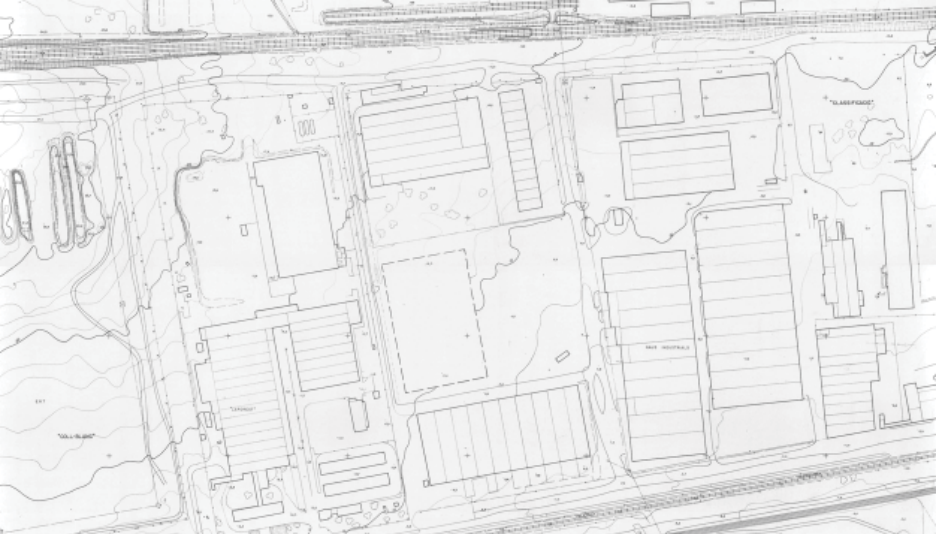

Tarragona. Plano Hoja 24-15 1983_COACT_H-24-15

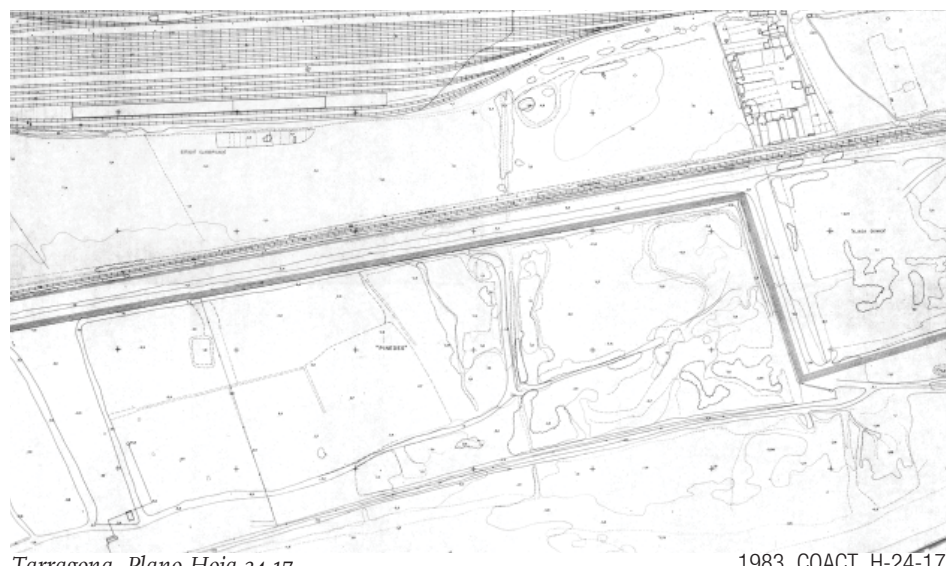

Tarragona. Plano Hoja 24-17

1983 COACT H-24-17

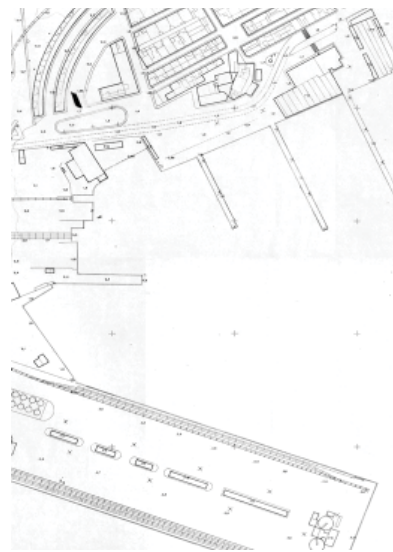

Tarragona. Plano Hoja 24-19

-

1983_COACT_H-24-19

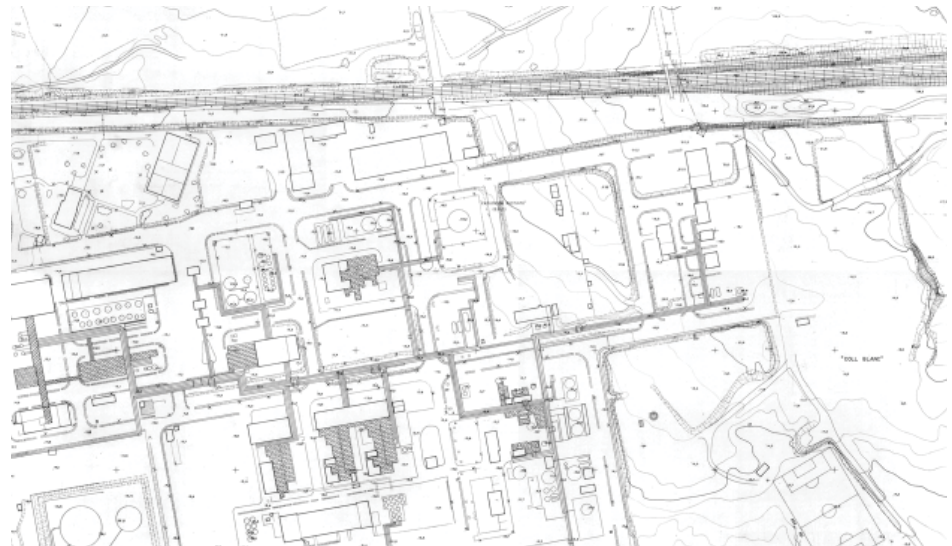

Tarragona. Plano Hoja 24-14

1983_COACT_H-24-14
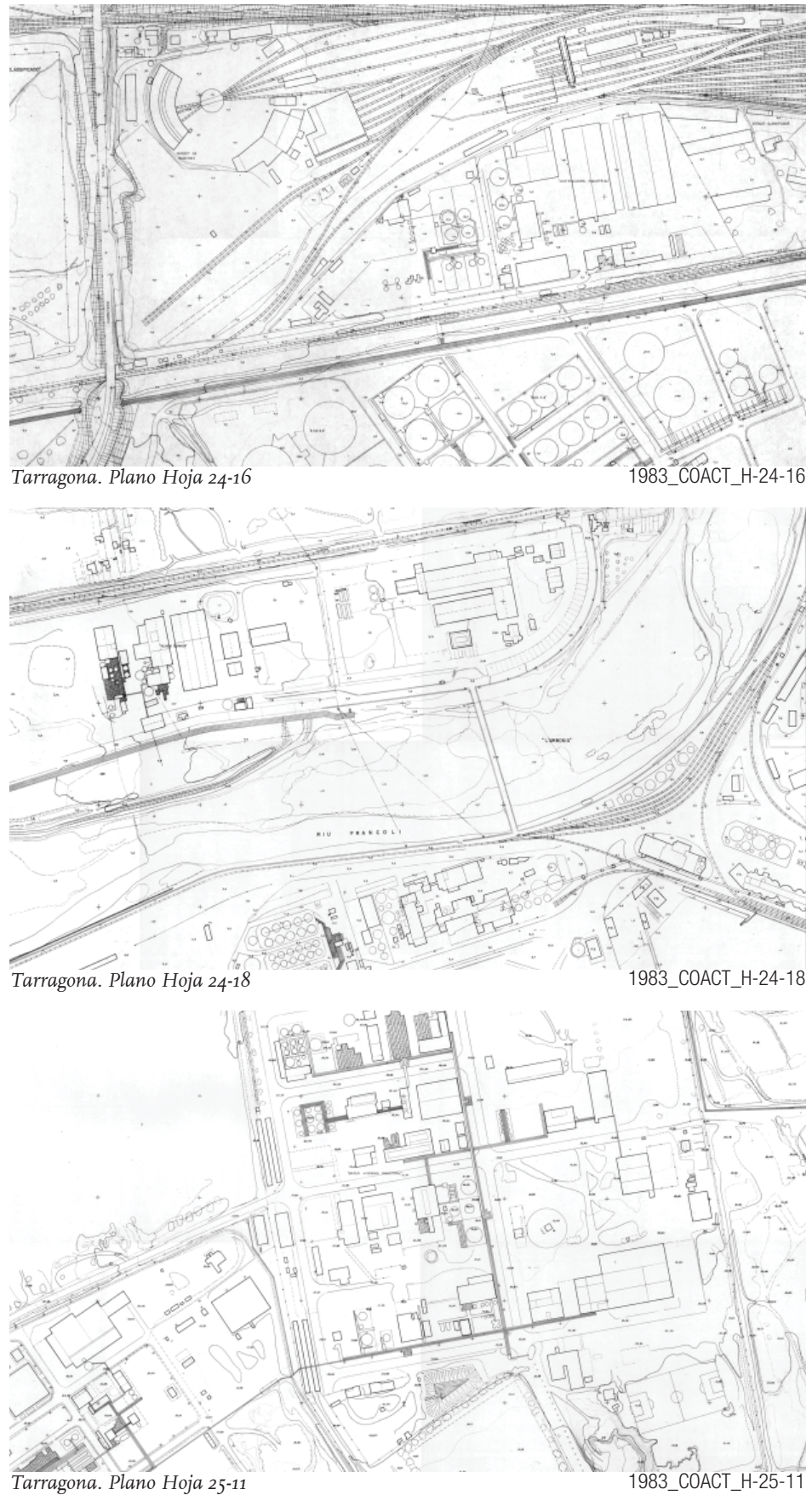


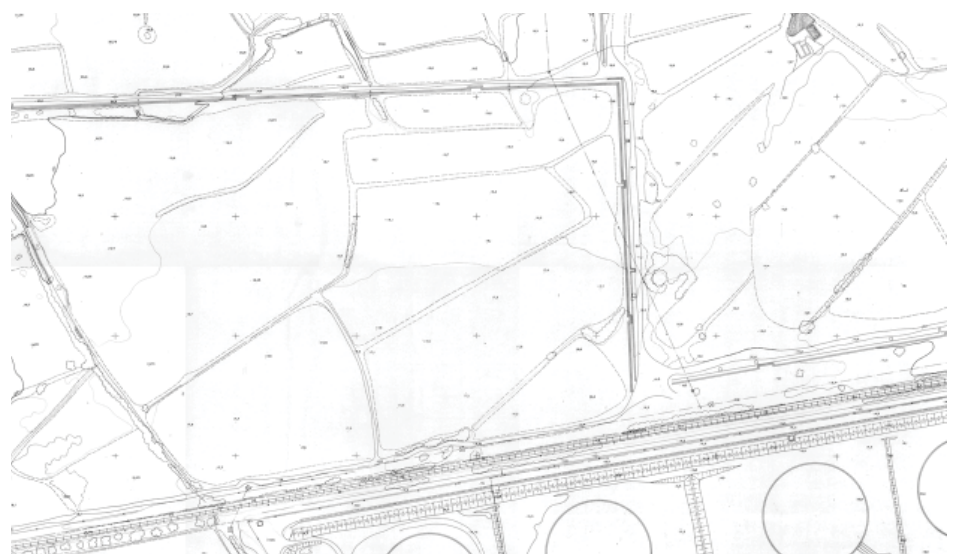

Tarragona. Plano Hoja 25-12

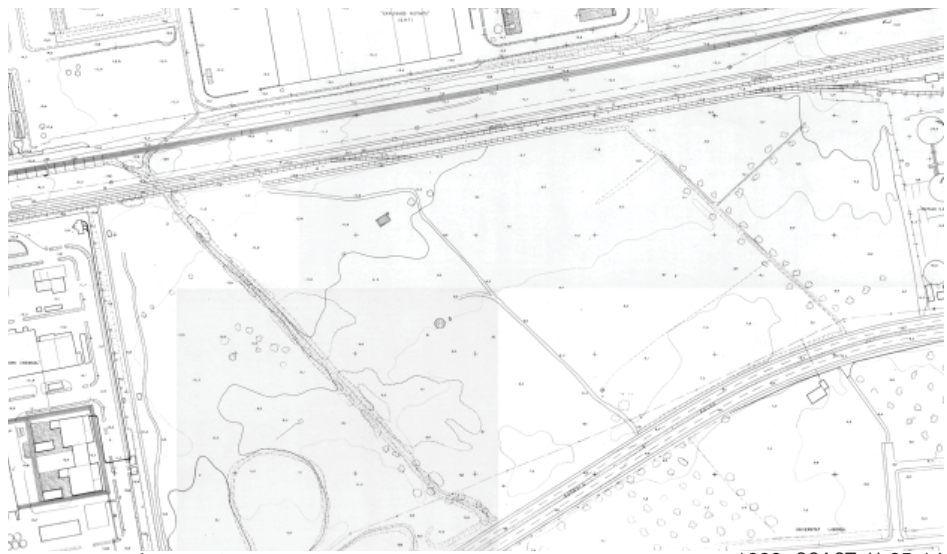

Tarragona. Plano Hoja 25-14

1983_COACT_H-25-14

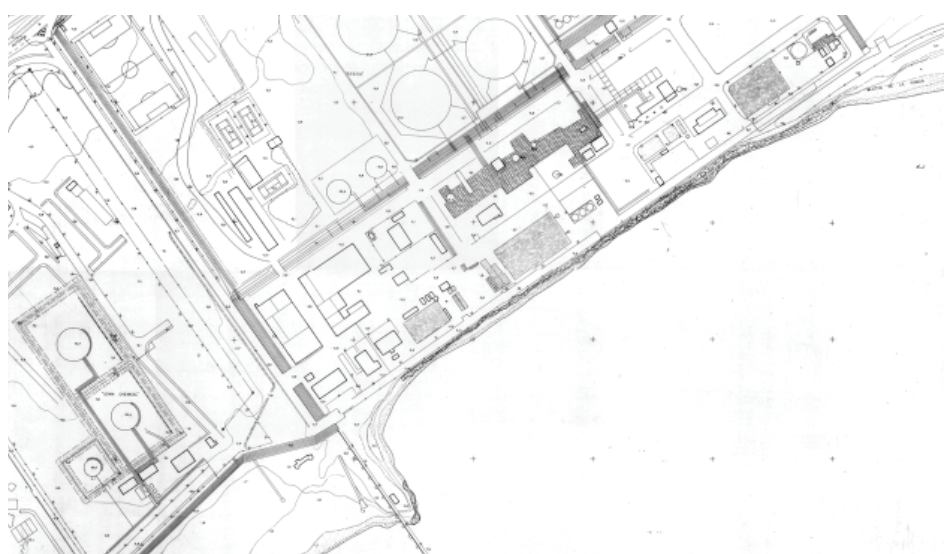

Tarragona. Plano Hoja 25-16

1983_COACT_H-25-16

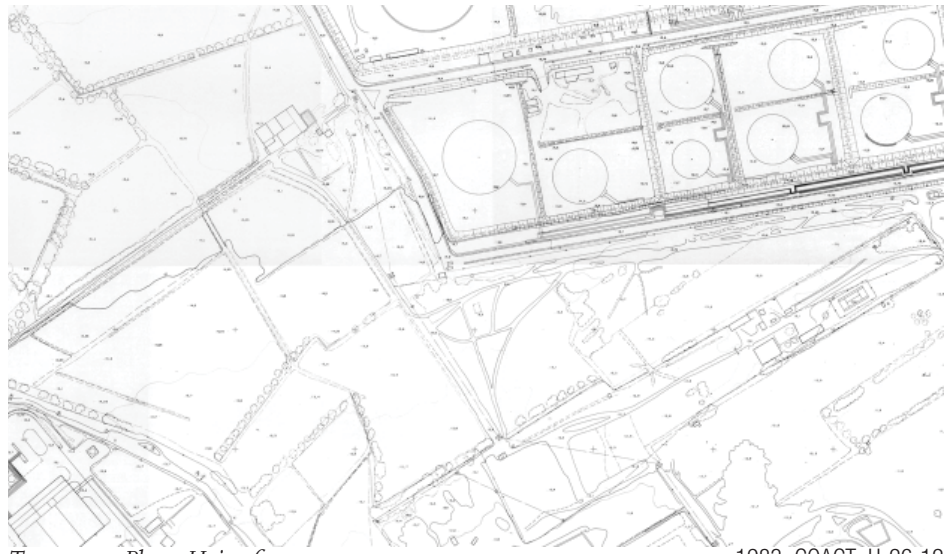

Tarragona. Plano Hoja 26-12

1983_COACT_H-26-12
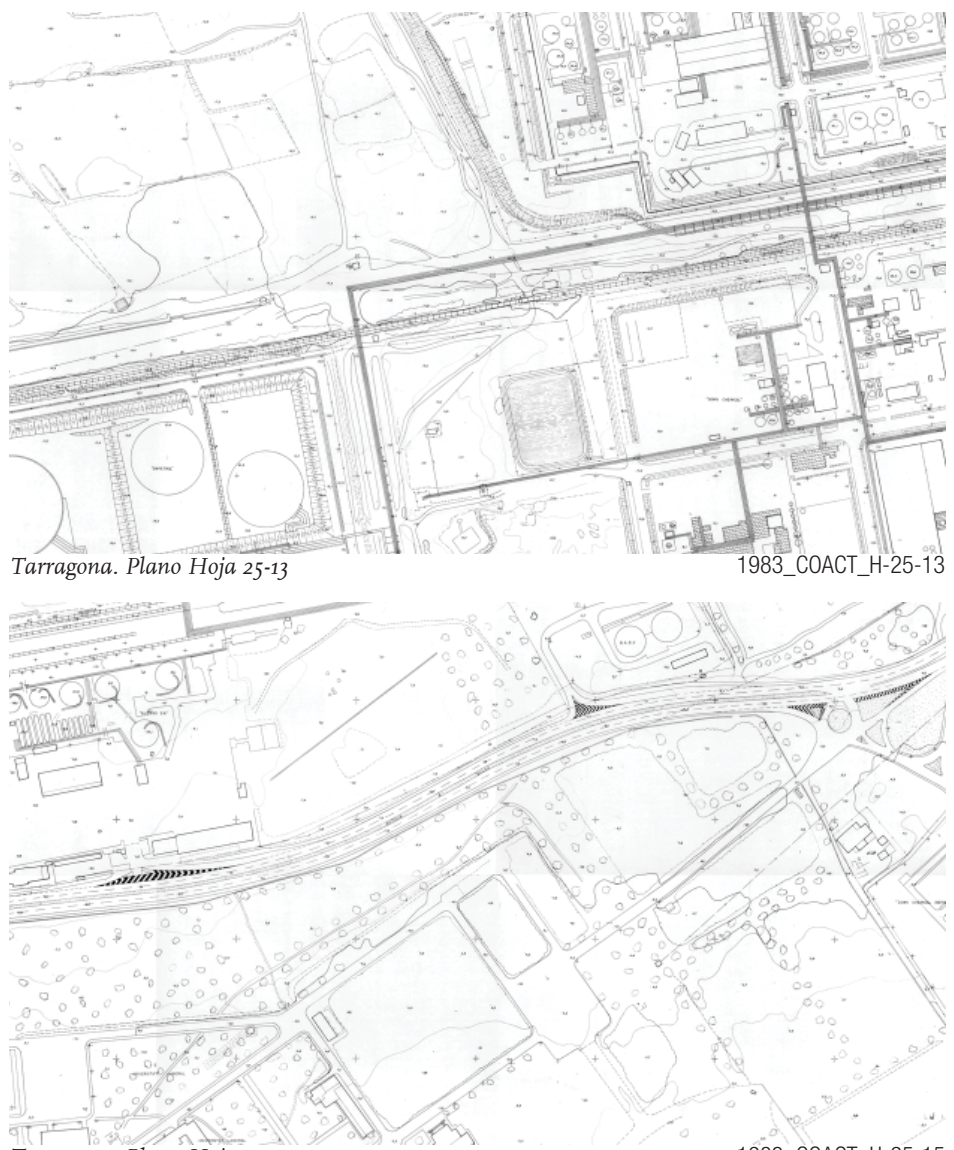

Tarragona. Plano Hoja 25-15

1983_COACT_H-25-15
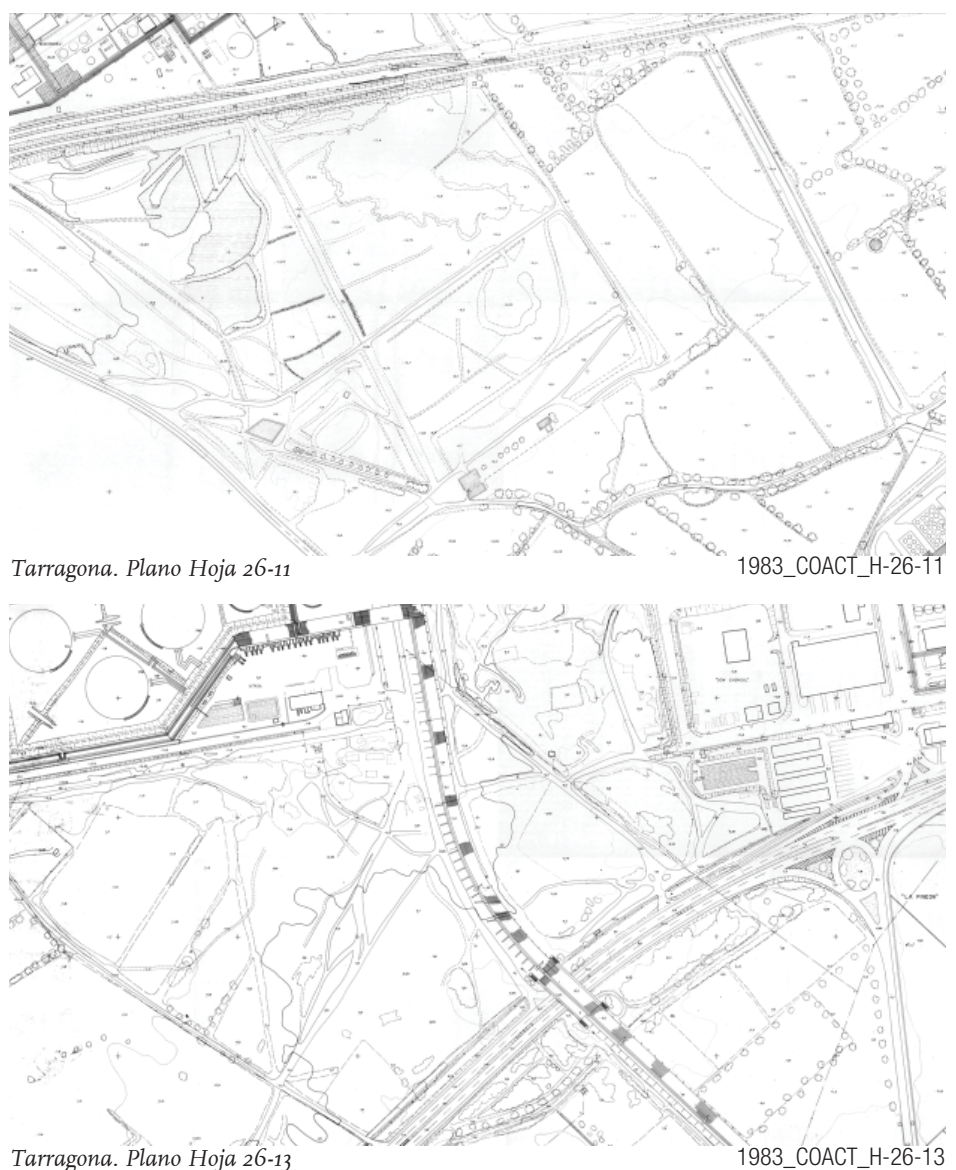


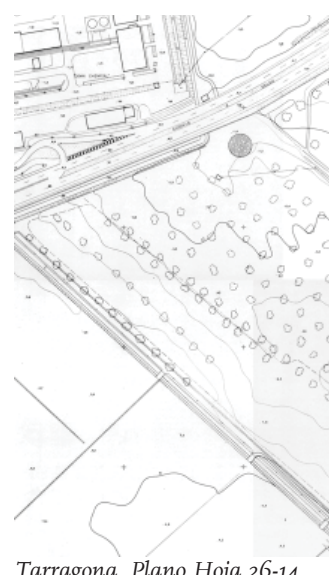

Tarragona. Plano Hoja 26-14
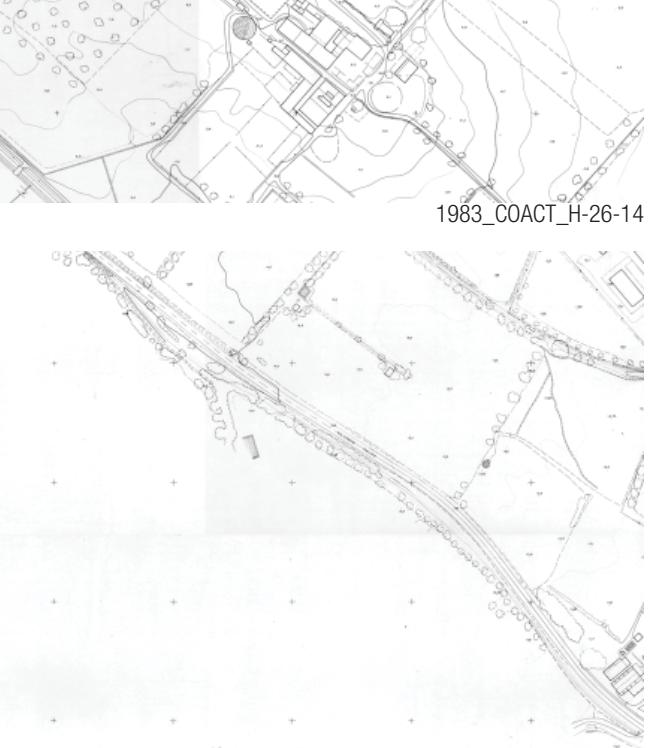

Tarragona. Plano Hoja 27-11

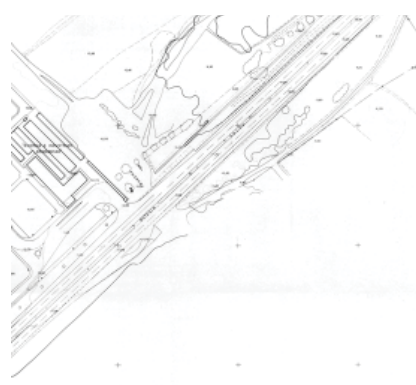

1983_COACT_H-27-11

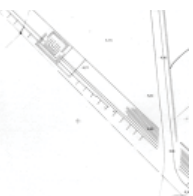

1983_COACT_H-27-13

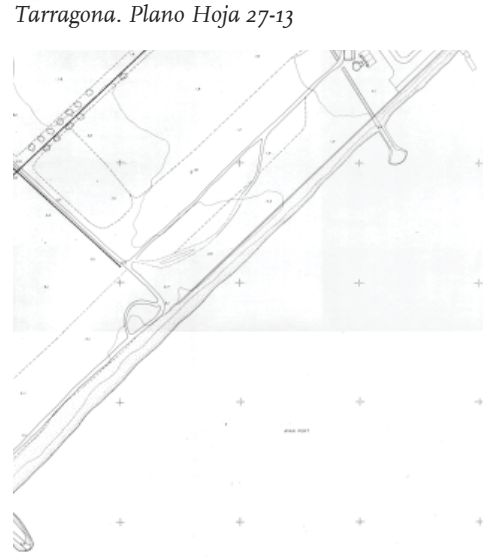

Tarragona. Plano Hoja 27-15

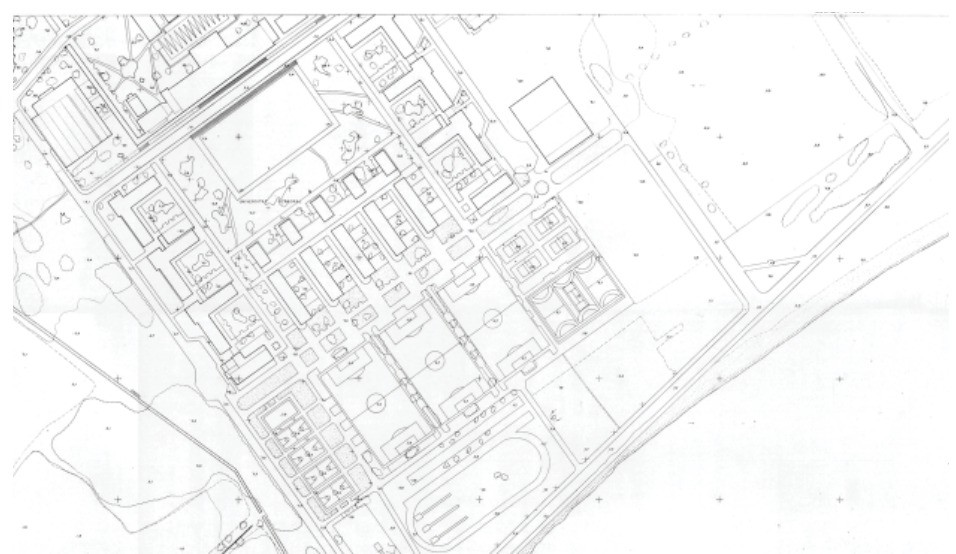

Tarragona. Plano Hoja 26-15

1983_COACT_H-26-15

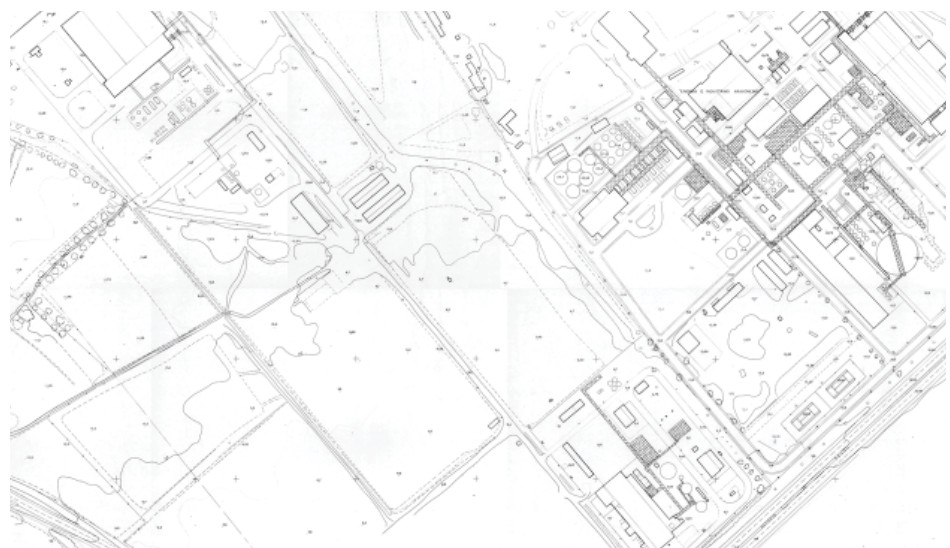

Tarragona. Plano Hoja 27-12

1983_COACT_H-27-12

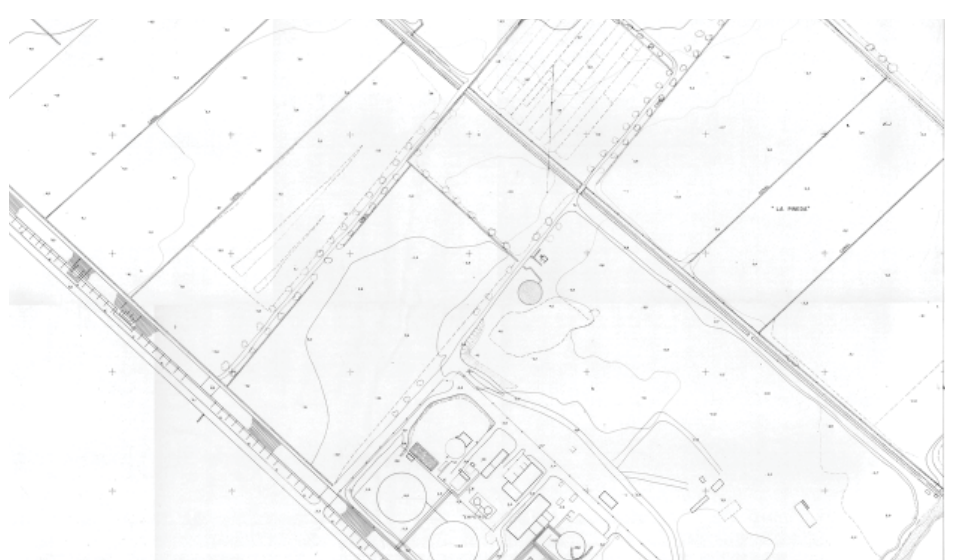

Tarragona. Plano Hoja 27-14

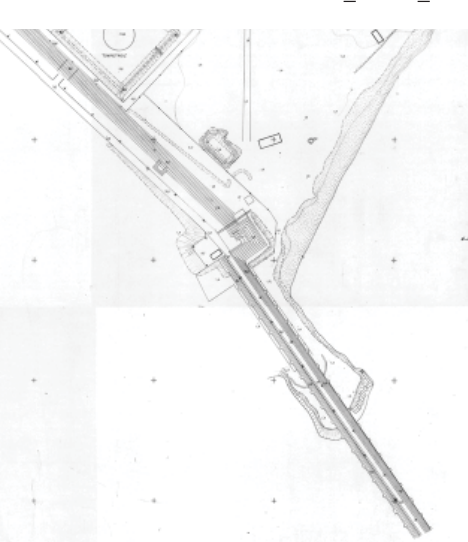

1983_COACT_H-28-14 


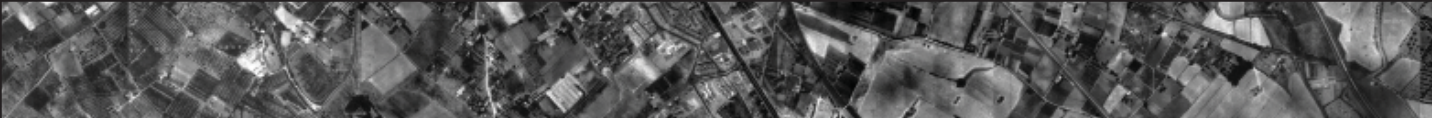

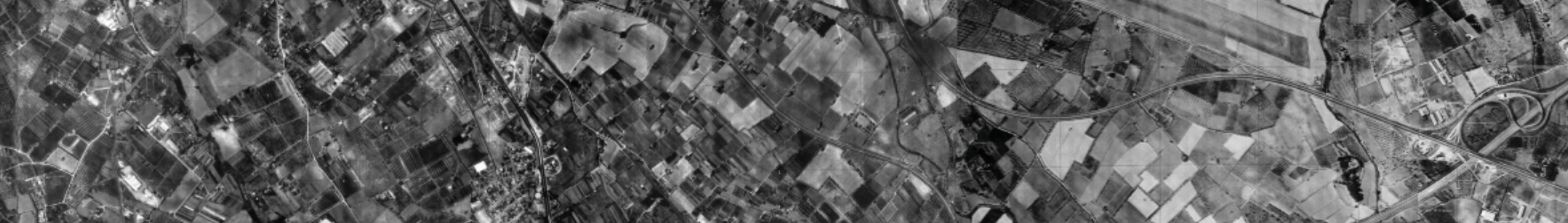

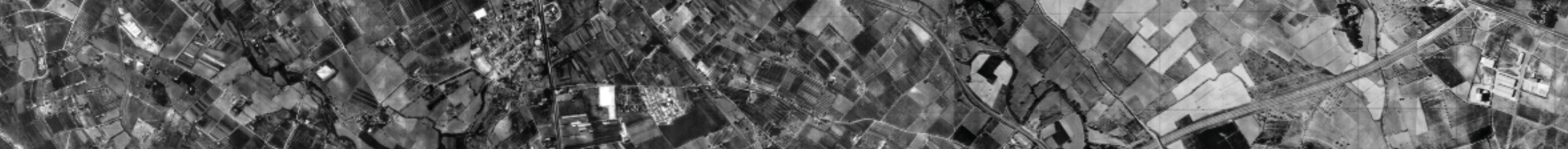

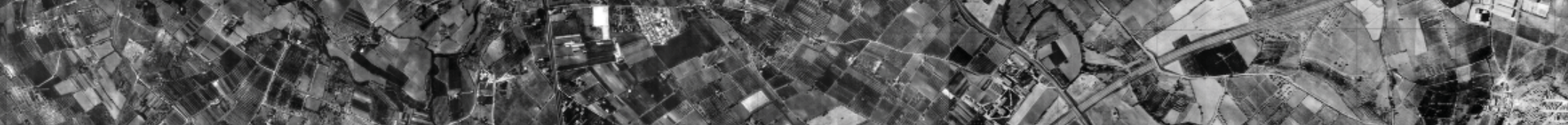

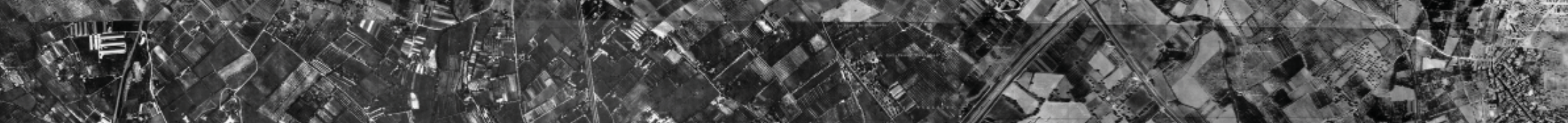

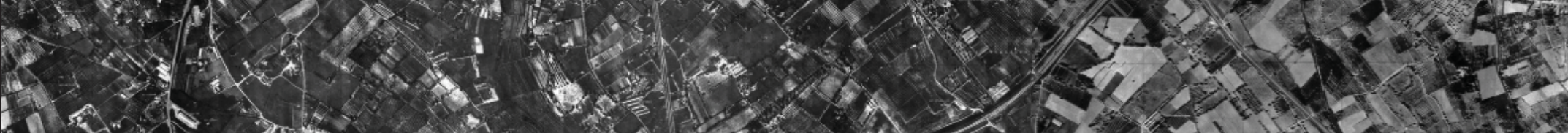

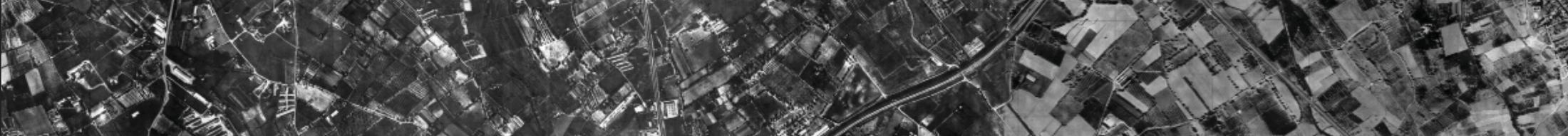

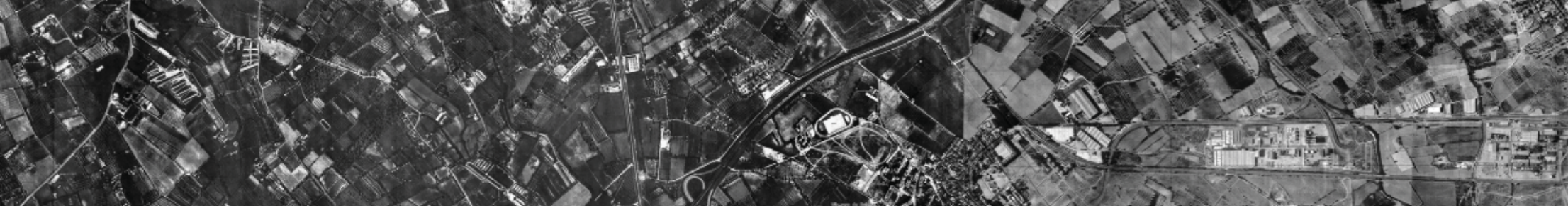

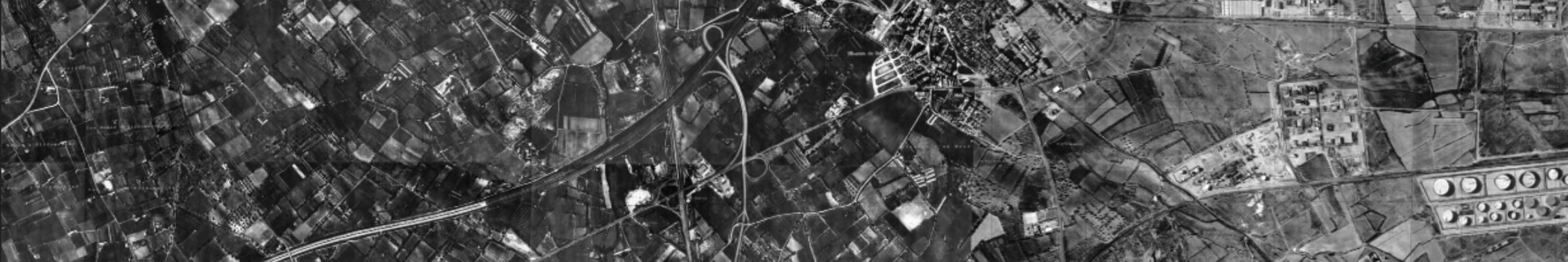

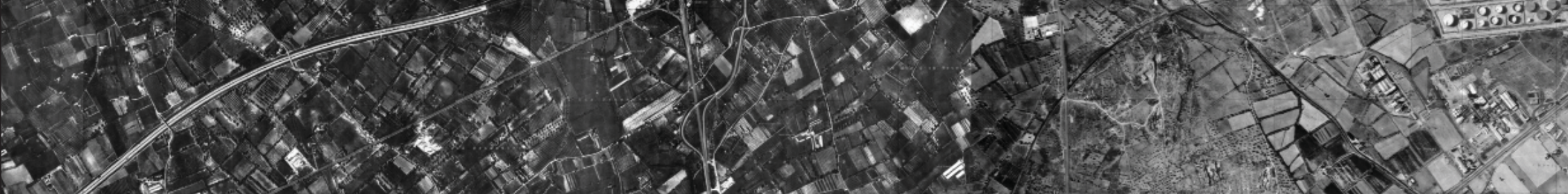

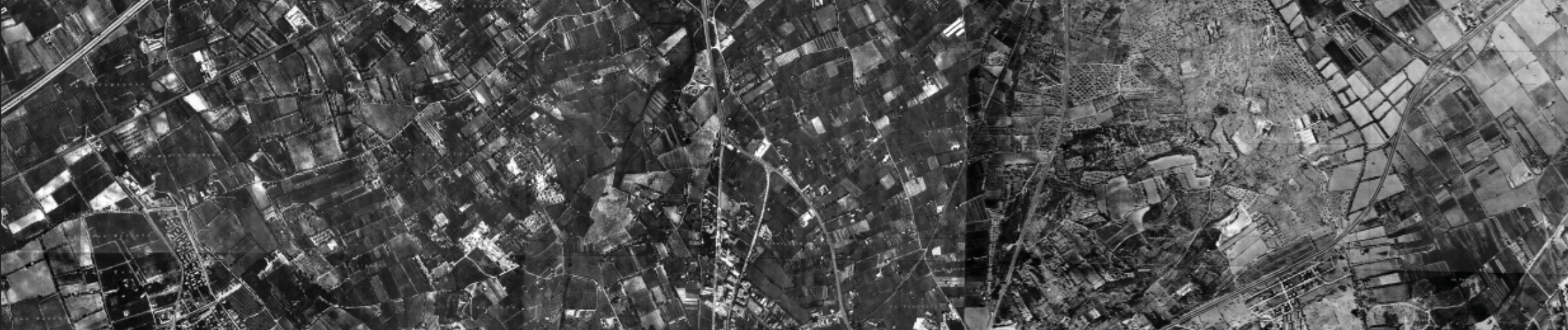
(2) W.1.

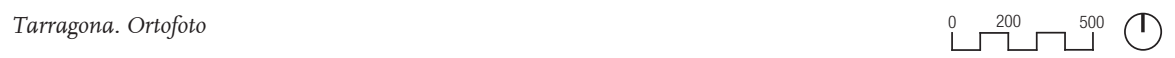

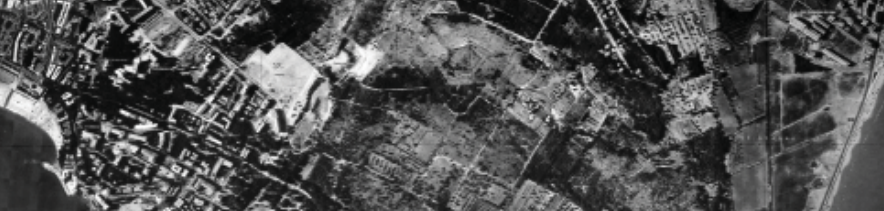

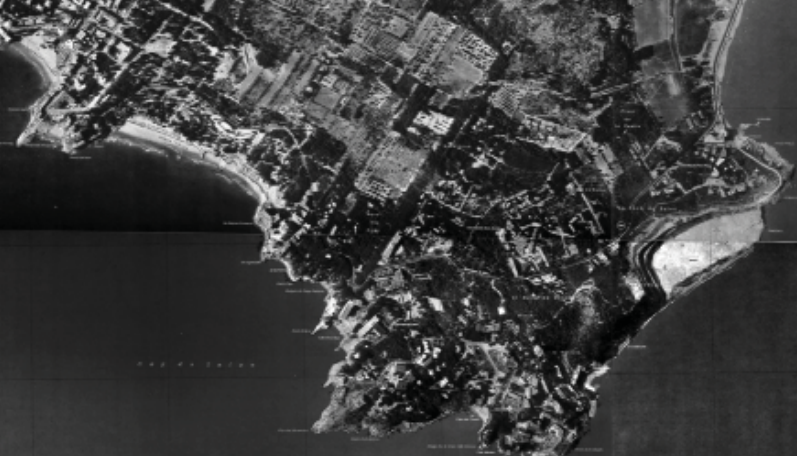




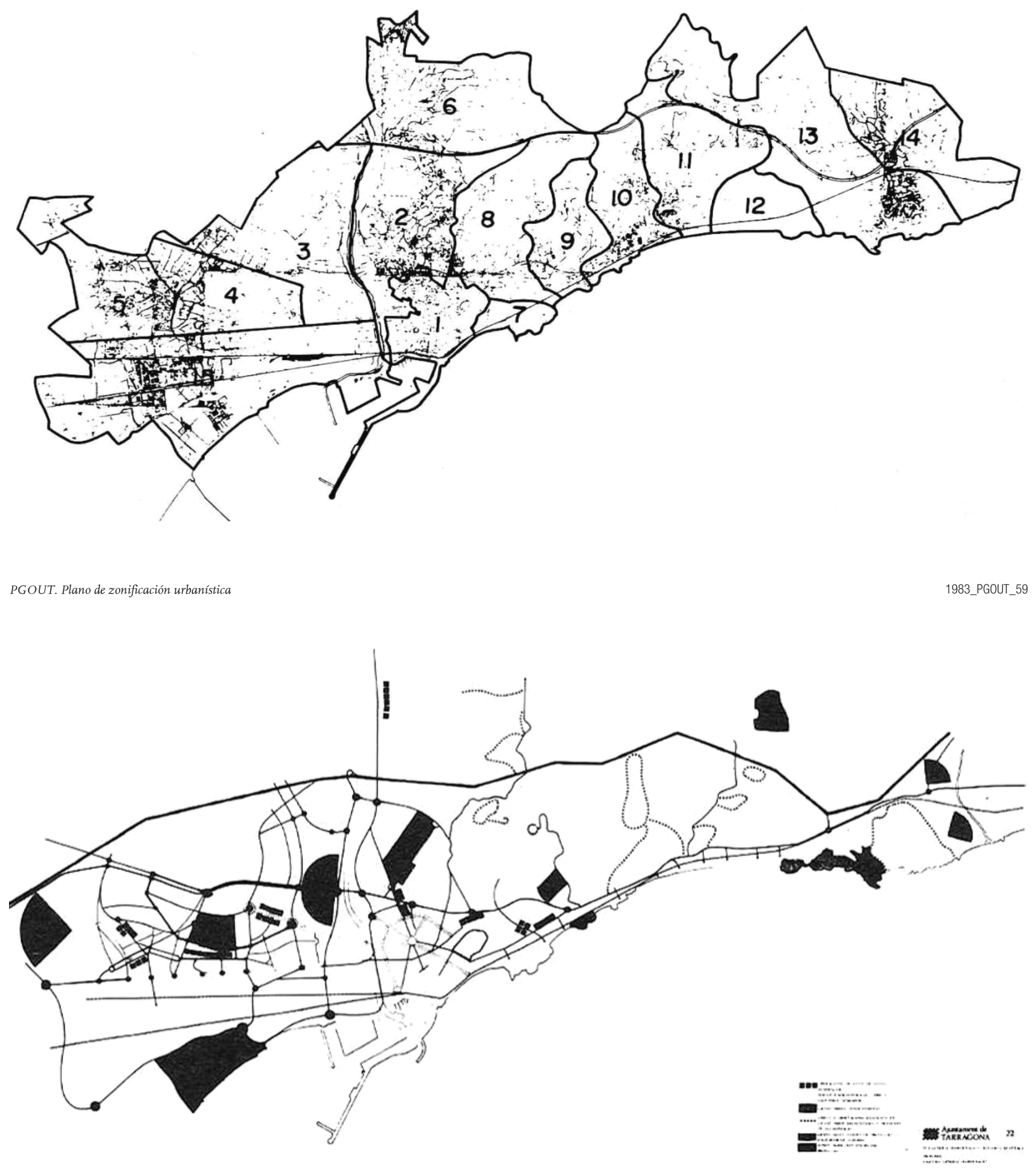

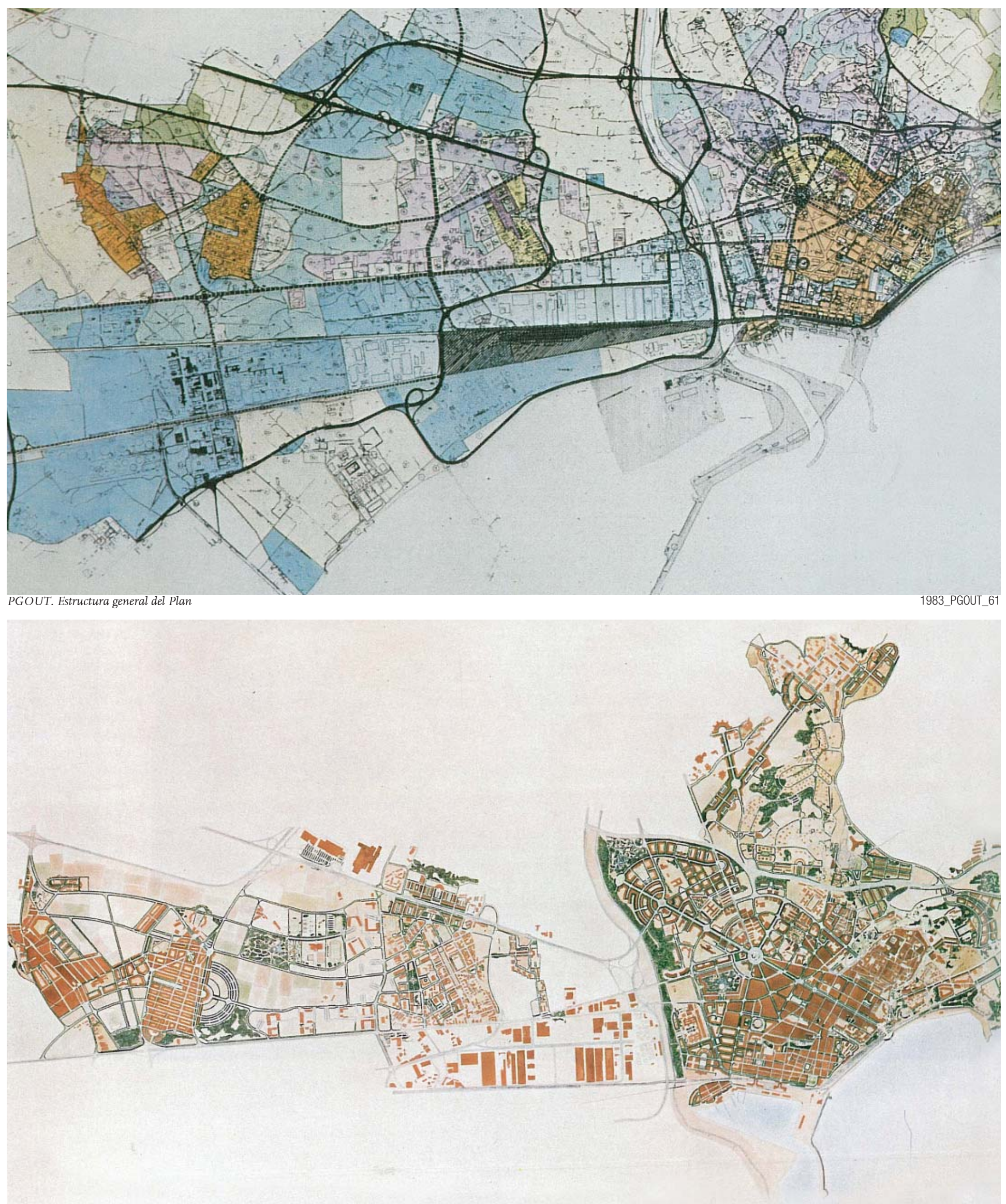

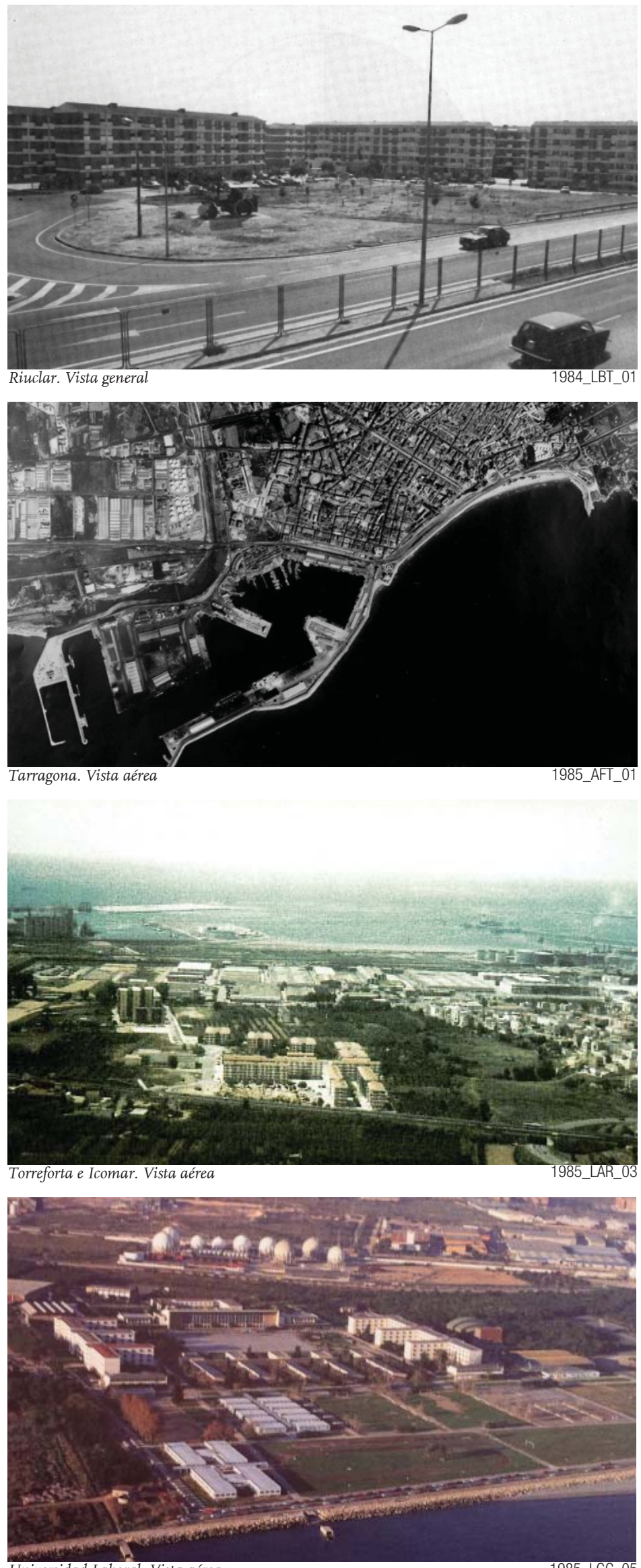

Universidad Laboral. Vista aérea

1985_LGC_05
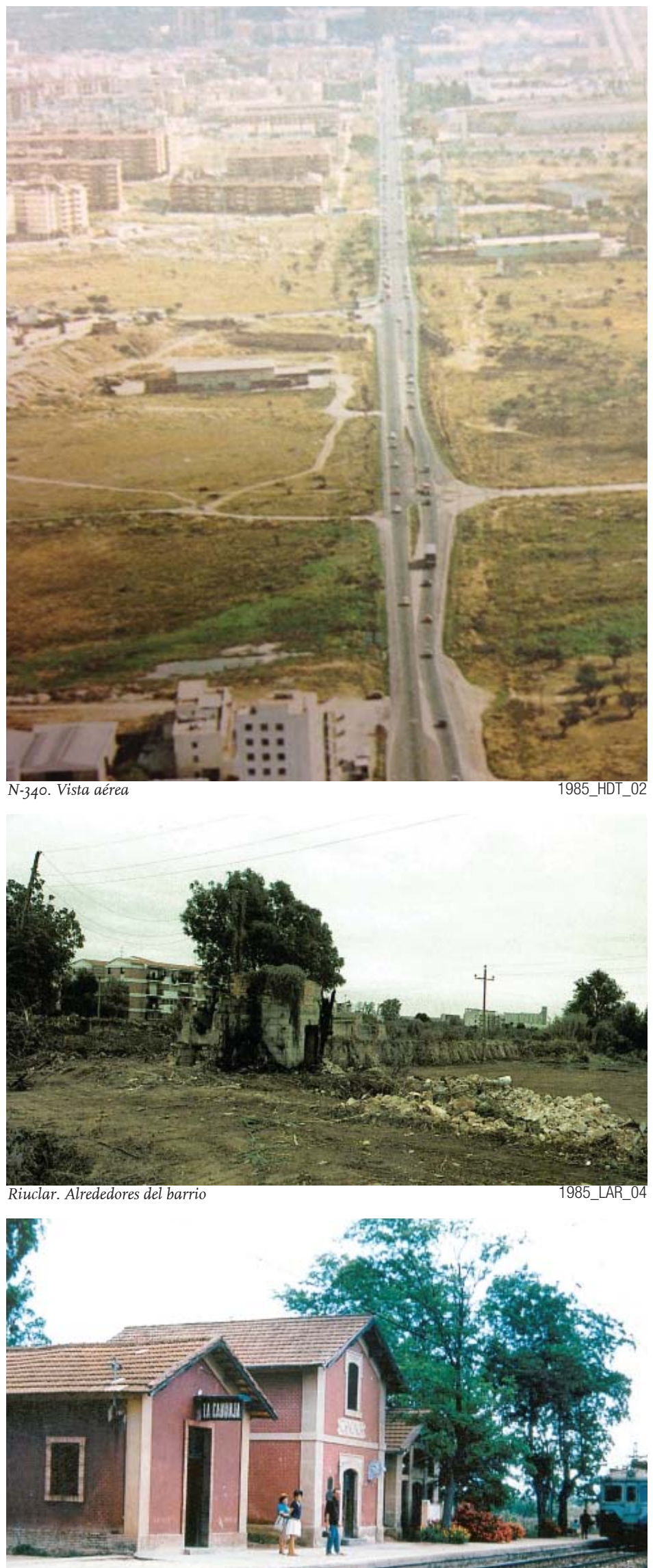

La Canonja. Vista del desaparecido bajador de tren 

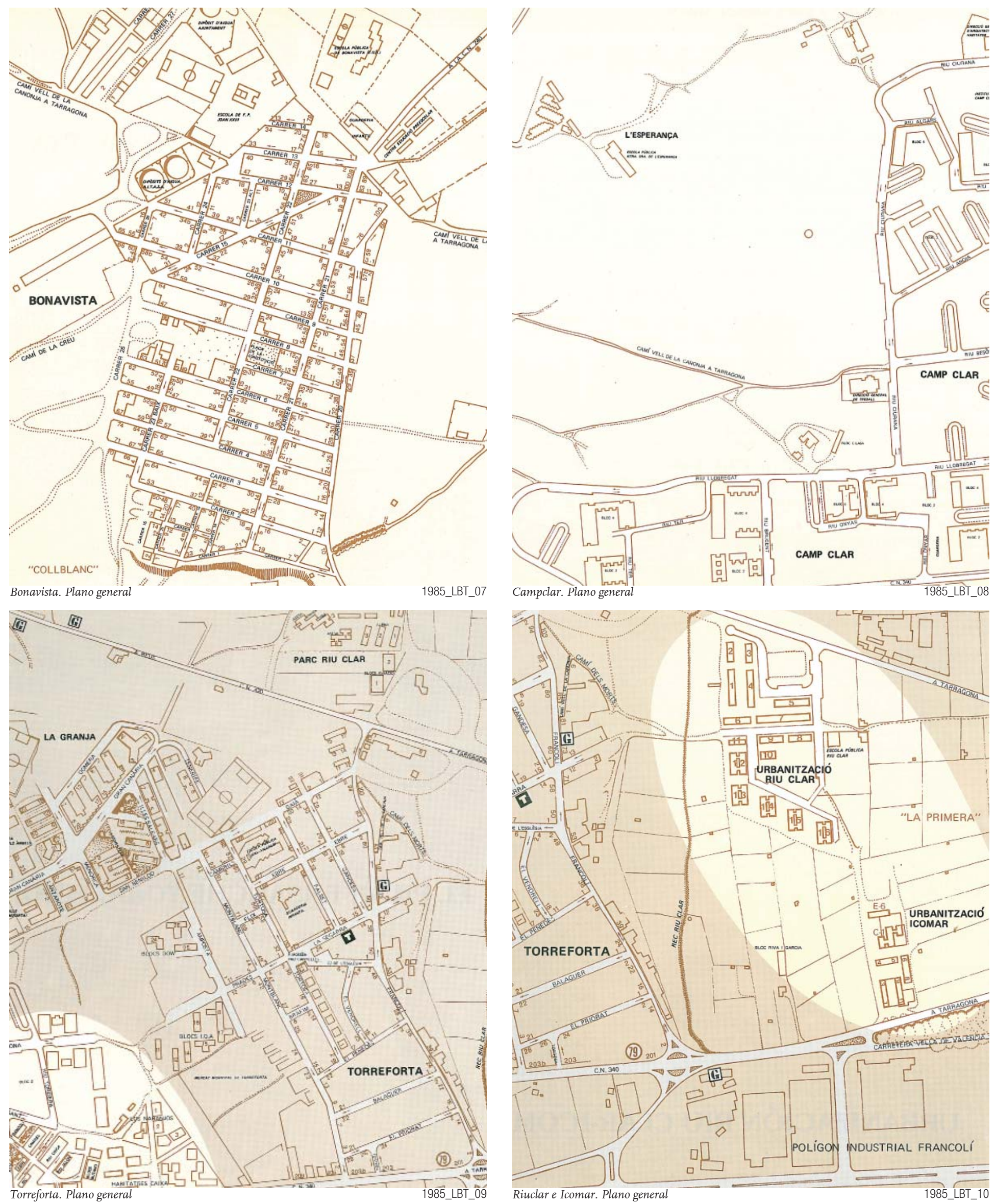


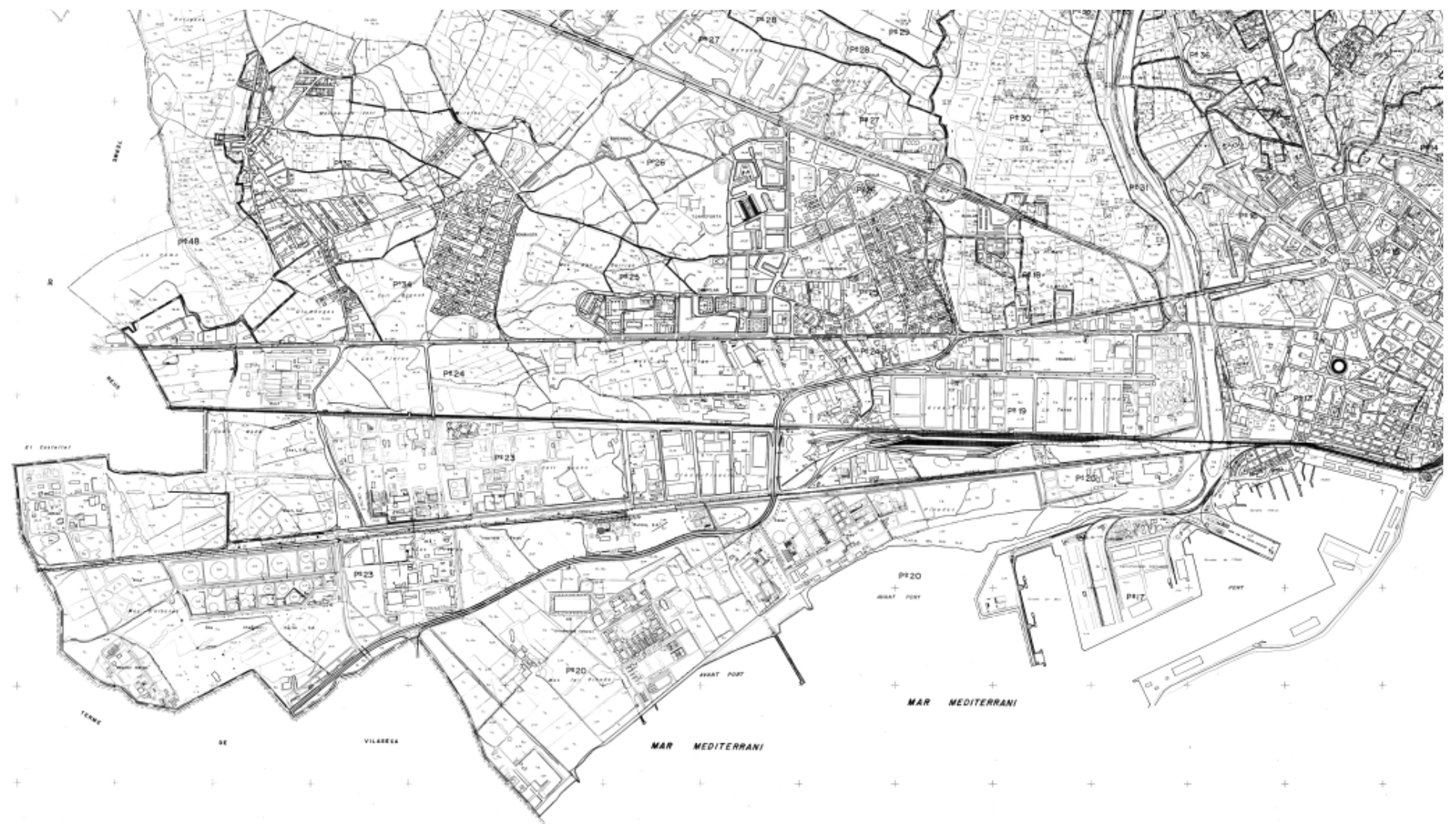

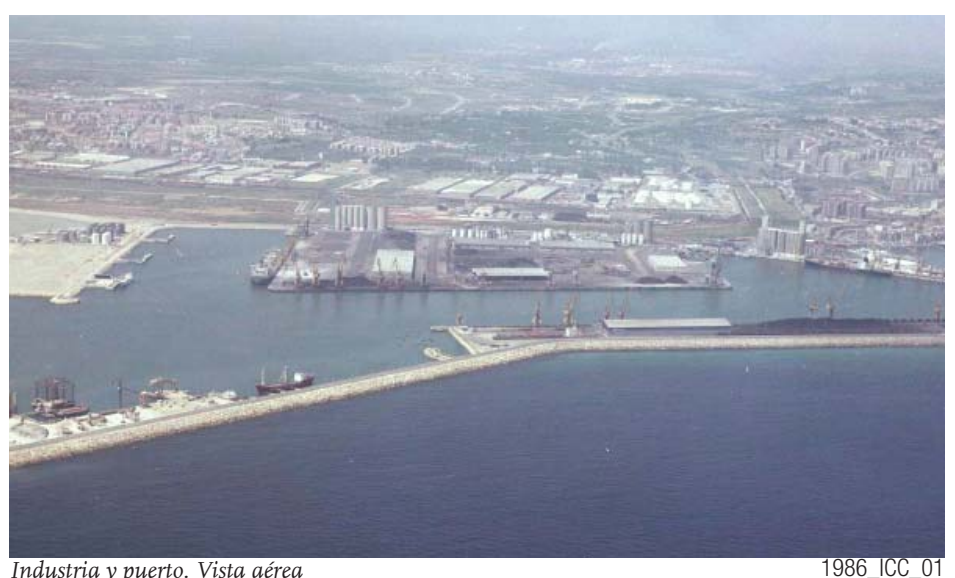

Instria $y$ puerto. Vista aérea

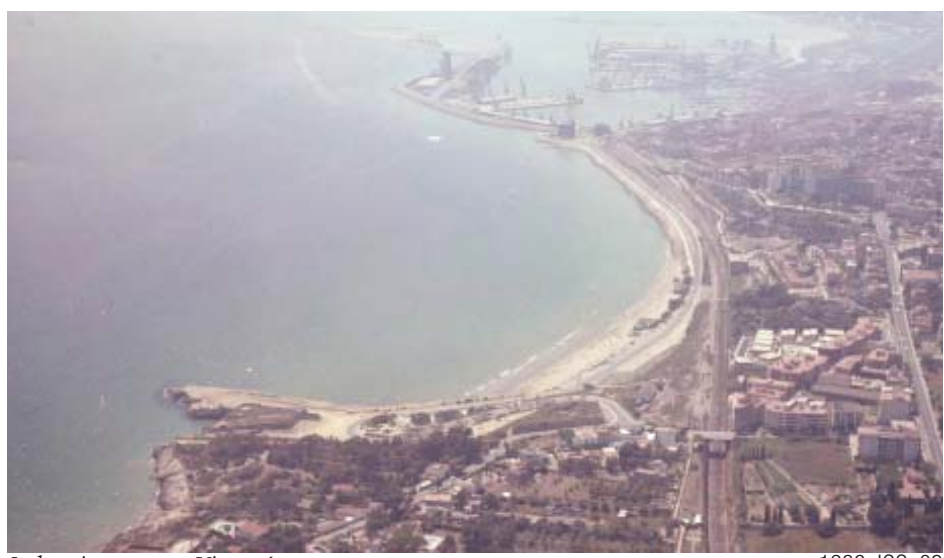
Industria y puerto. Vista aérea
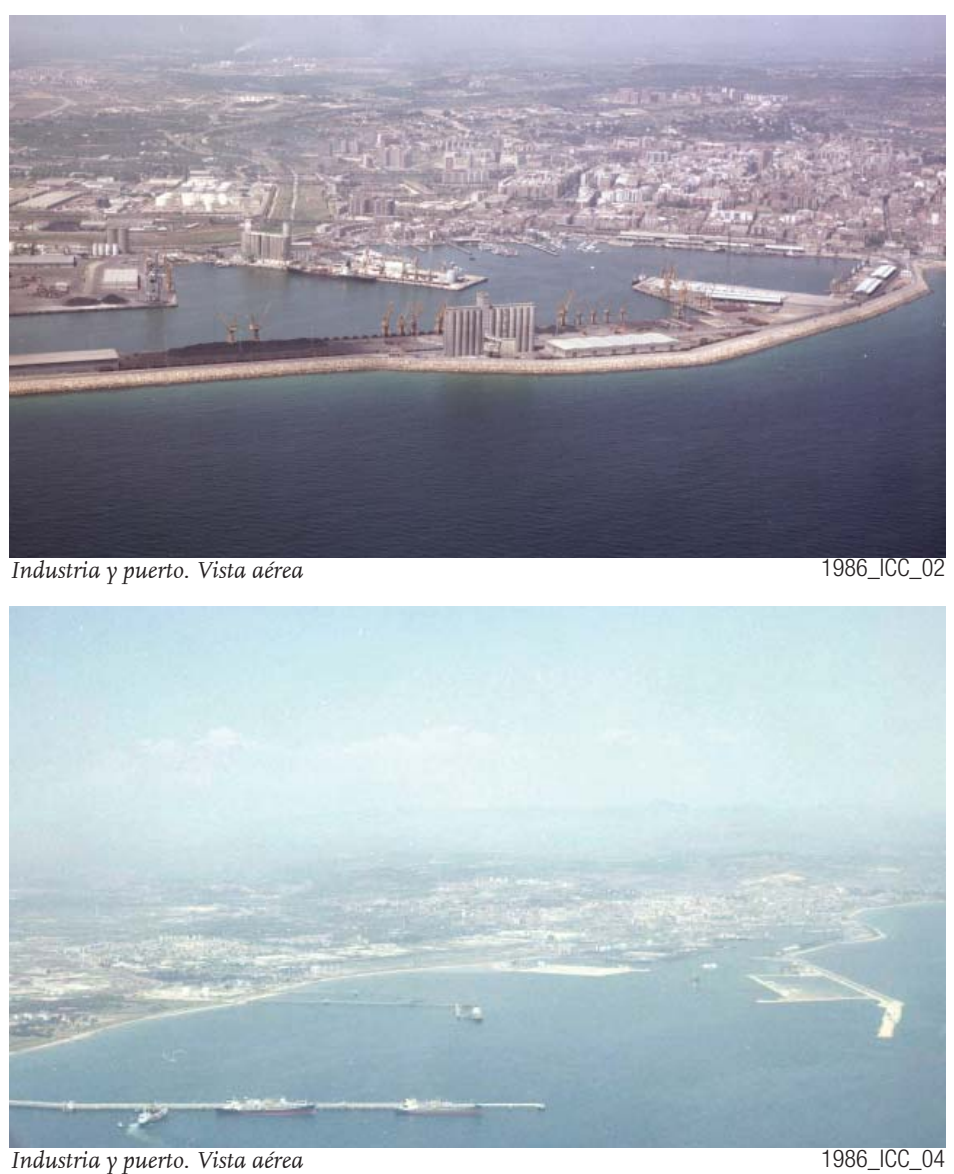


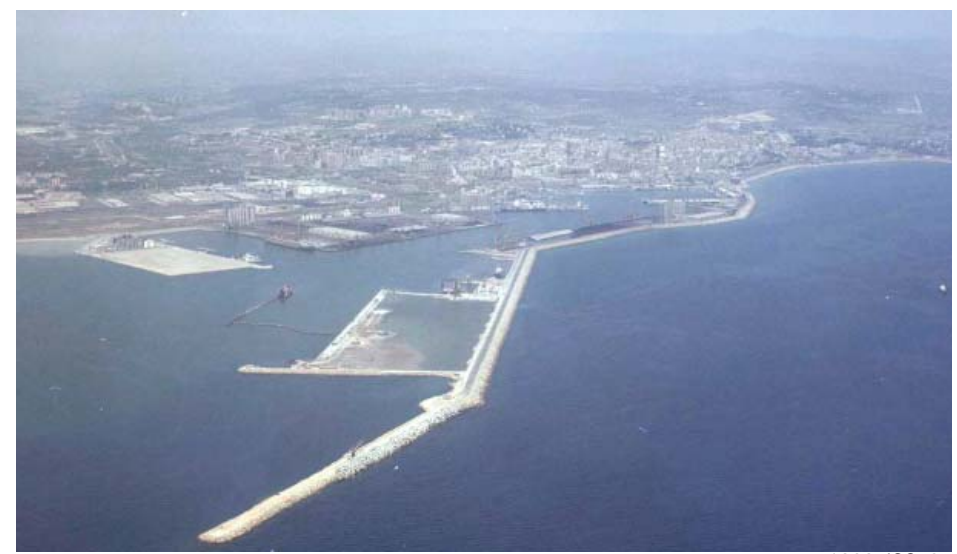

Industria Y puerto. Vista aérea

1986_ICC_05
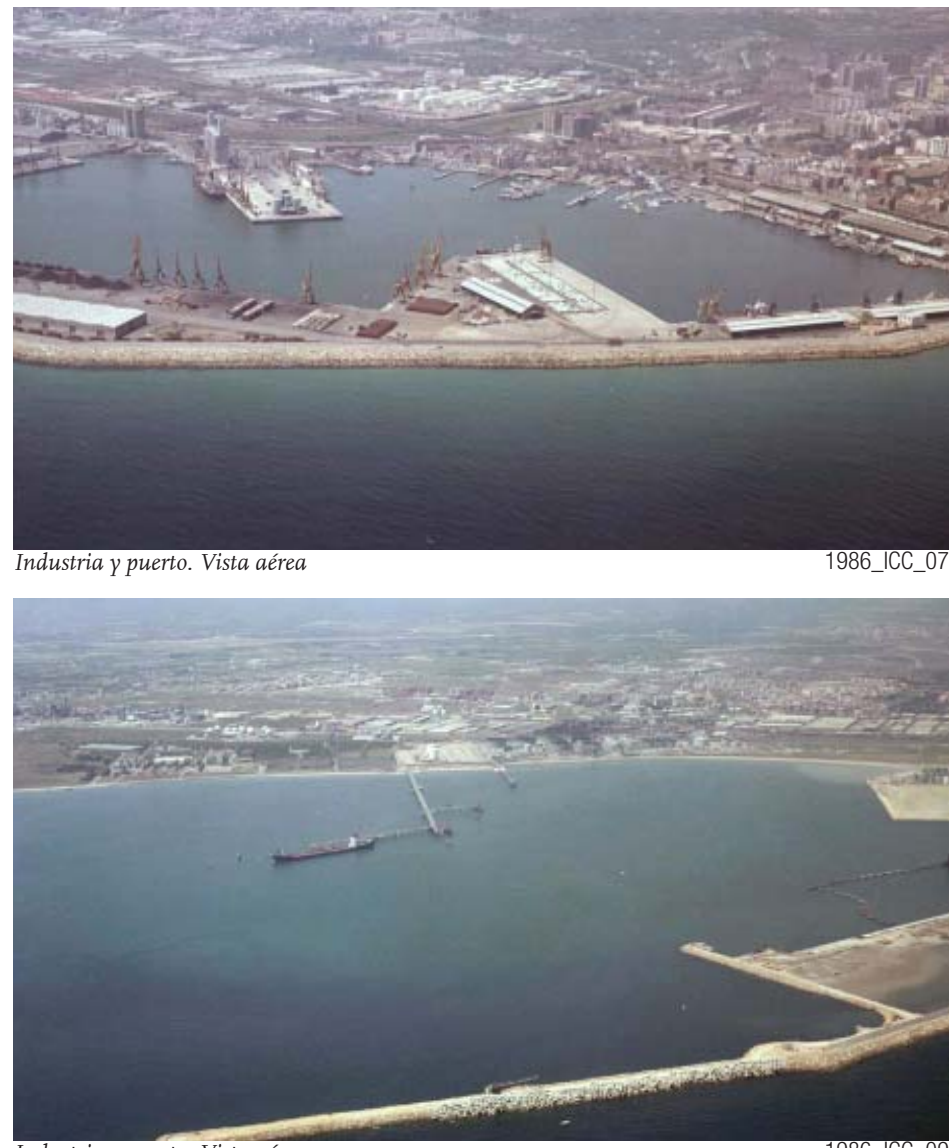

1986_ICC_09

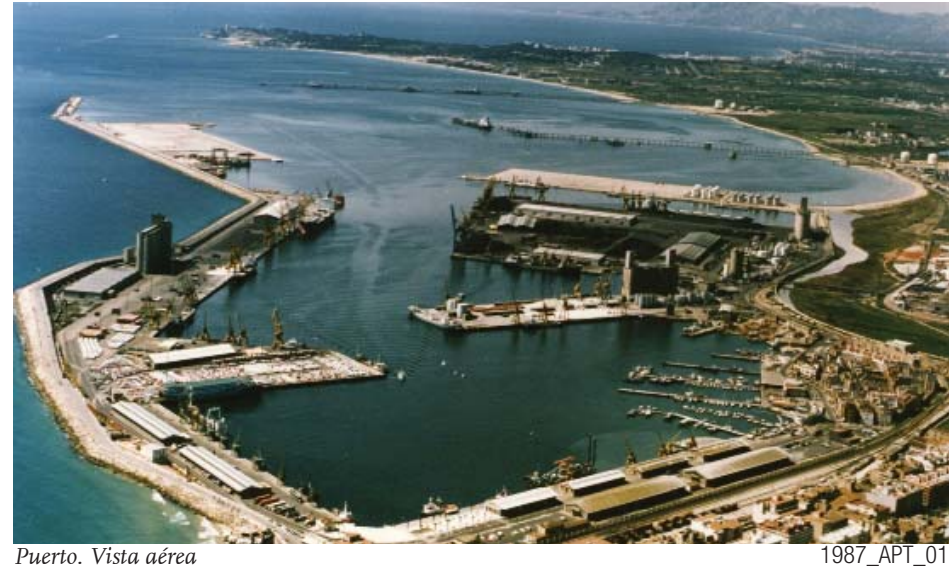

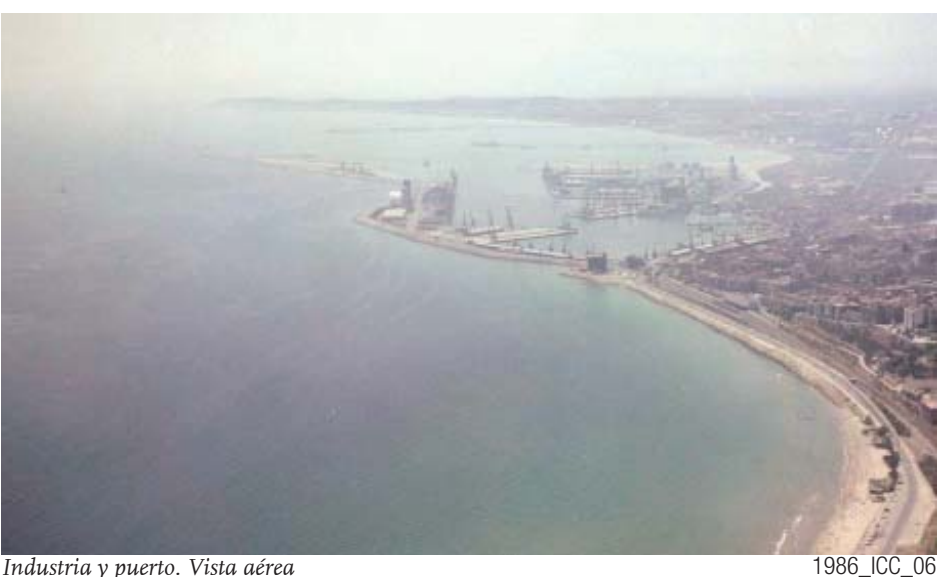
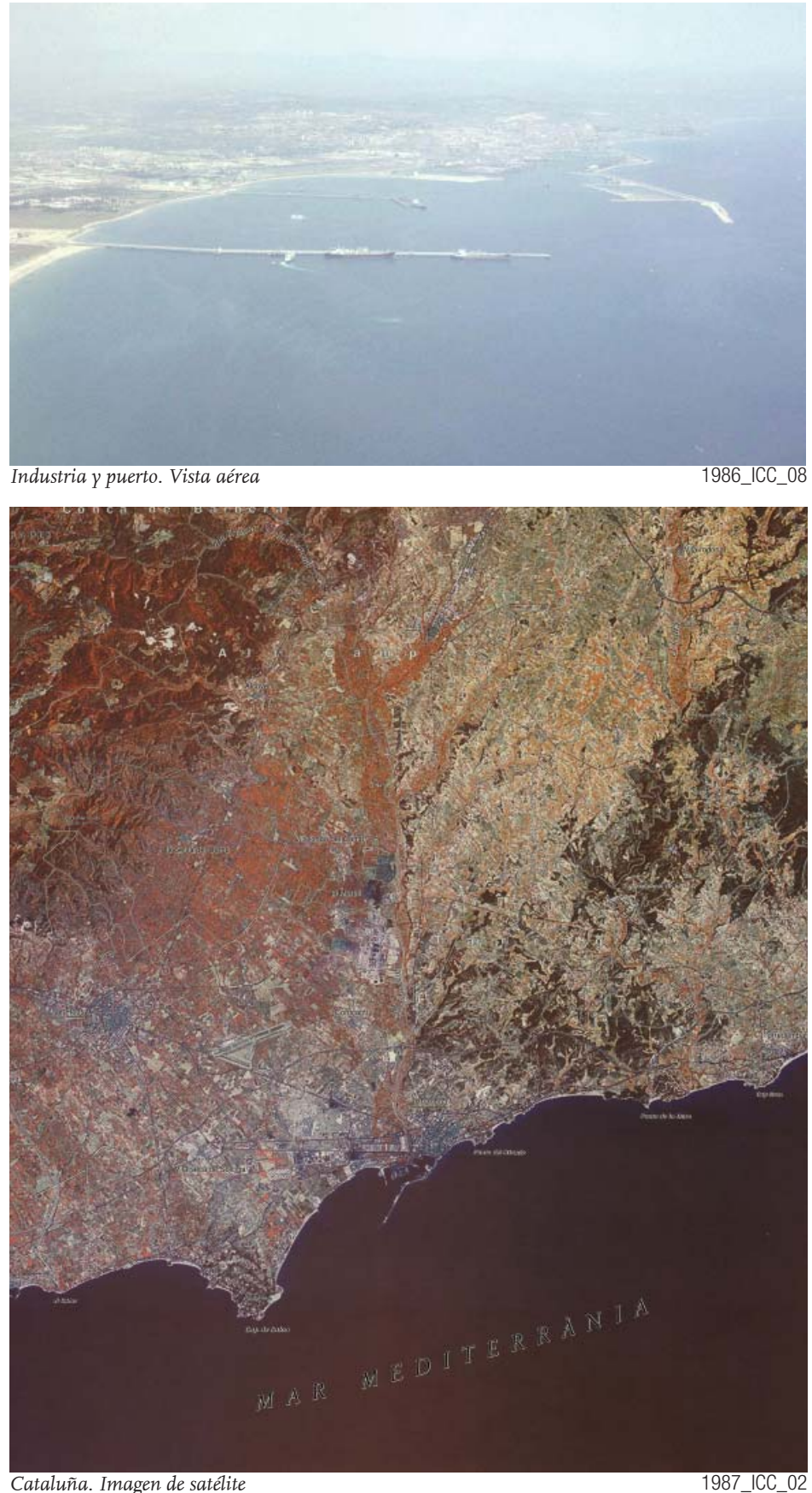


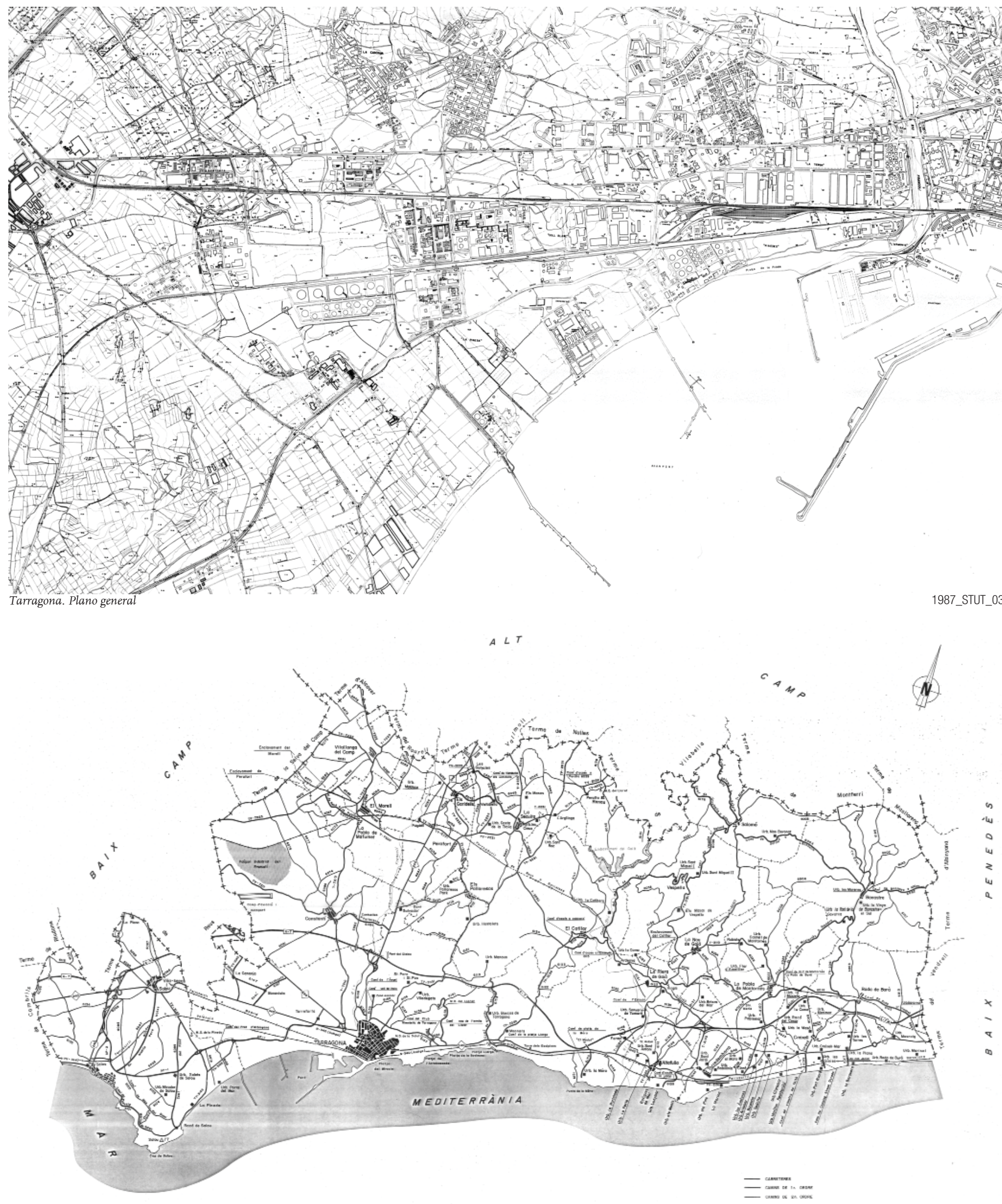



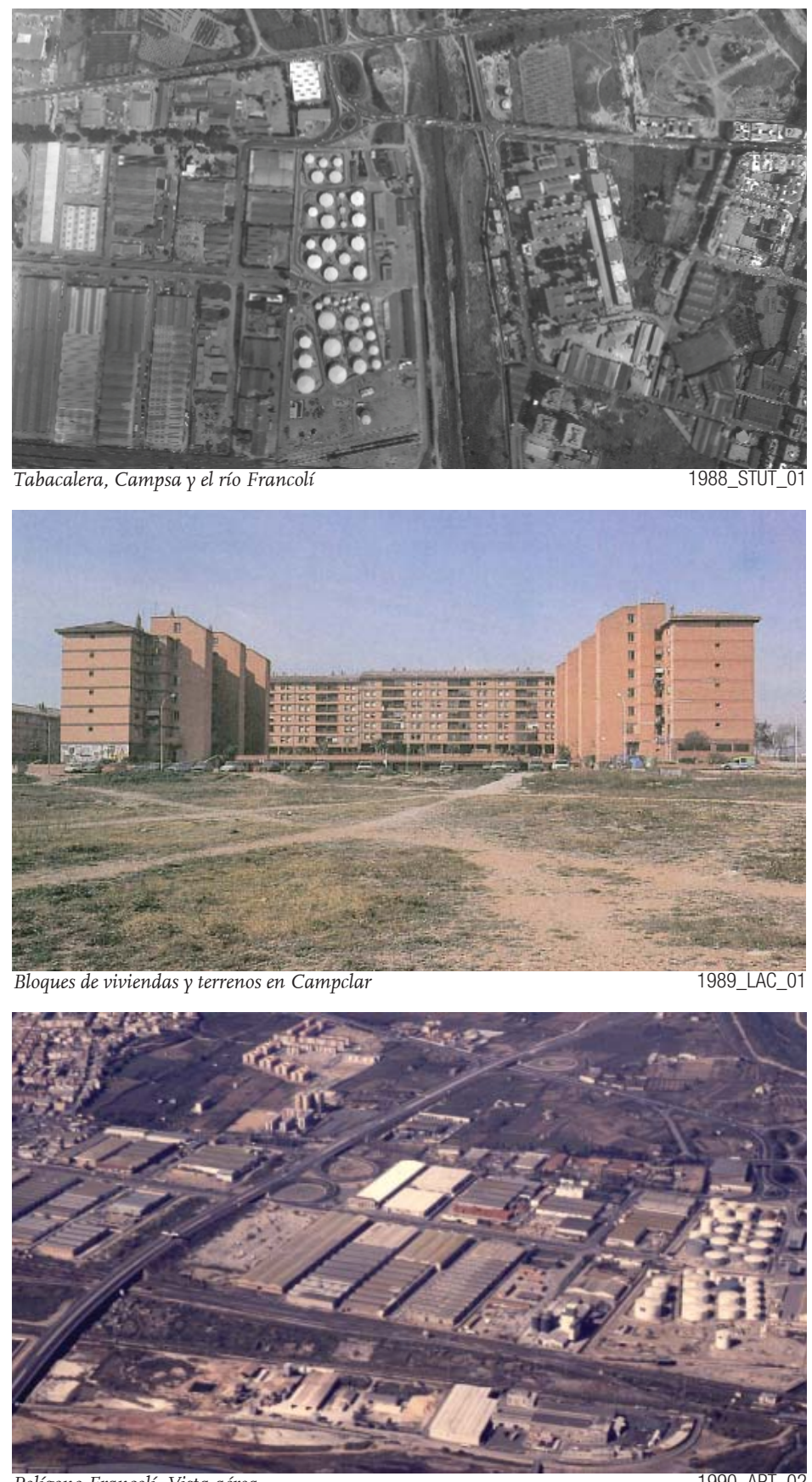

Polígono Francolí. Vista aérea

1990_APT_02

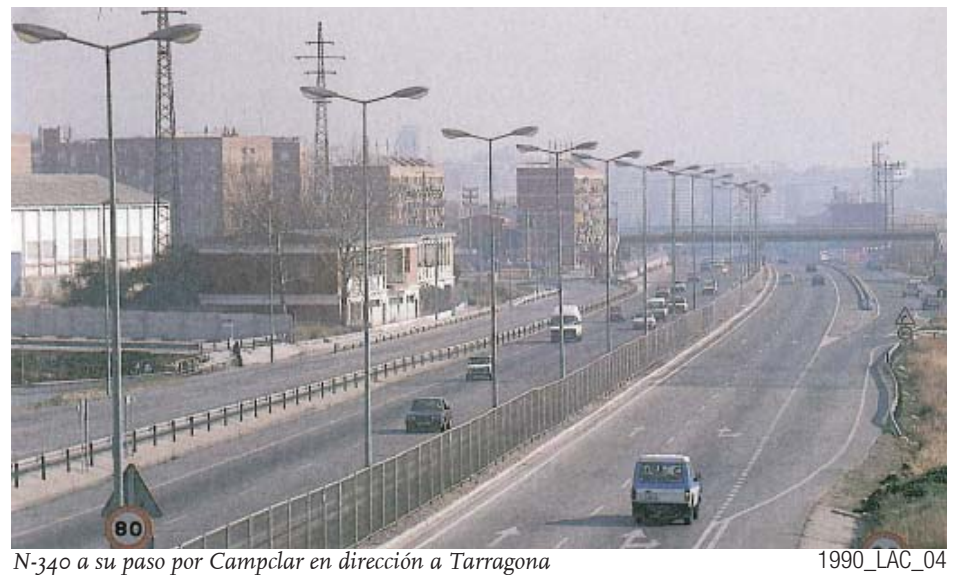

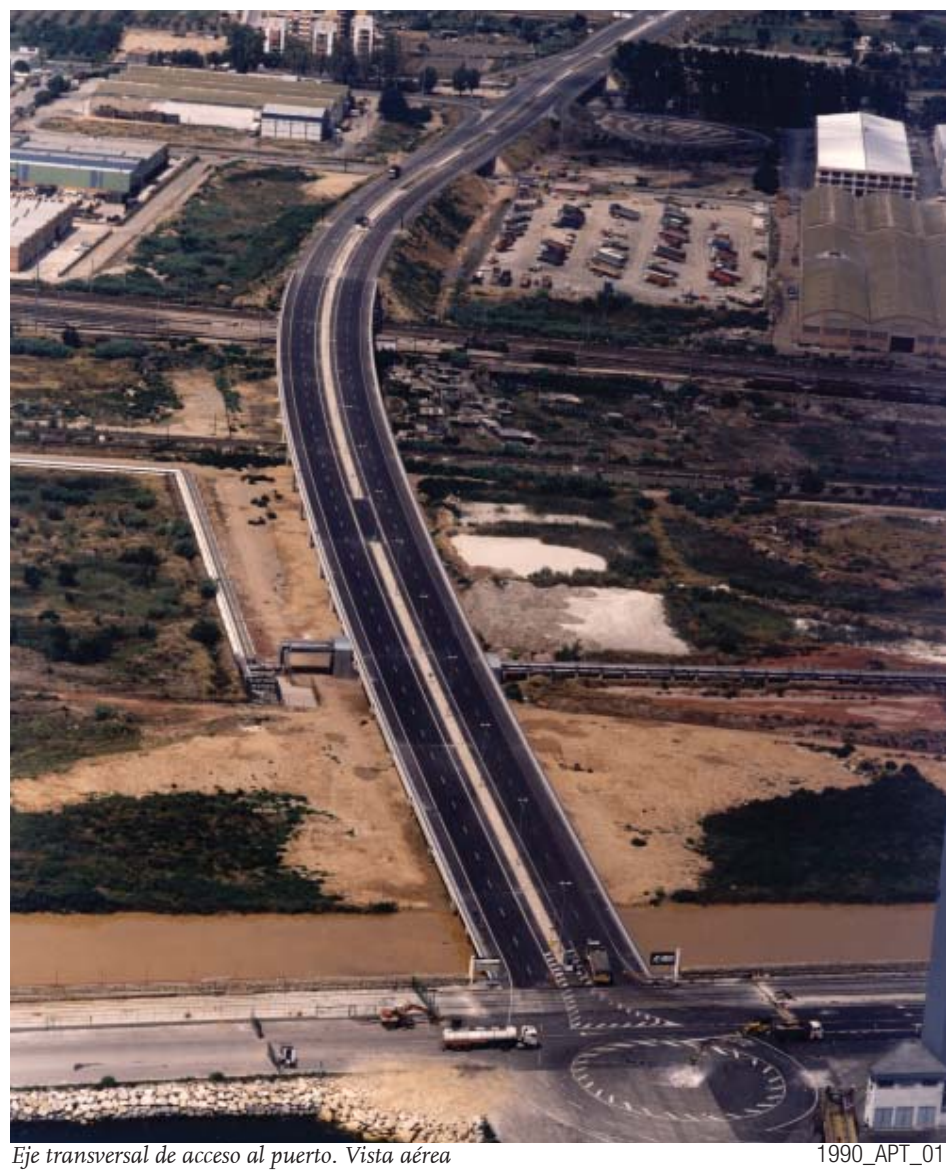
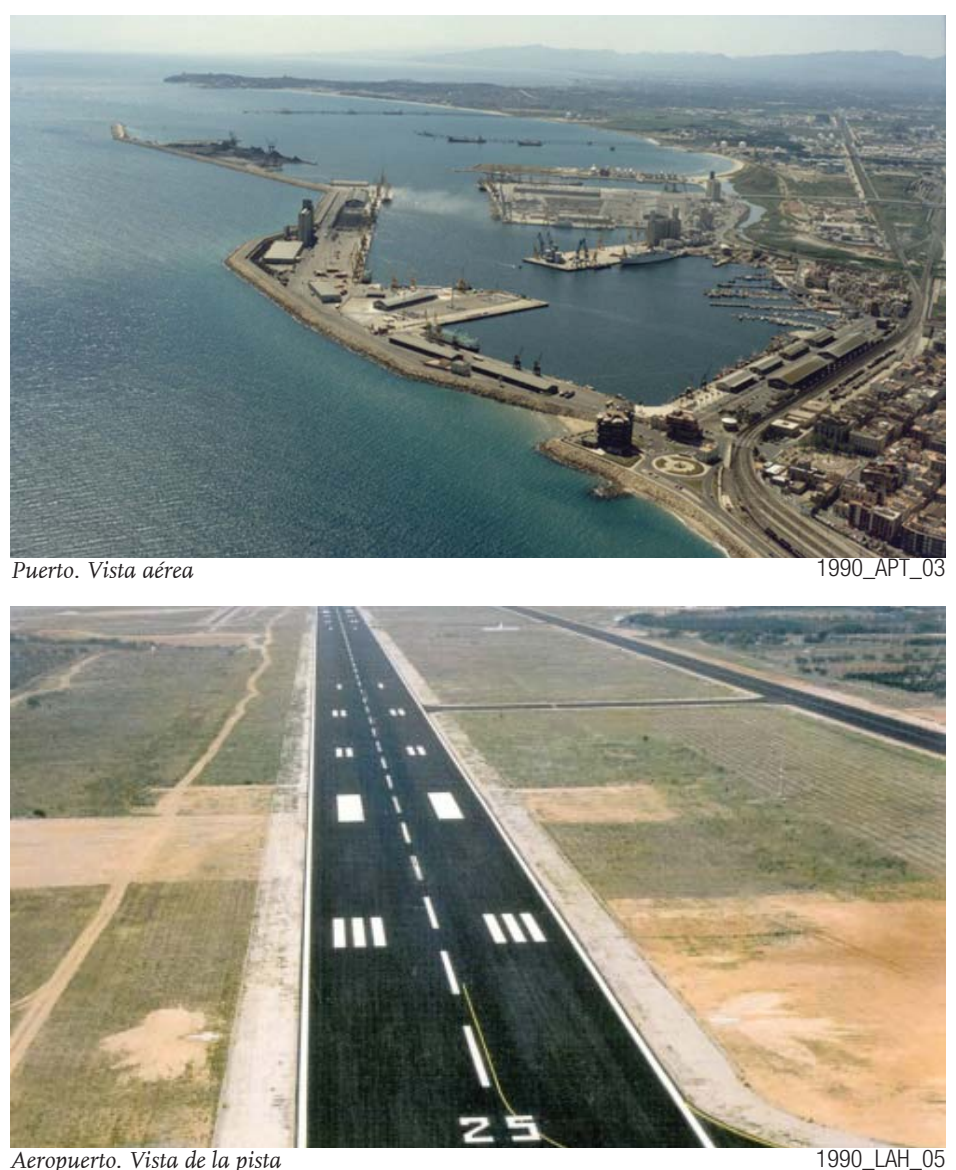

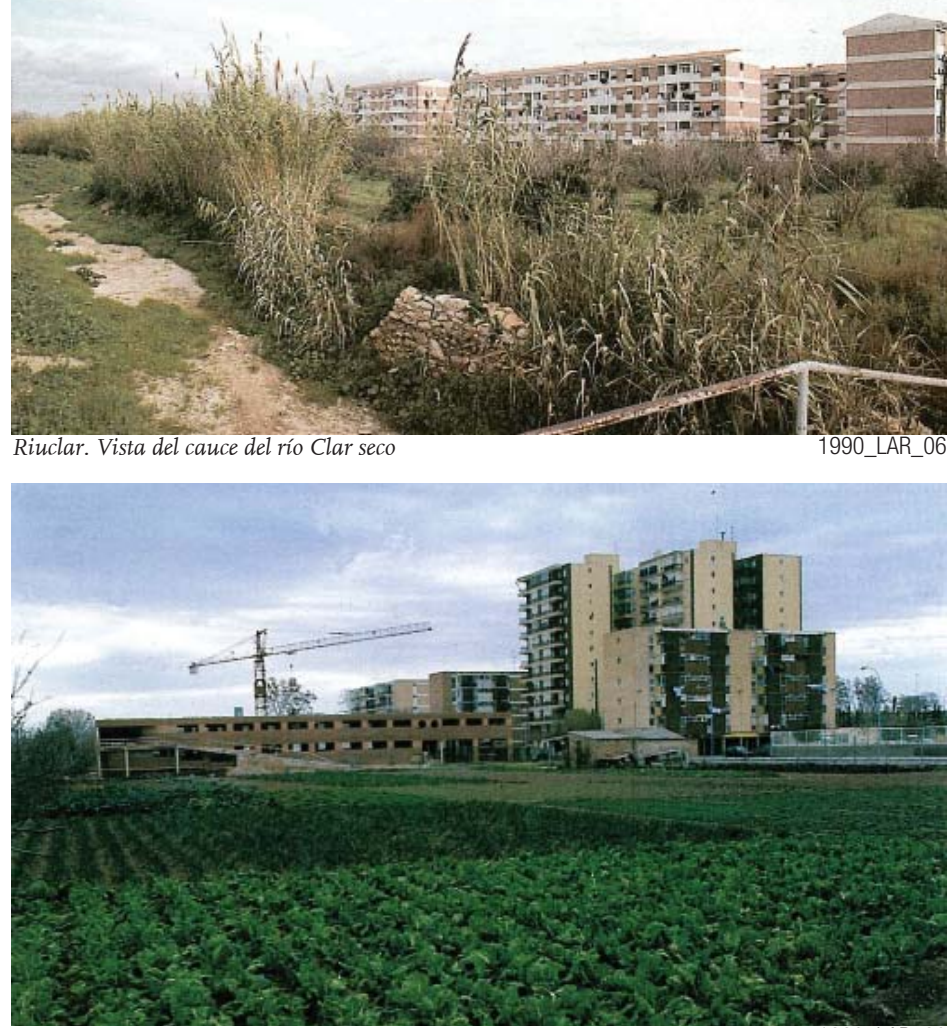

Riuclar. Vista de la Horta Gran que rodea al barrio Icomar

1990_LAR_08

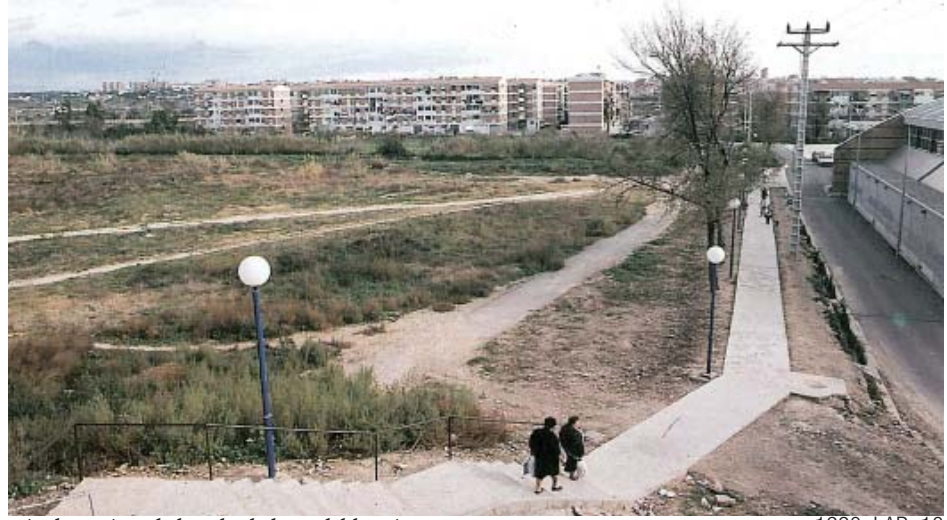

Riuclar. Vista de los alrededores del barrio

990_LAR_10

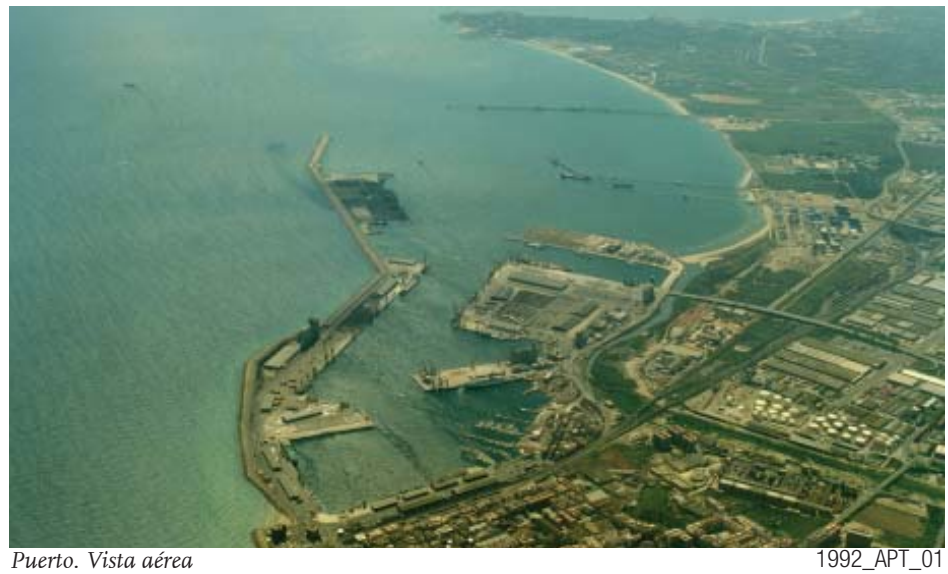

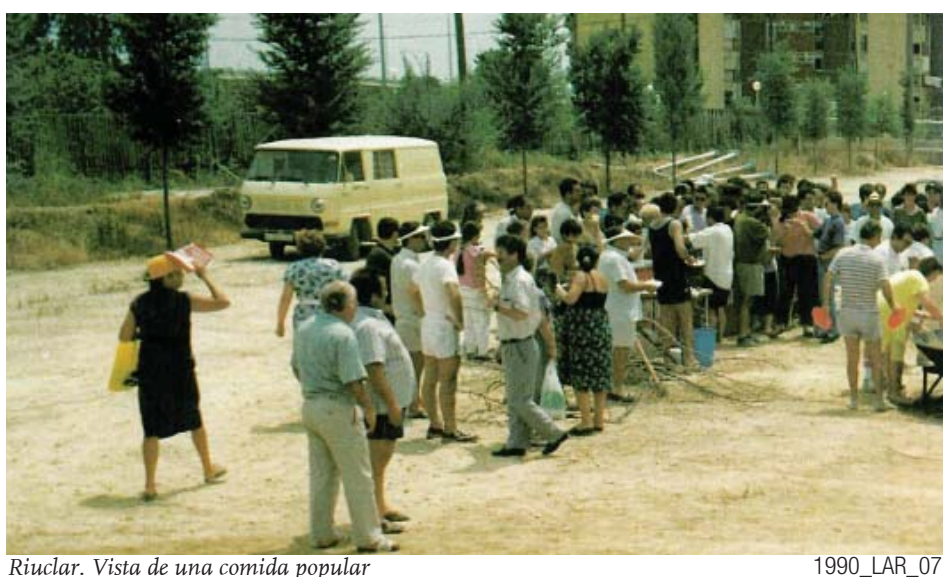
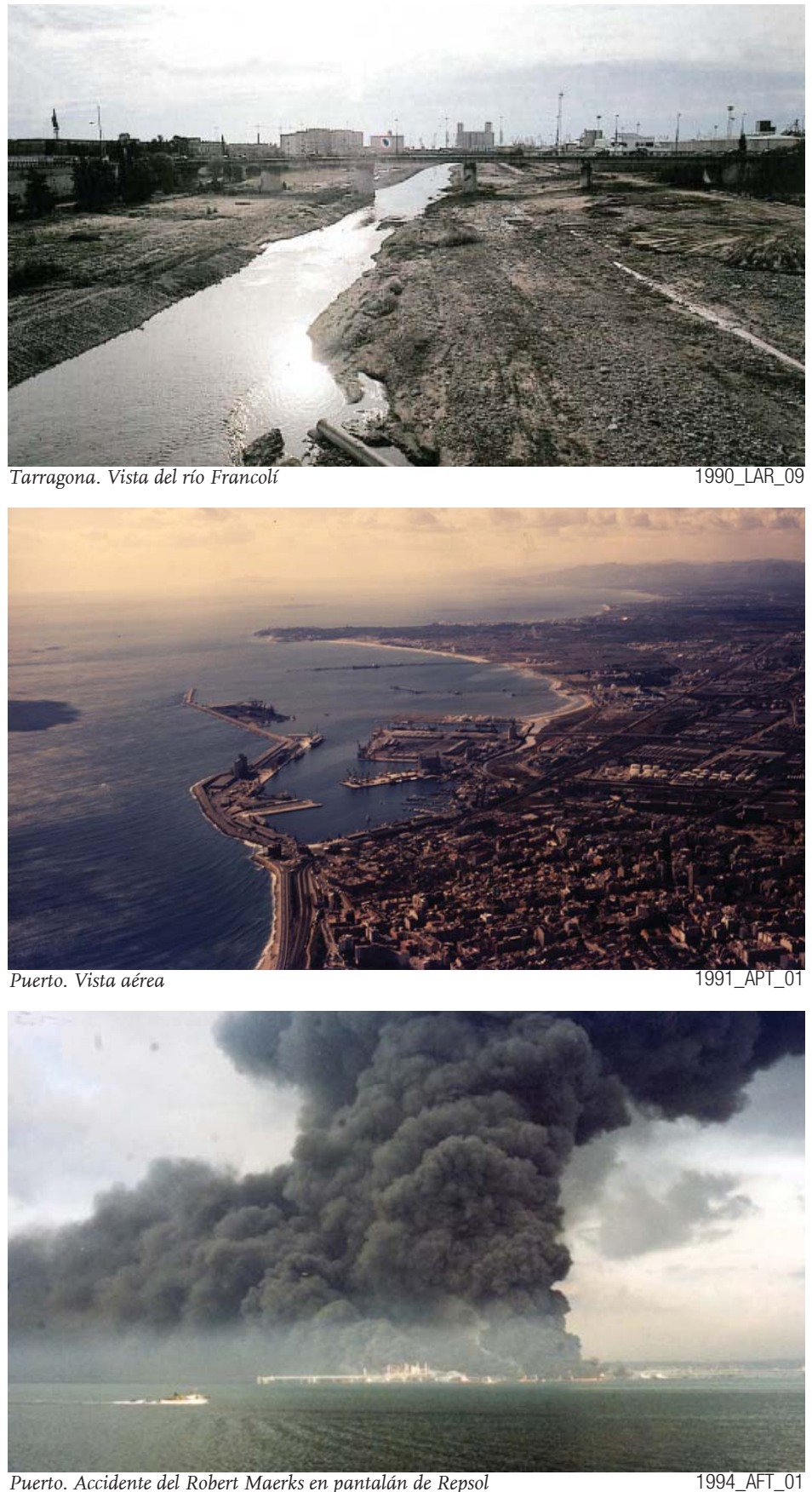

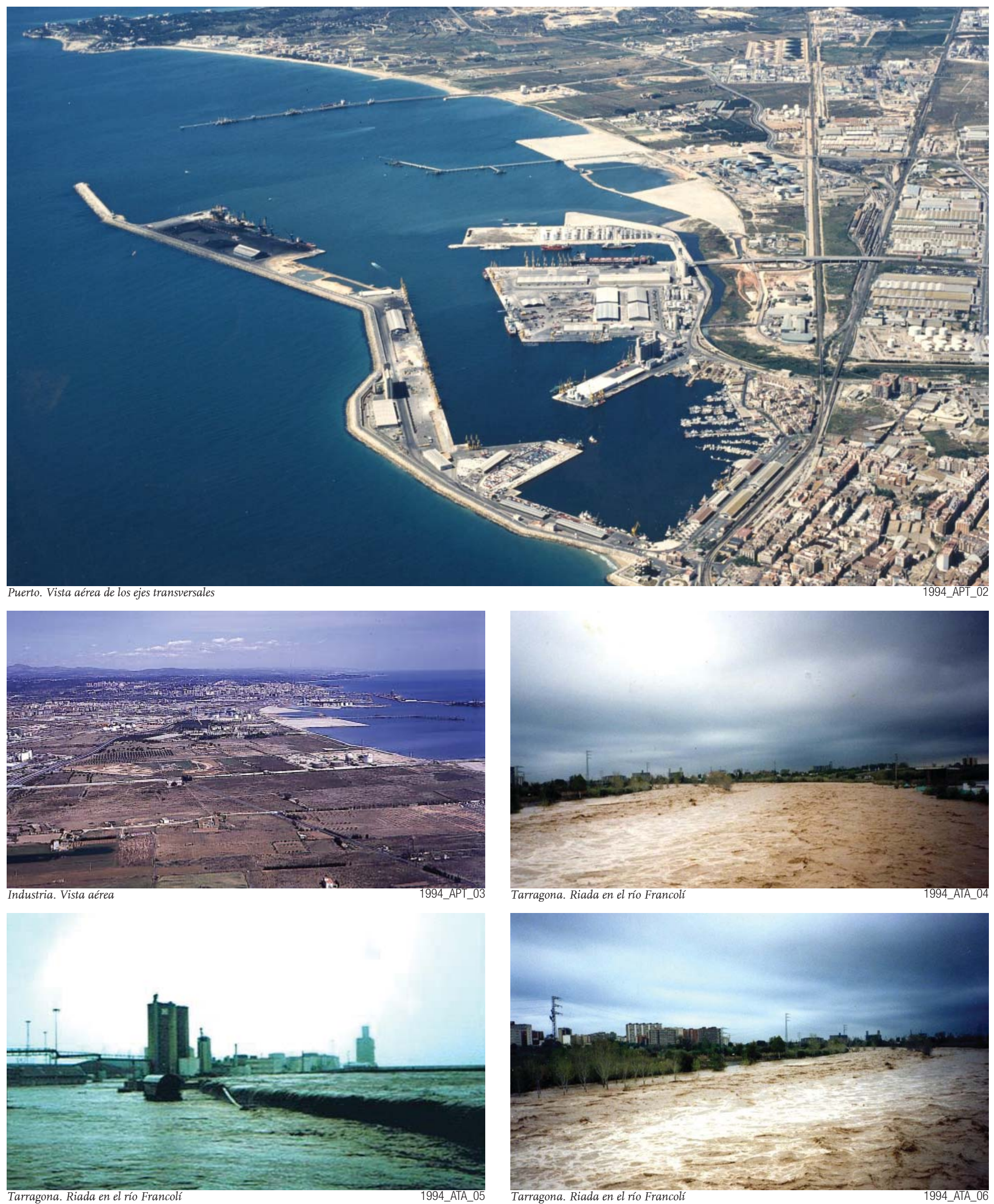


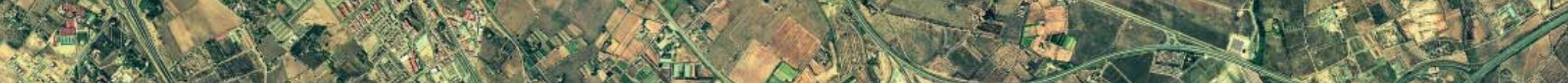

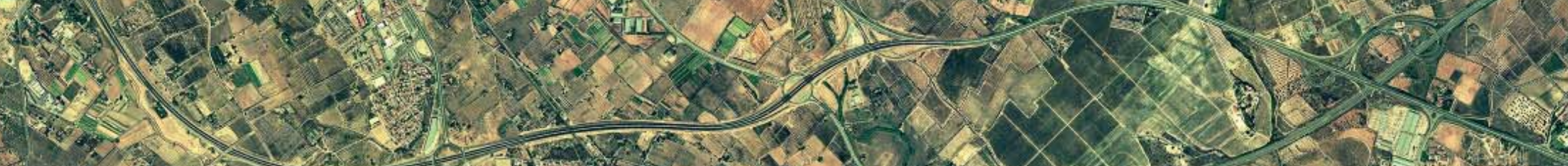

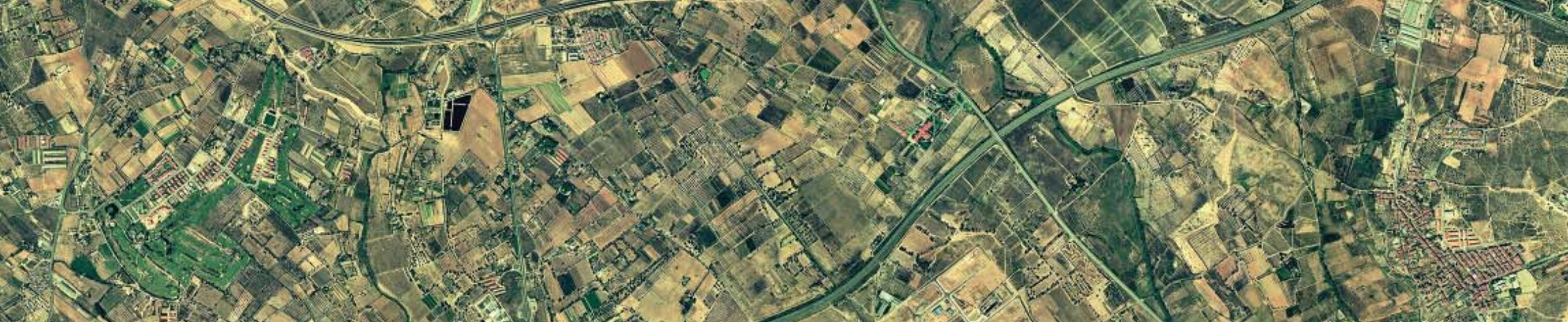

$3+2=2,20$

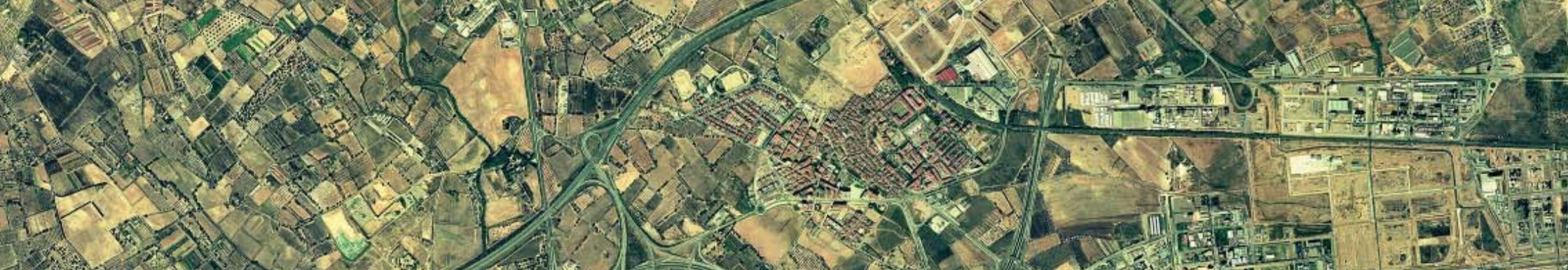

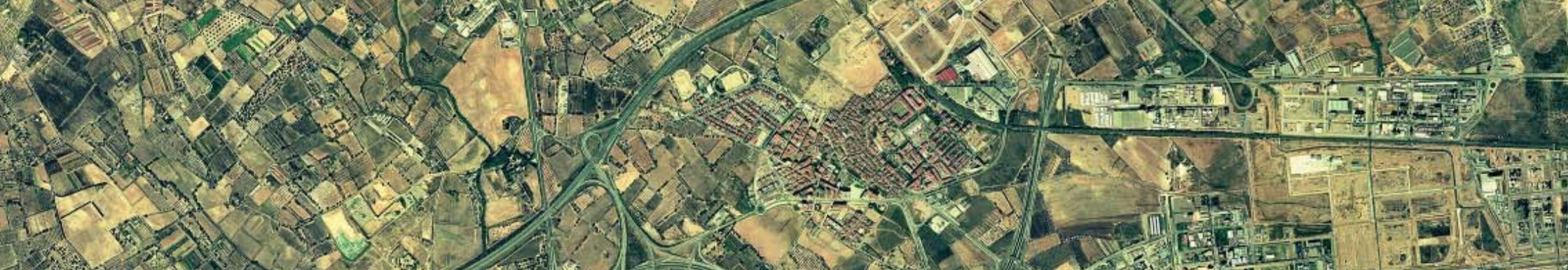

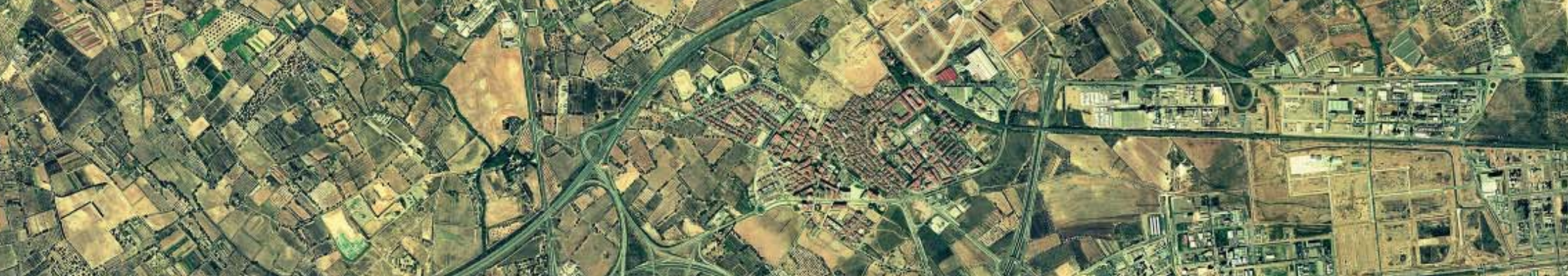

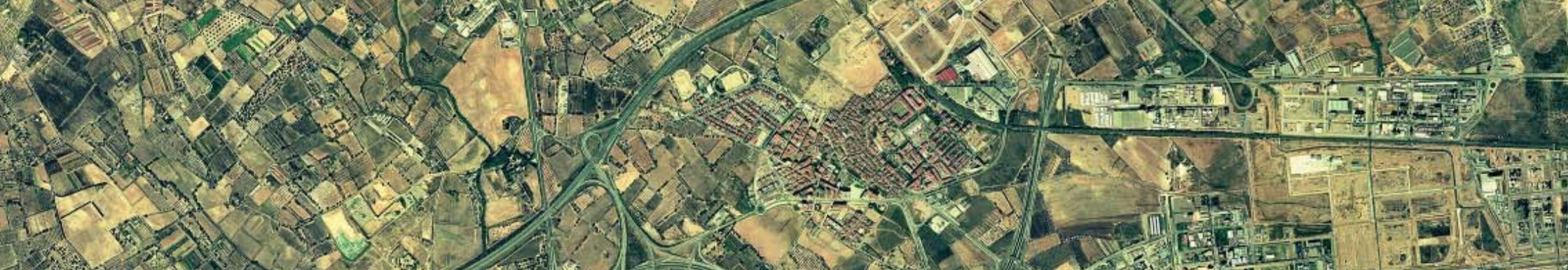

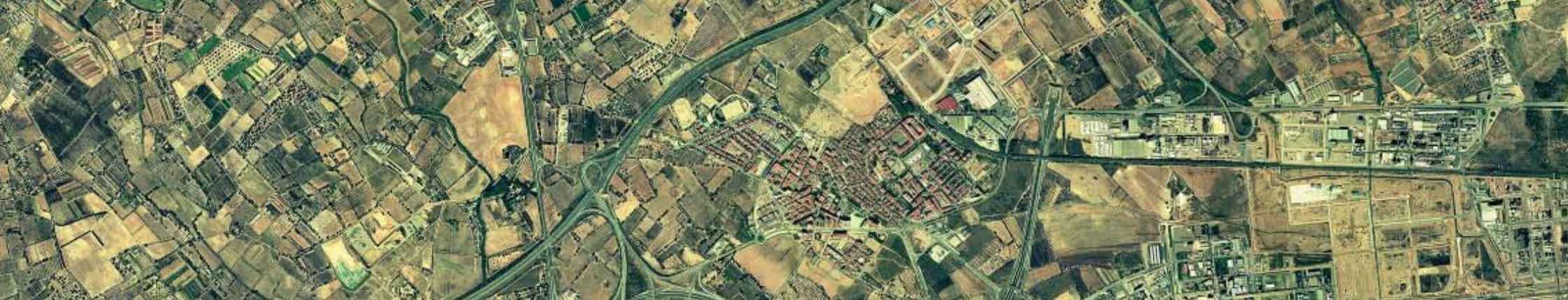

ary $x$ ?

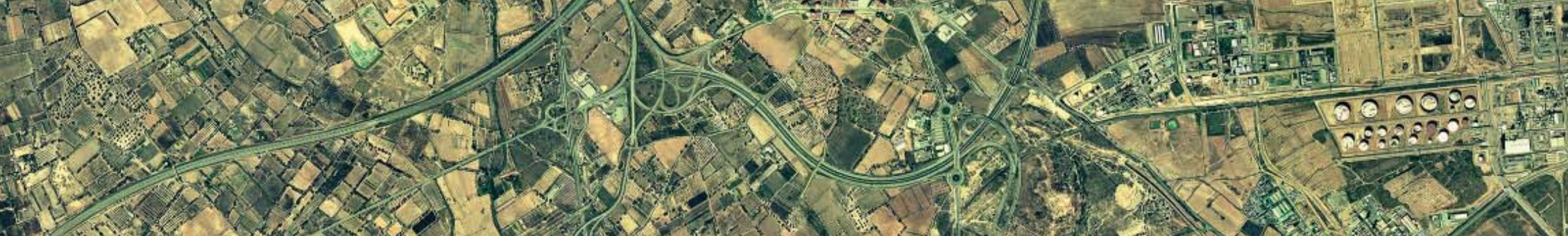

- $2.2,2,16$

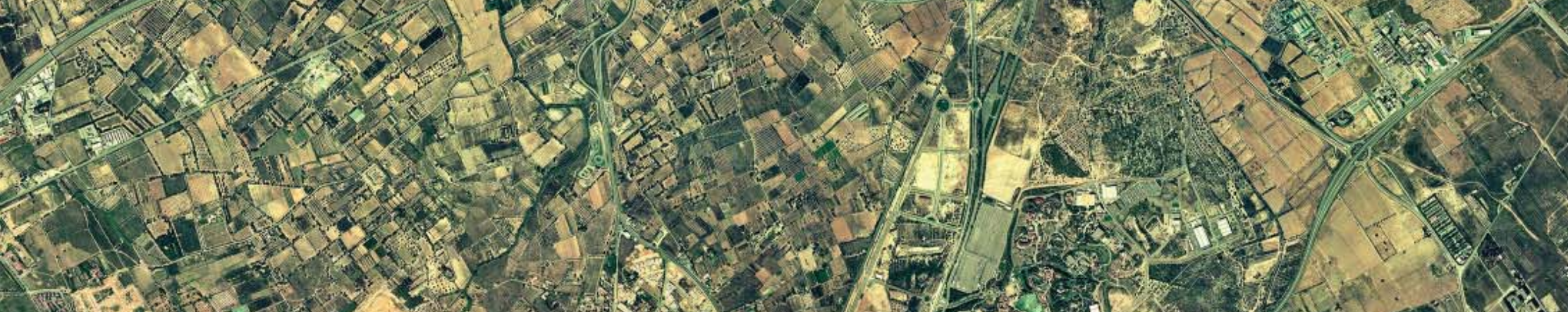
- z

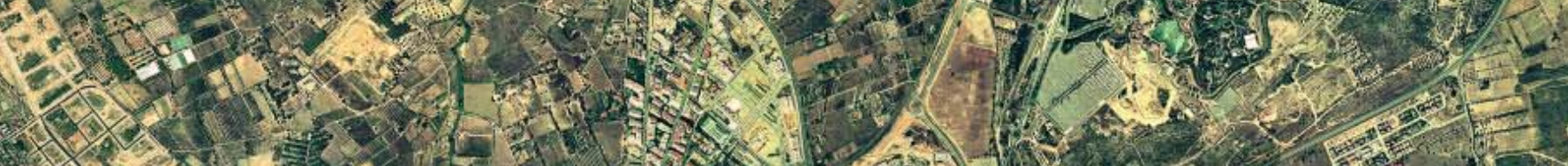

- 20 .

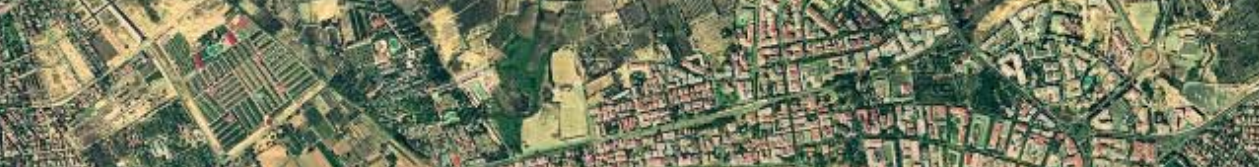

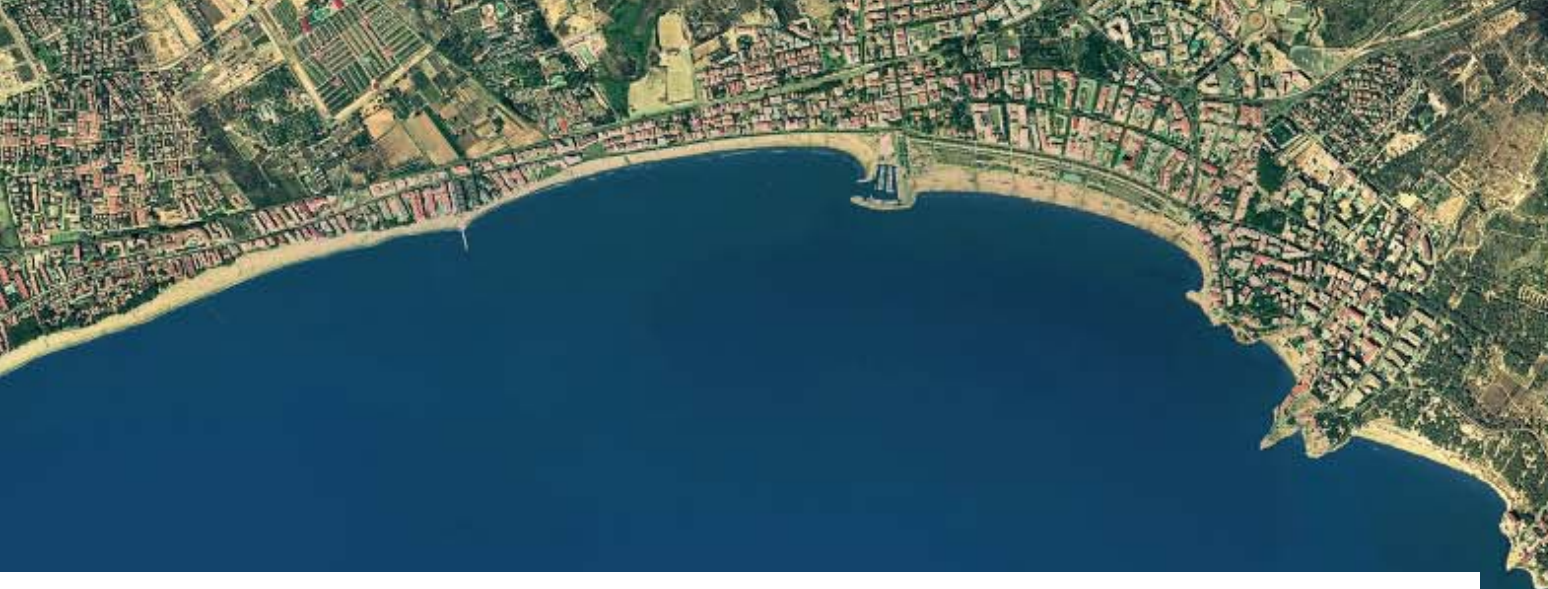

Tarragona. Ortofoto

$\stackrel{2}{200} \check{5}^{500}(1)$

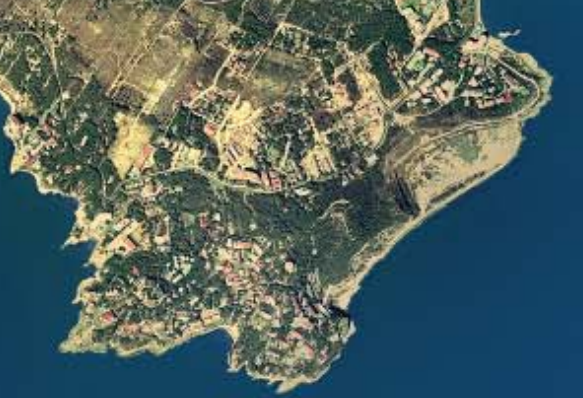




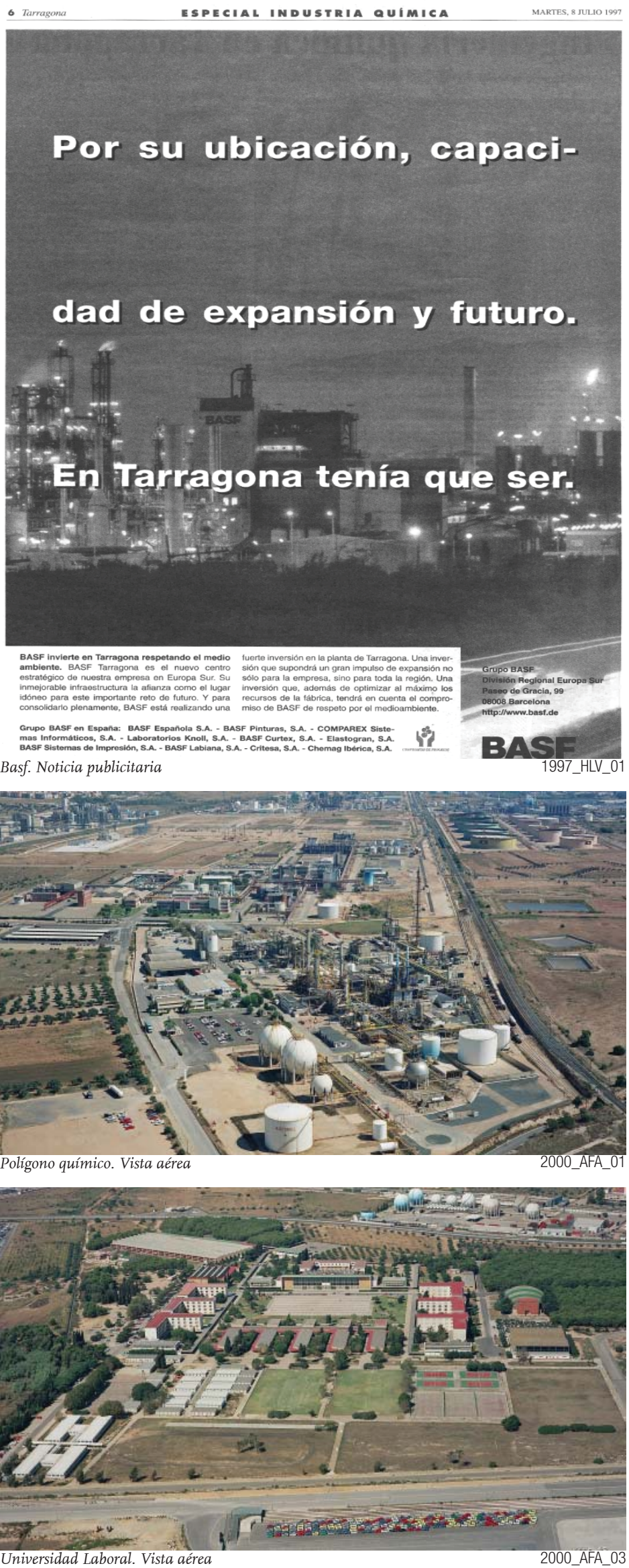

Basf se expande para ganar en competitividad y rentabilidad
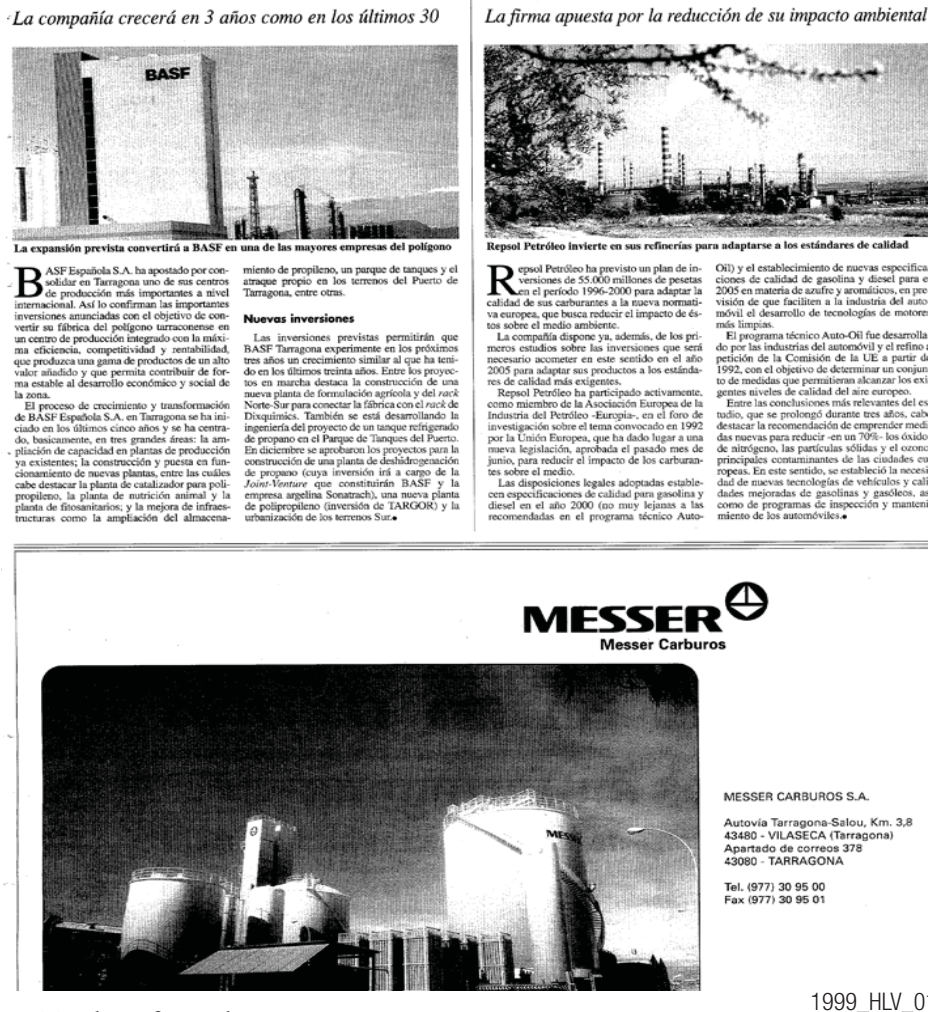

Noticias de Basf, Repsol y Messer
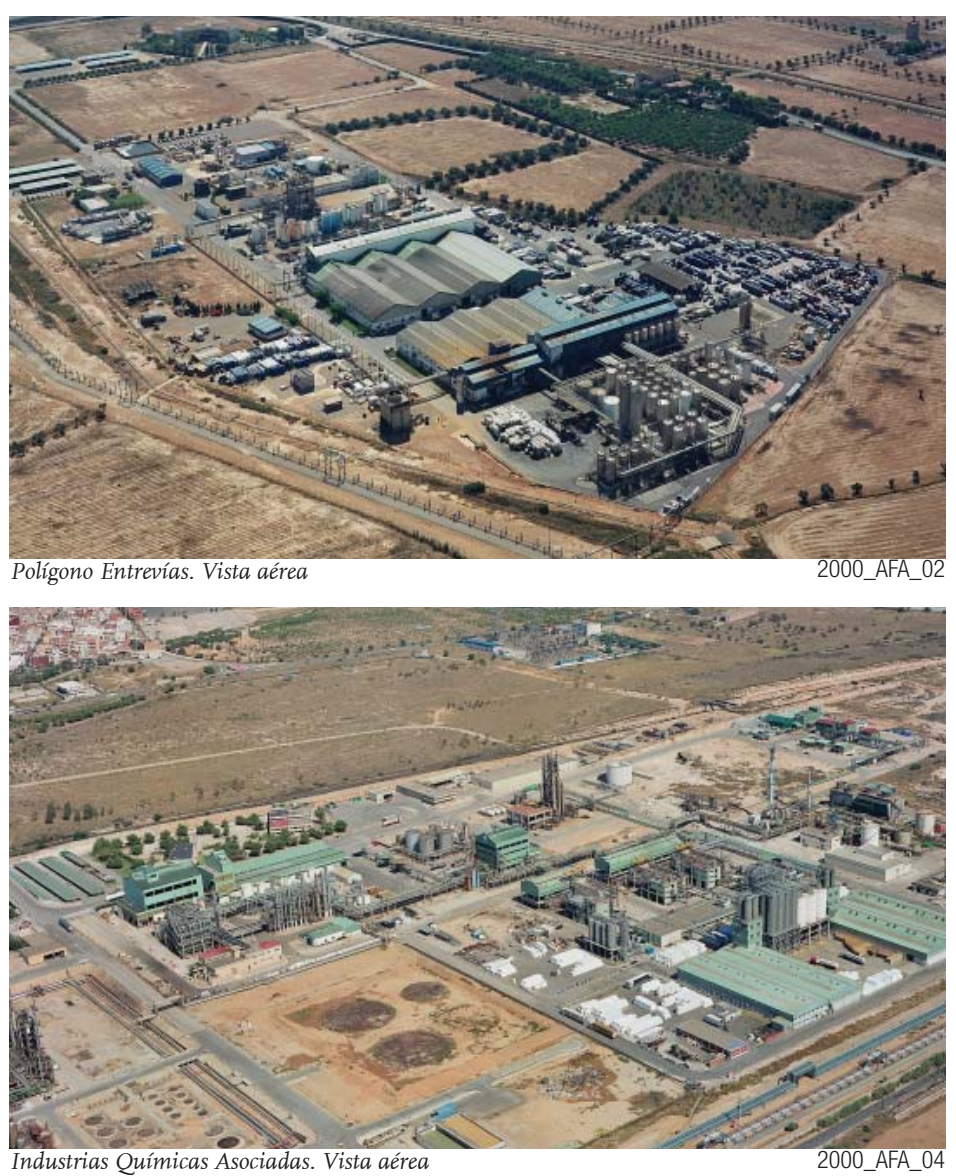

Repsol invierte 55.000 millones para unos carburantes limpios
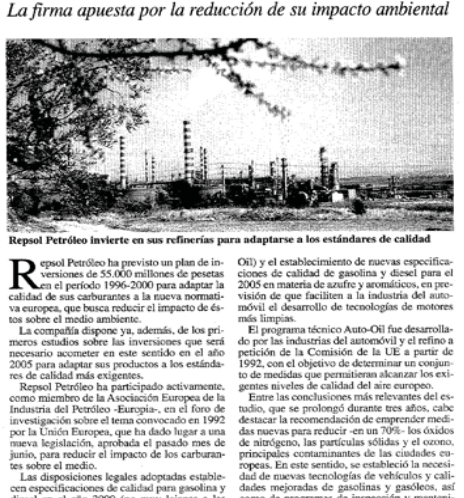

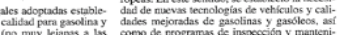

\section{MESSER ${ }^{\oplus}$}

999_HLV_0 

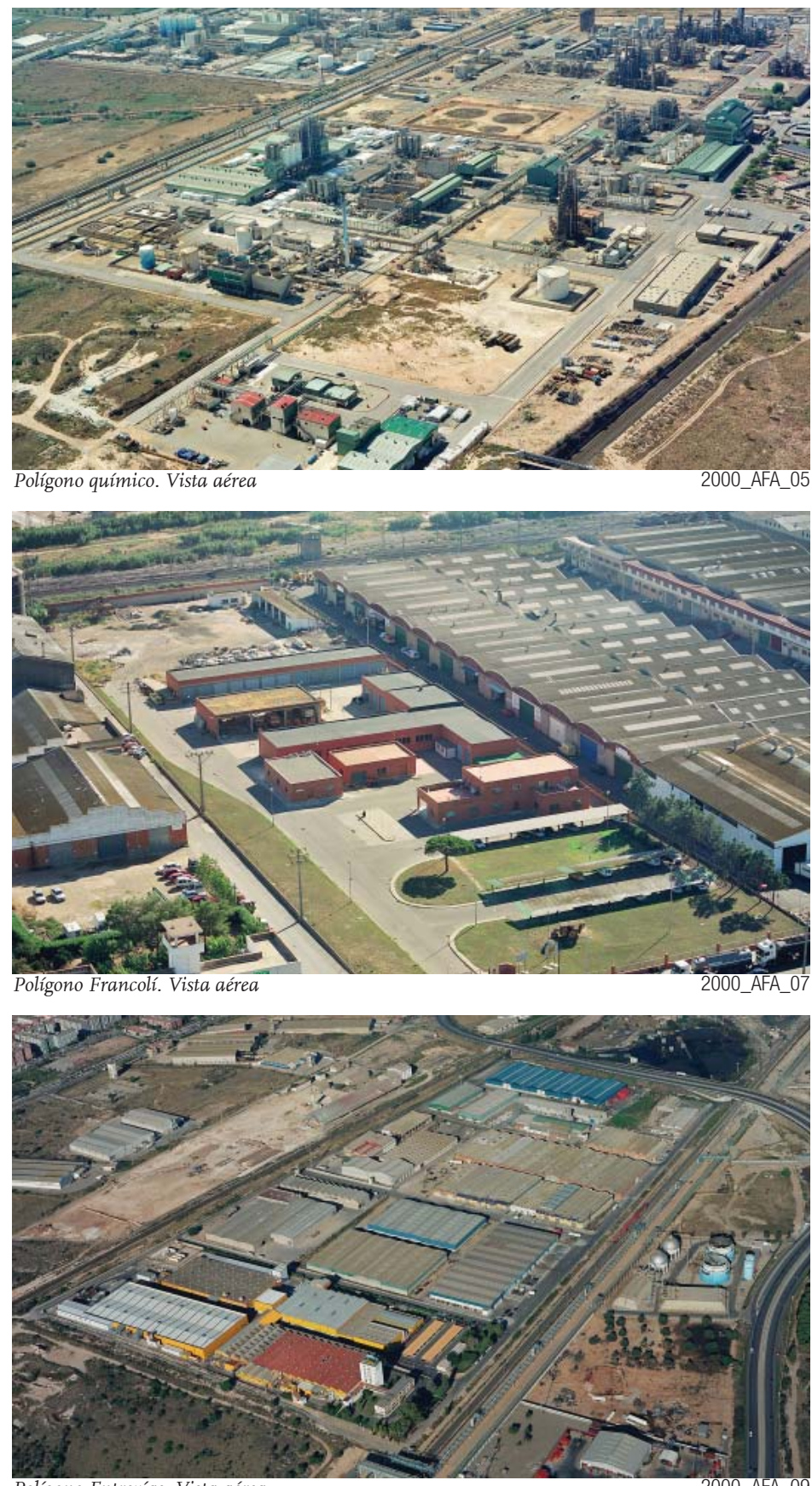

Polígono Entrevías. Vista aérea

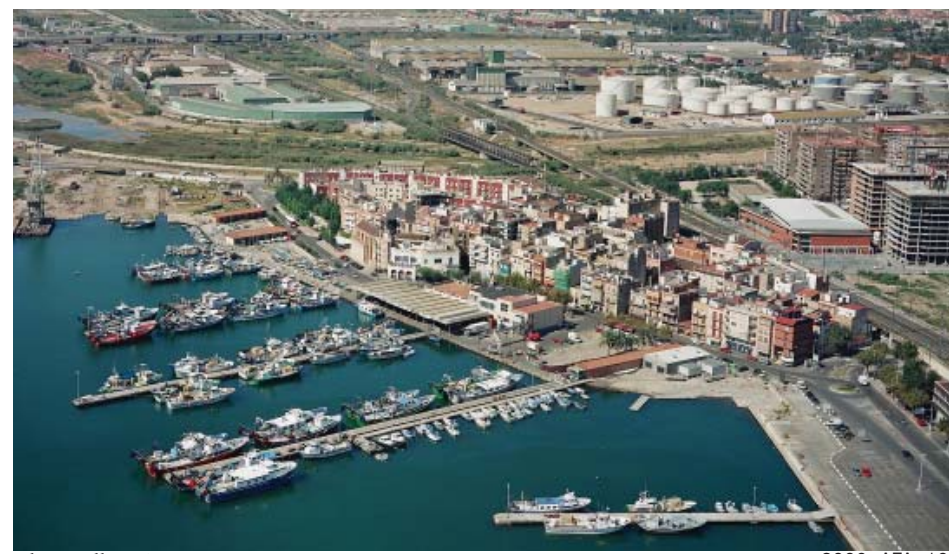

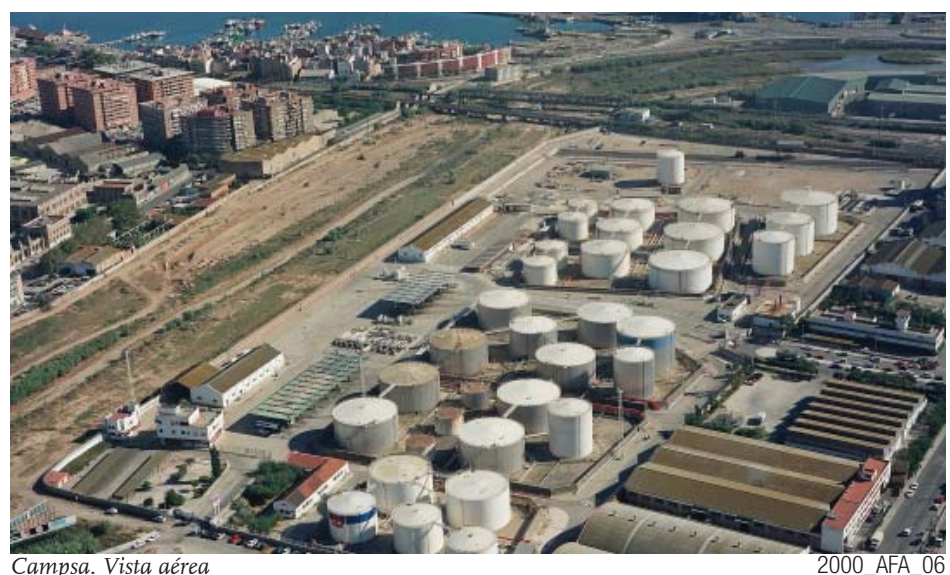
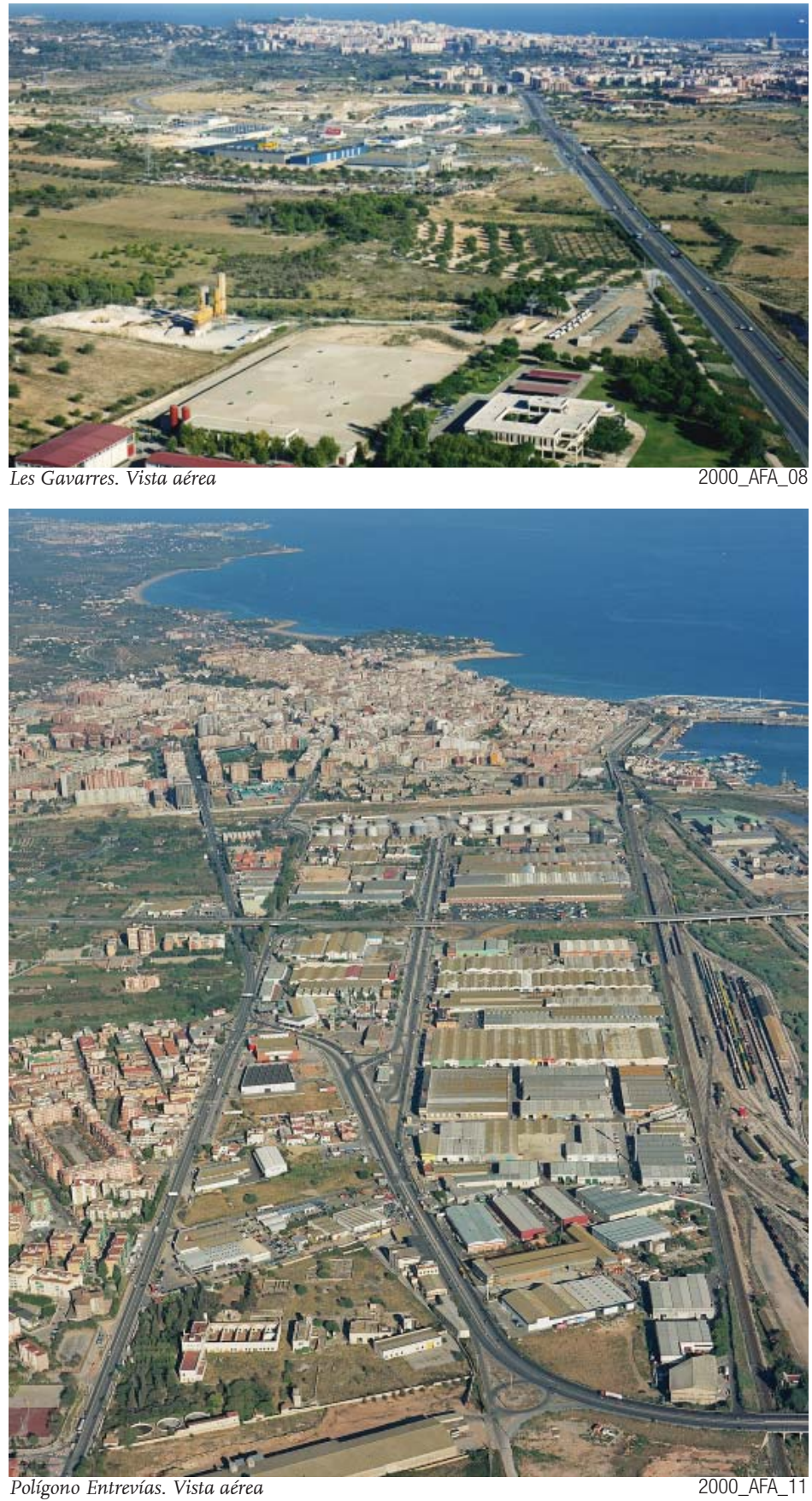


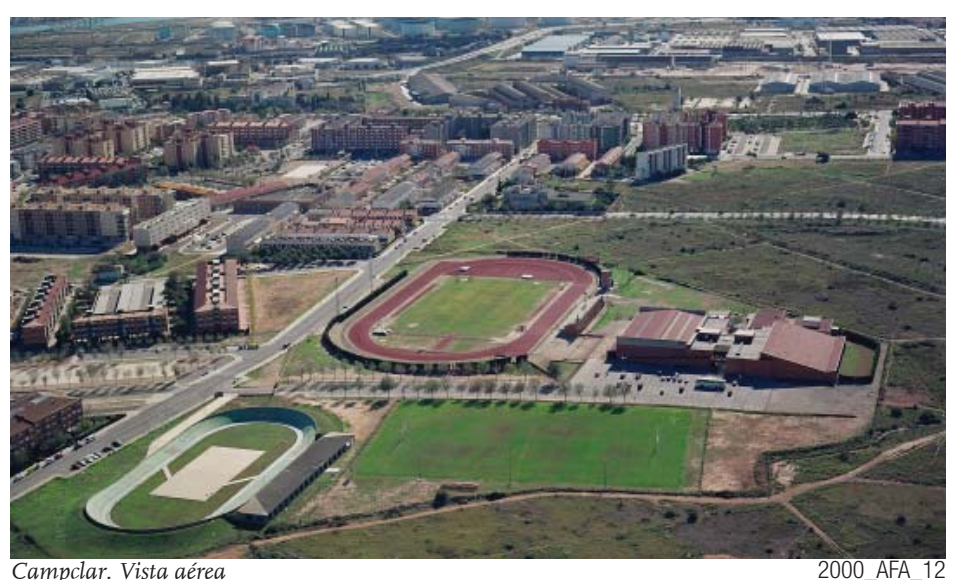

Campclar. Vista aérea
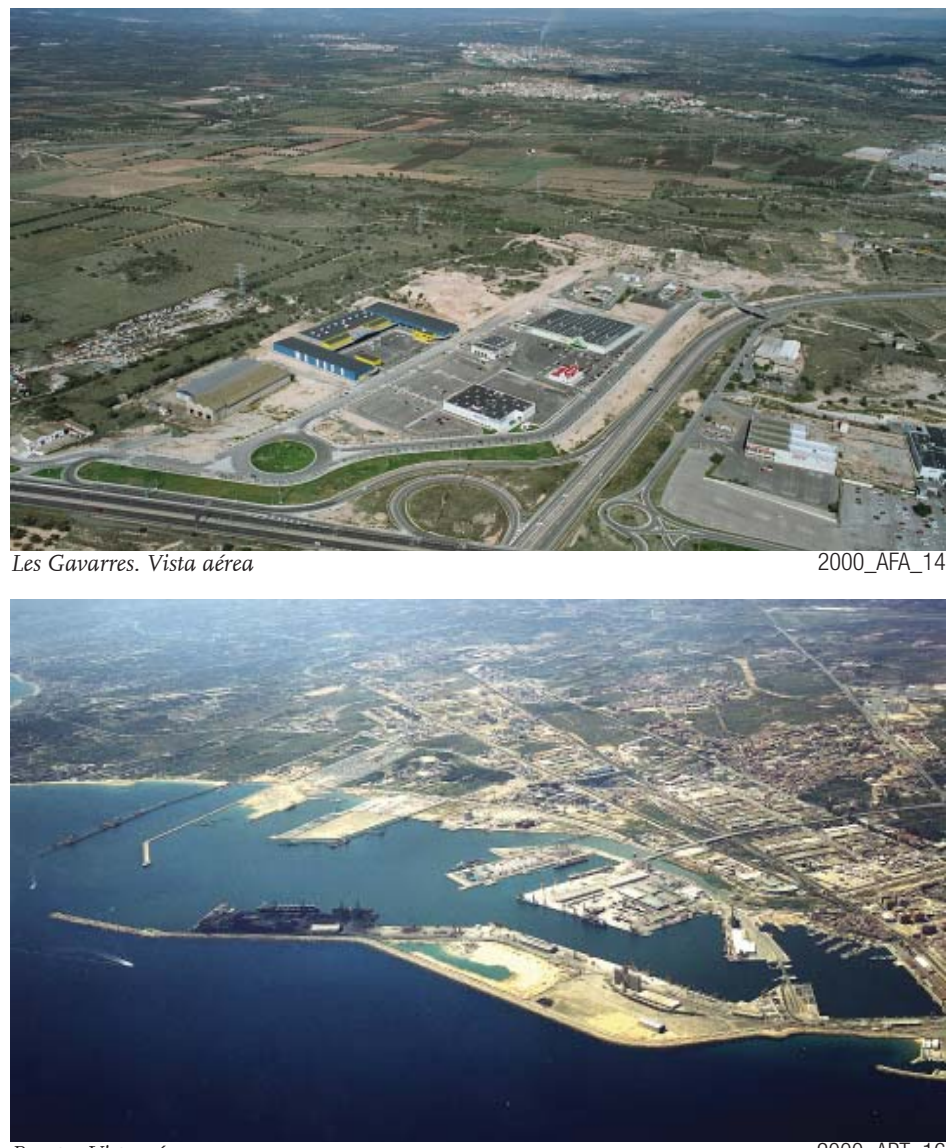

Puerto. Vista aérea

2000_APT_16
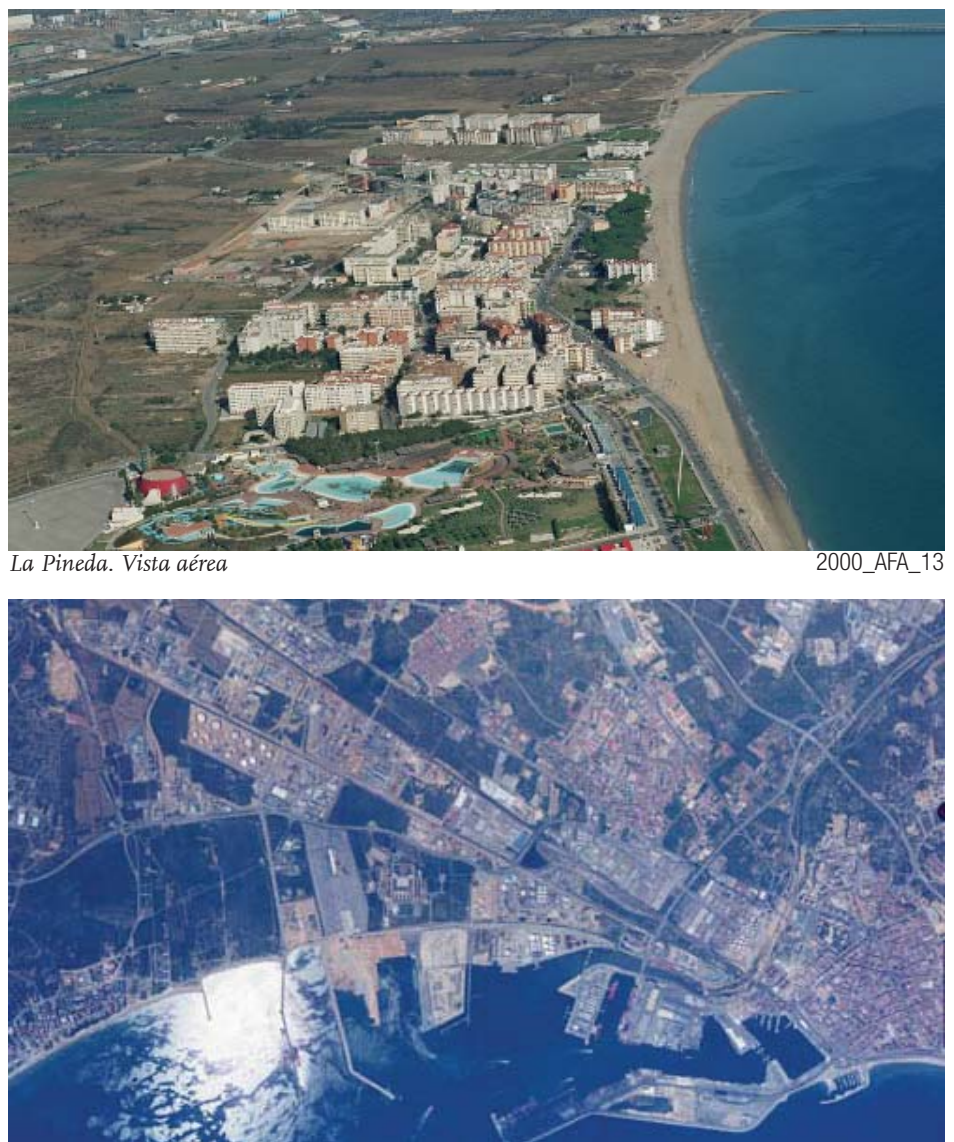

Tarrgona. Vista aérea

2000_APT_15
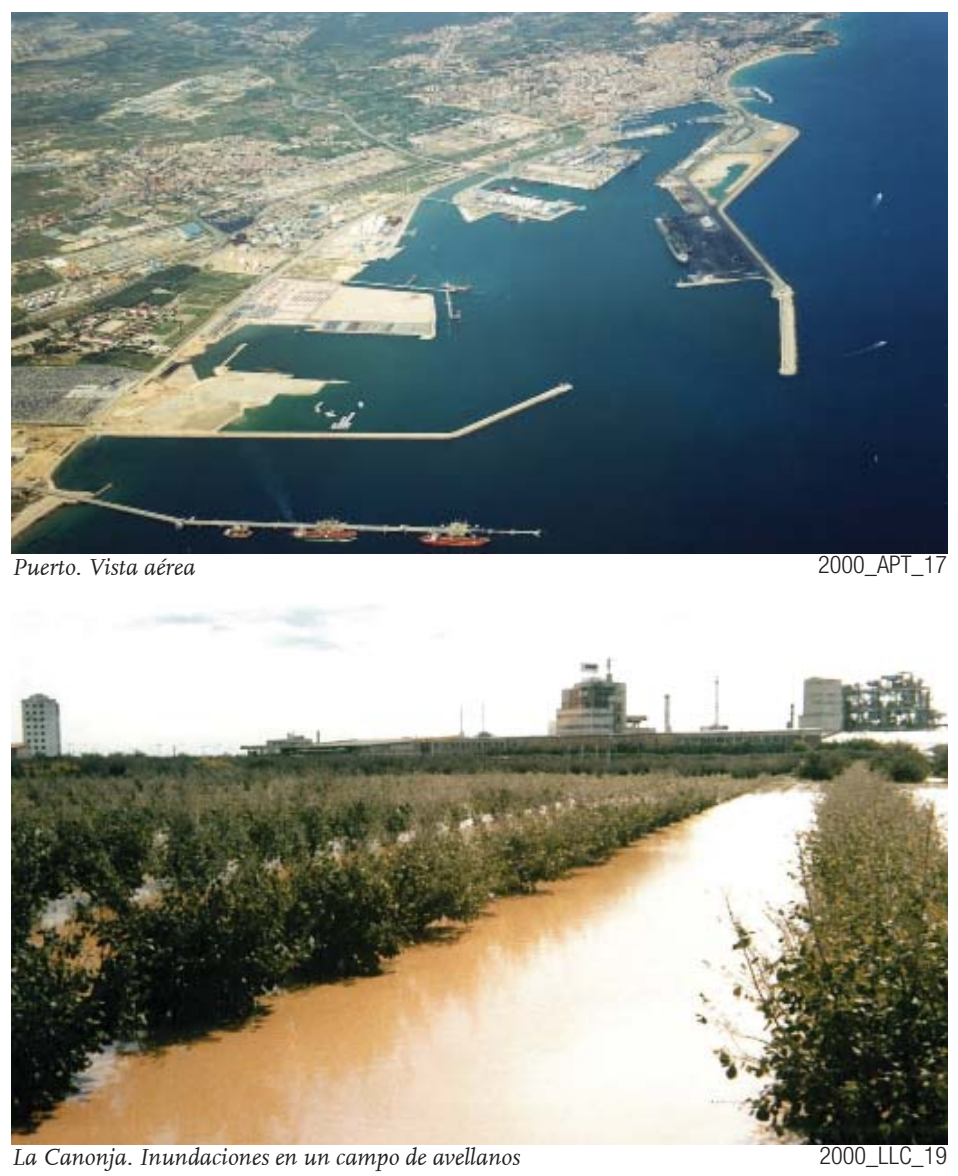

La Canonja. Vista de los alrededores del pueblo

218 


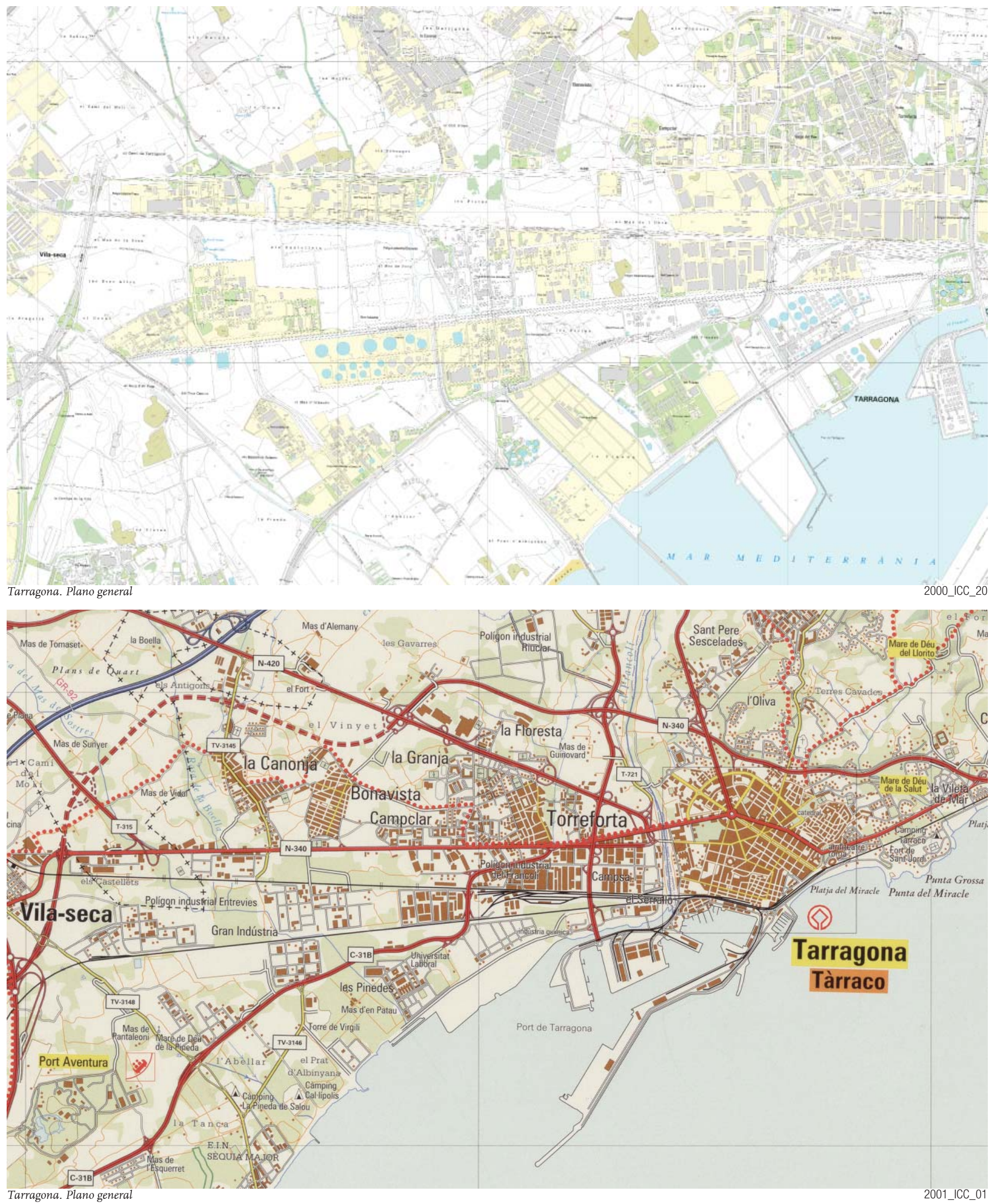




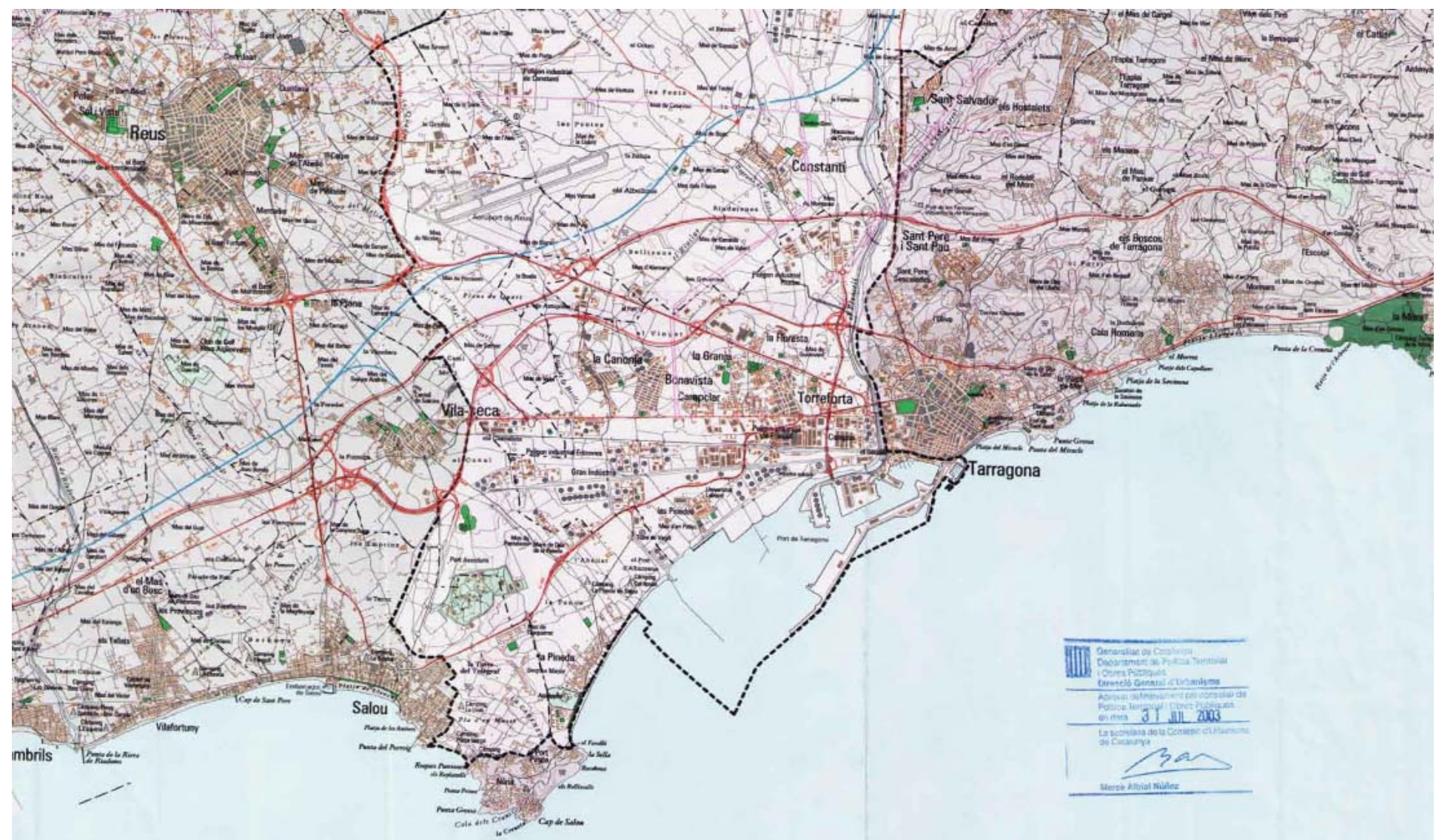

PDUAITCT. Situación

2003_PDUAI_01

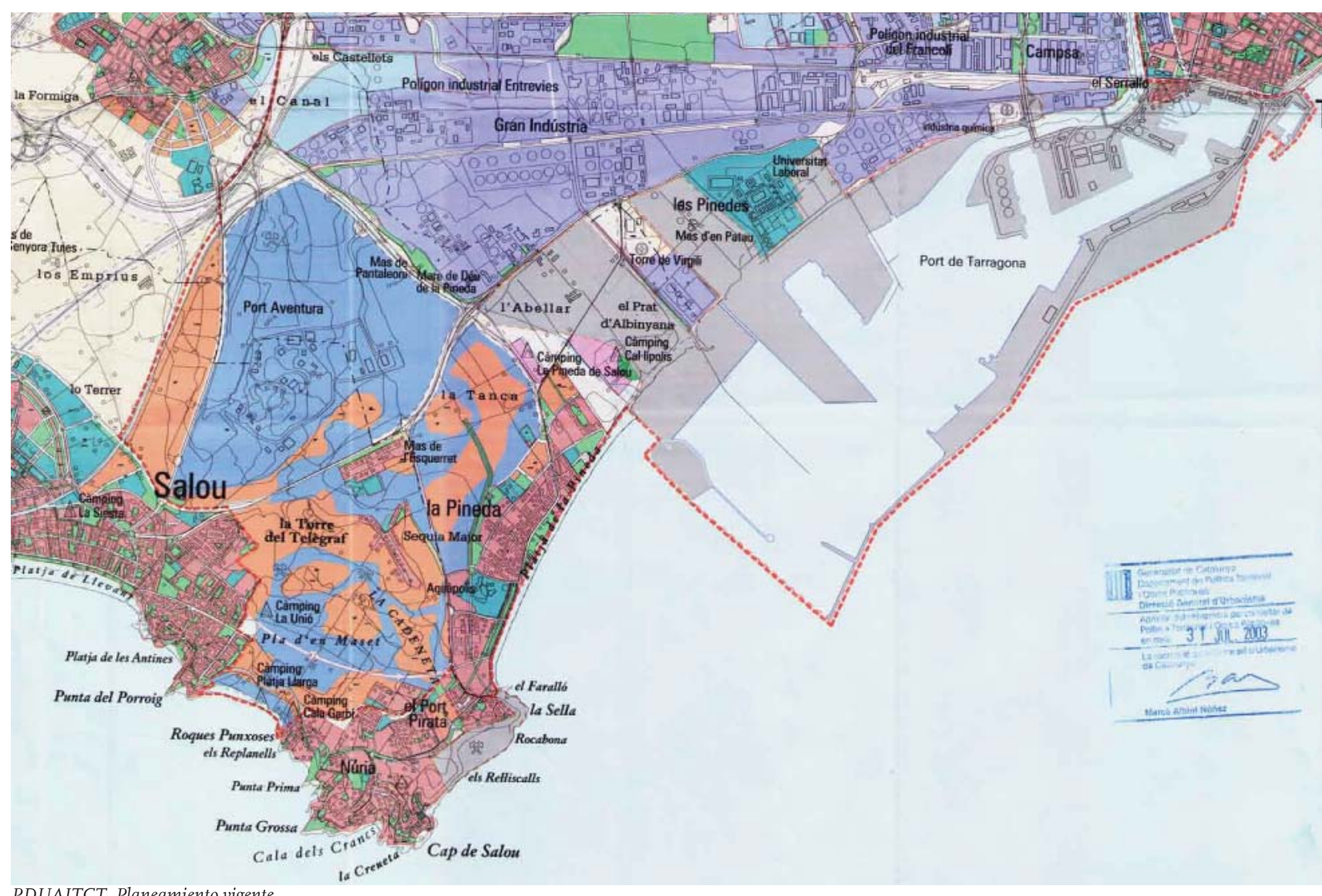




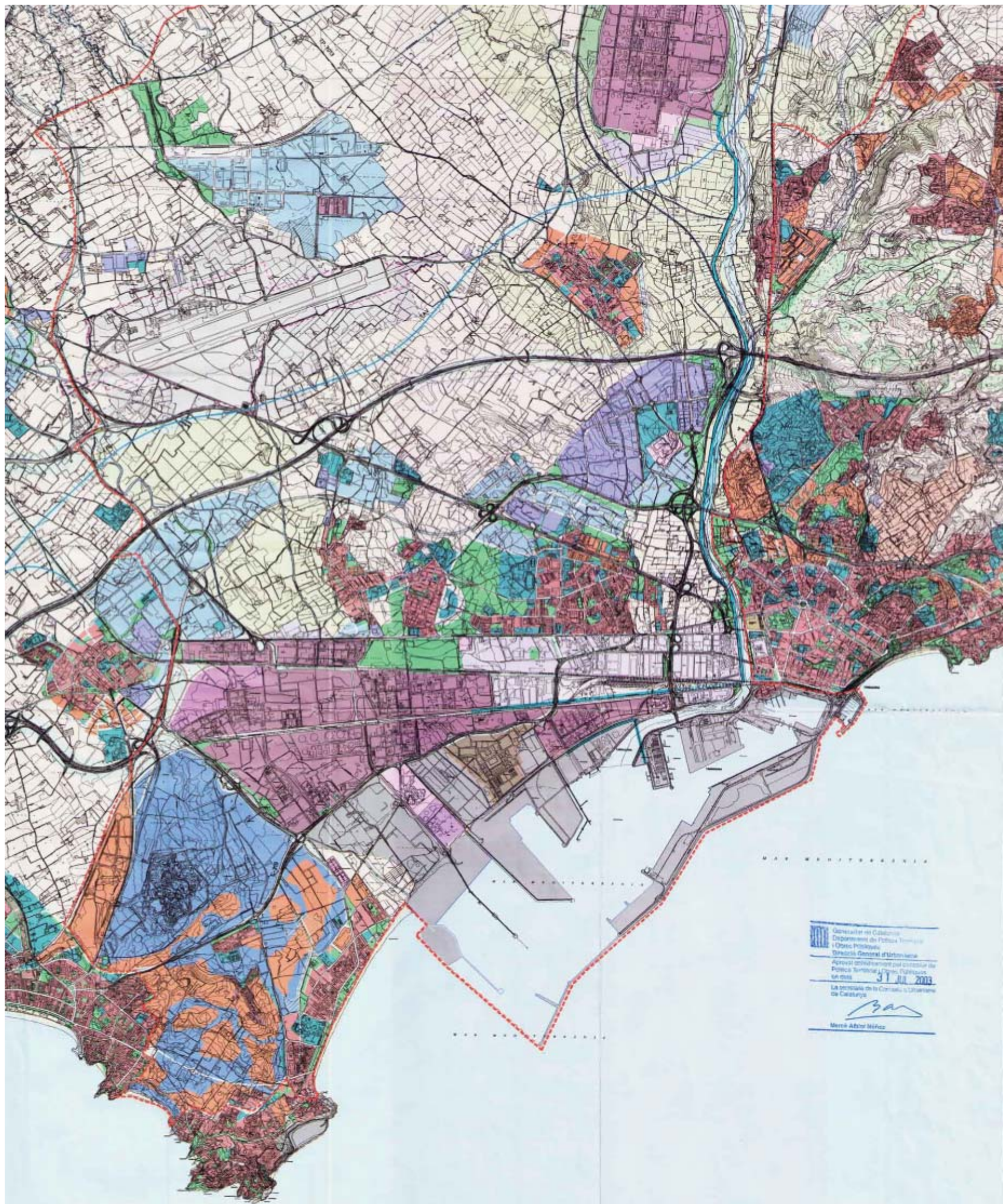



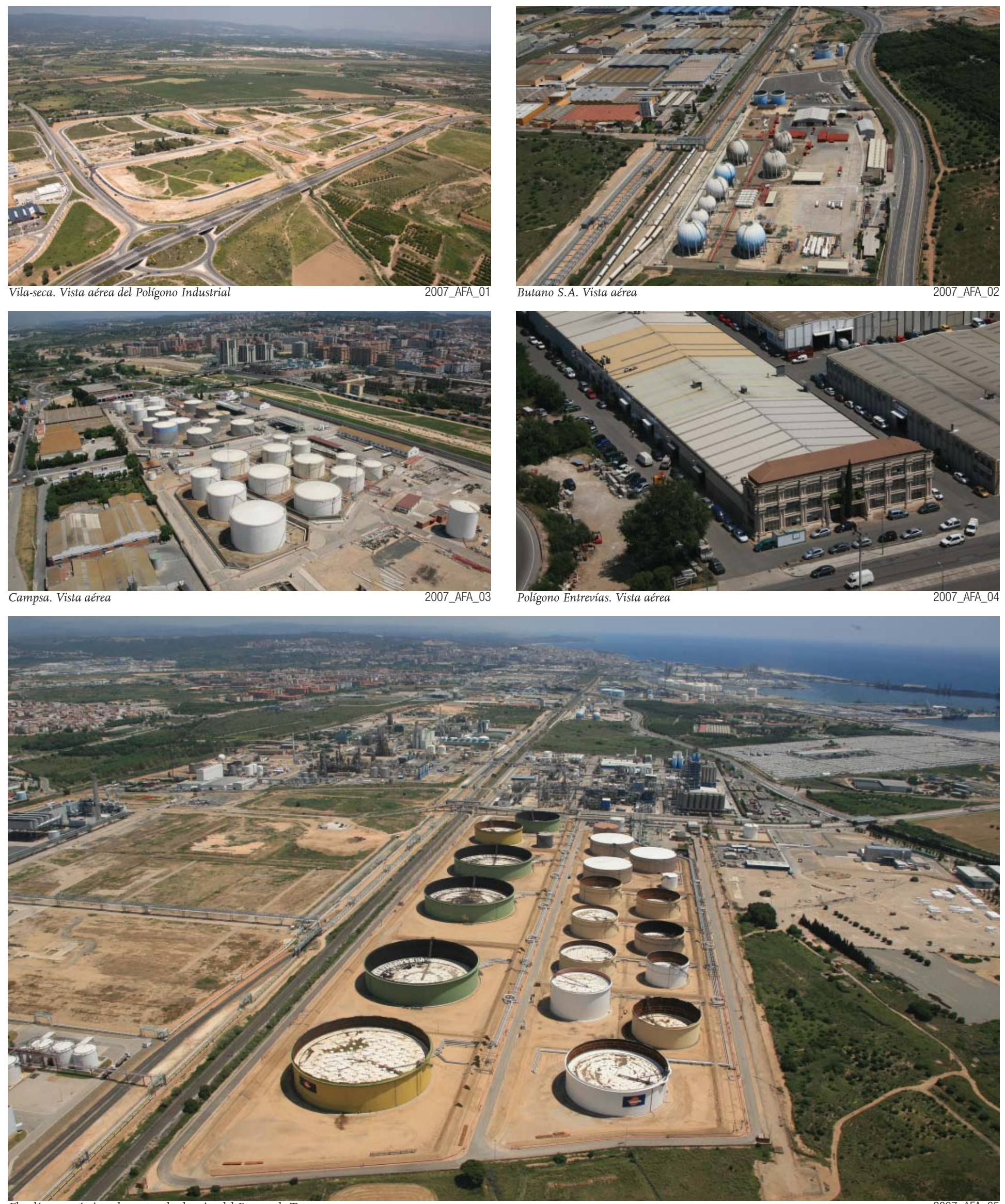

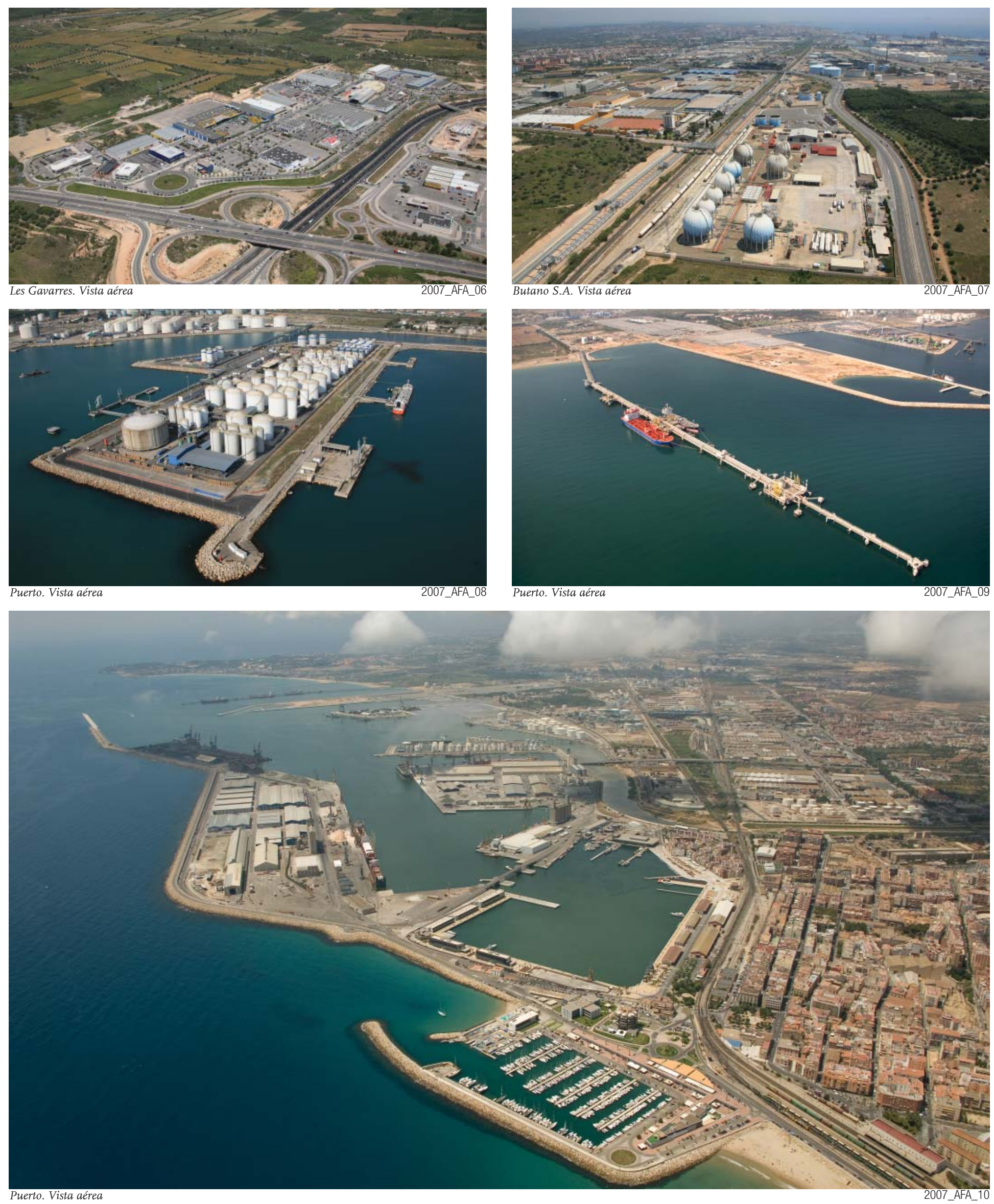


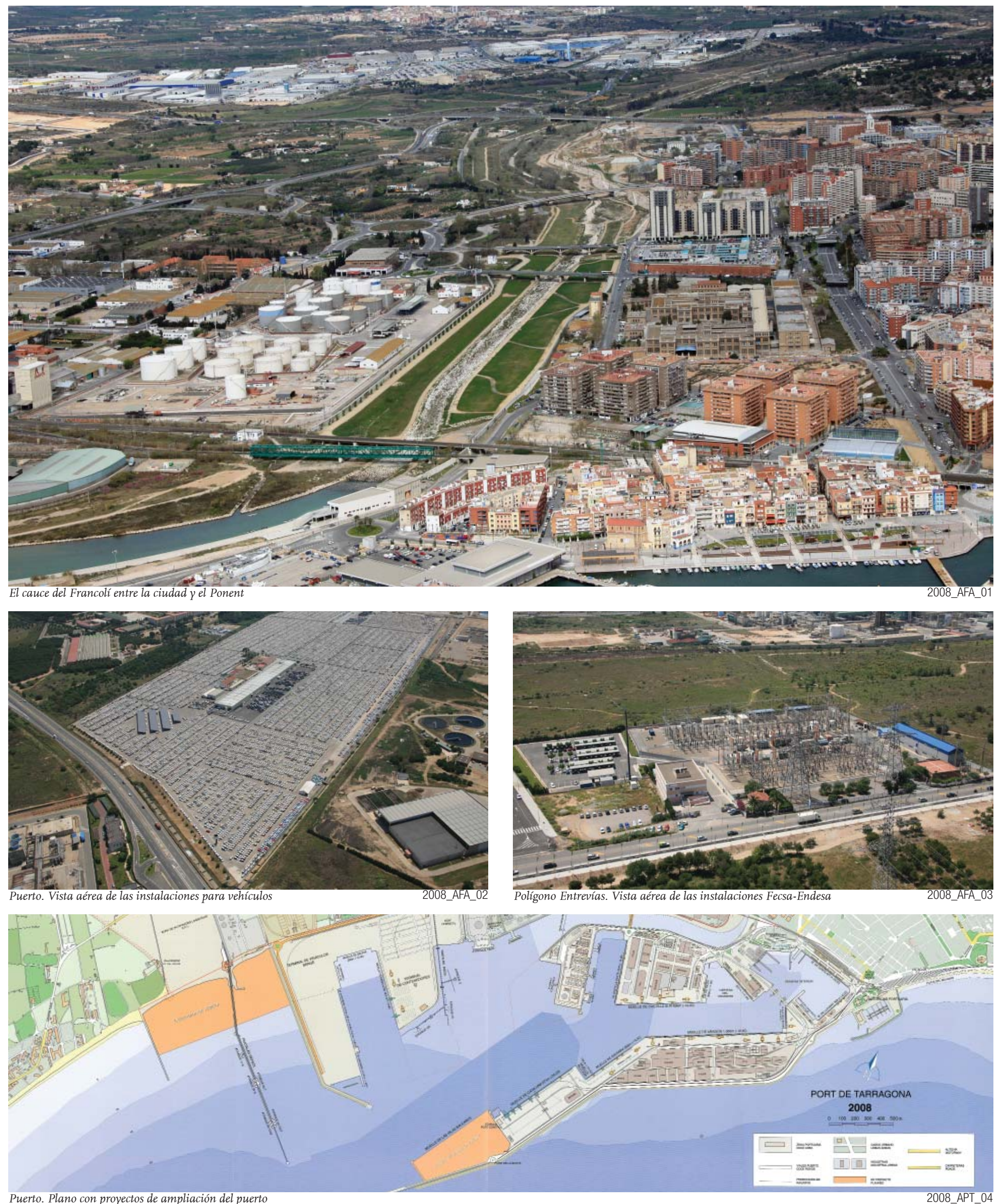




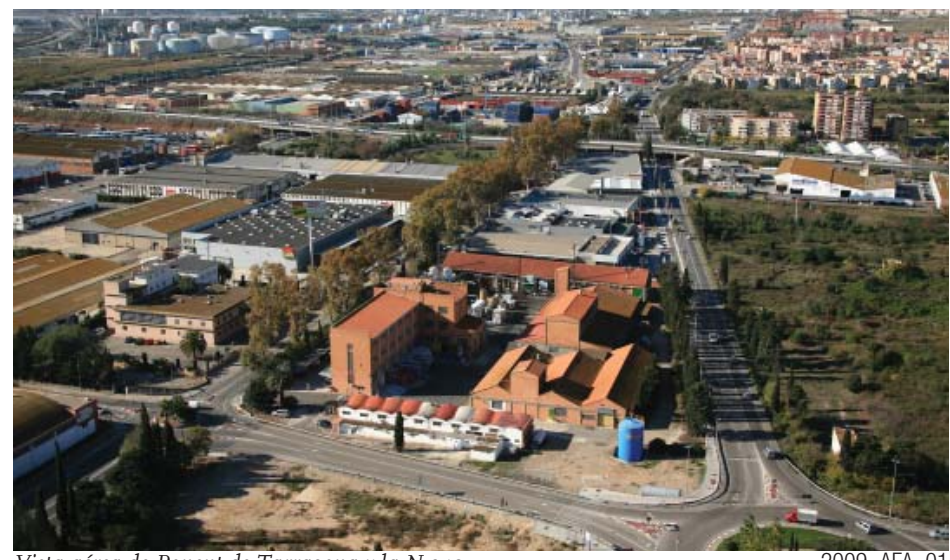

Vista aérea de Ponent de Tarragona y la N-340

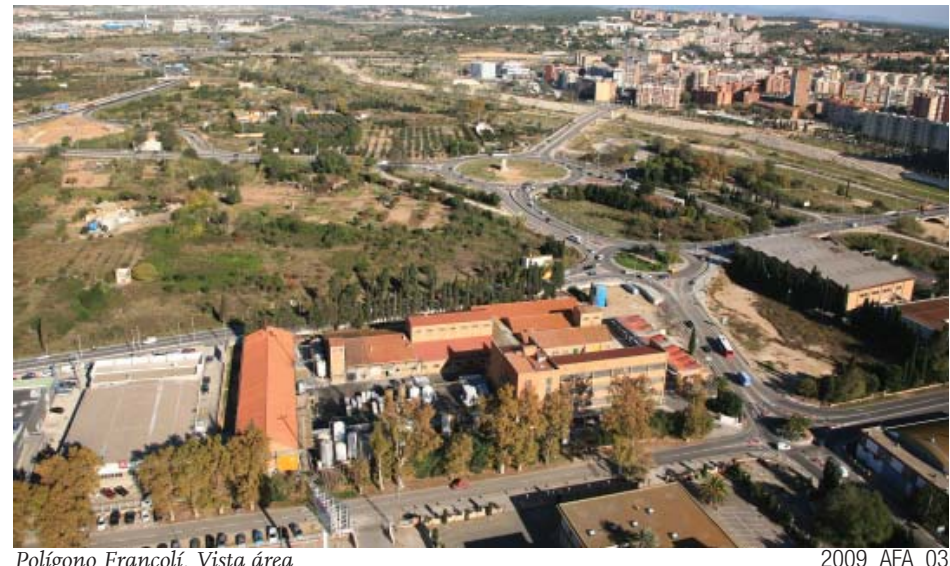
Polígono Francolí. Vista área
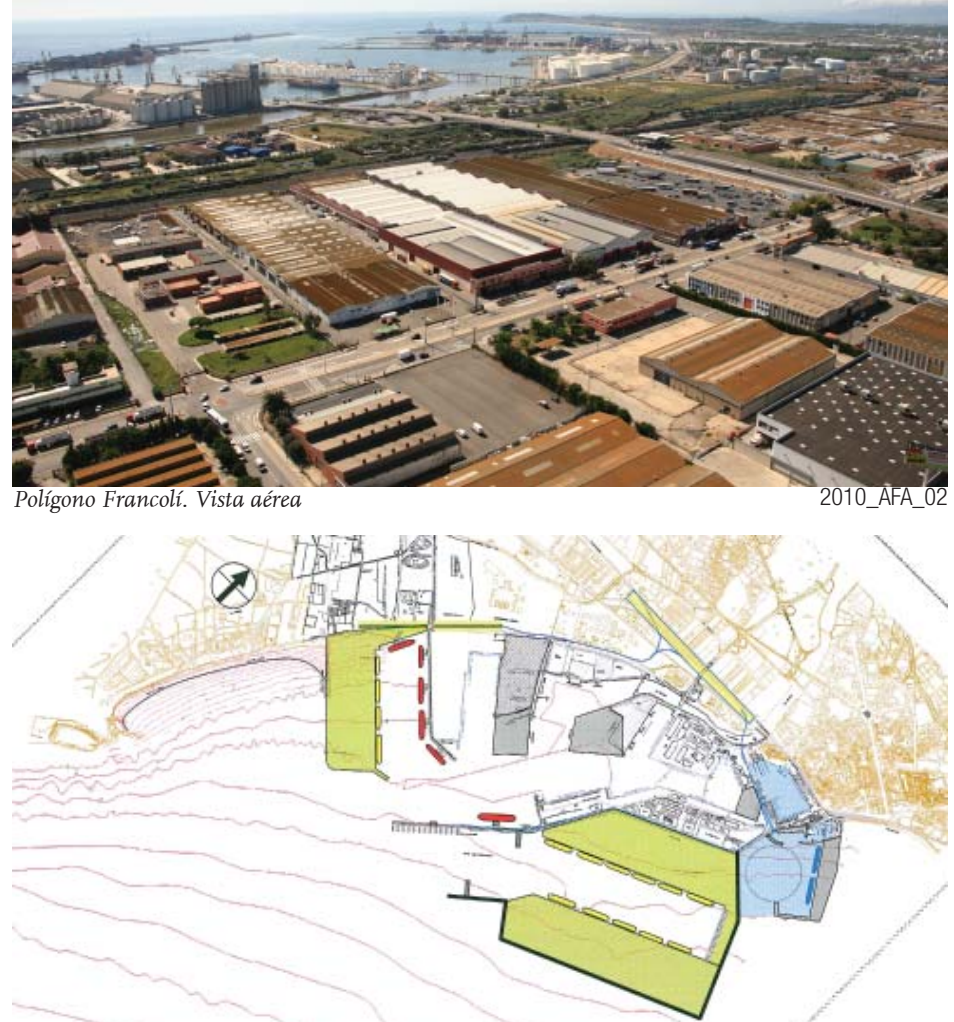

Puerto. Plan Director

2010 APT 03
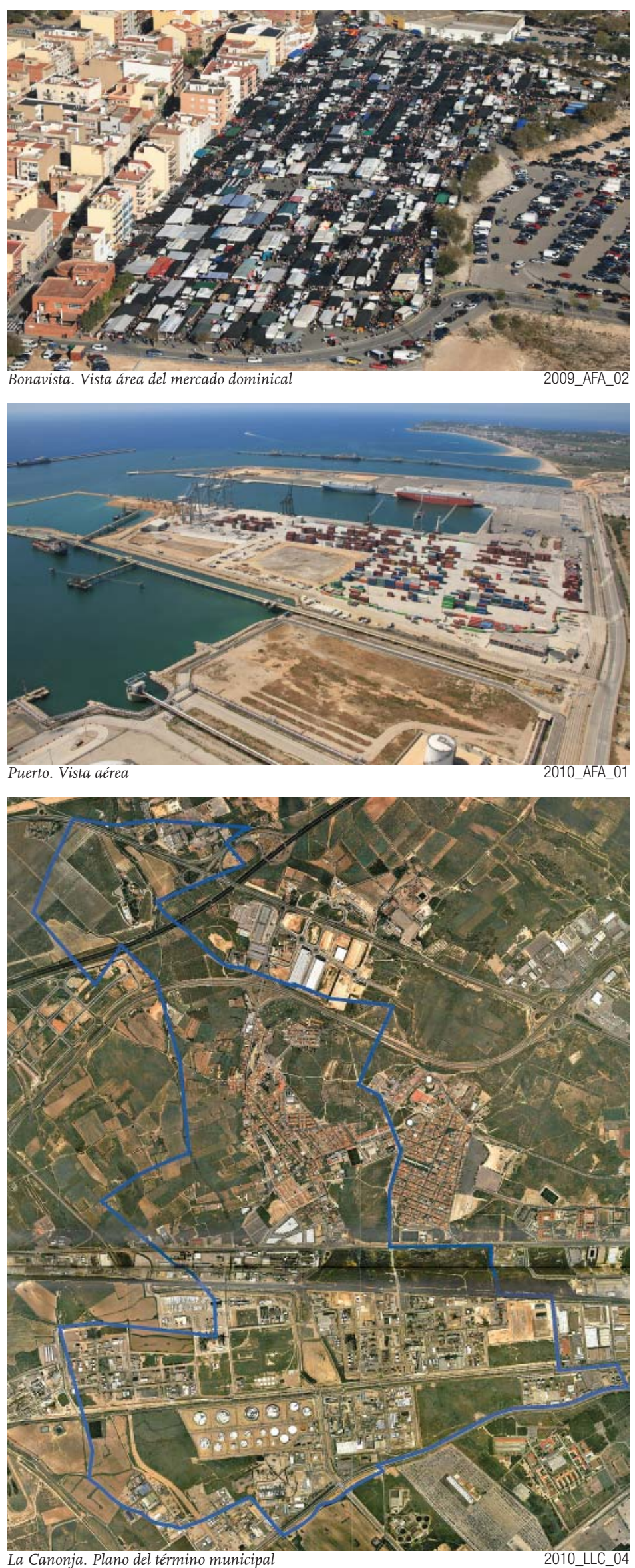


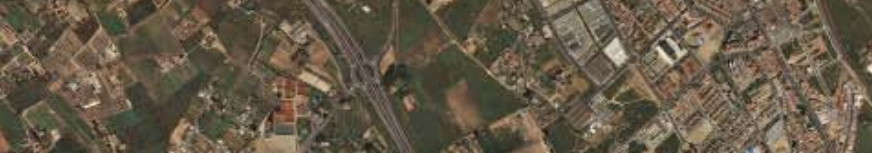

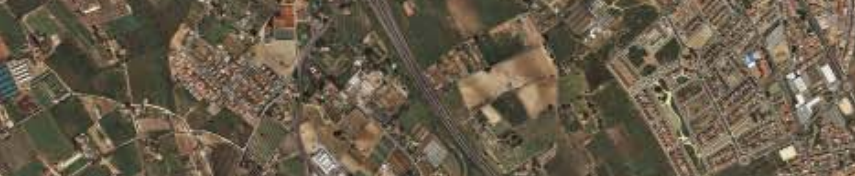

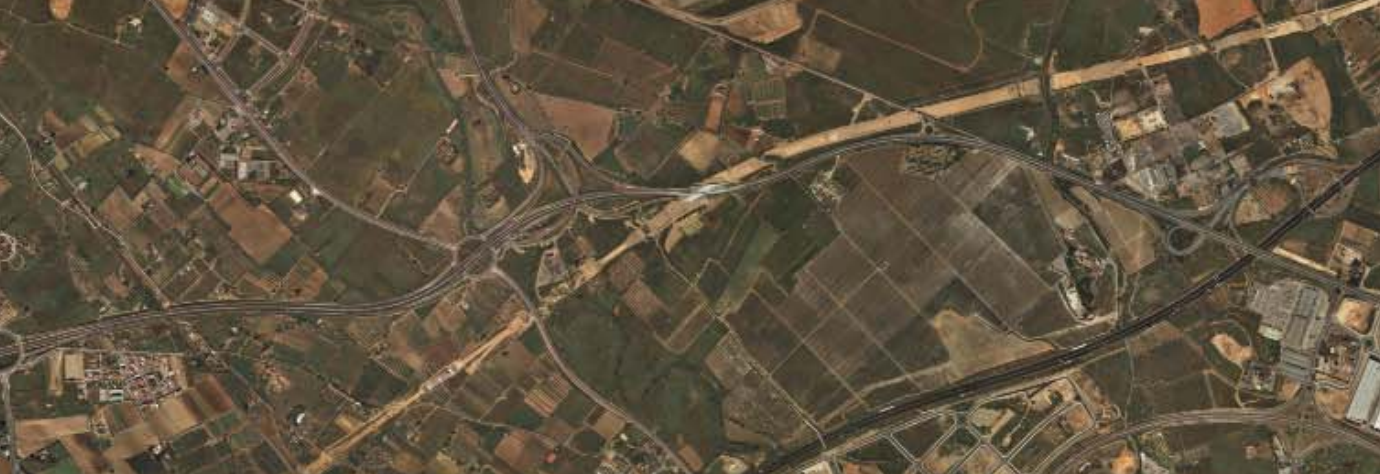

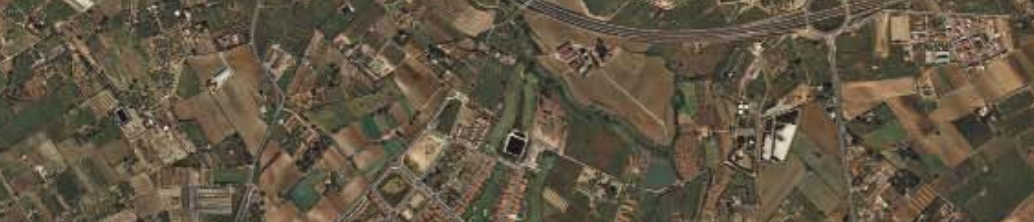
2.0. - 20

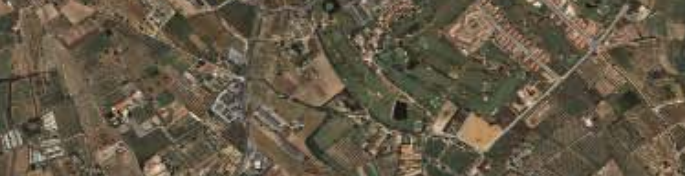

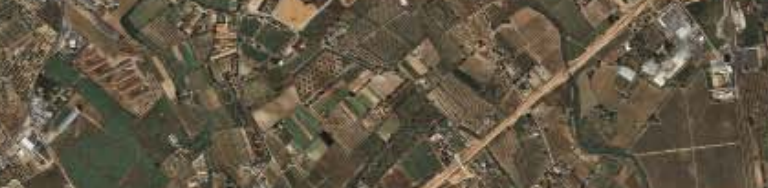

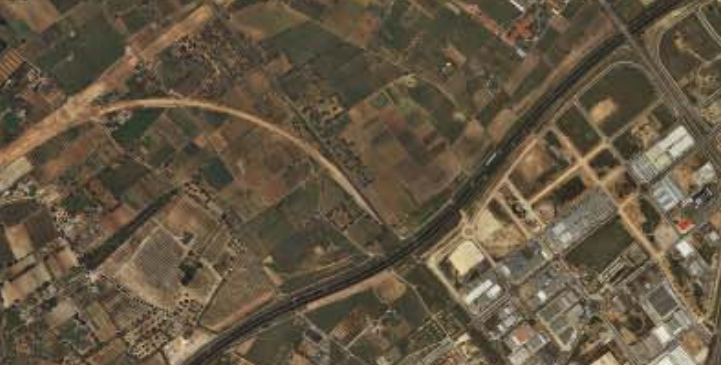
2

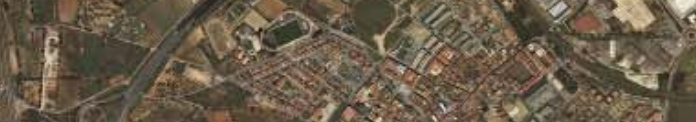

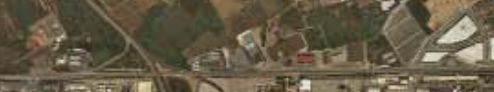

(5) (⿻) (5)

$+2 x^{2}=0$

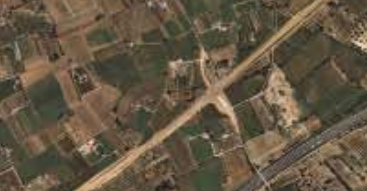

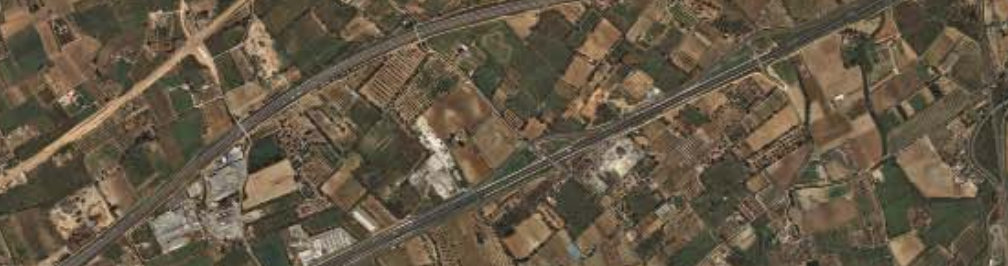
$=\log ^{2}+2$

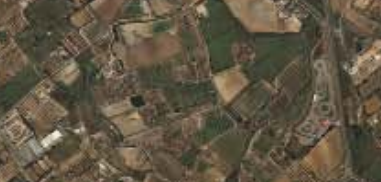

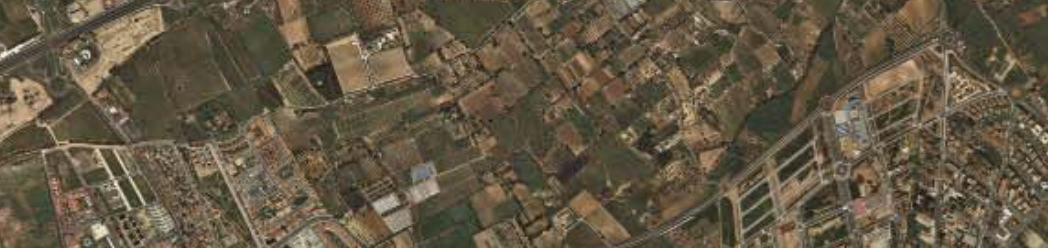

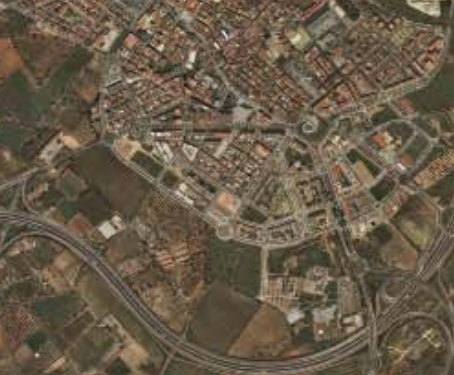

15 test

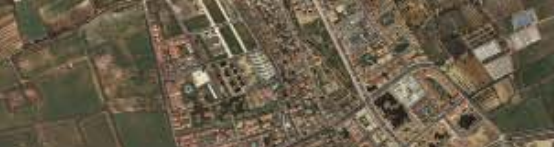

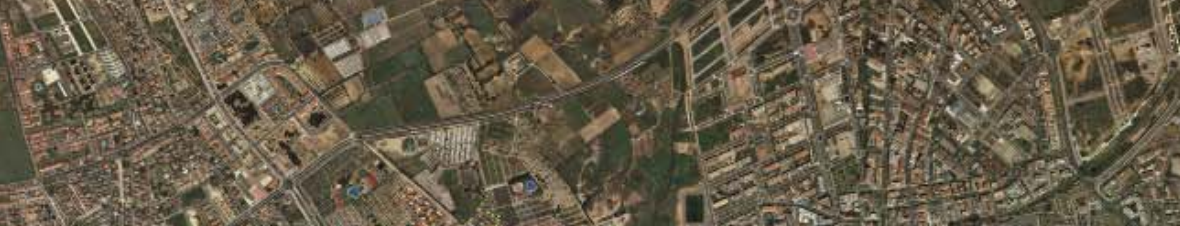

$\int^{2}$ XXin ) ini 


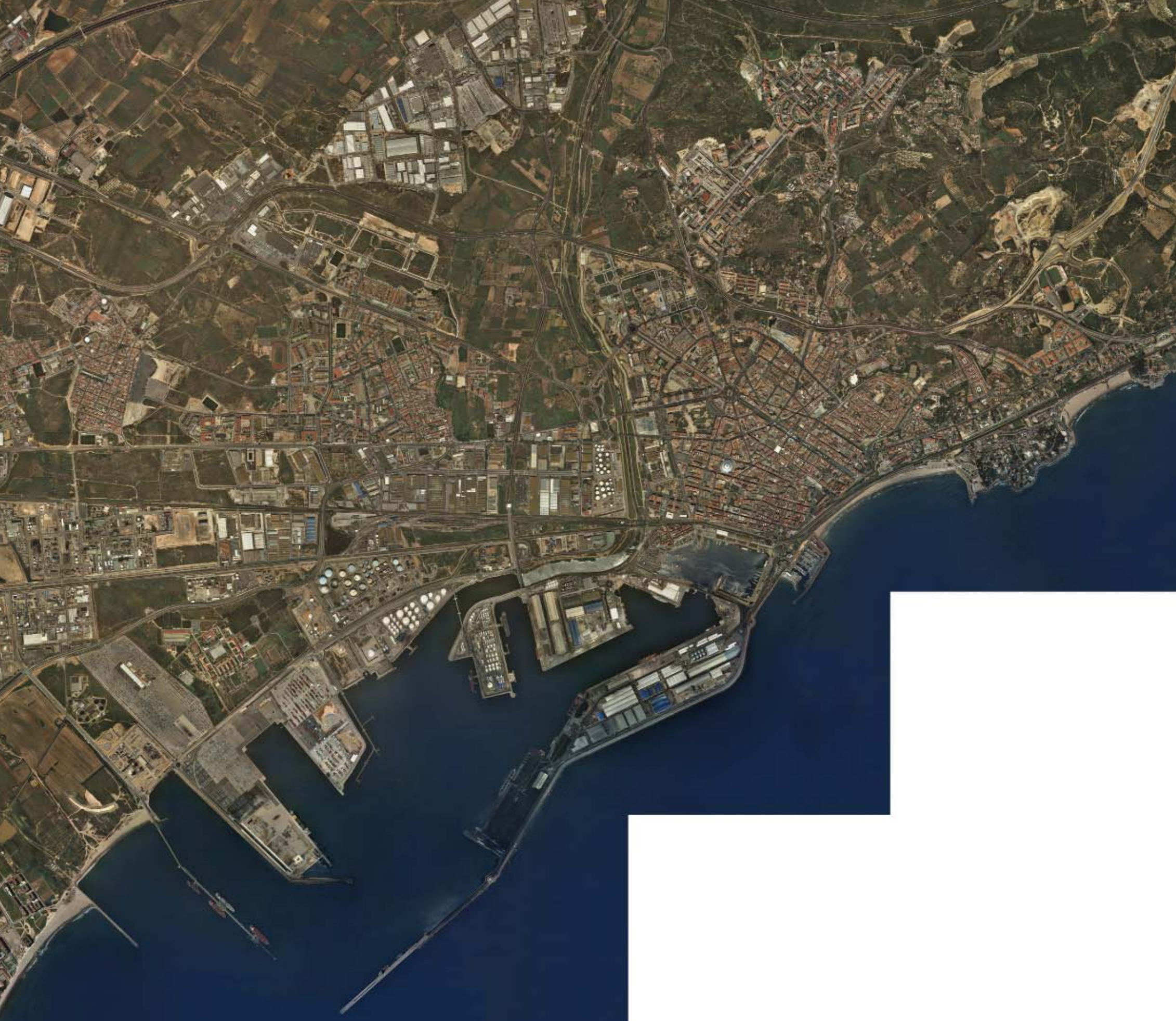

PLANEAMIENTO URBANÍSTICO EN CONTROVERSIA.

"La creciente distancia entre la situación de hecho en el territorio urbano europeo y la ciudad dibujada, o si se prefiere, entre el urbanismo real y la urbanística formal, está poniendo en tela de juicio la utilidad de la planificación urbanística como instrumento principal para la previsión y el control de las transformaciones territoriales en curso. Este cuestionamiento que ha llegado también a determinados círculos académicos en los que ha empezado a hablarse de la "crisis del planeamiento", se está produciendo tanto en aquellos países en los que la configuración legal y administrativa del planeamiento urbanístico responde al tradicional modelo del "plan-ley", como en los del "plan-concertación".

Ciertamente no podemos ignorar las progresivas dificultades que está encontrando la planificación urbanística tradicional para abordar los nuevos problemas que plantea la nueva etapa del proceso de urbanización en el que estamos inmersos. Sin embargo tampoco podemos olvidar su gran utilidad y eficiencia sociales, al menos en etapas recientes del proceso de construcción de nuestras ciudades y de estructuración de nuestros territorios.
[...] Tras la etapa dorada para la planificación urbana del inicio de los años ochenta en España, con la restauración democrática en los gobiernos autonómicos y municipales, la situación posterior ha sido en general la de la normalización del planeamiento y de la gestión urbana y quizás la de su progresiva burocratización.

Las sucesivas leyes estatales y autonómicas o regionales en materia de urbanismo han tratado de encontrar, aparentemente sin demasiado éxito, respuestas adecuadas a los nuevos problemas. Situación que entendemos puede hacerse extensiva, no sin importantes matices, a los países de nuestro entorno, en los que una nueva reforma urbanística es todavía un reto pendiente y un tema de enorme actualidad."

Extracto de la introducción escrita por Antonio Font Catedrático de Urbanística, al libro Planeamiento urbanístico. De la controversia a la renovación. Barcelona: Diputación de Barcelona, 2003. 2010 ICC 05 


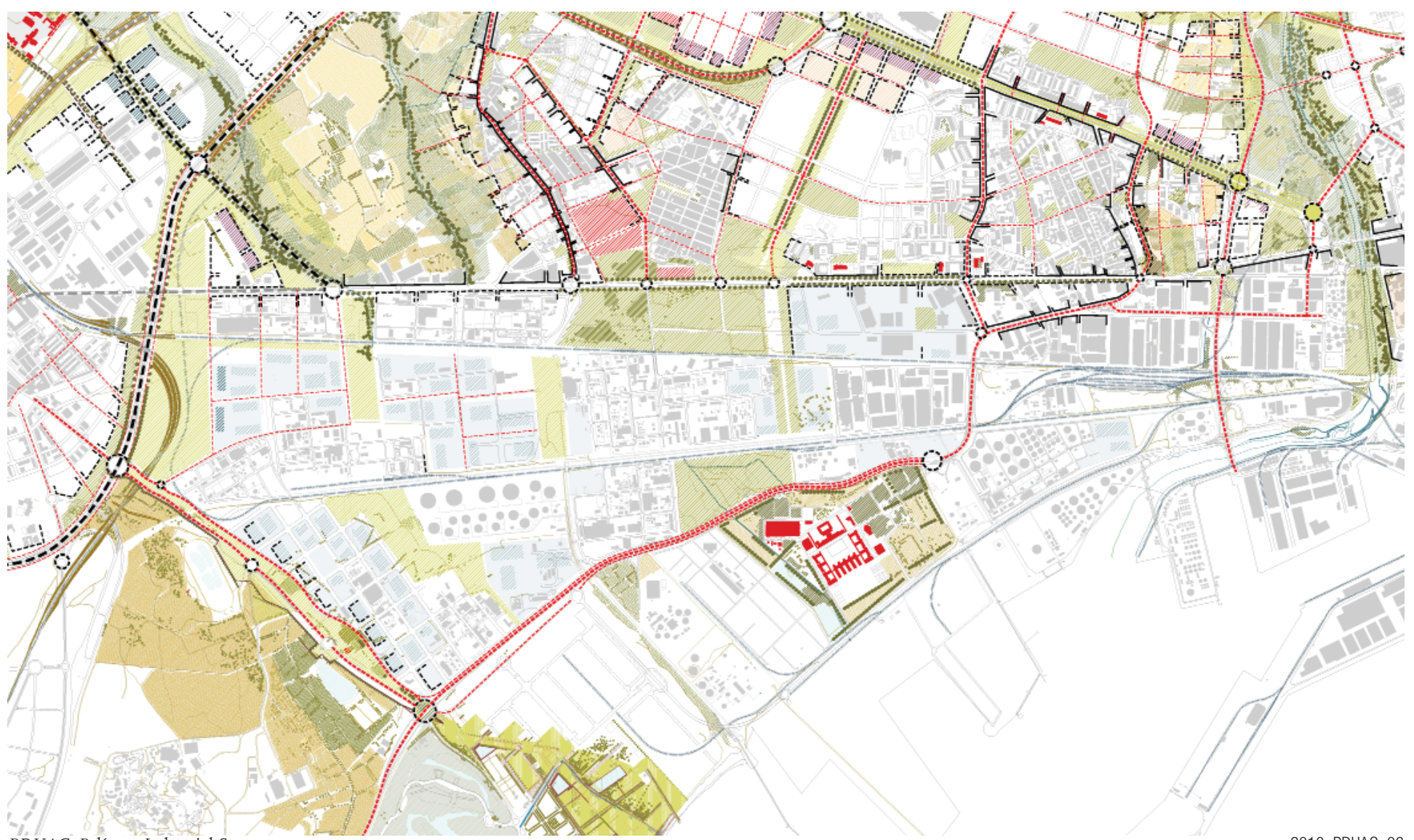

PDUAC. Polígono Industrial Sur
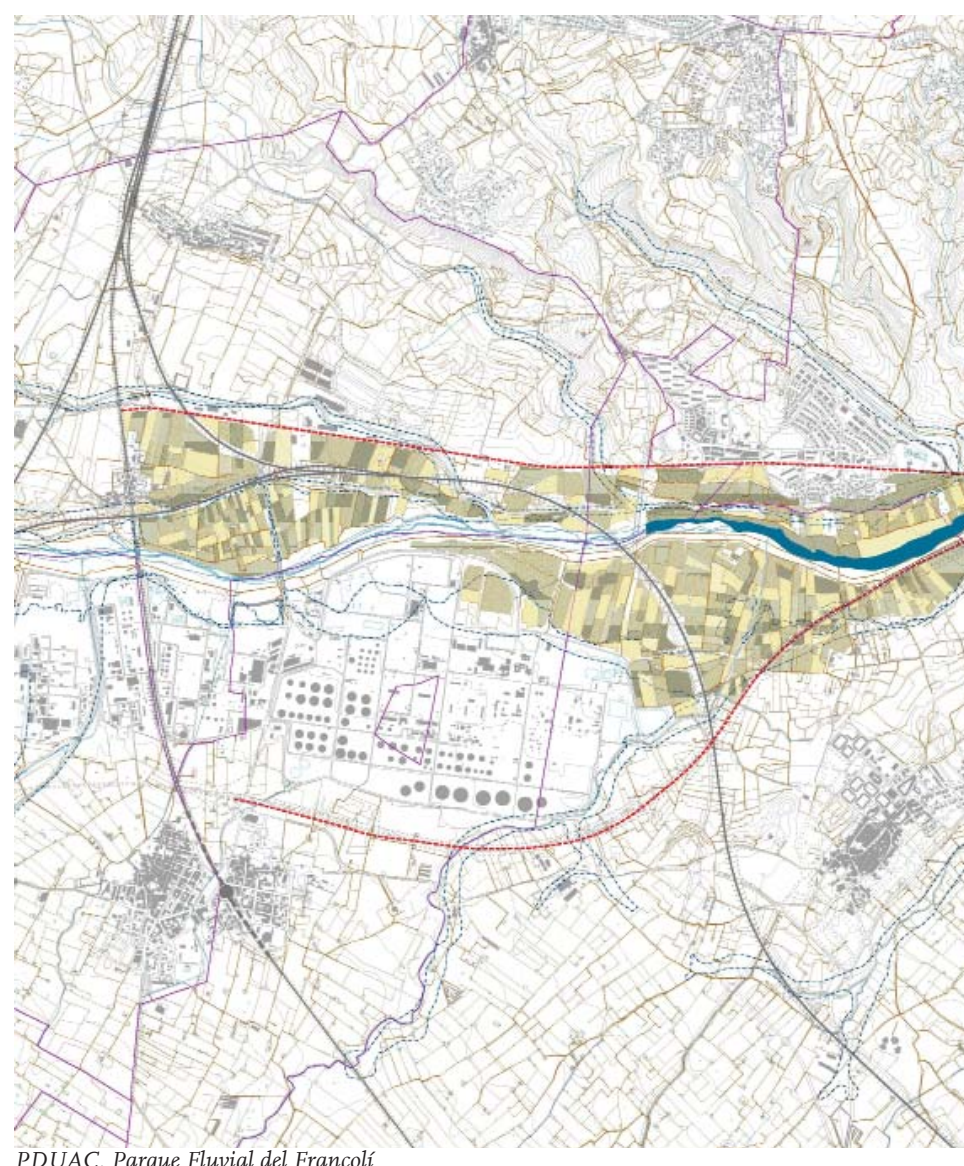

2010_PDUAC_06 

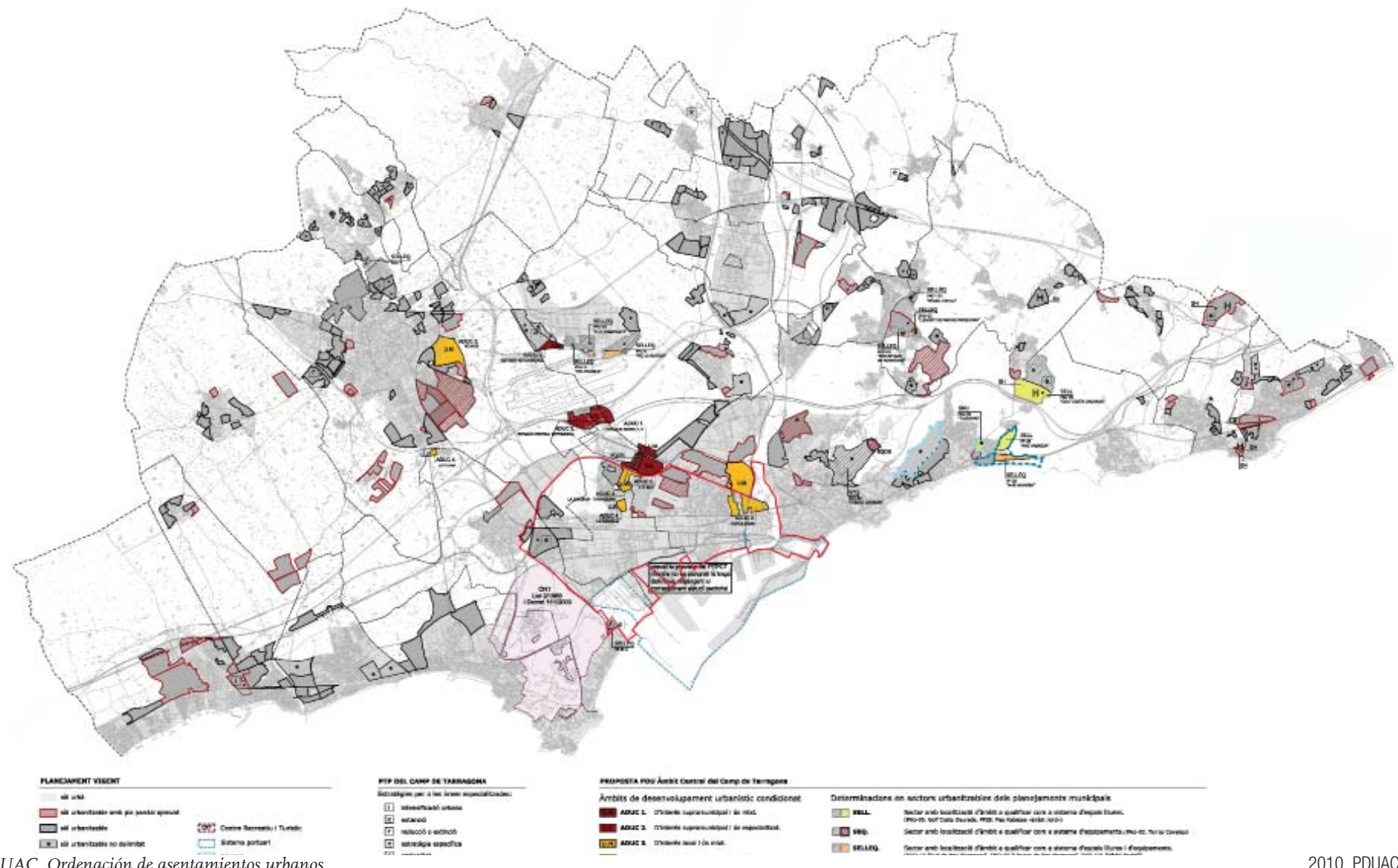

PDUAC. Ordenación de asentamientos urbanos

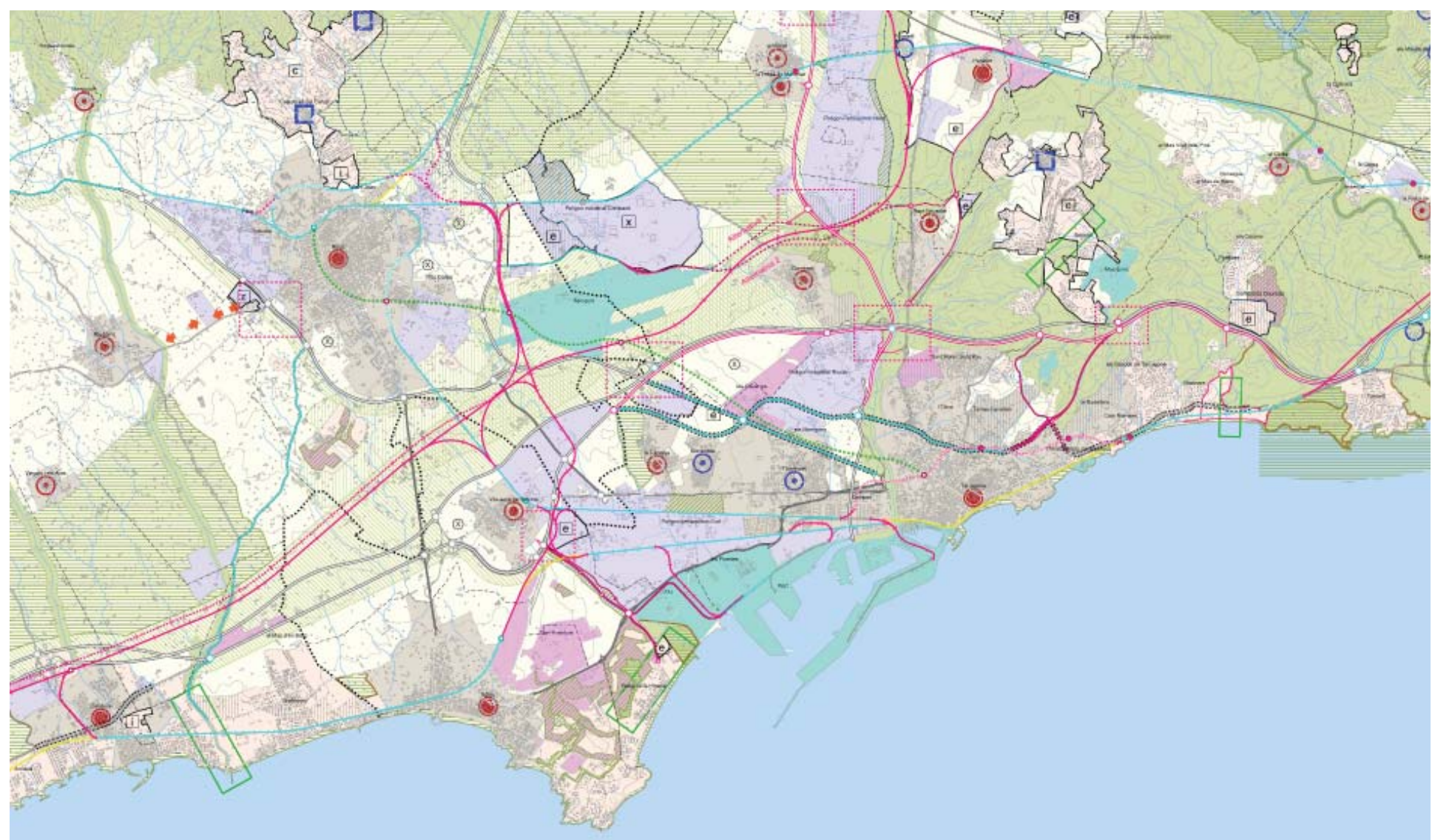



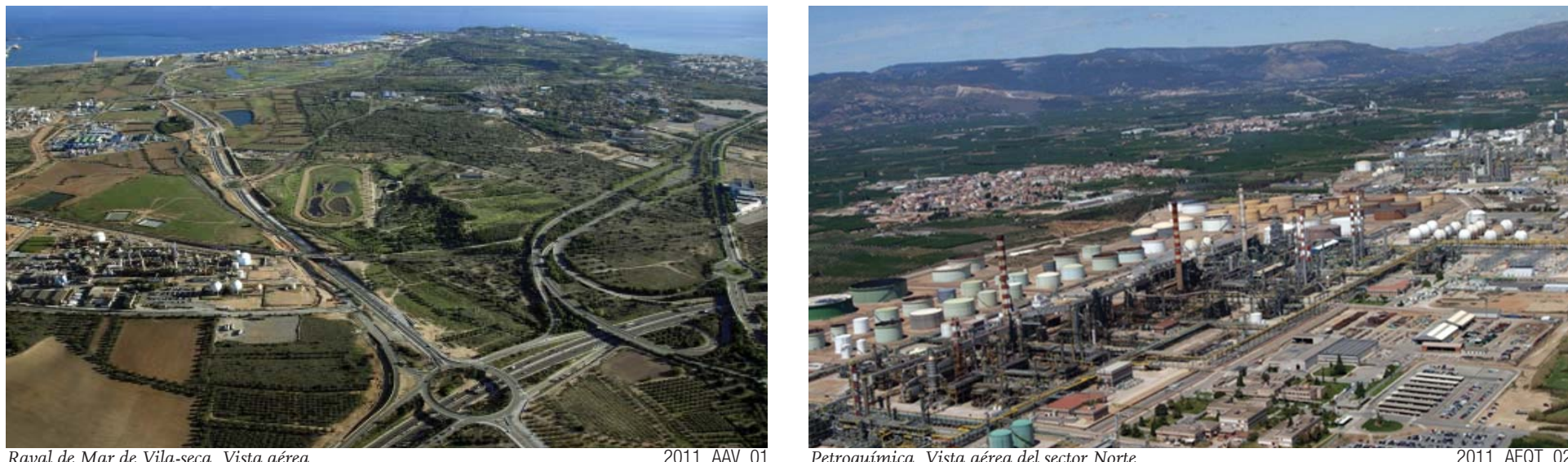

Raval de Mar de Vila-seca. Vista aérea.

2011 AAV 01
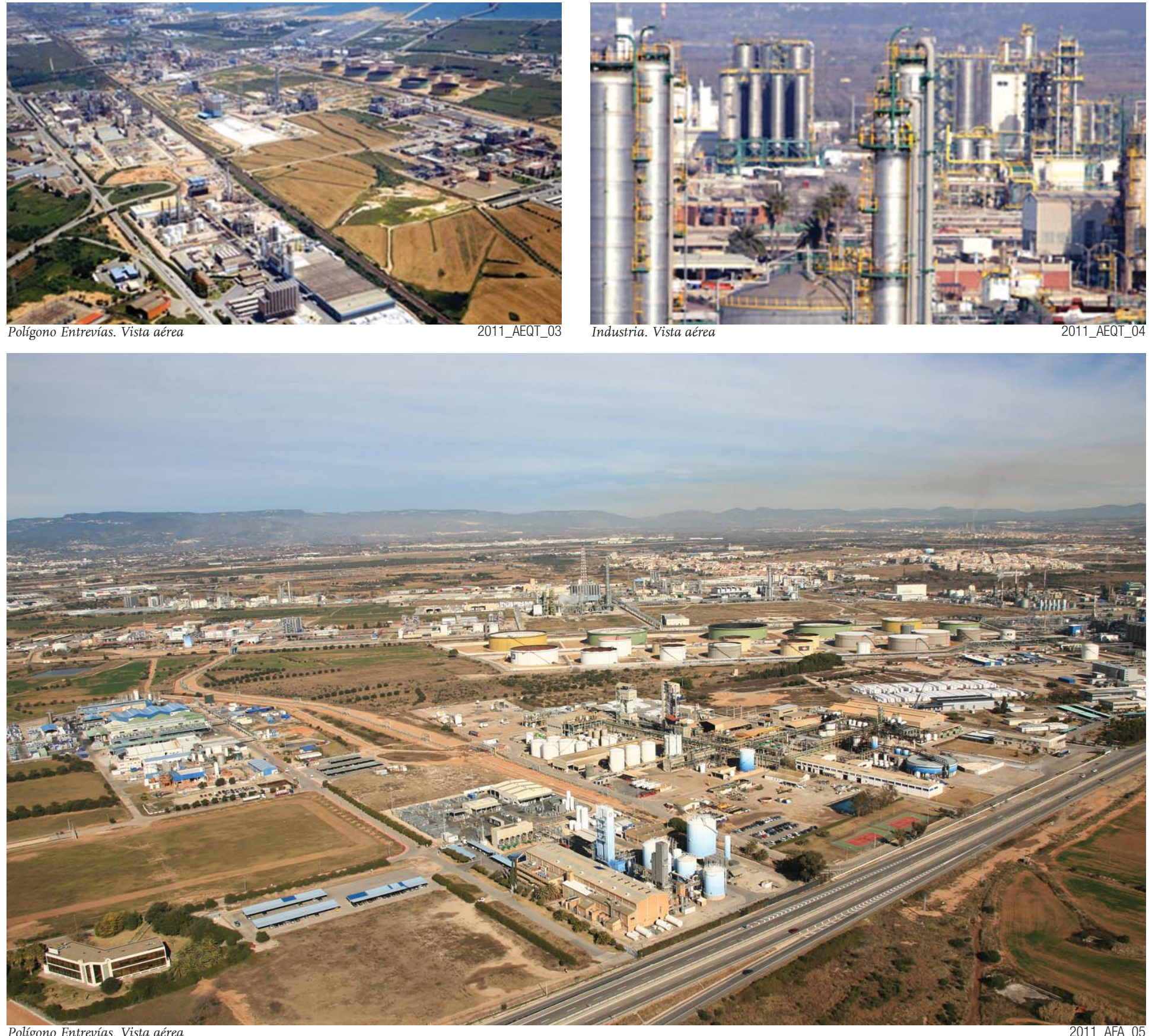

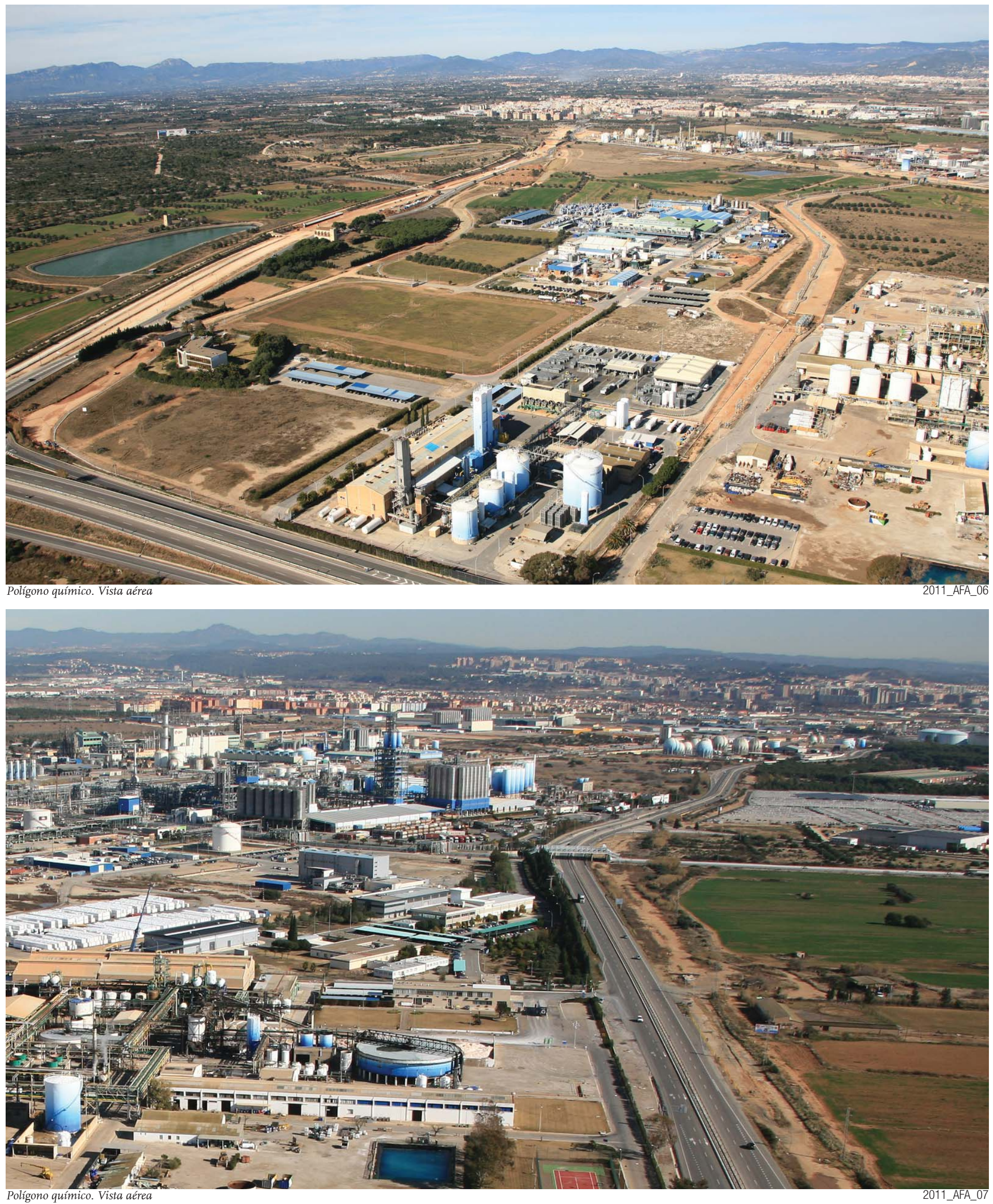


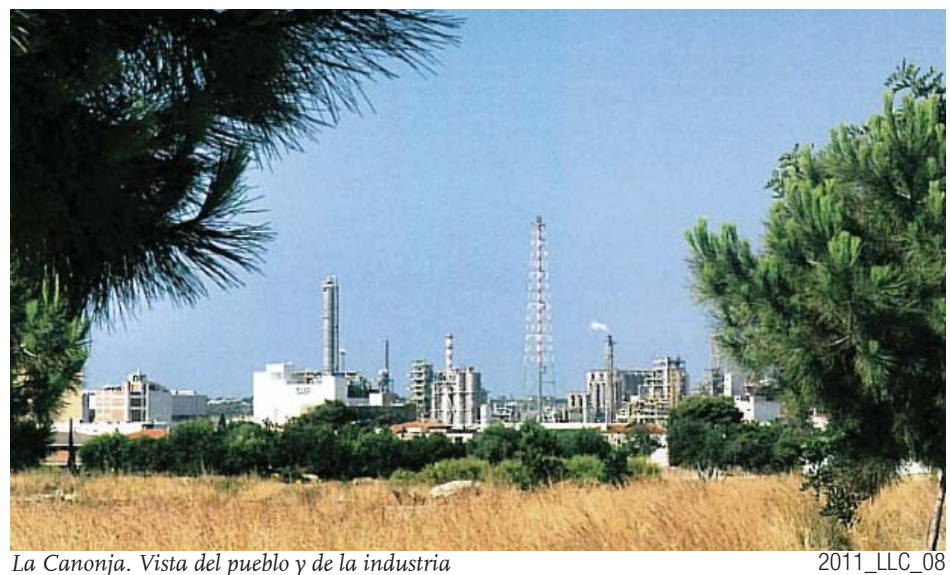

La Canonja. Vista del pueblo $y$ dela industria
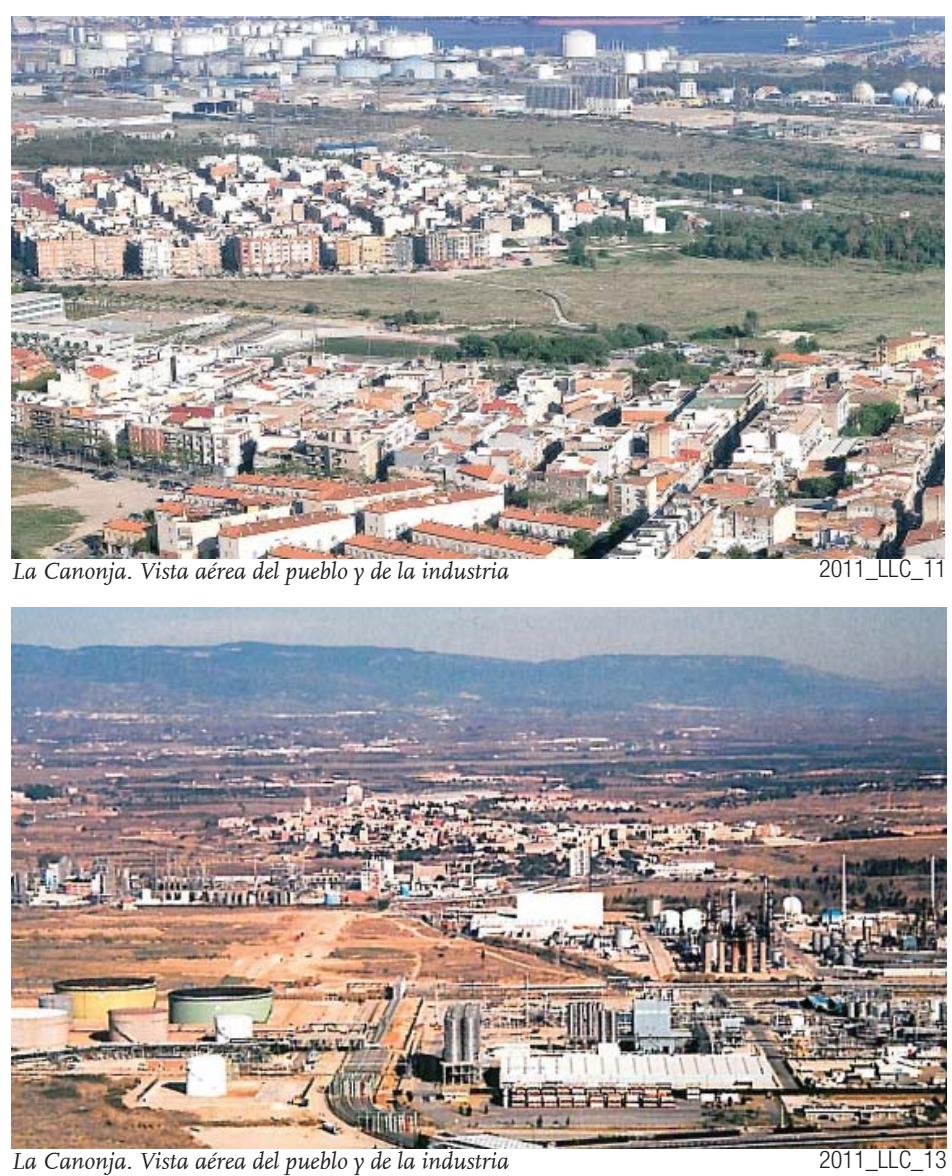
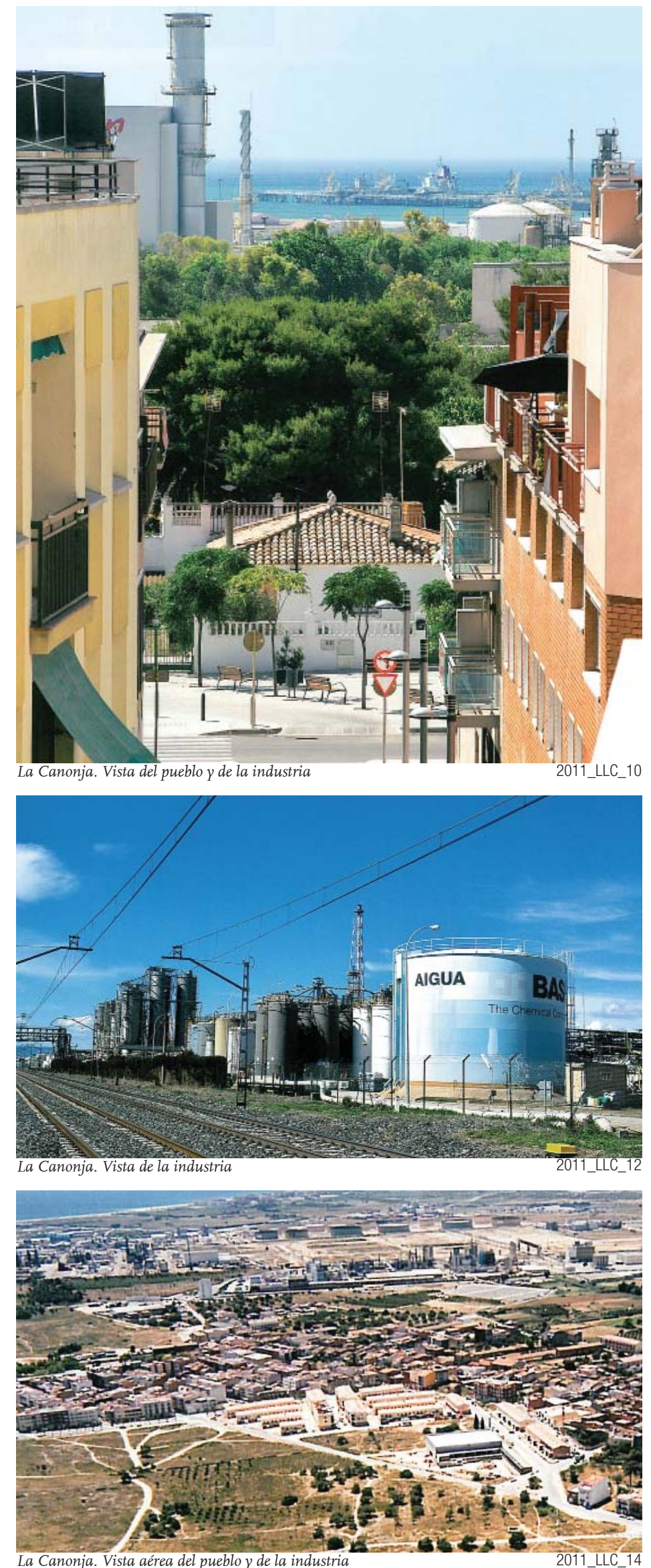


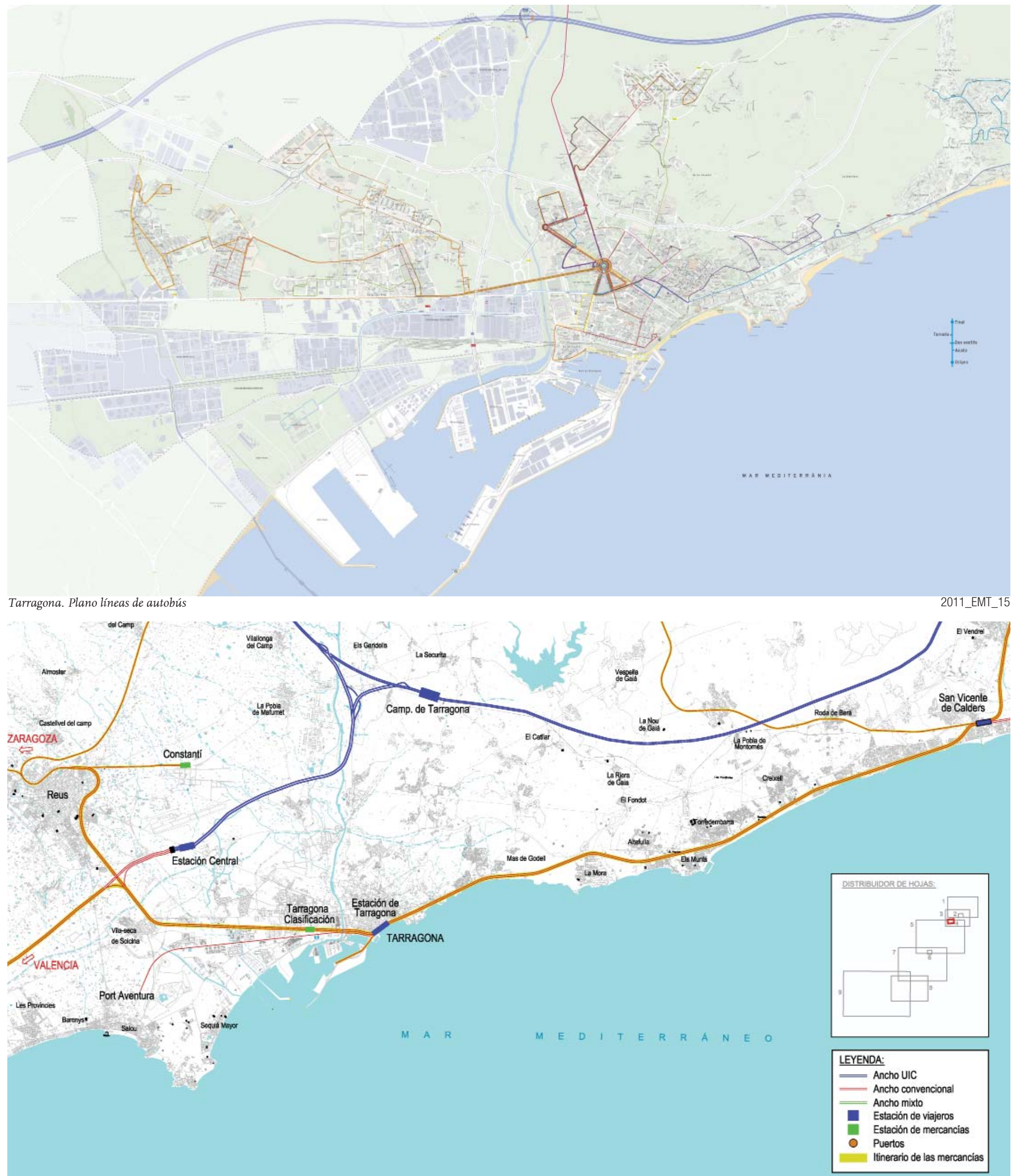


DE LA NECESIDAD DE REFLEXIONAR SOBRE EL TERRITORIO

"Una simple mirada a una fotografía aérea actualizada del núcleo central y más poblado del Camp de Tarragona hace darse cuenta, hasta a los ojos más inexpertos, del monumental desorden provocado sobre el territorio por las nuevas implantaciones industriales, residenciales e infraestructurales, la mayor parte de la cuales nacidas en la década estudiada de los años sesenta."

Extracto del libro, La industrialització de Tarragona (1957-1971)i les seves circumstàncies. J. Llop Tous, Tarragona: Arola, 2002.

\section{REPORTAJE FOTOGRÁFICO ACTUAL}
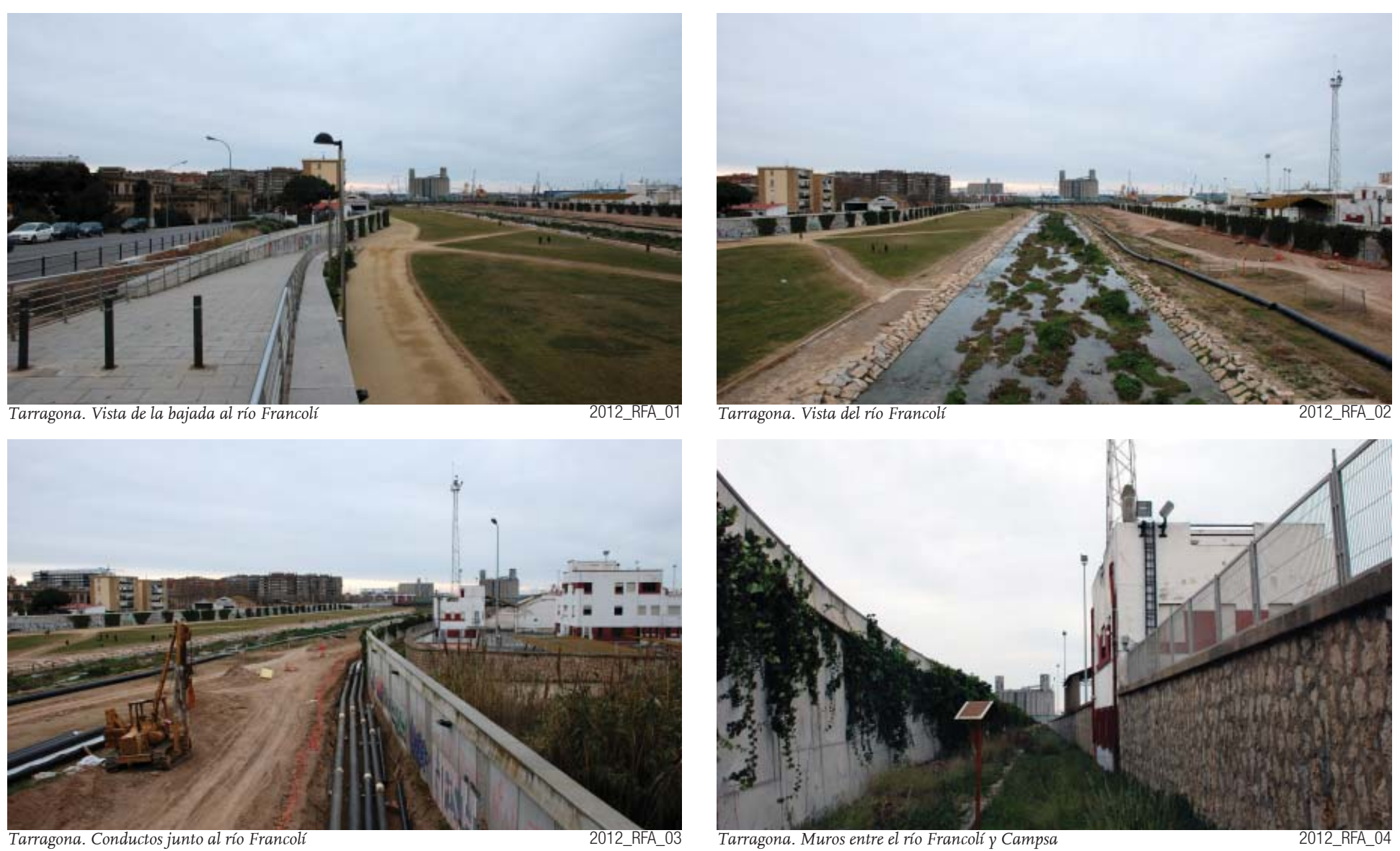


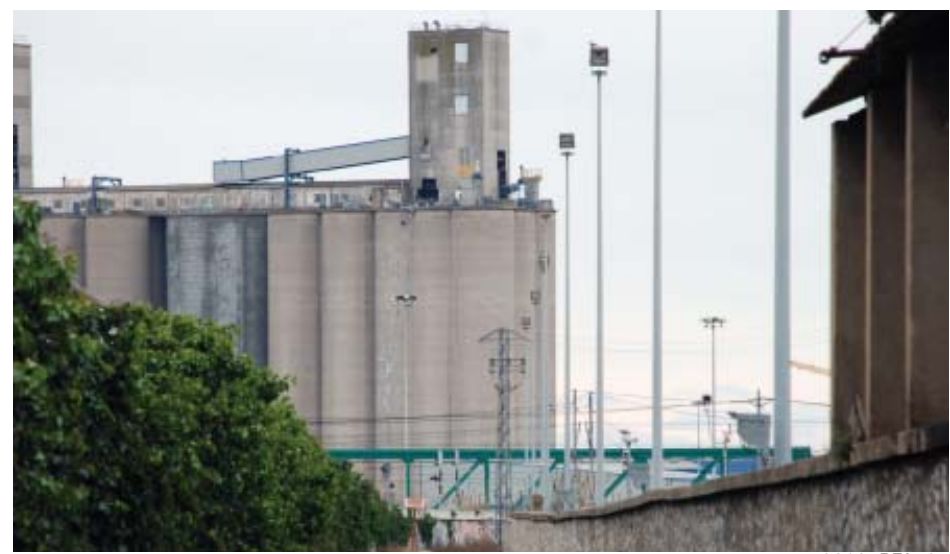

Tarragona. Muros entre el río Francolí y la Industria

2012_RFA_05
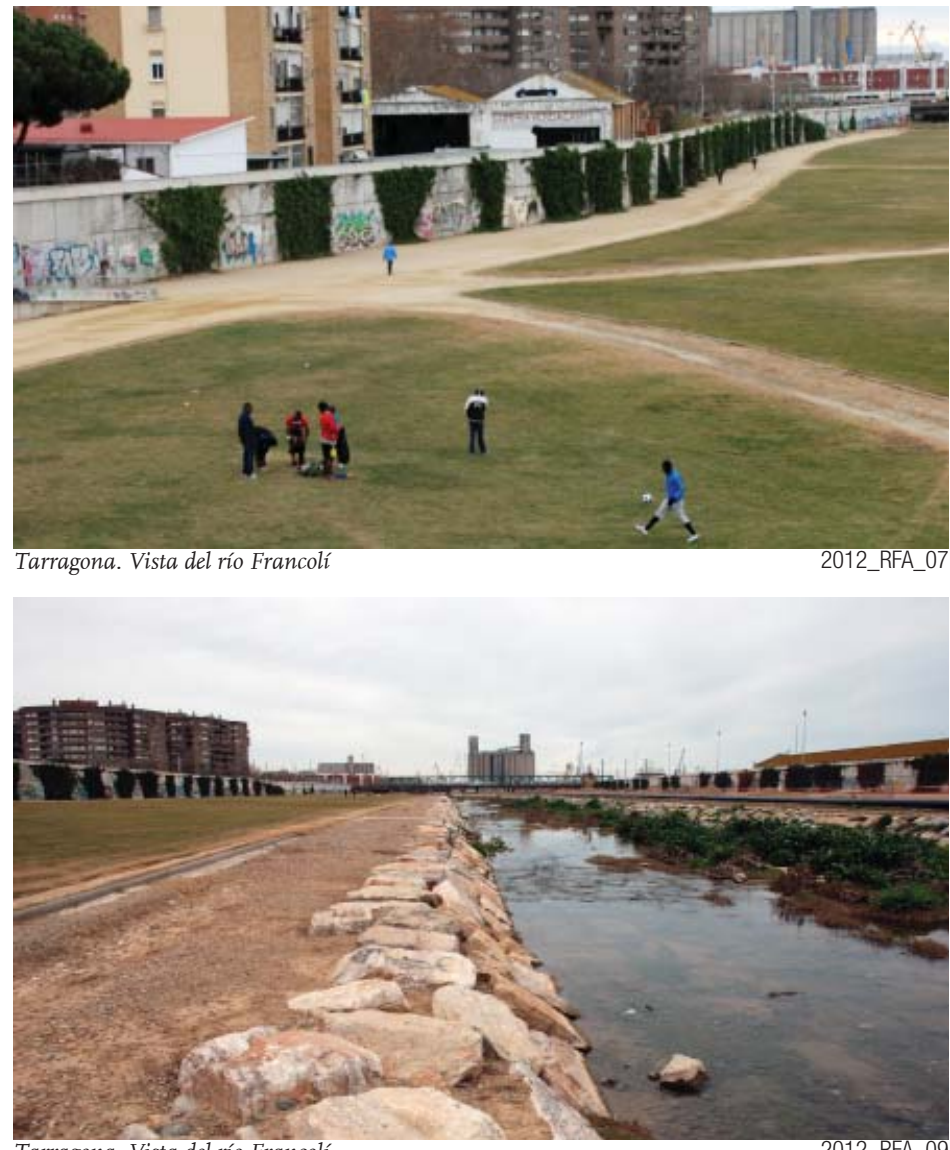

Tarragona. Vista del río Francolí

2012_RFA_09
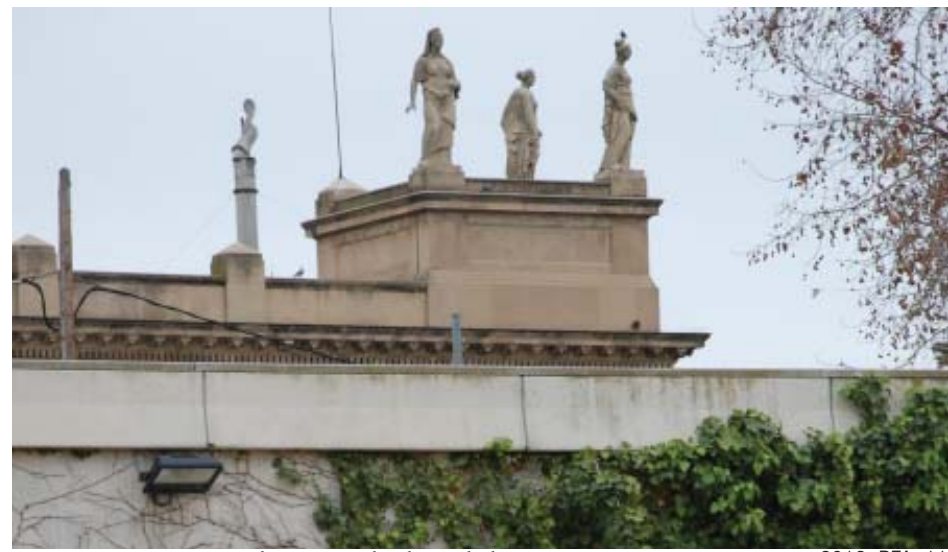

Tarragona. Muros entre el río Francolí Y la ciudad

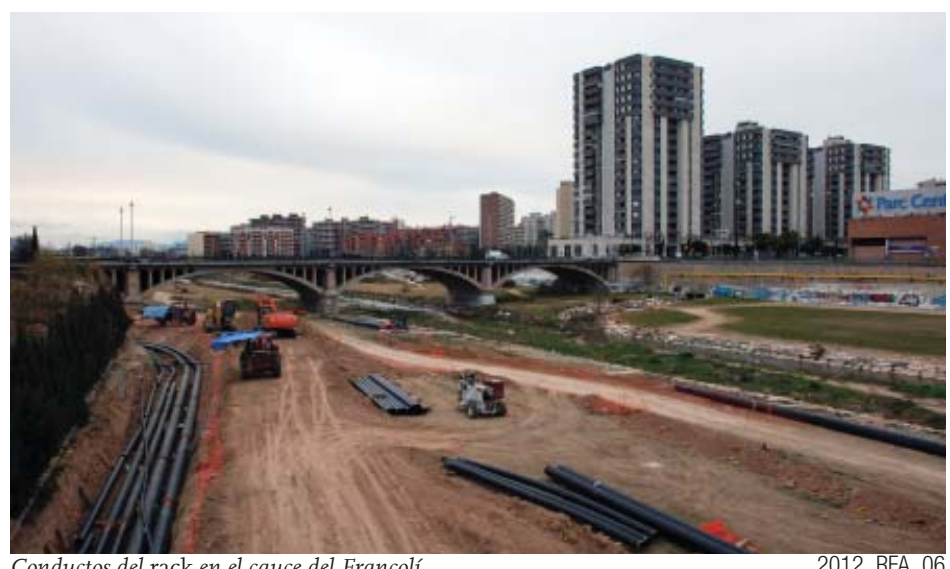

Conductos del rack en el cauce del Francolí

2012_RFA_06
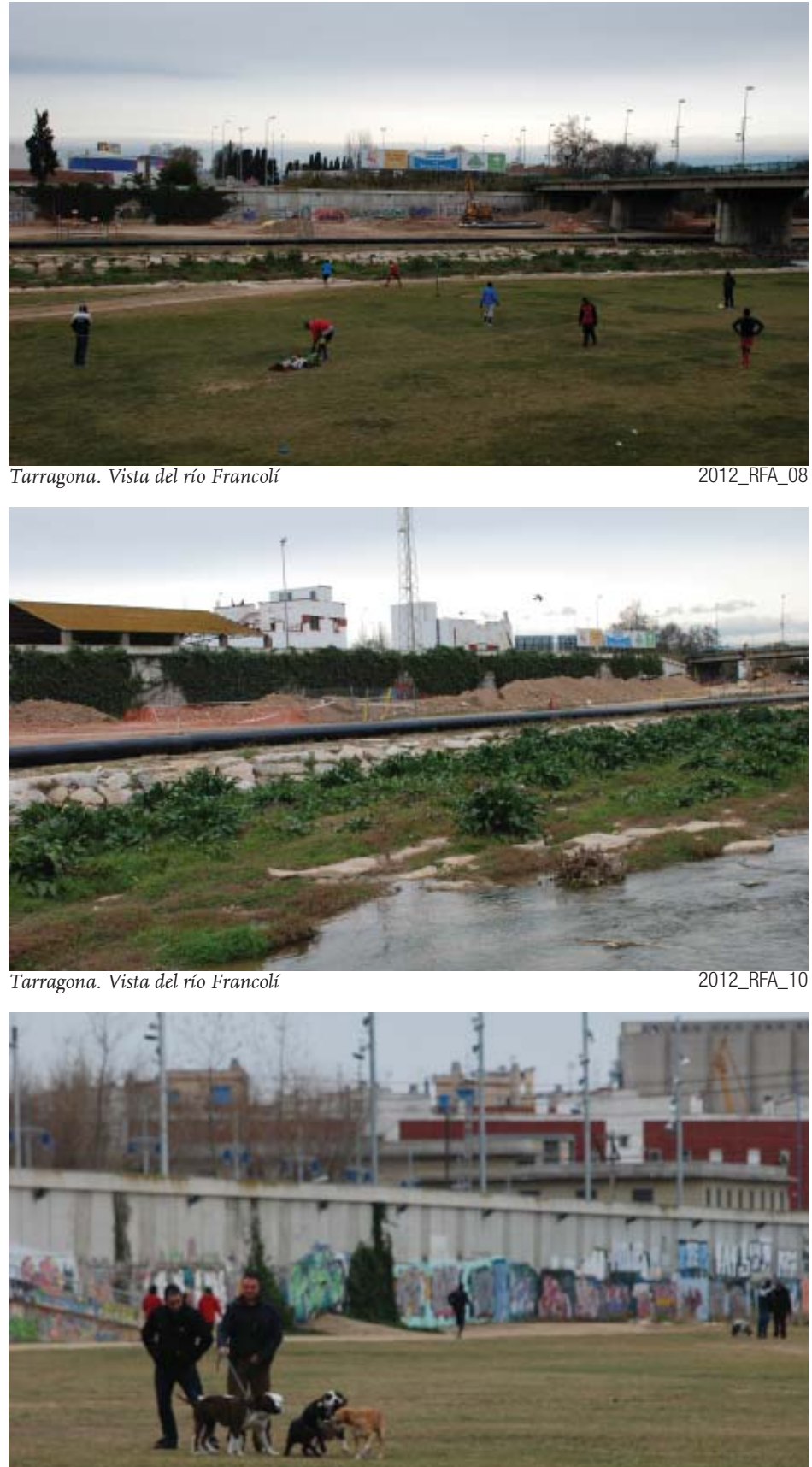

Tarragona. Vista del río Francolí

2012_RFA_12 

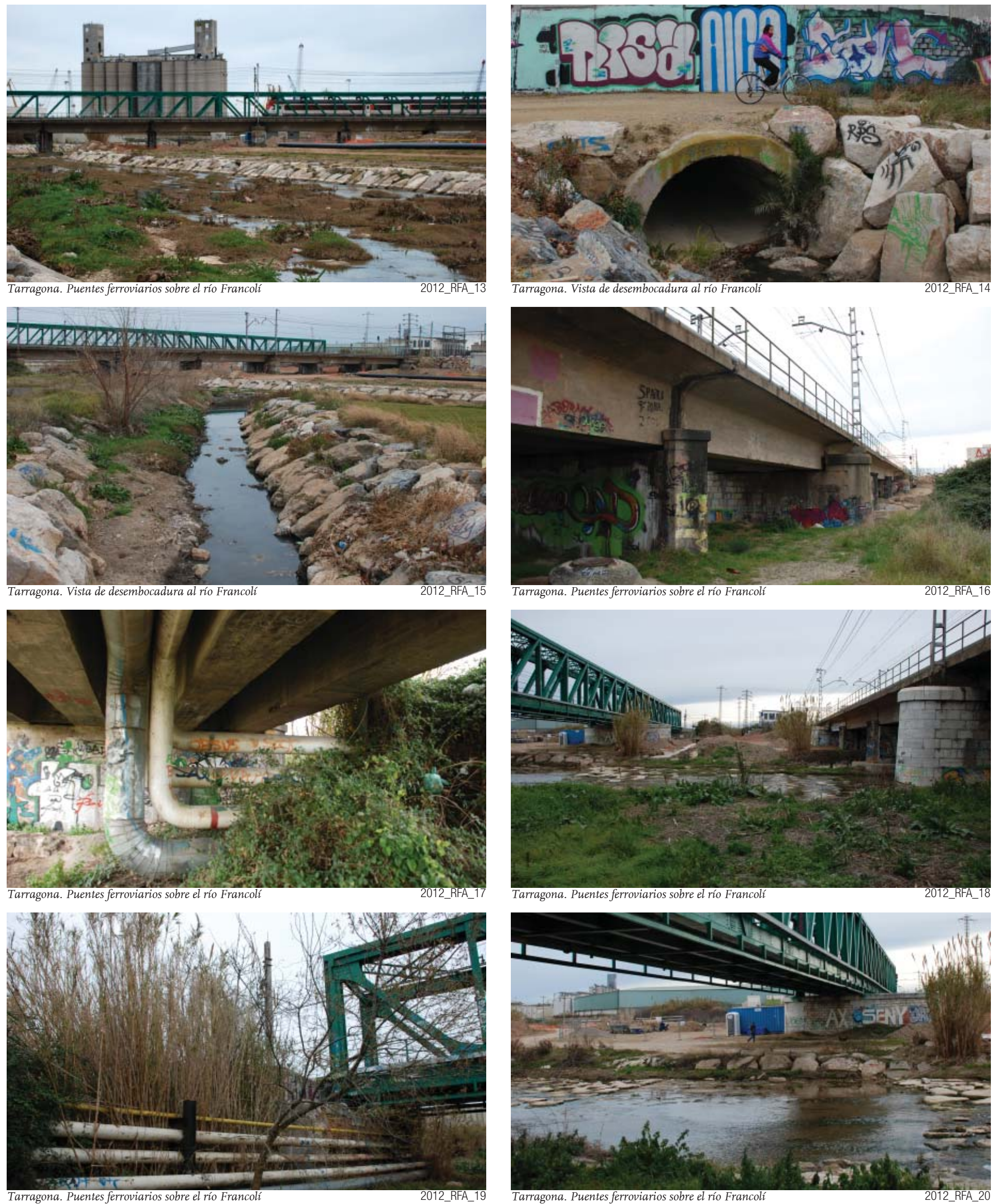


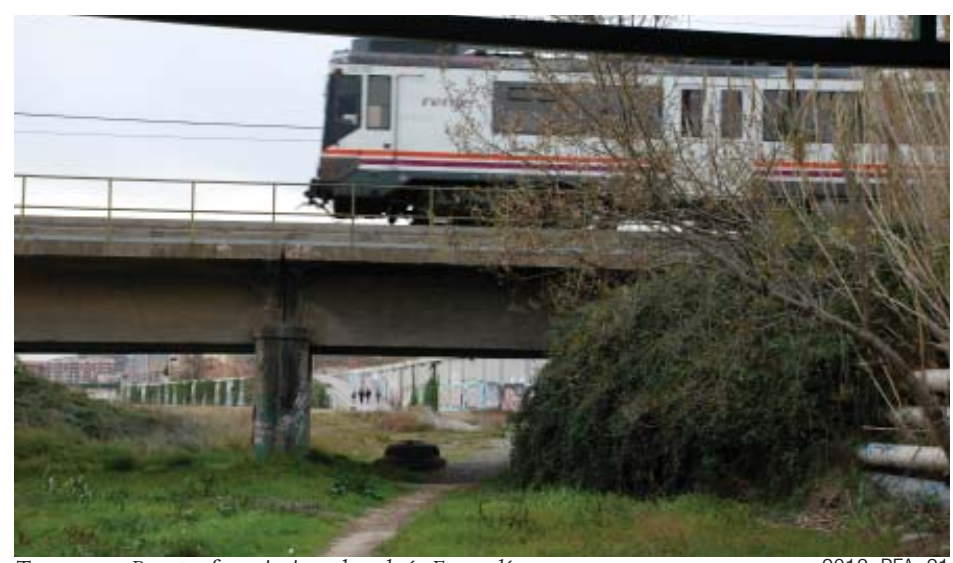

Tarragona. Puentes ferroviarios sobre el río Francolí

2012_RFA_21
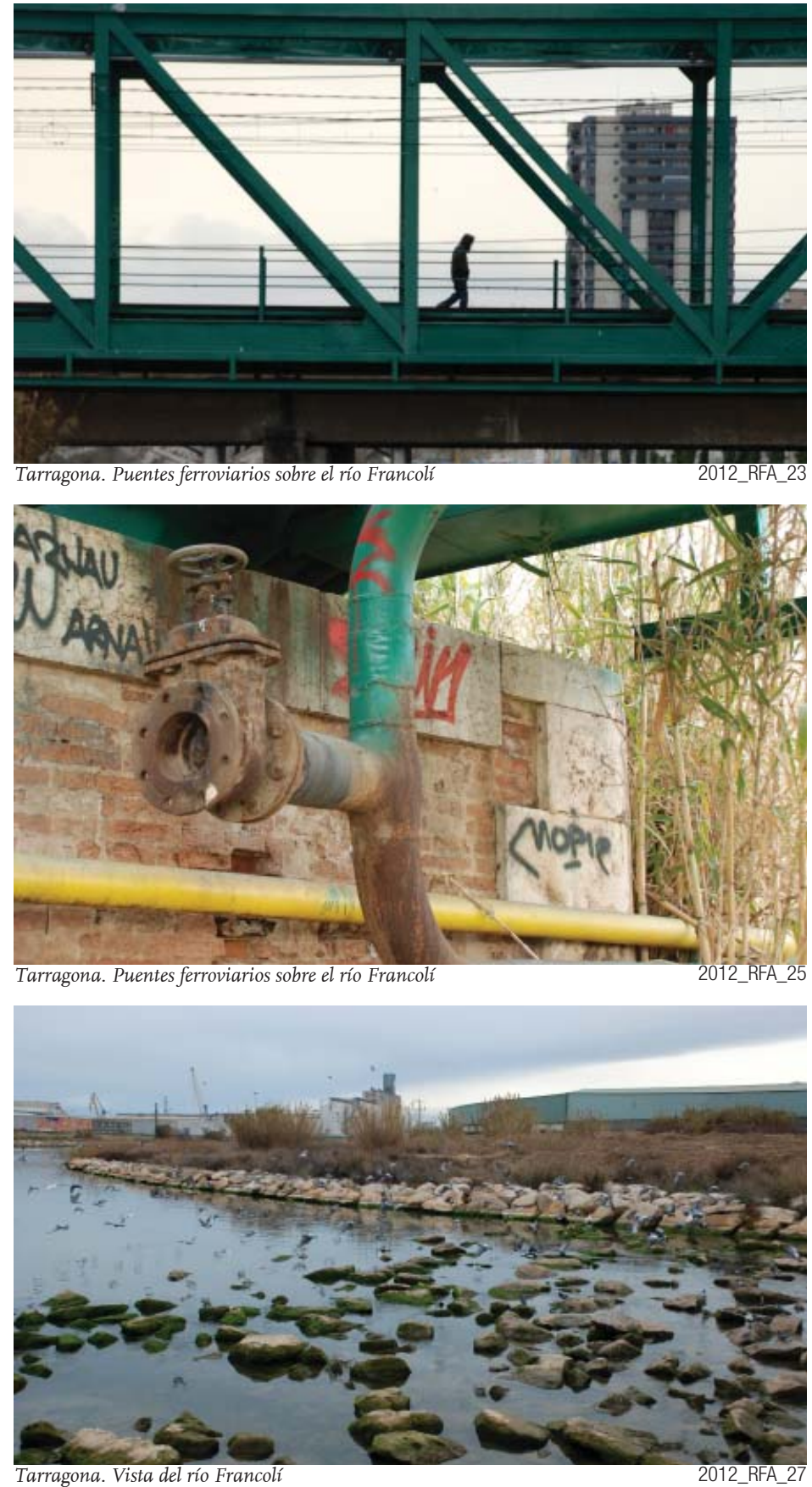
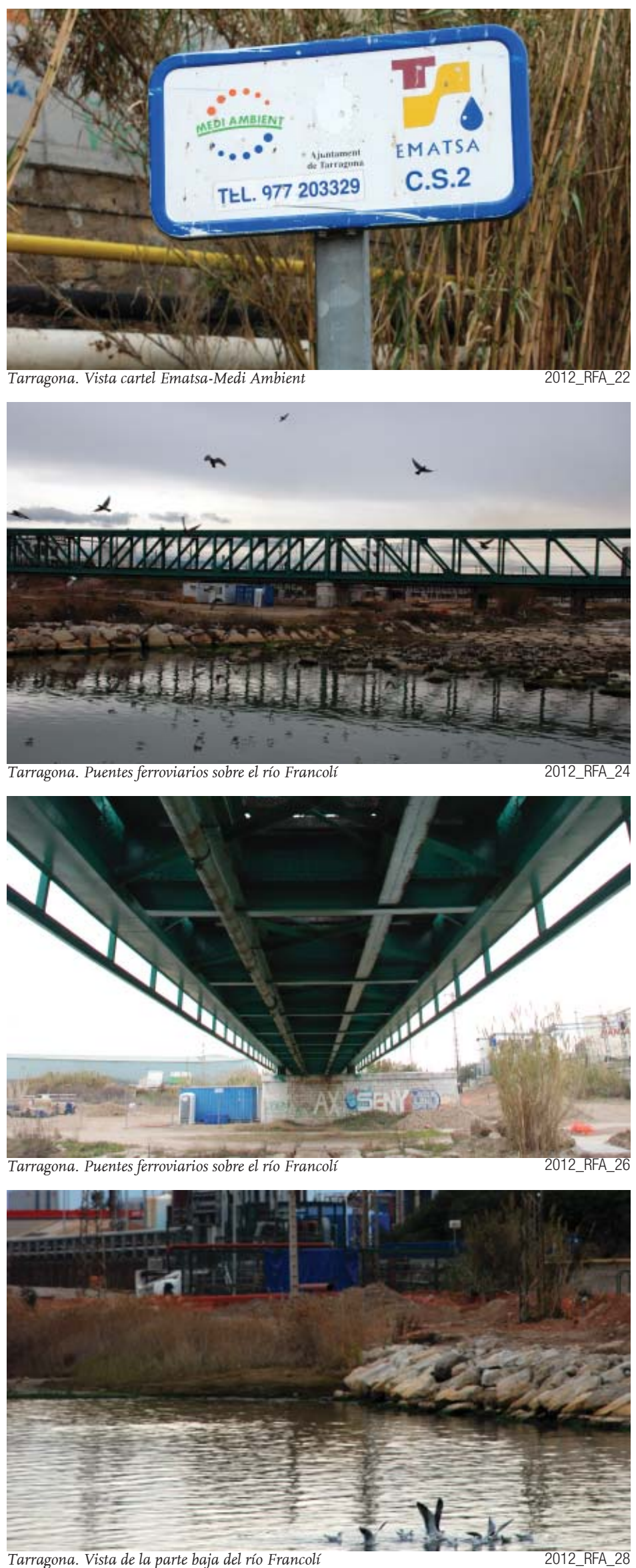

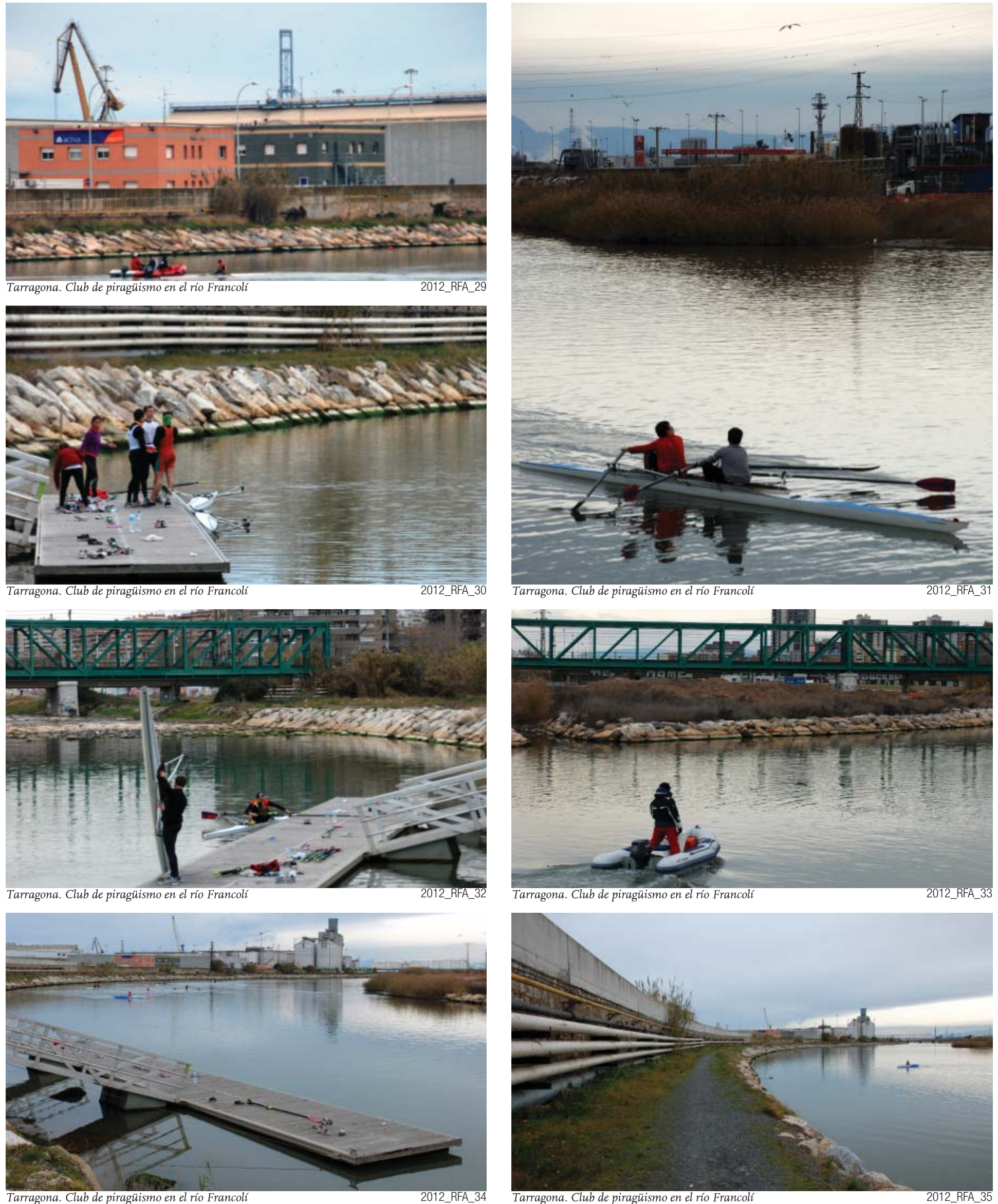


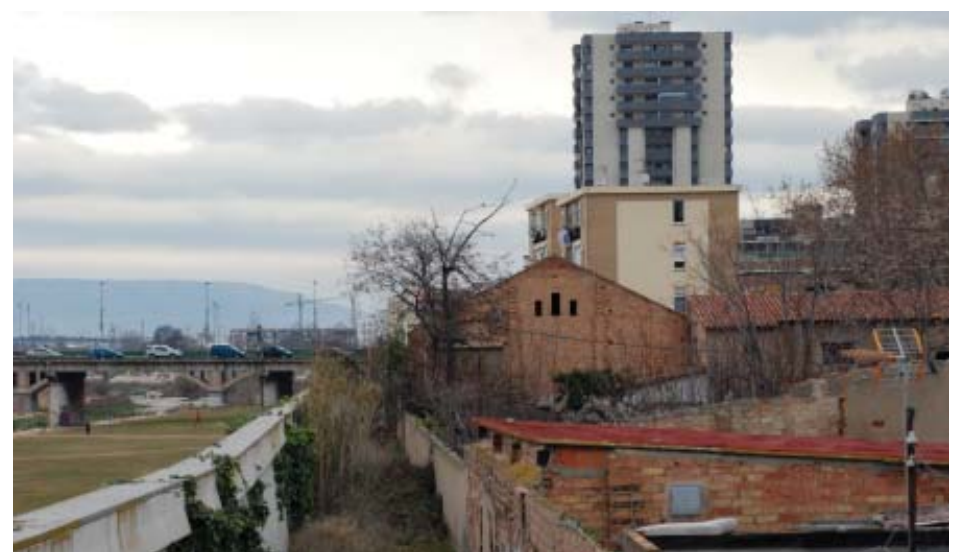

Tarragona. Muros entre el río Francolí y la ciudad

2012_RFA_36

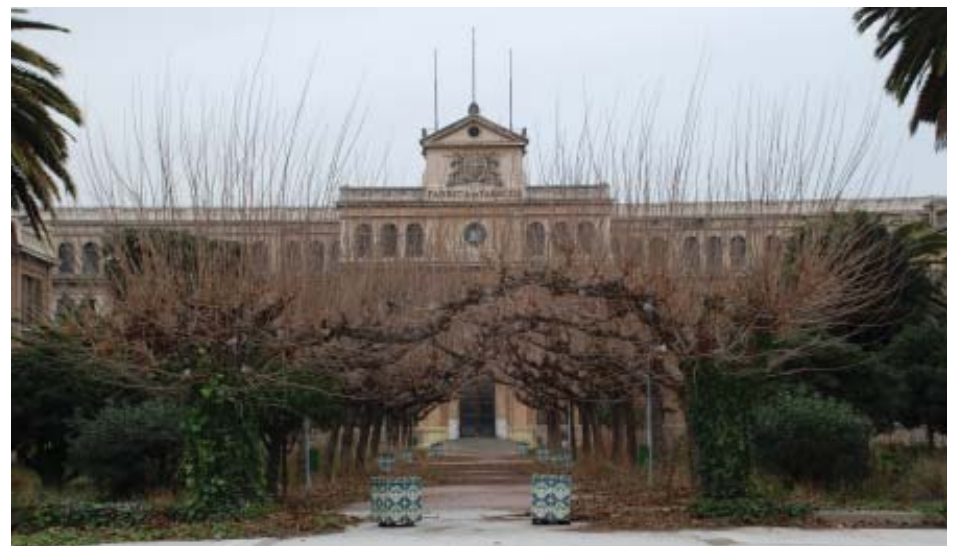

Tabacalera. Vista de la fachada

2012_RFA_38
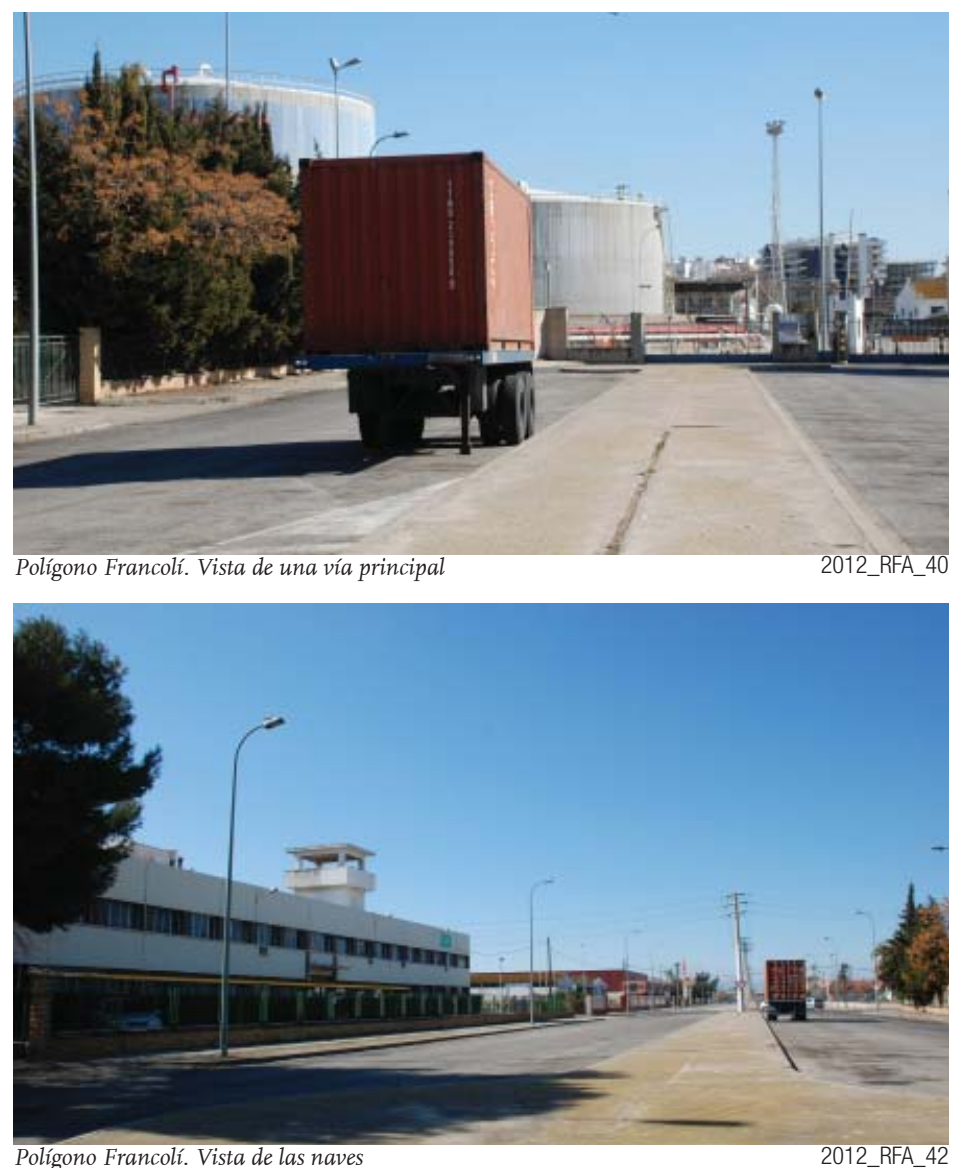

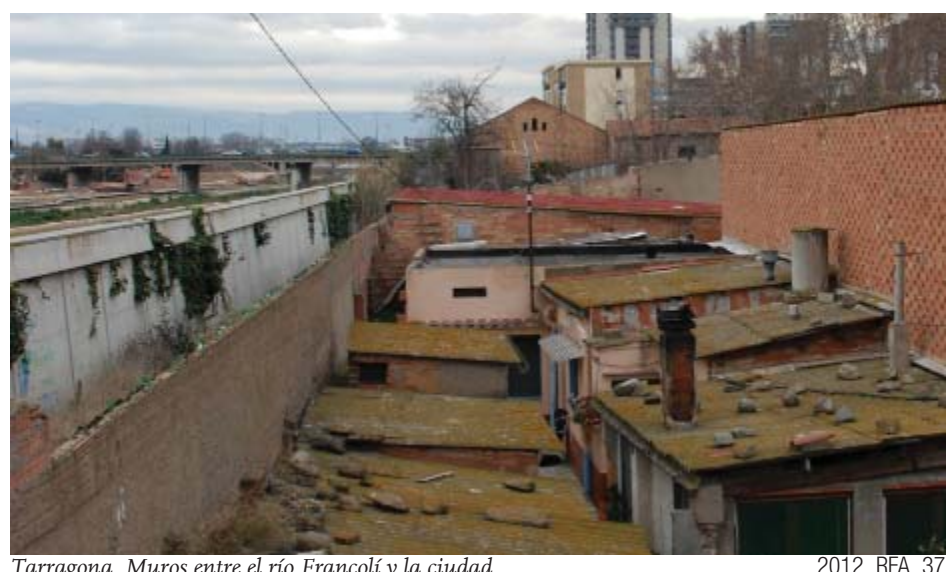

Tarragona. Muros entre el río Francolí y la ciudad

2012_RFA_37
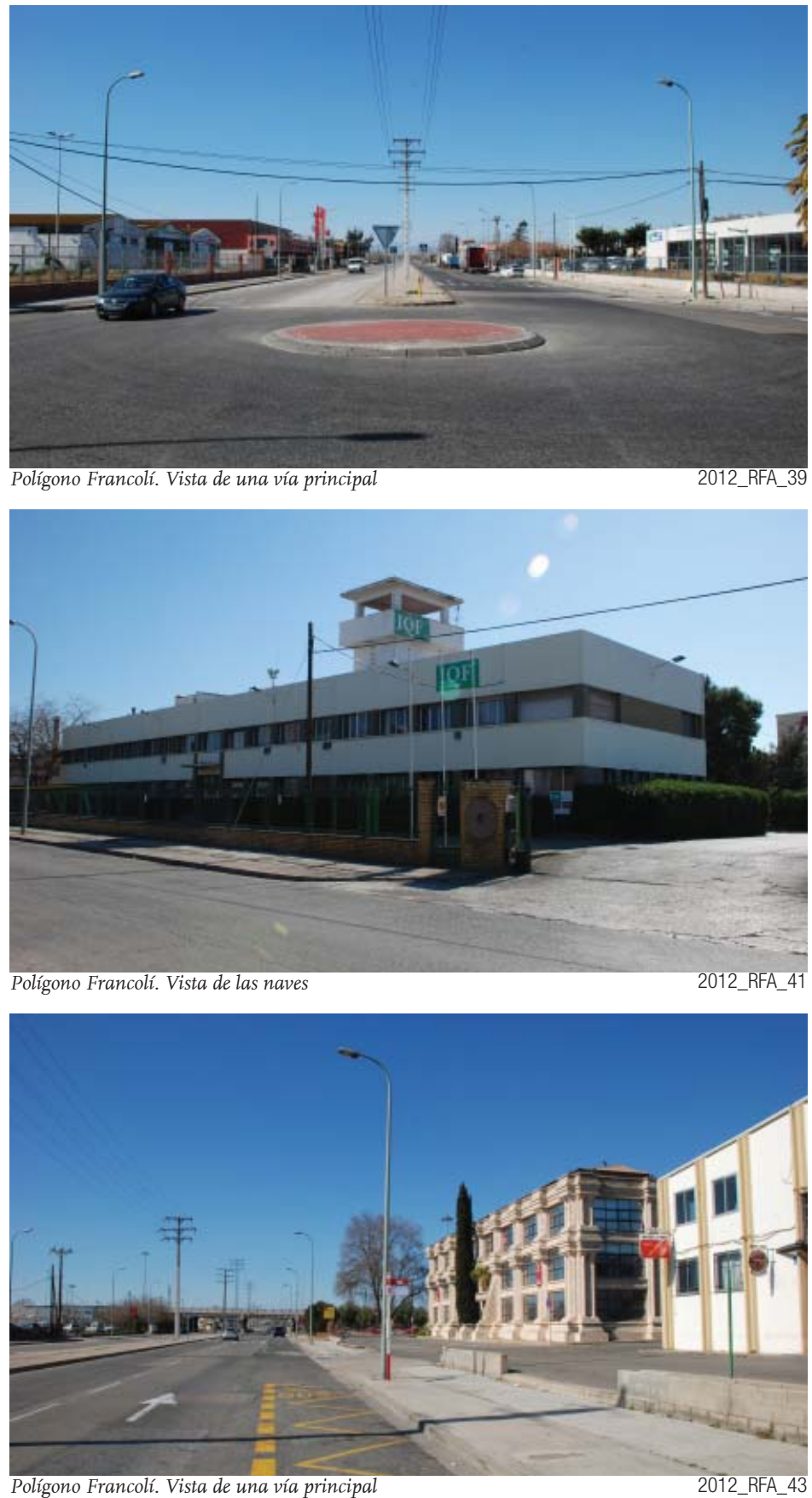


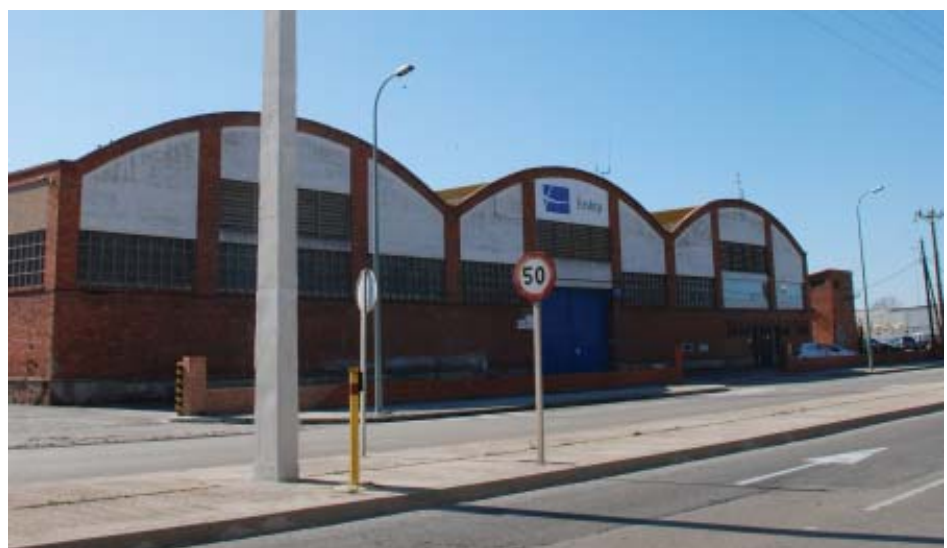

Polígono Francolí. Vista de las naves

2012_RFA_44

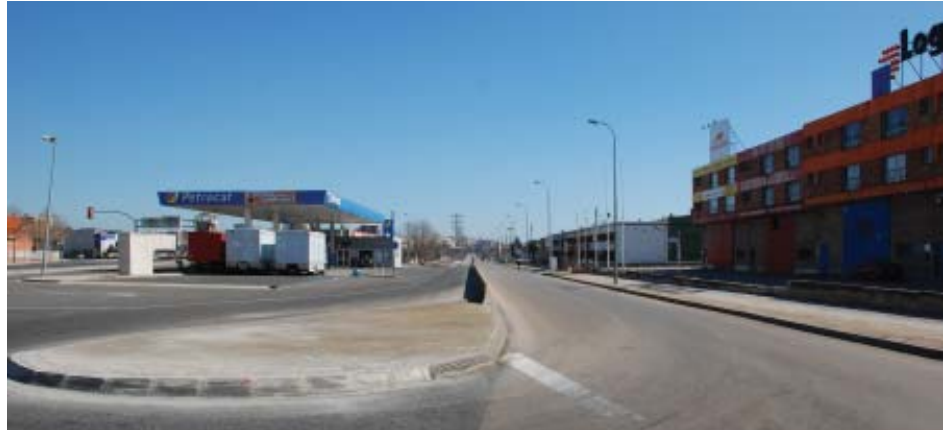

Polígono Francolí. Vista de una vía principal

2012 RFA 46
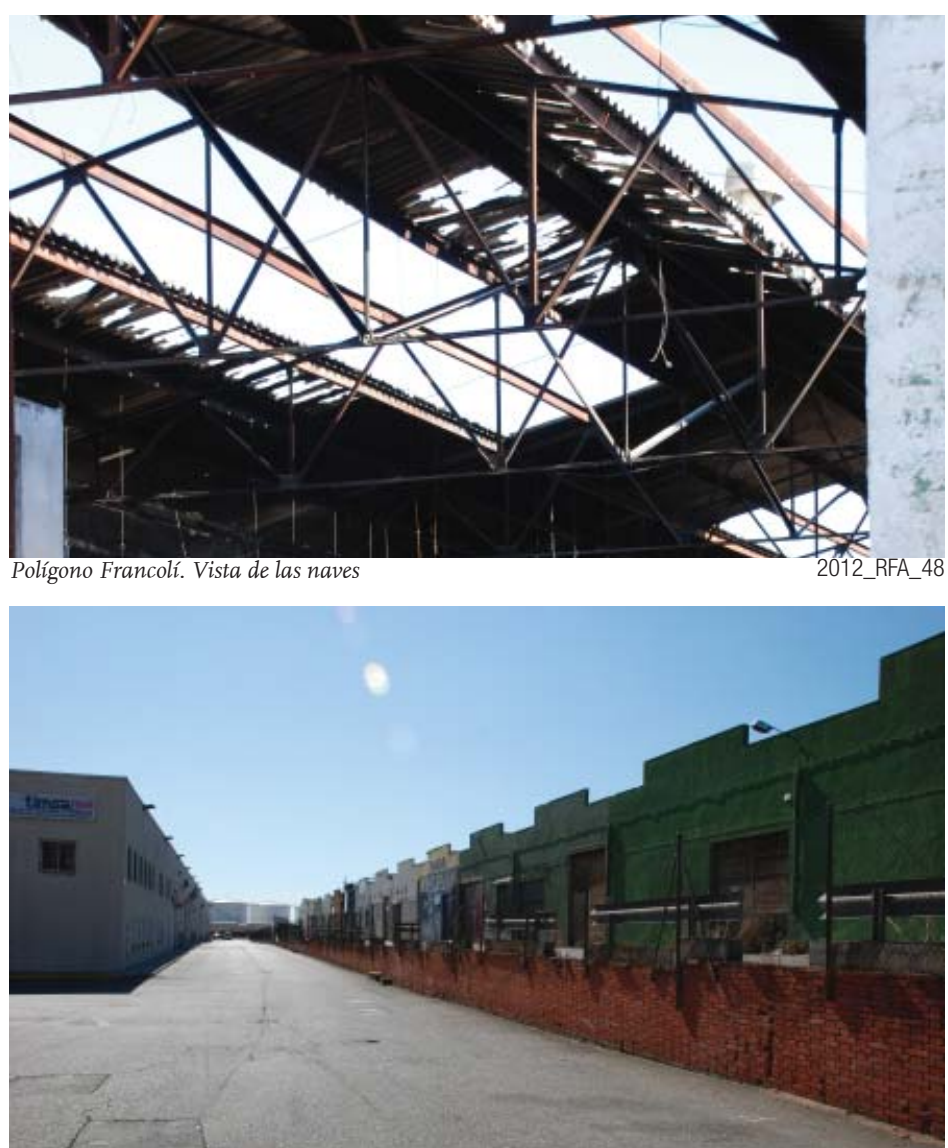

Polígono Francolí. Vista de las naves

2012_RFA_50
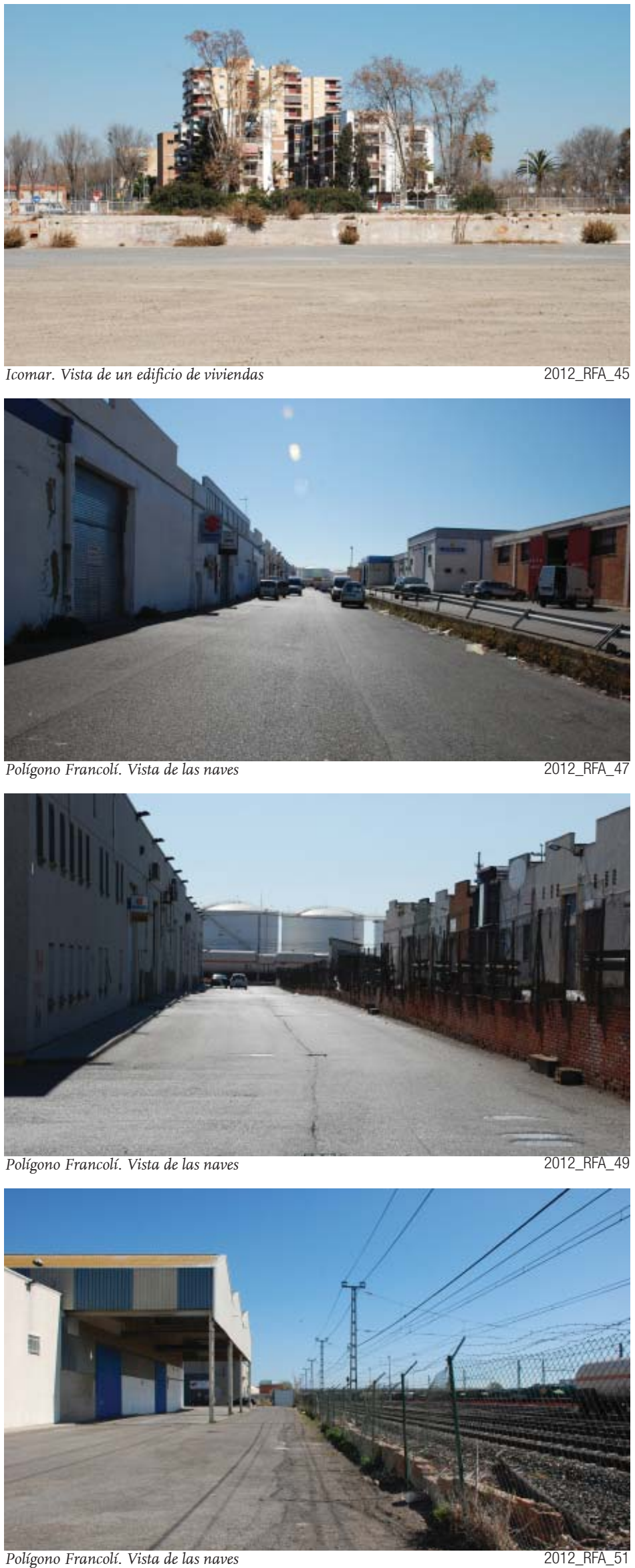

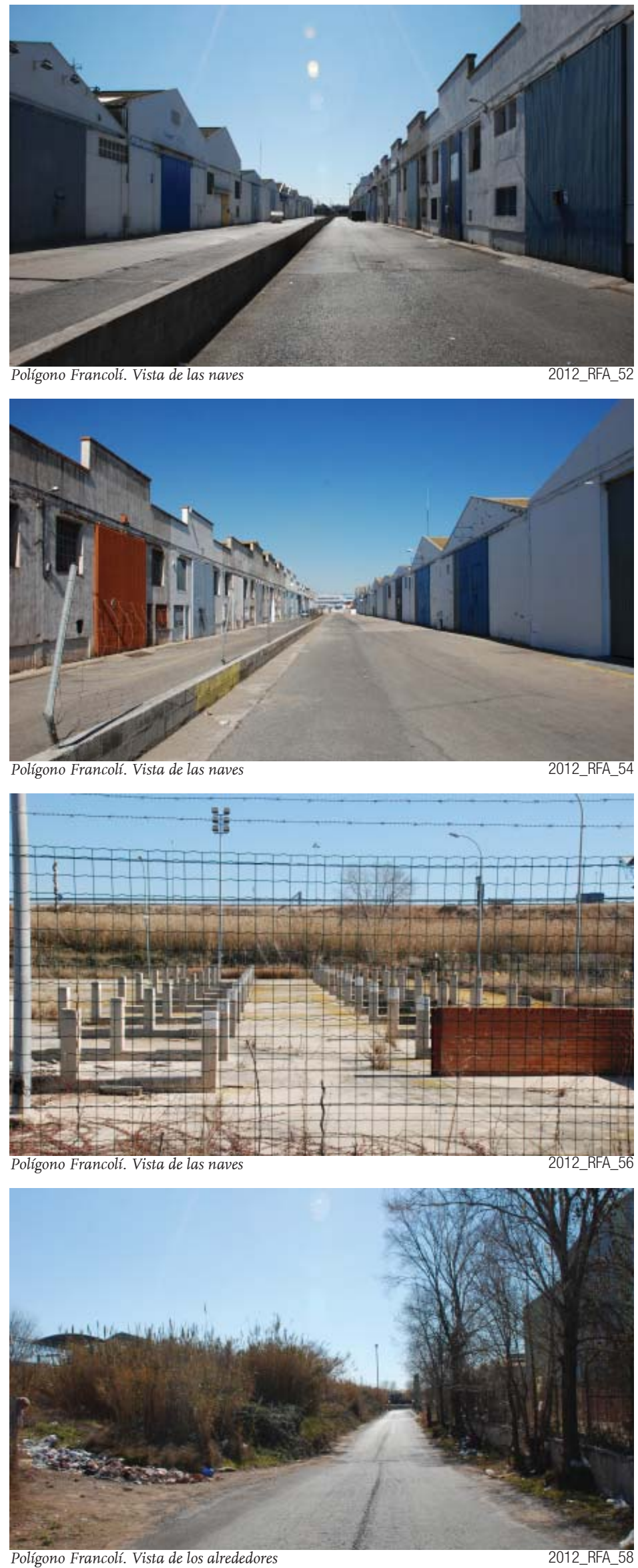

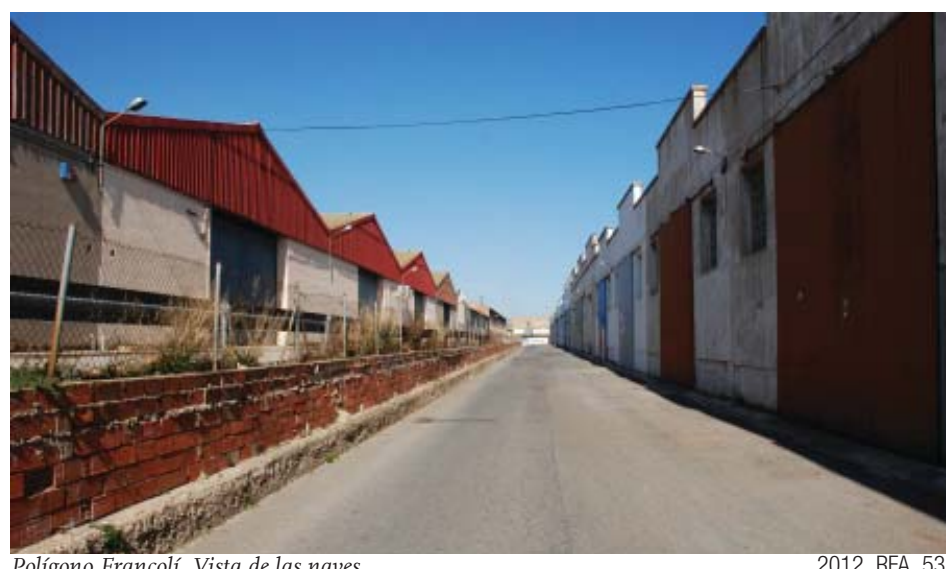

Polígono Francolí. Vista de las naves

2012_RFA 53
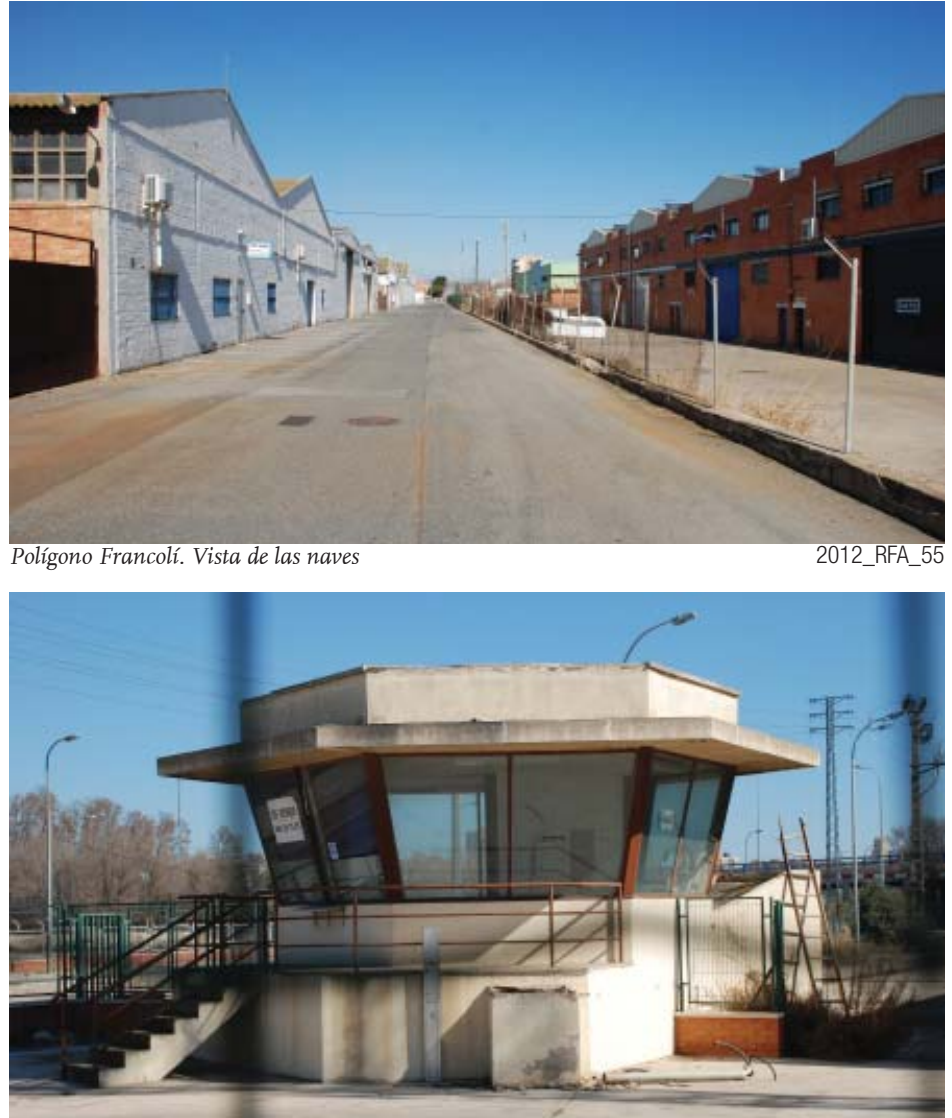

Polígono Francolí. Vista de las naves

2012_RFA_57

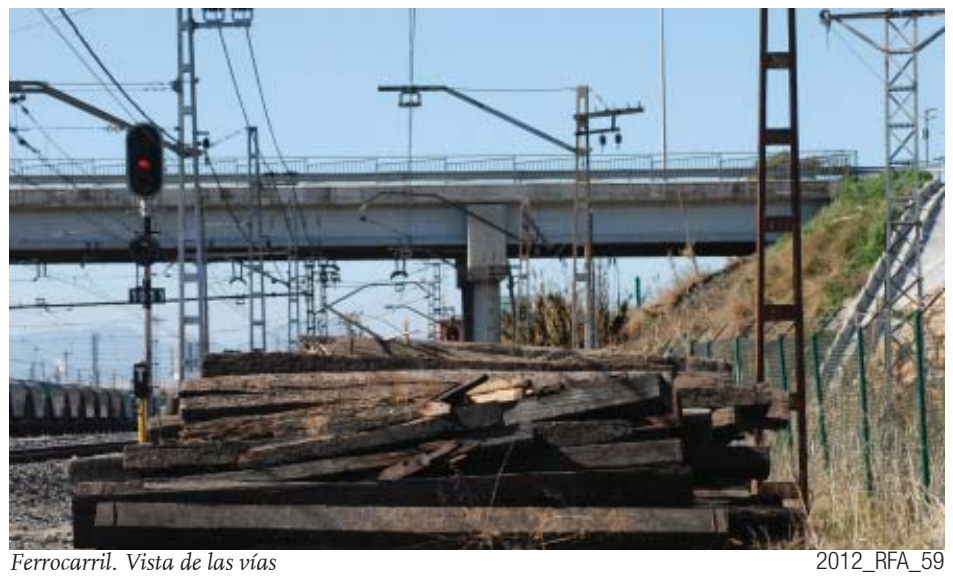



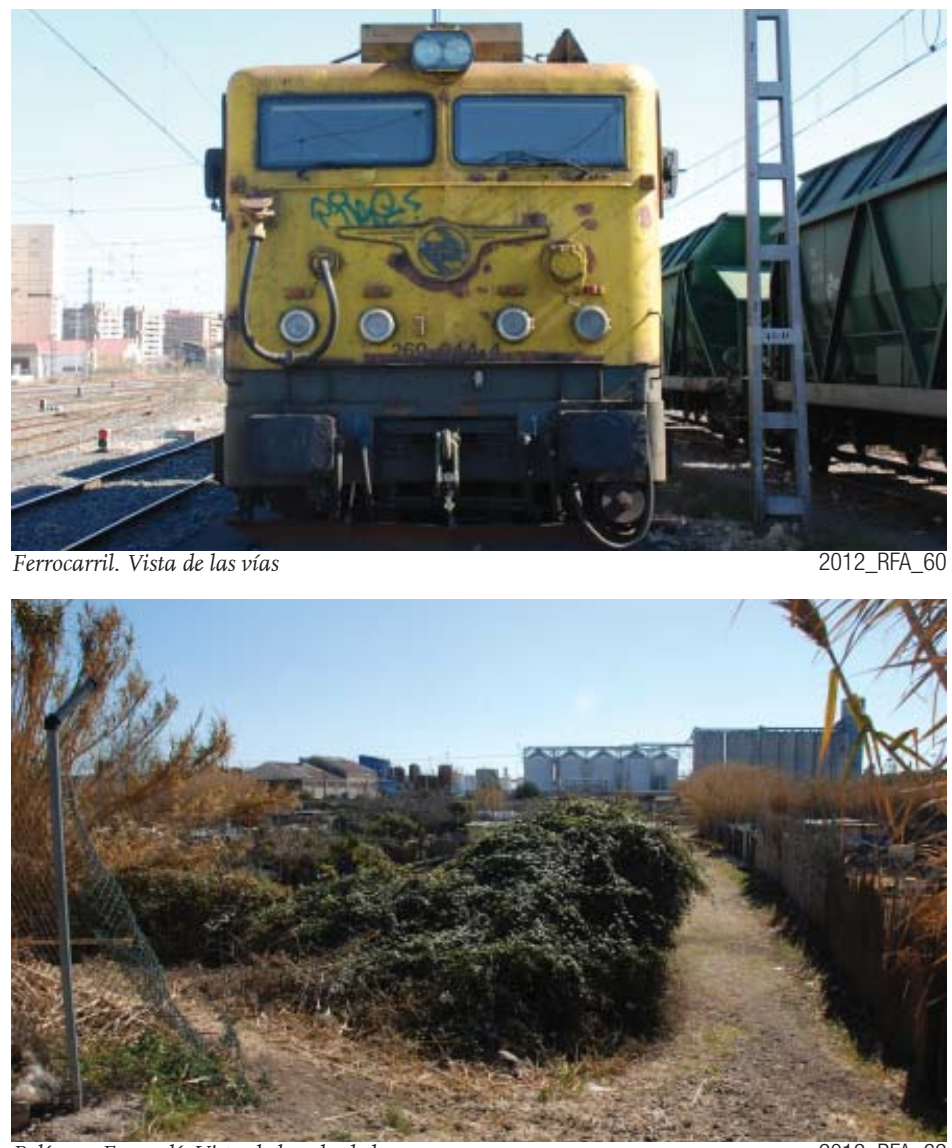

Poligono Francolí. Vista de los alrededores

2012_RFA_62
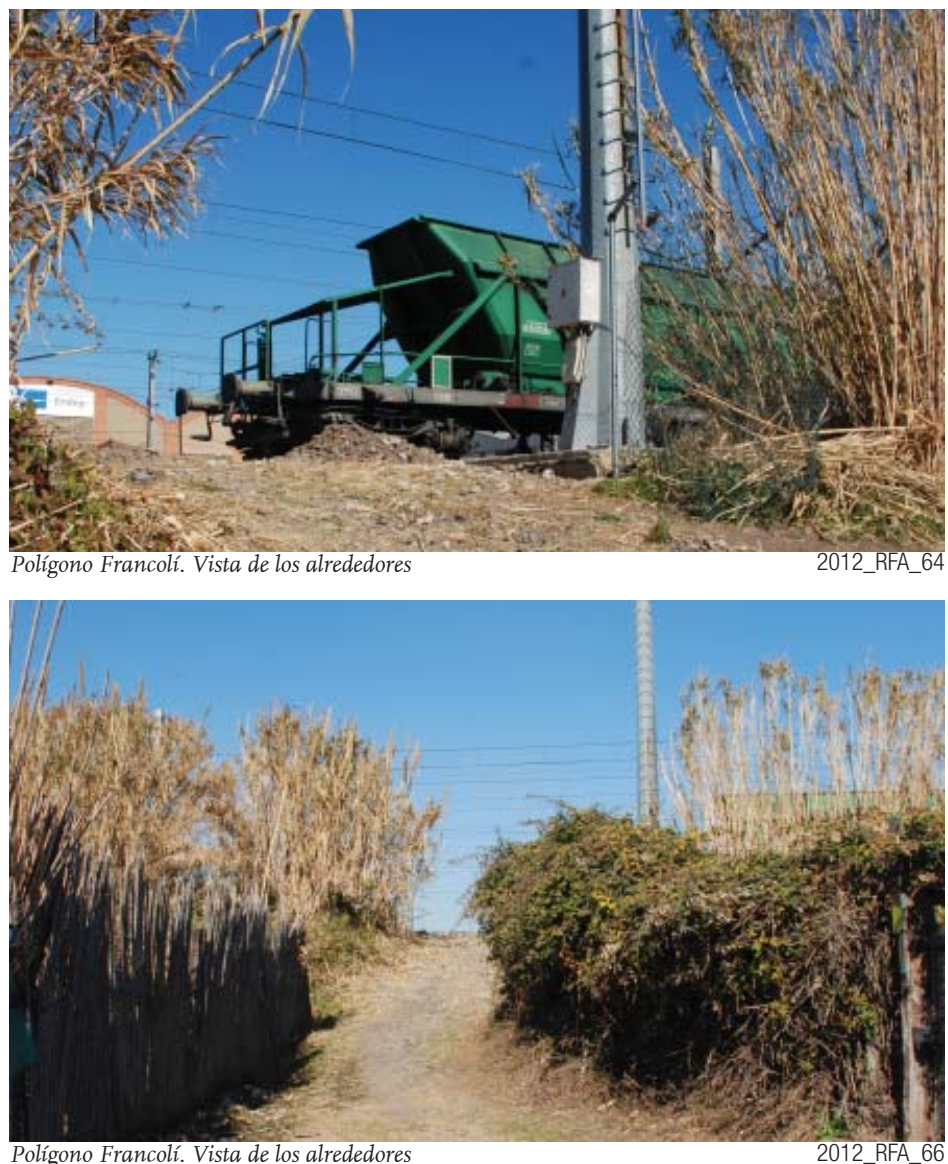
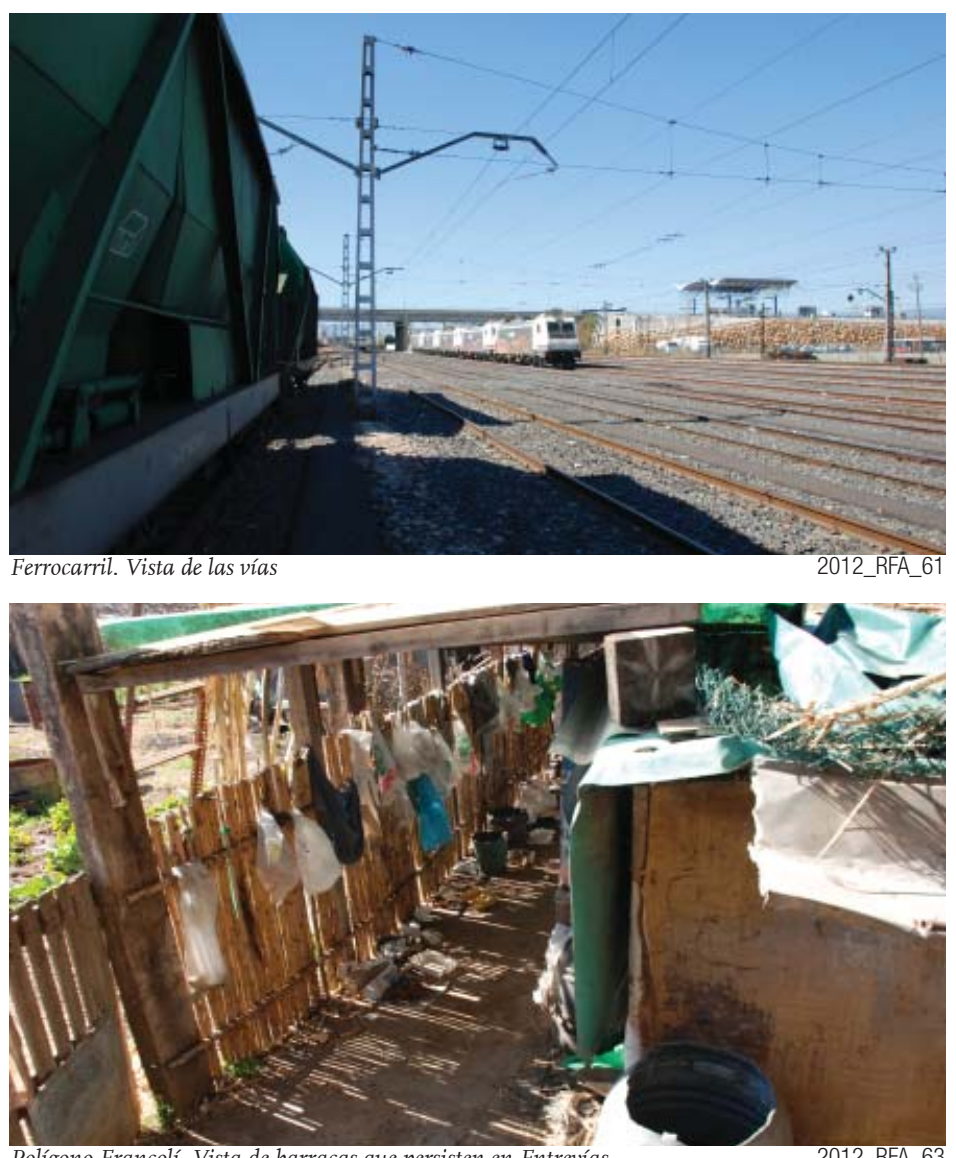

Polígono Francolí. Vista de barracas que persisten en Entrevía

2012_RFA_63

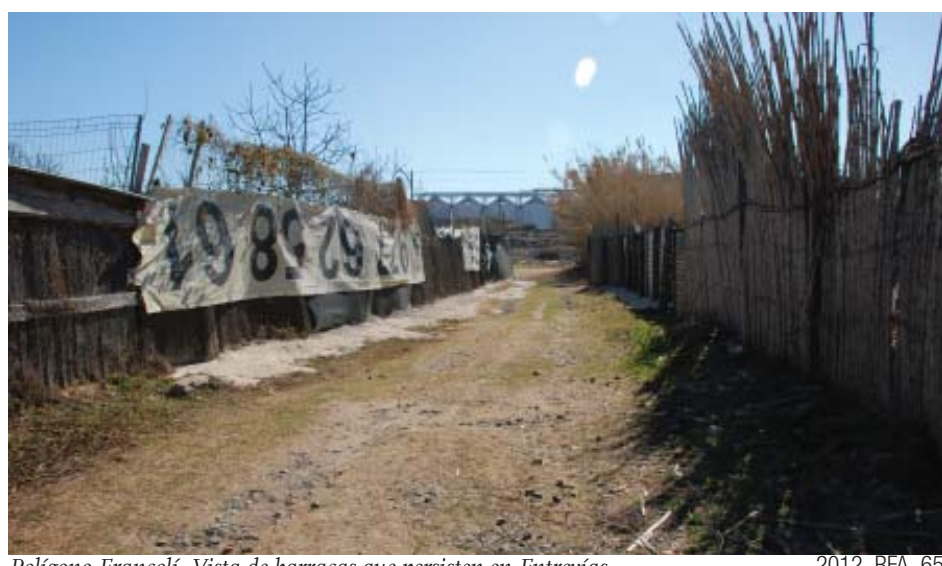

Polígono Francolí Vista de barracas que persisten en Entroúas

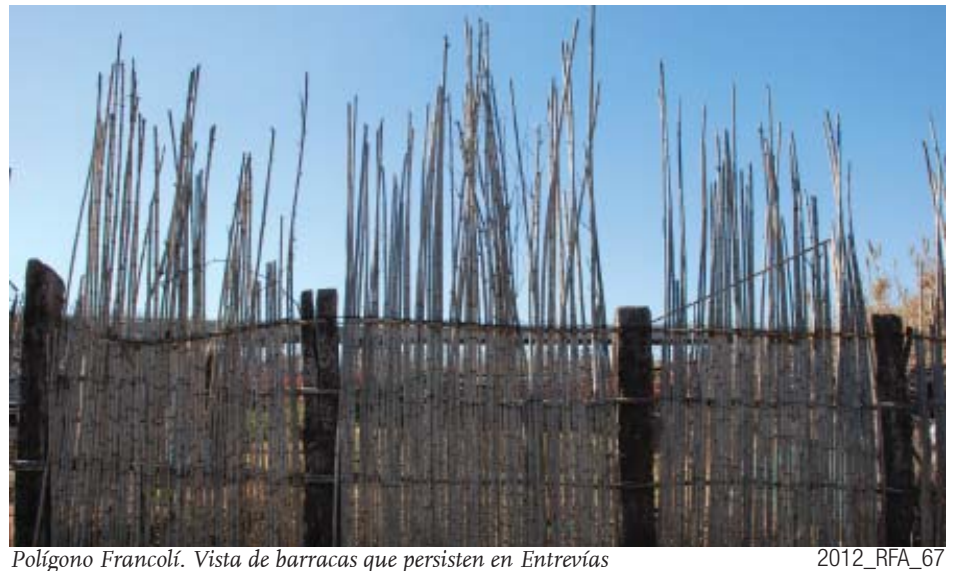



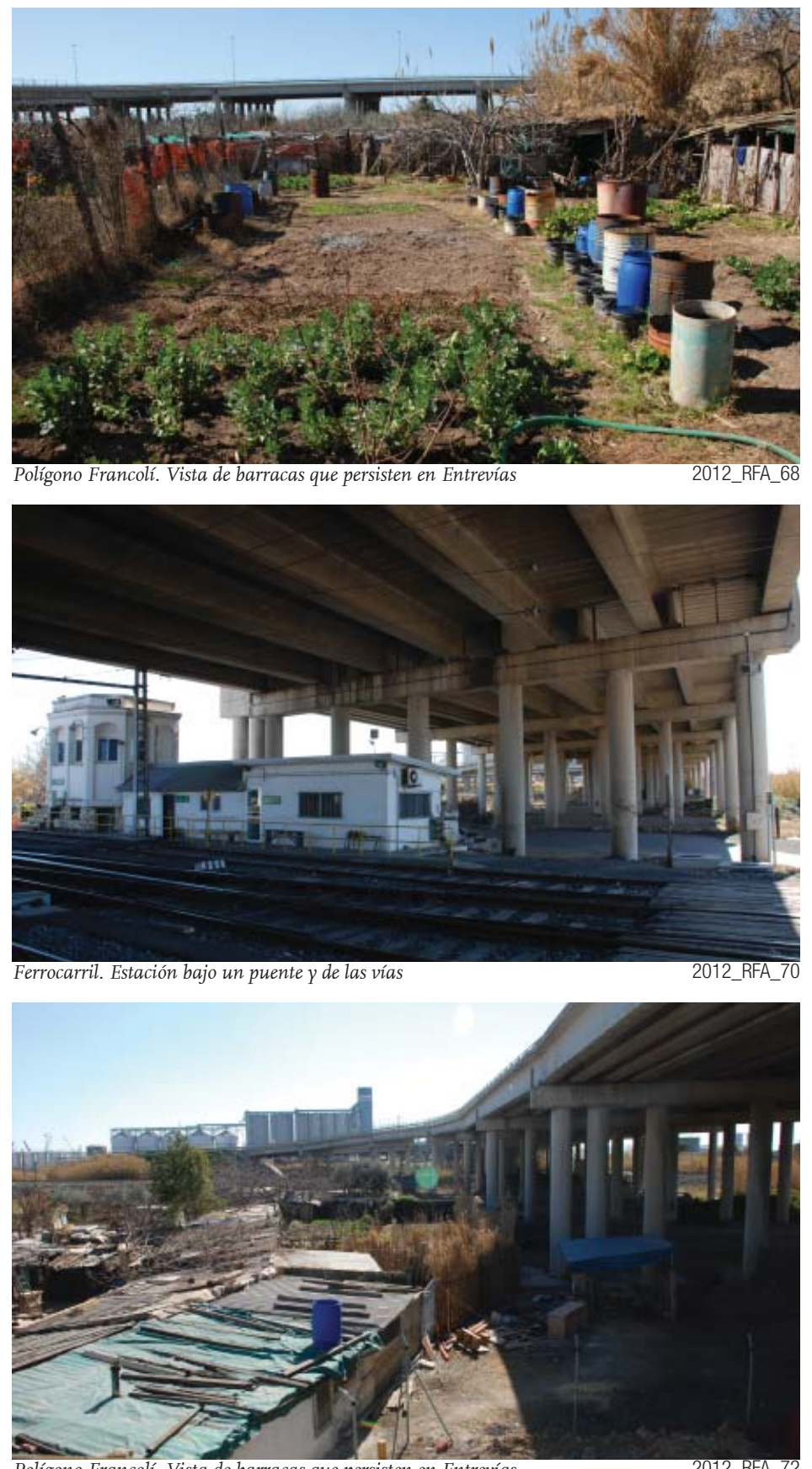

Polígono Francolí. Vista de barracas que persisten en Entrevías

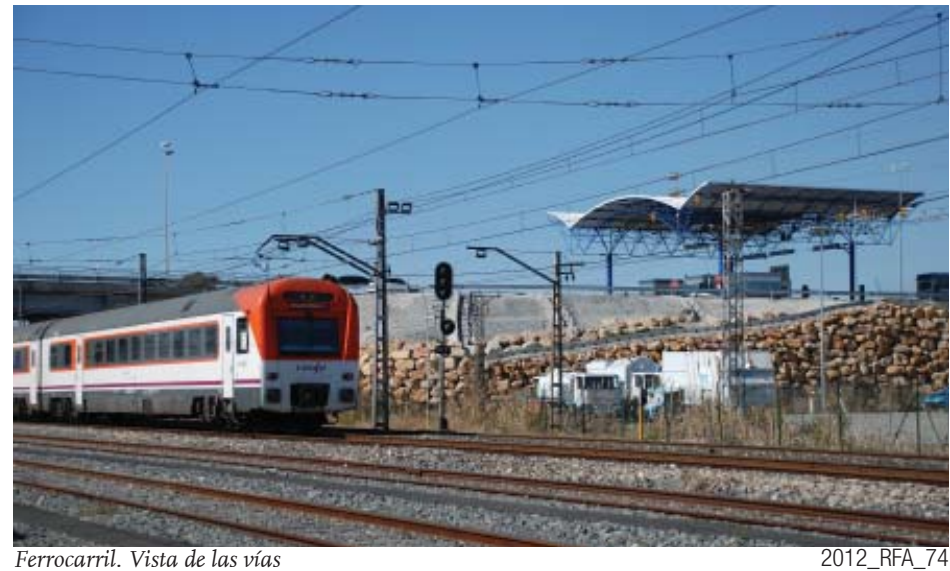

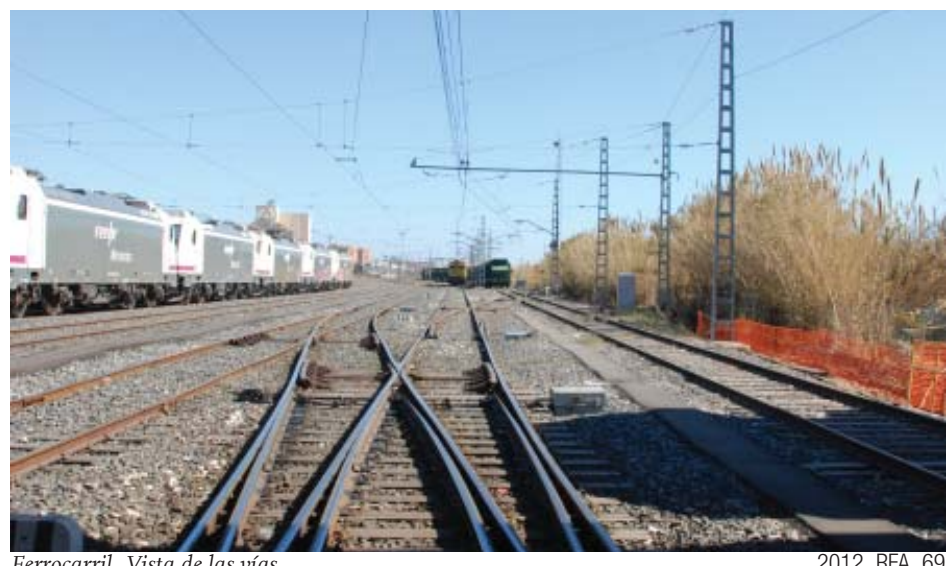

Ferrocarril. Vista de las vías

2012_RFA 69
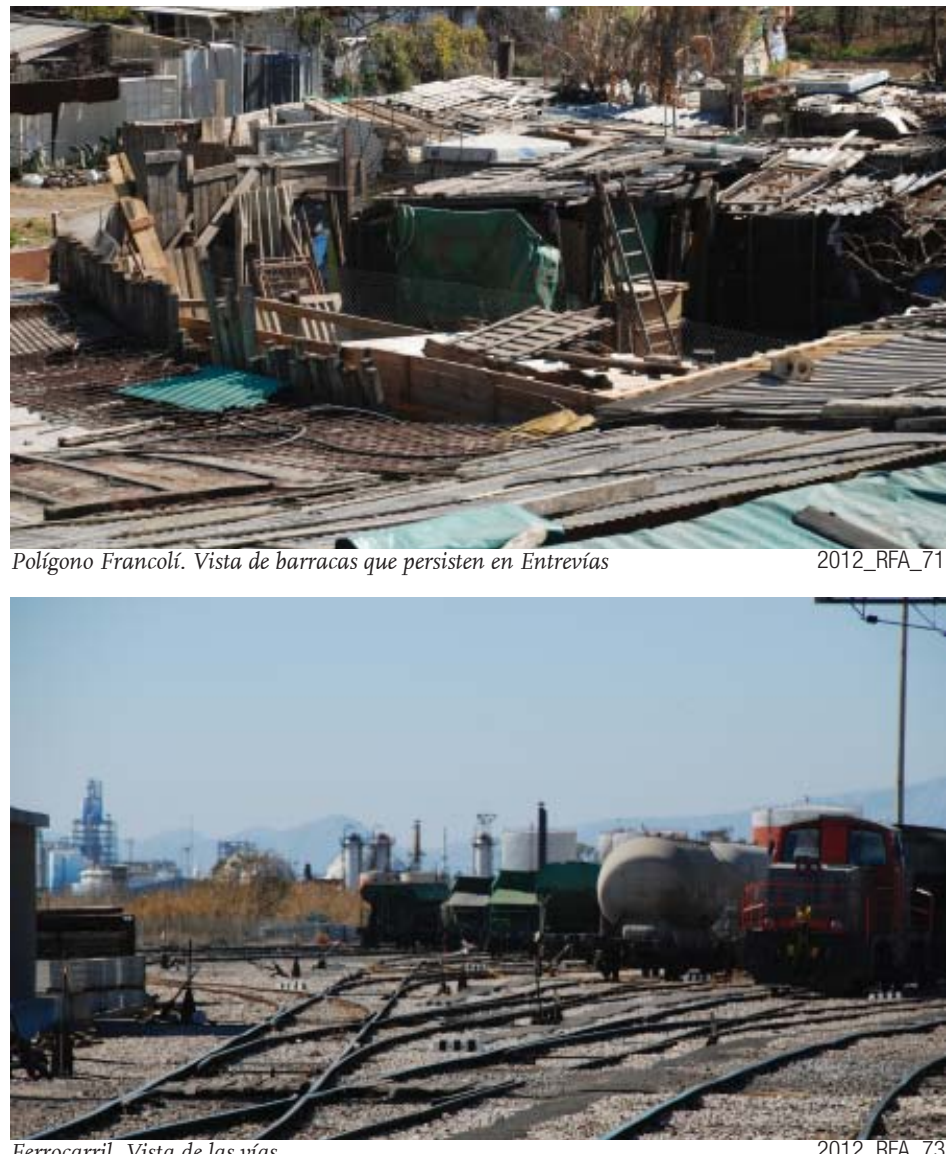

Ferrocarril. Vista de las vías

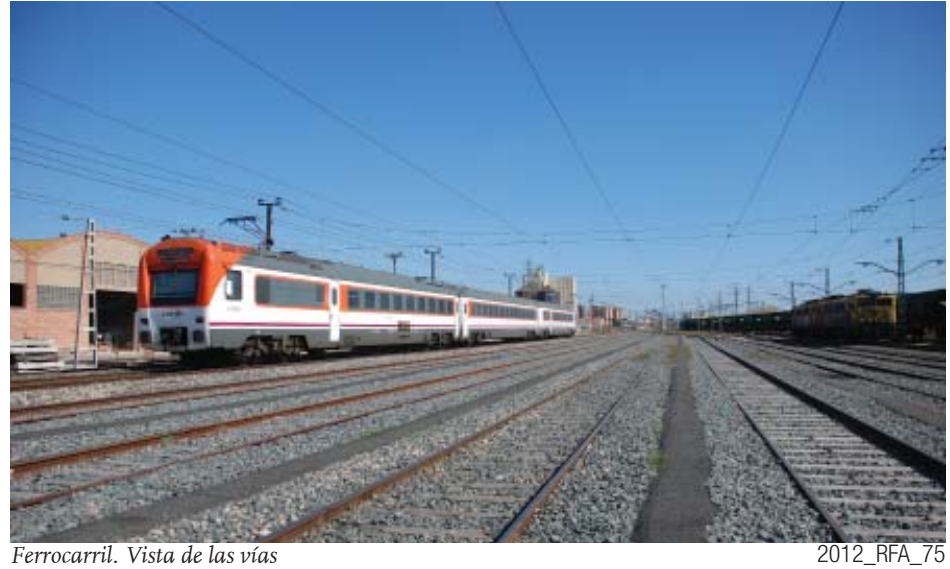



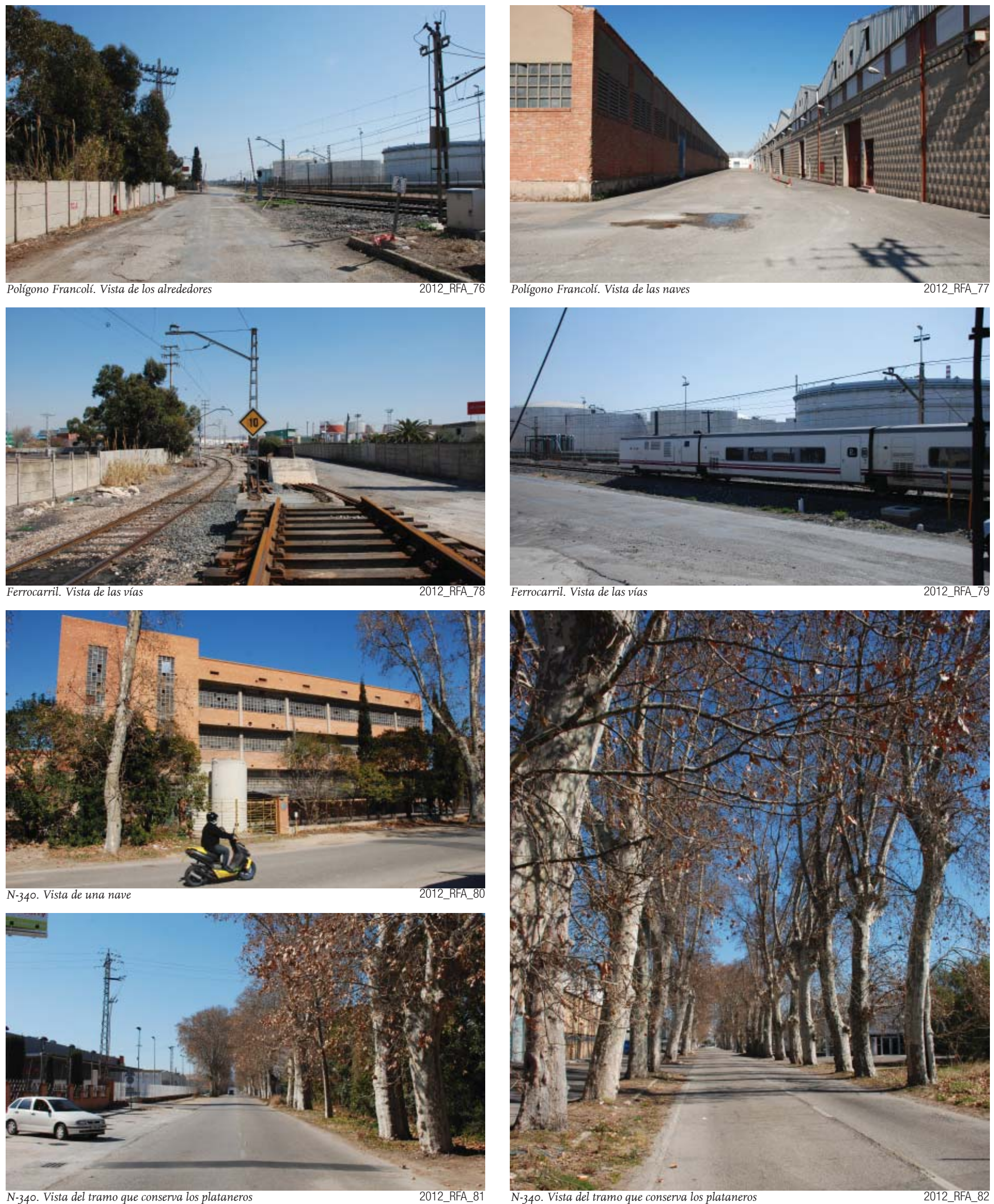

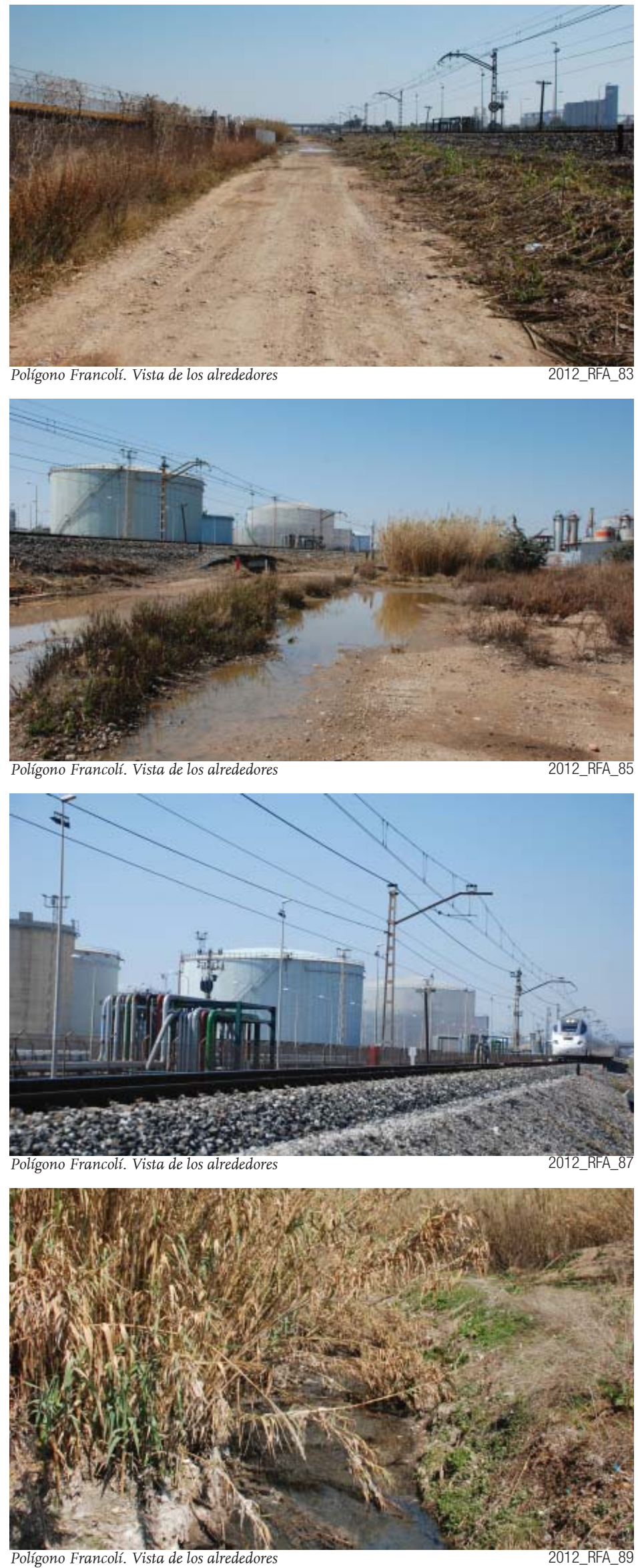
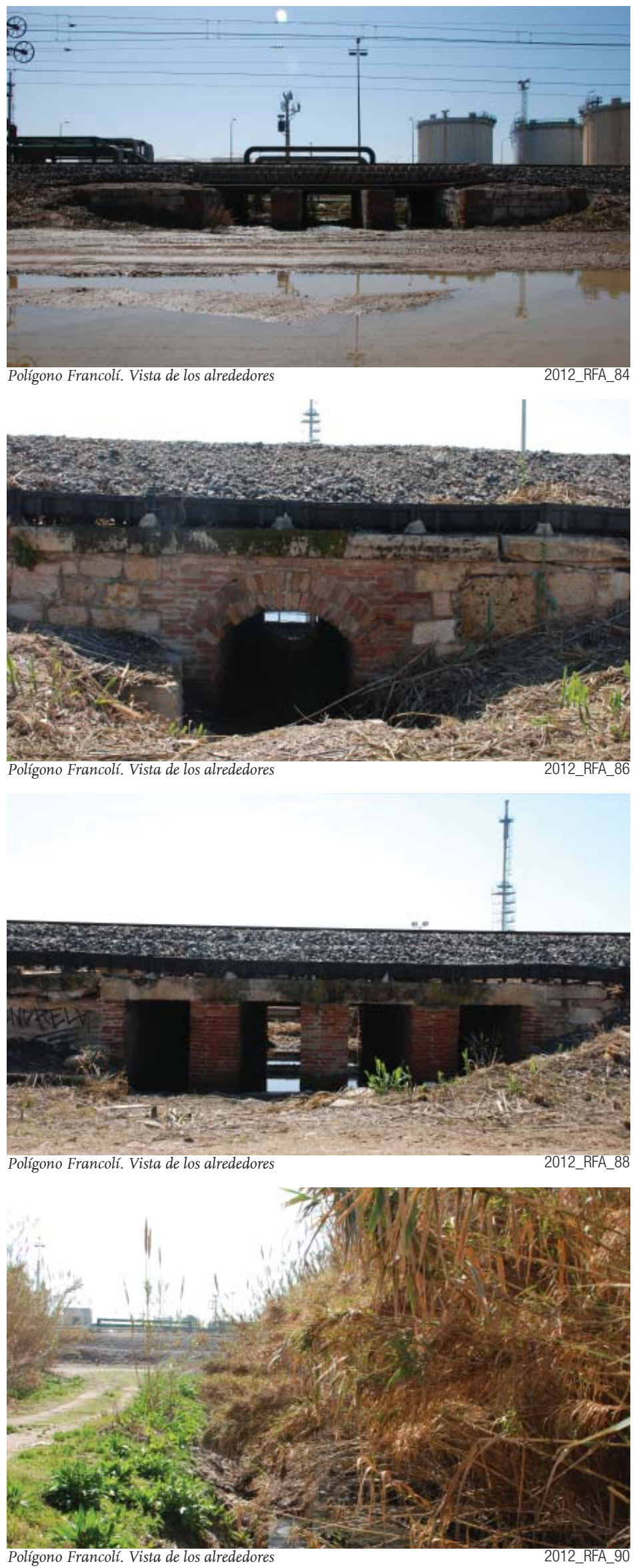

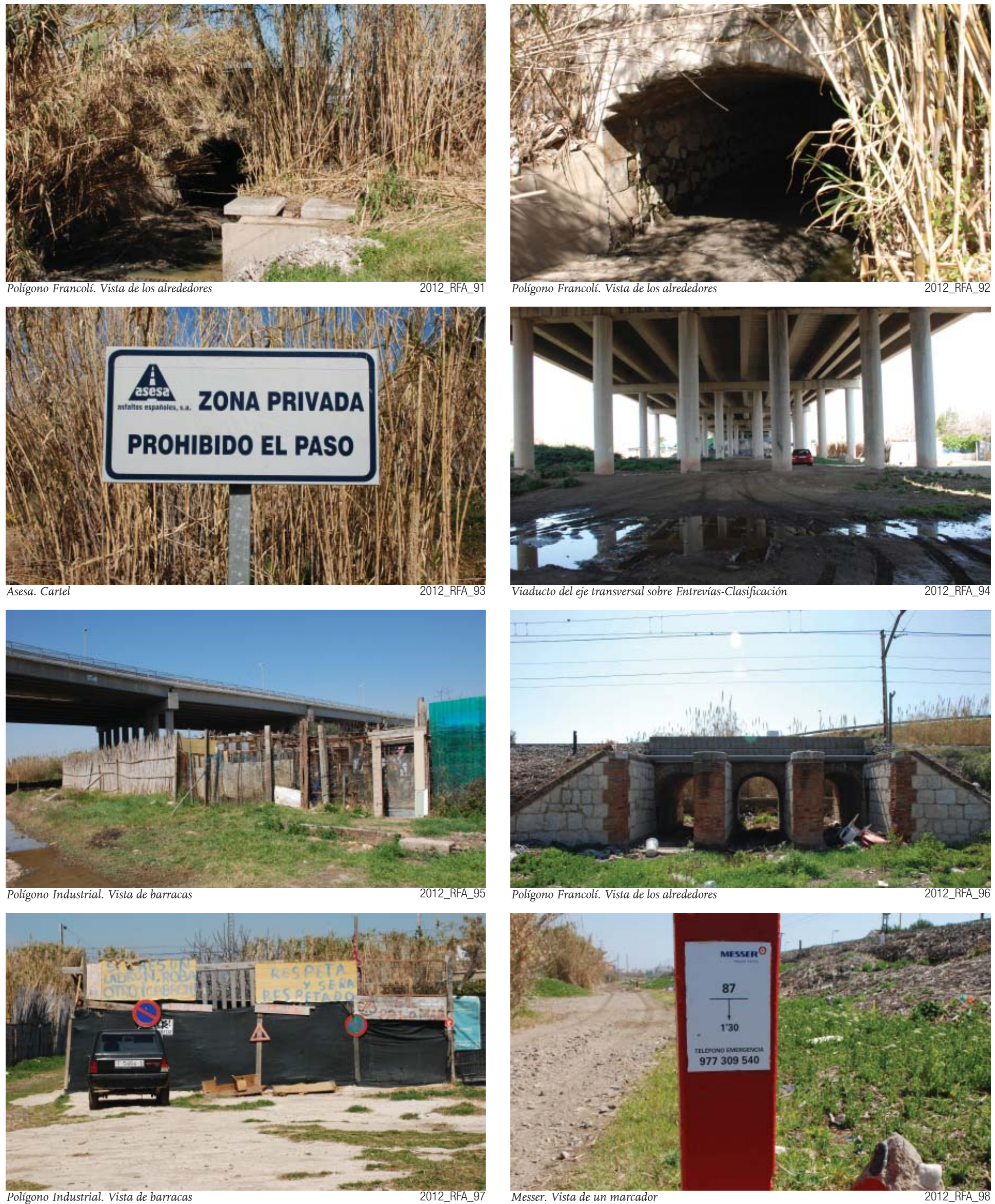

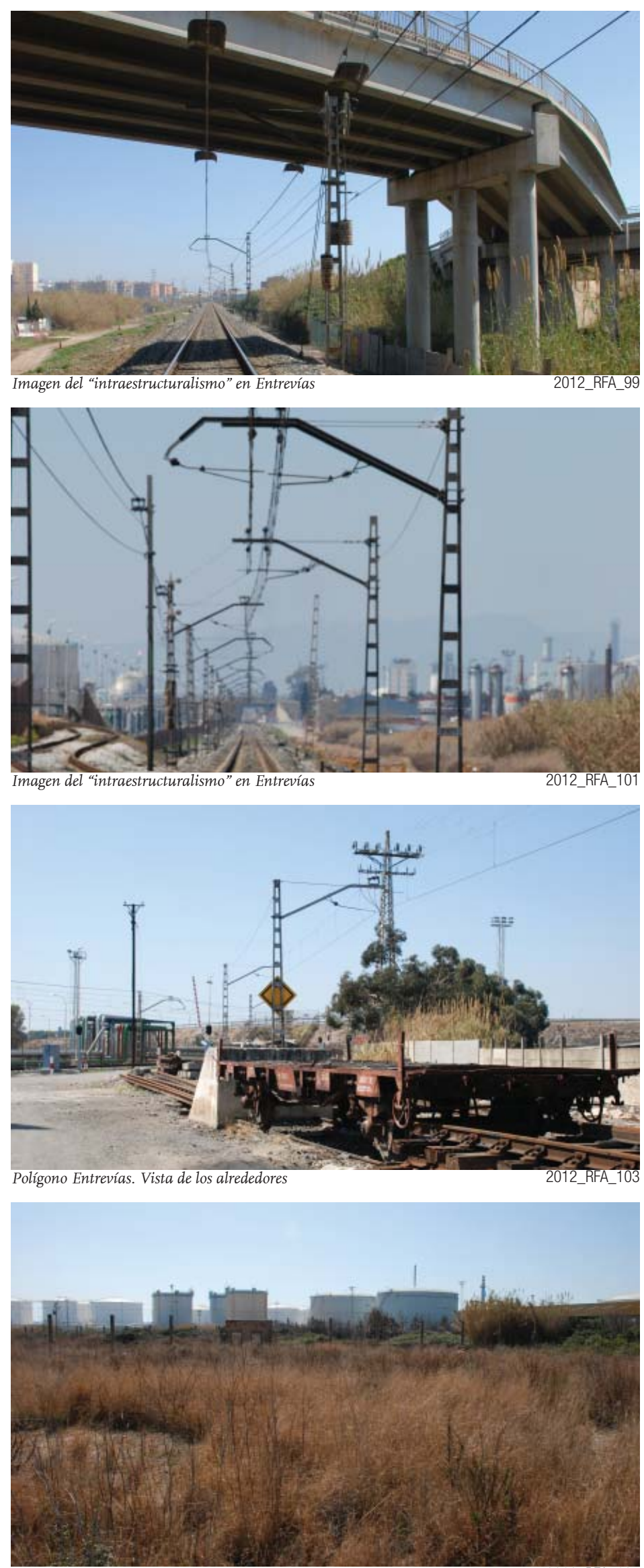
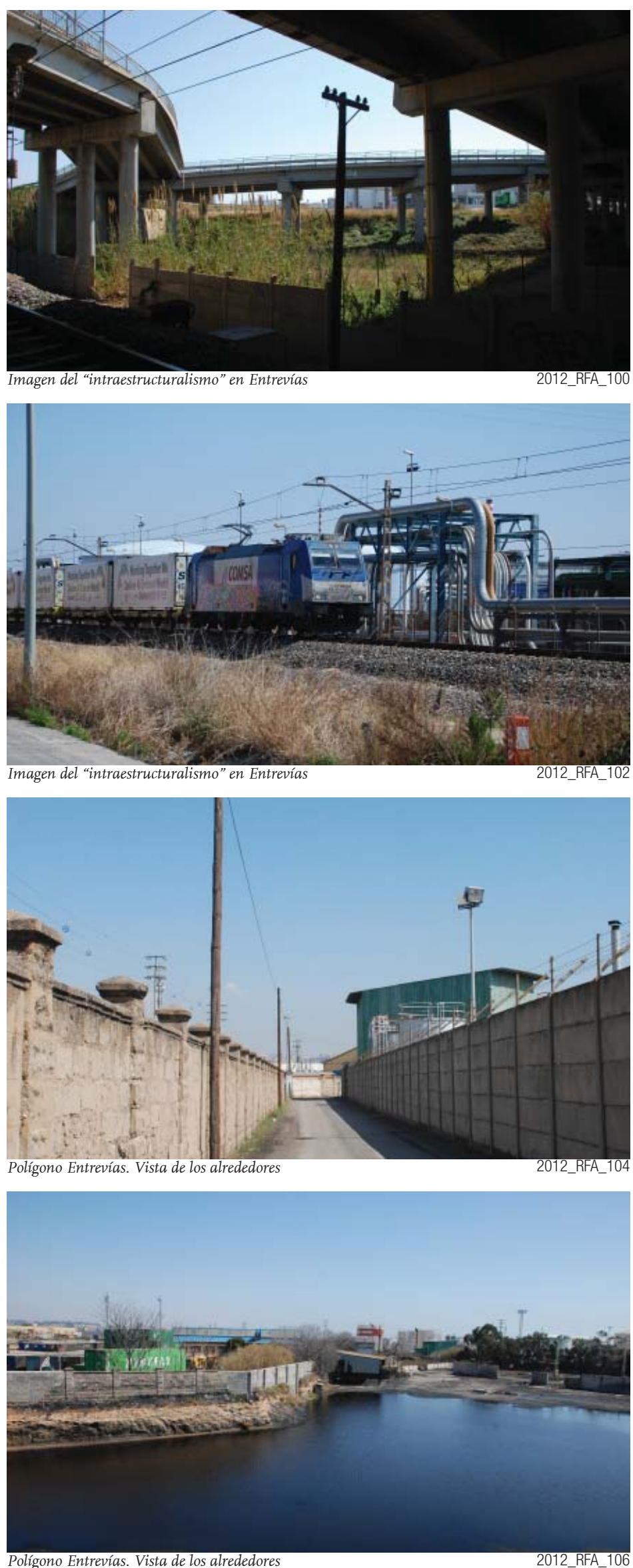


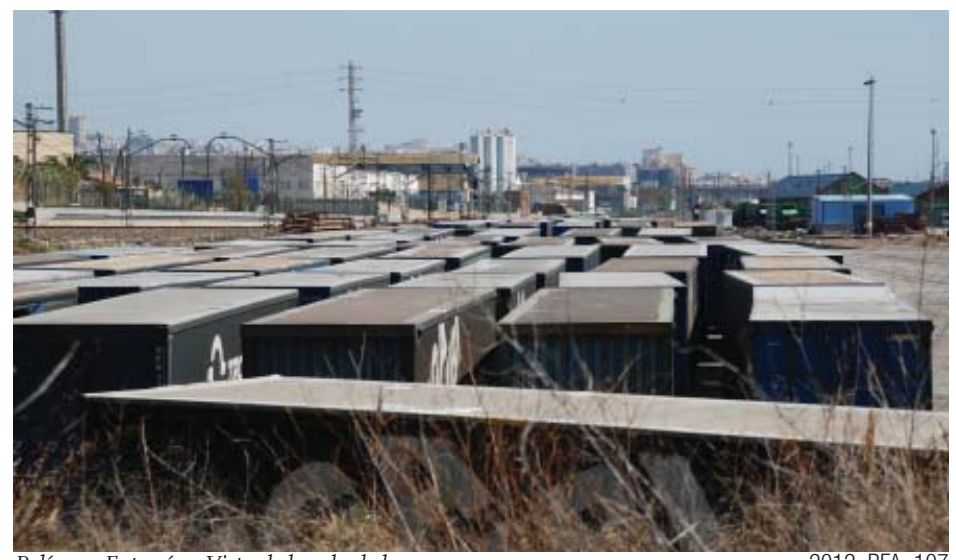

Polígono Entrevías. Vista de los alrededores
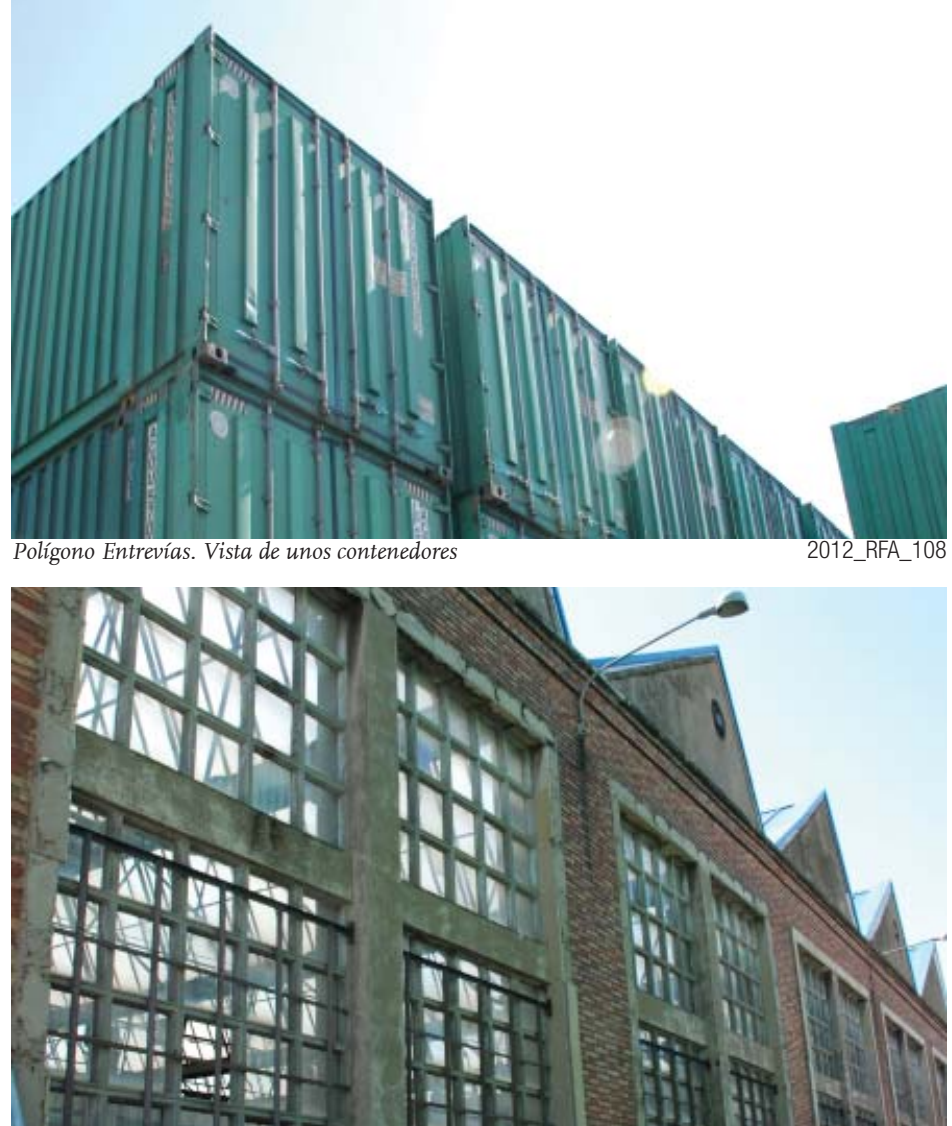

Polígono Entrevías. Vista de las naves
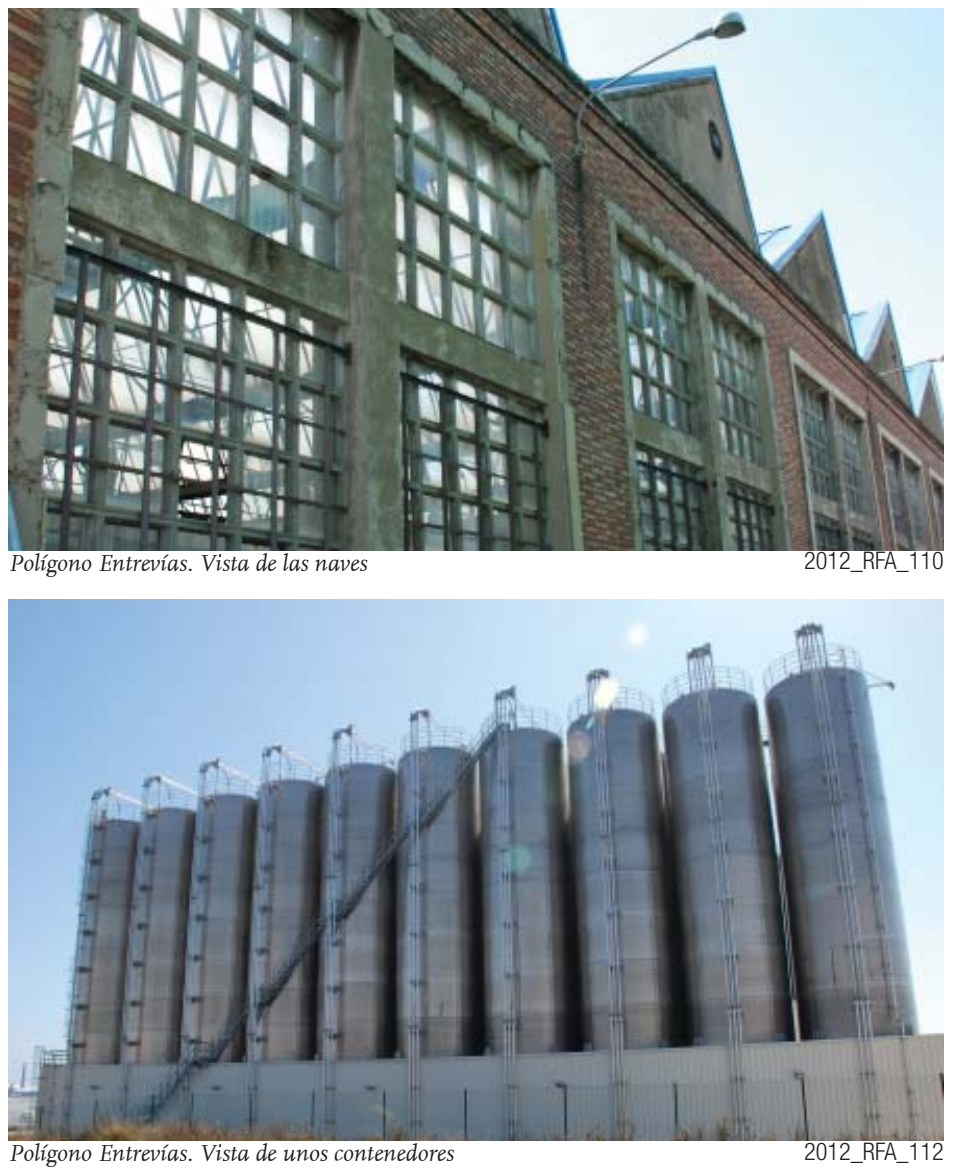
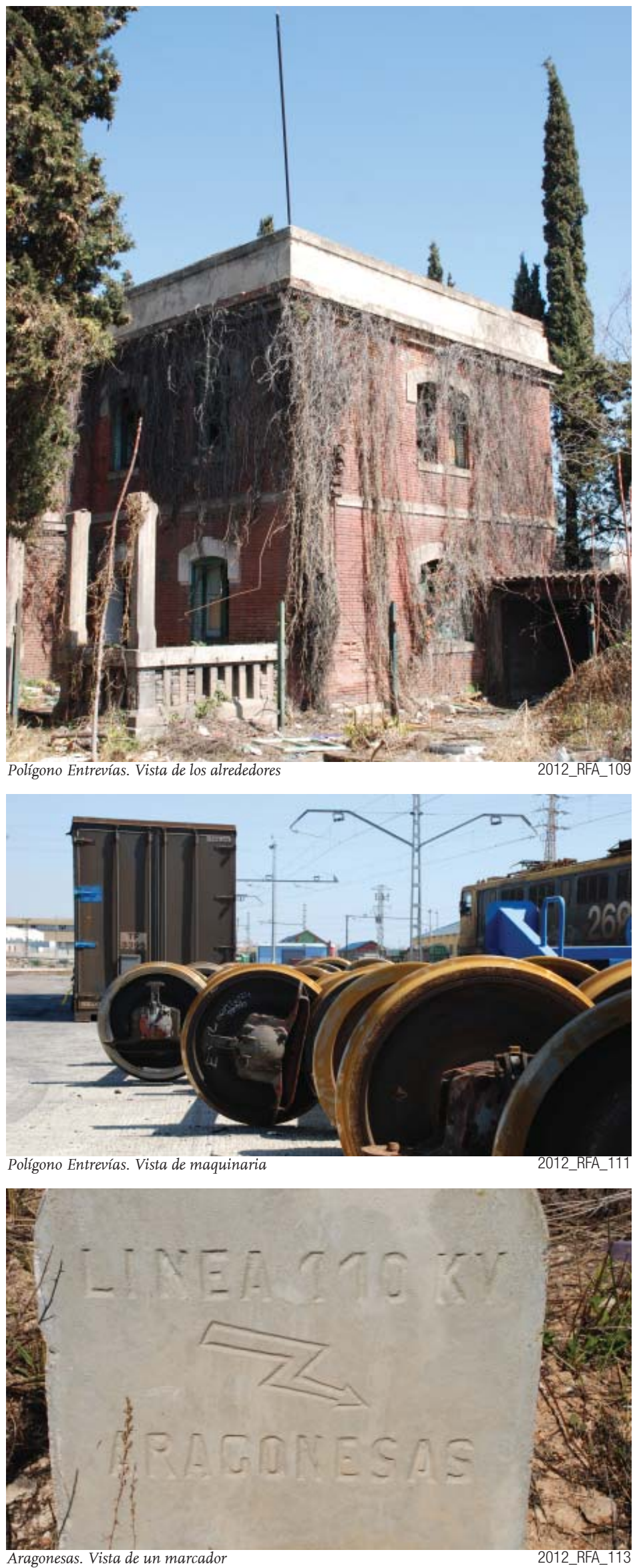

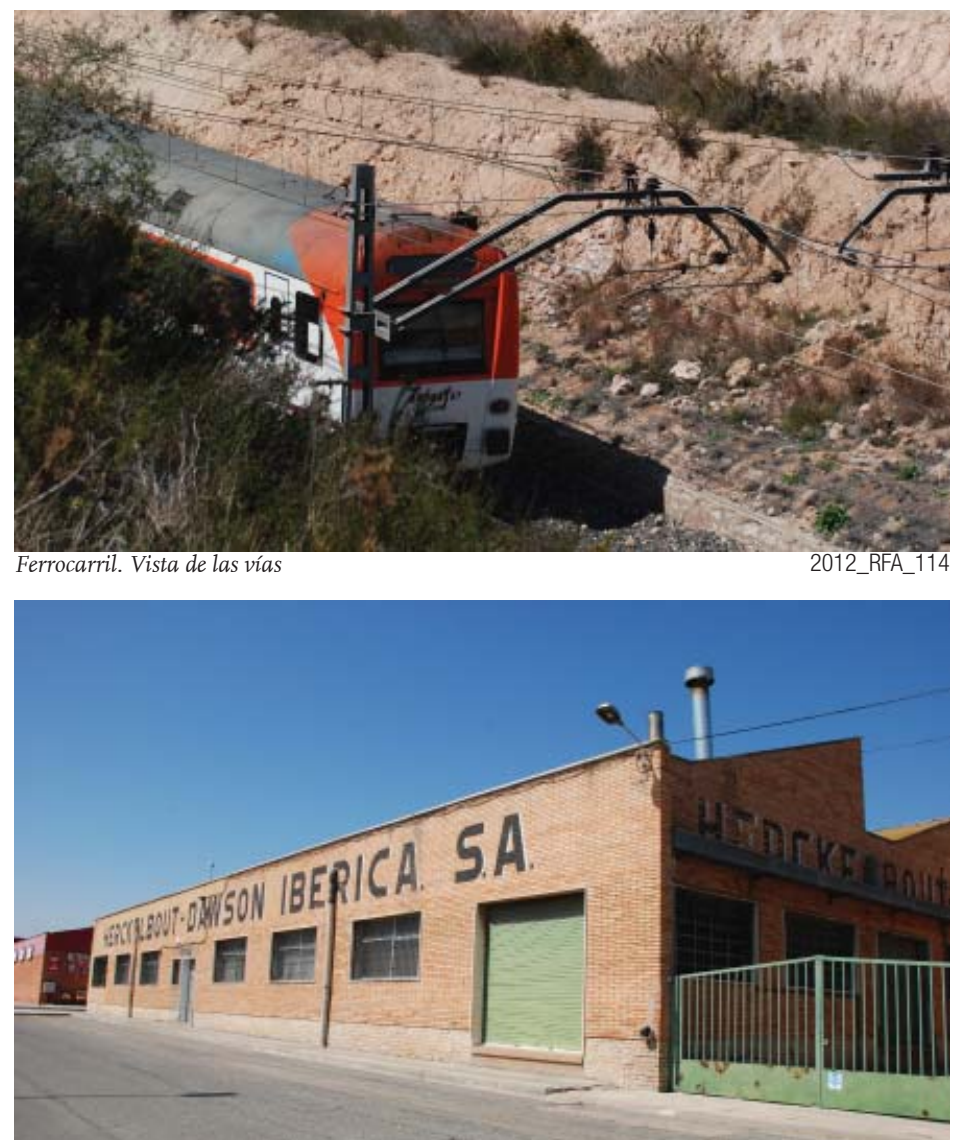

Polígono Entrevías. Vista de las naves

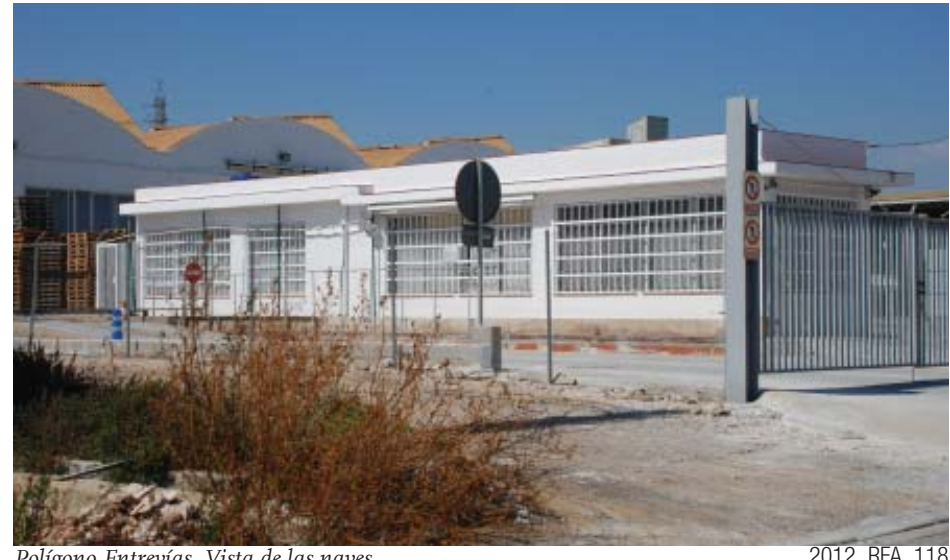

Polígono Entrevías. Vista de las naves

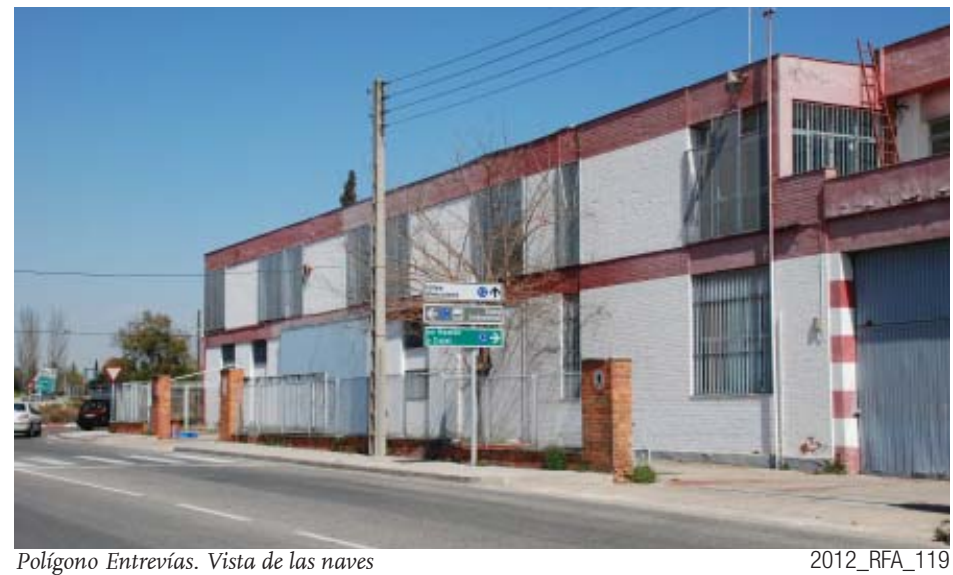

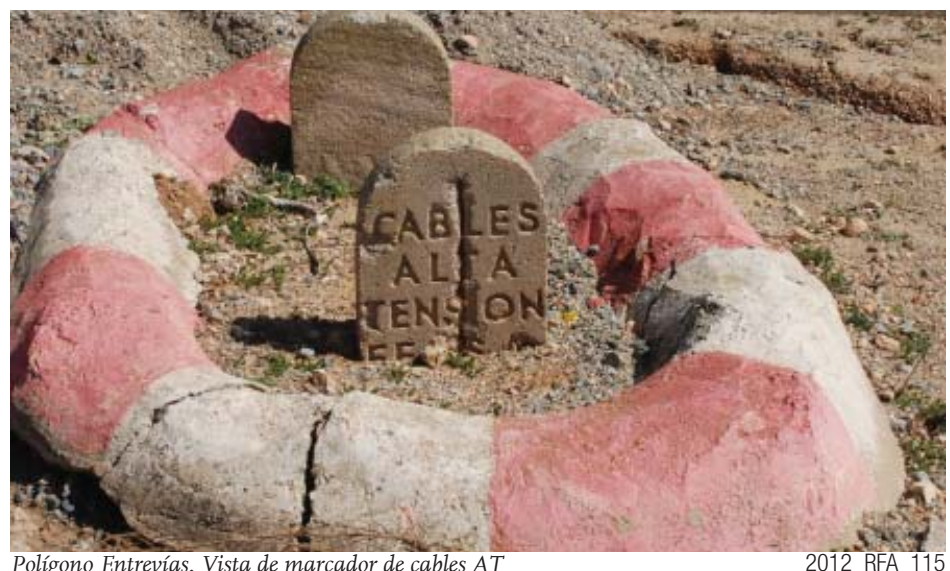

Polígono Entrevías. Vista de marcador de cables AT
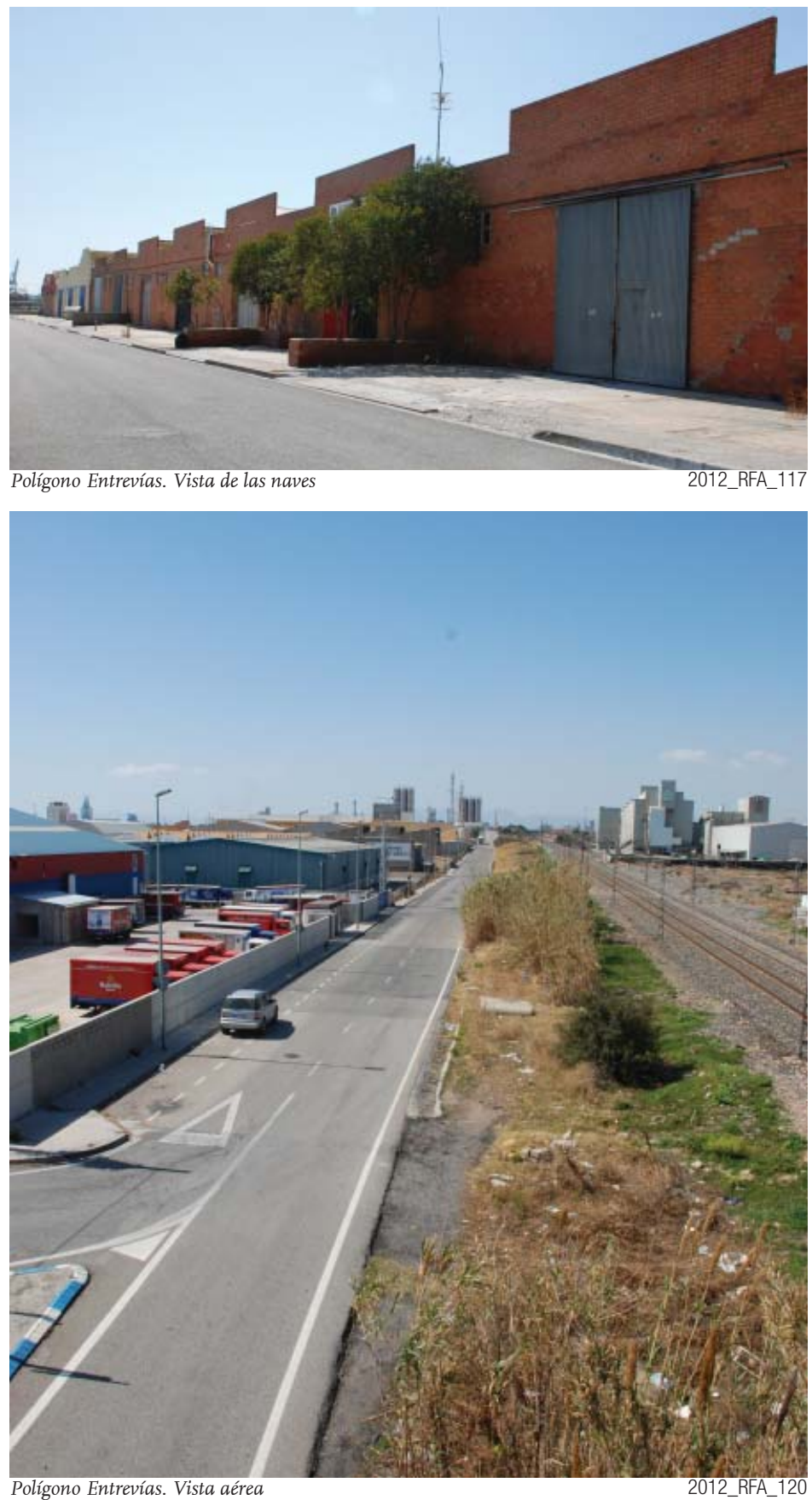

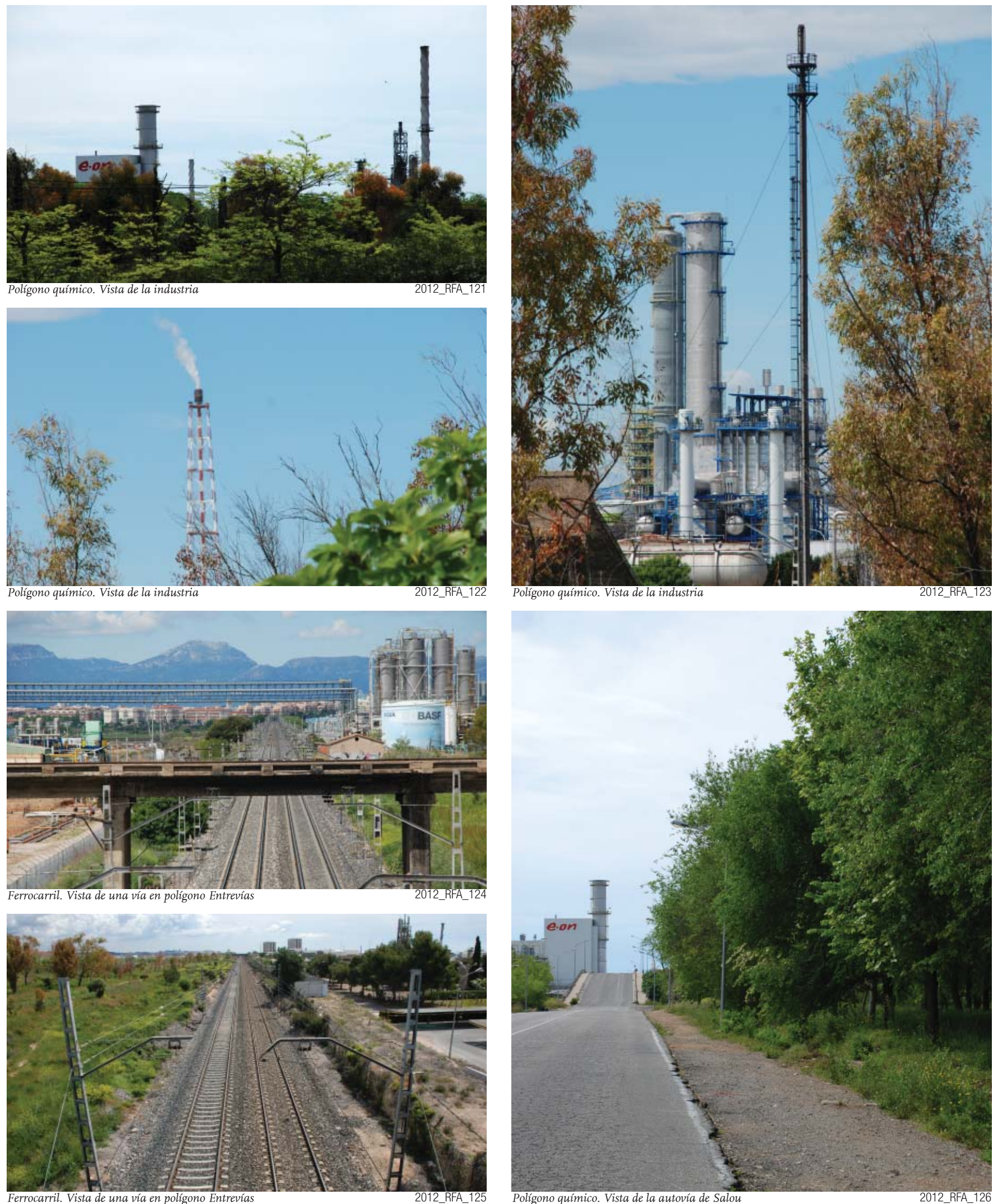

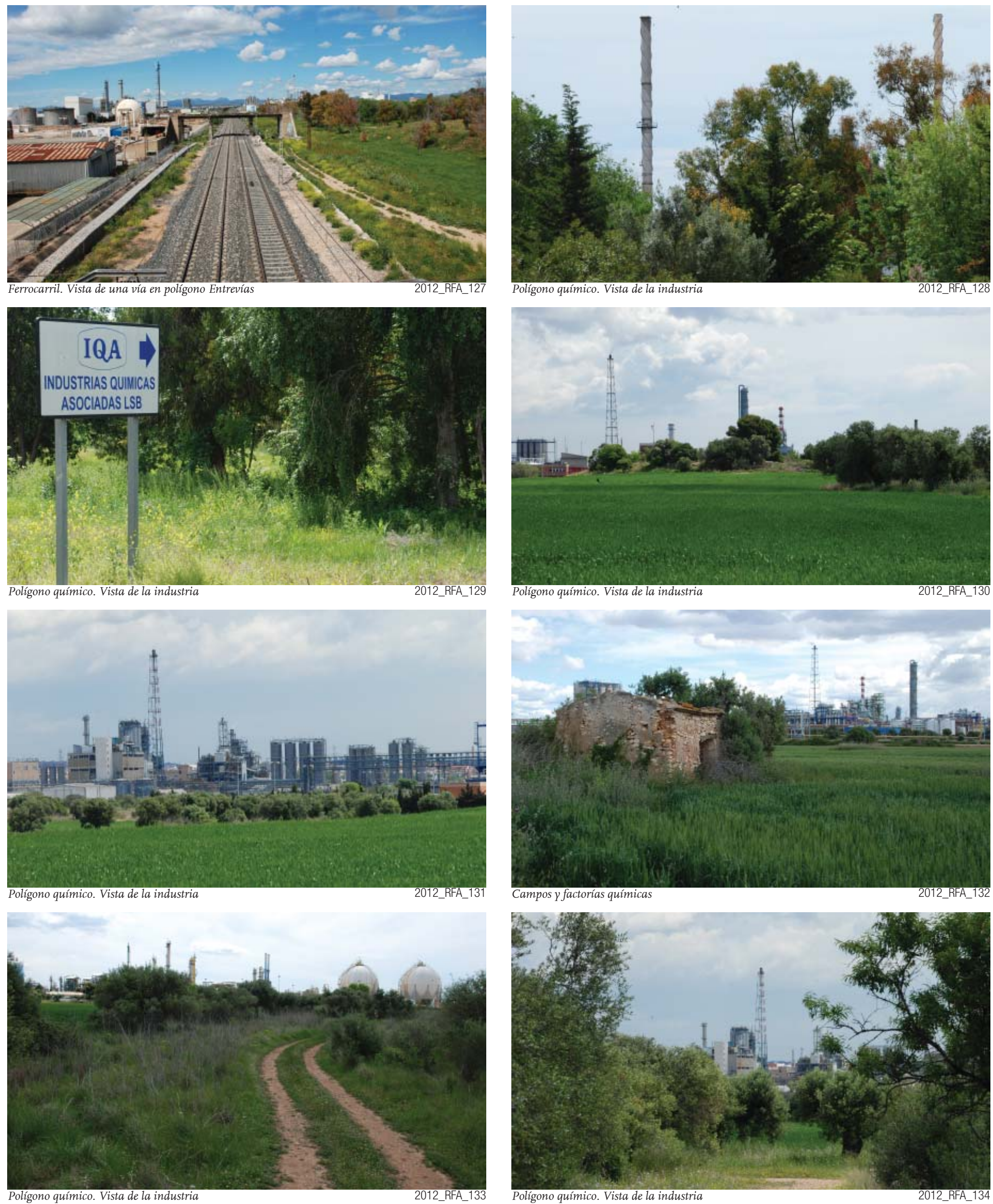

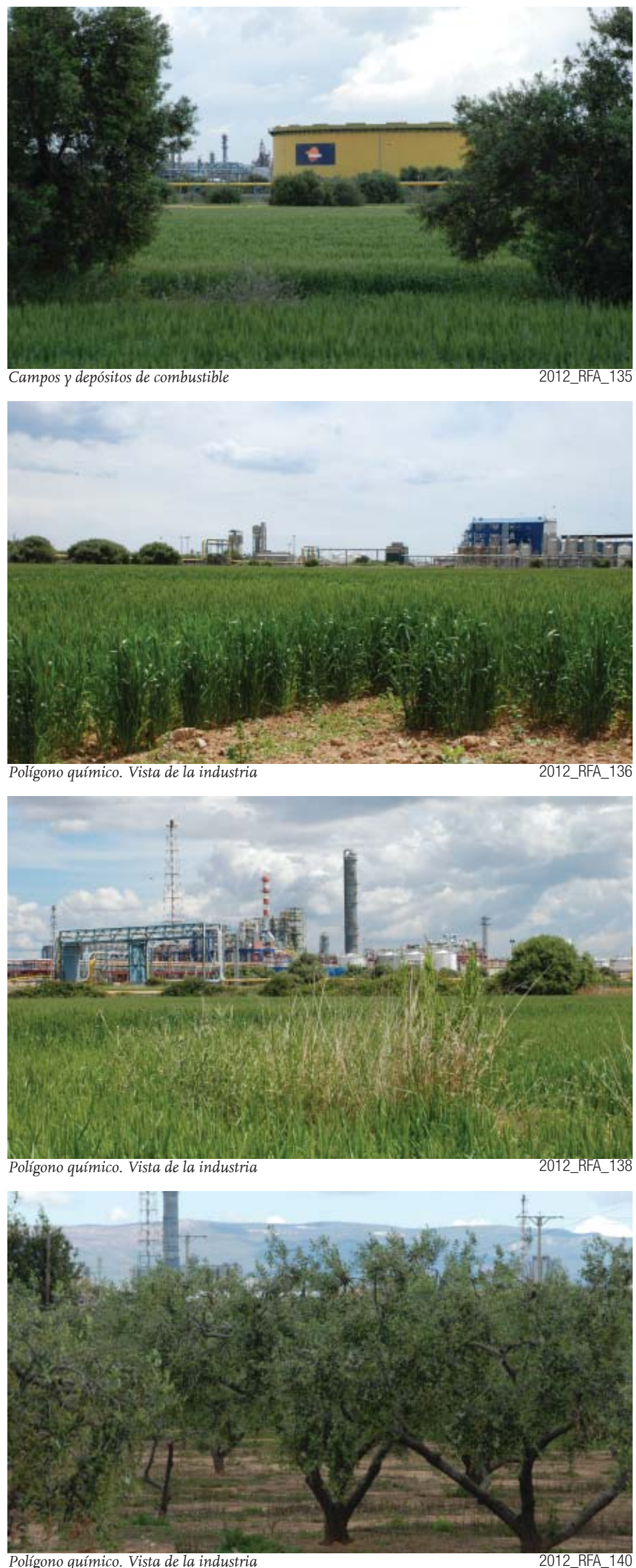
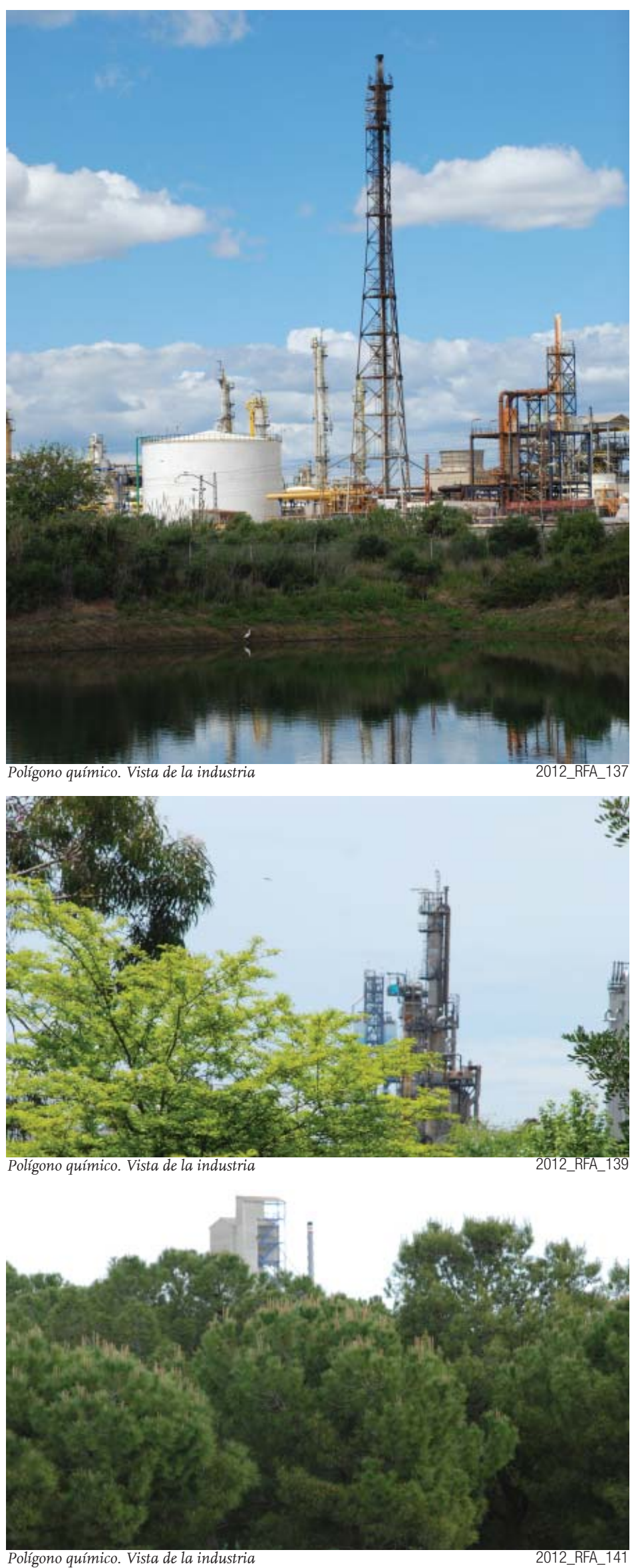

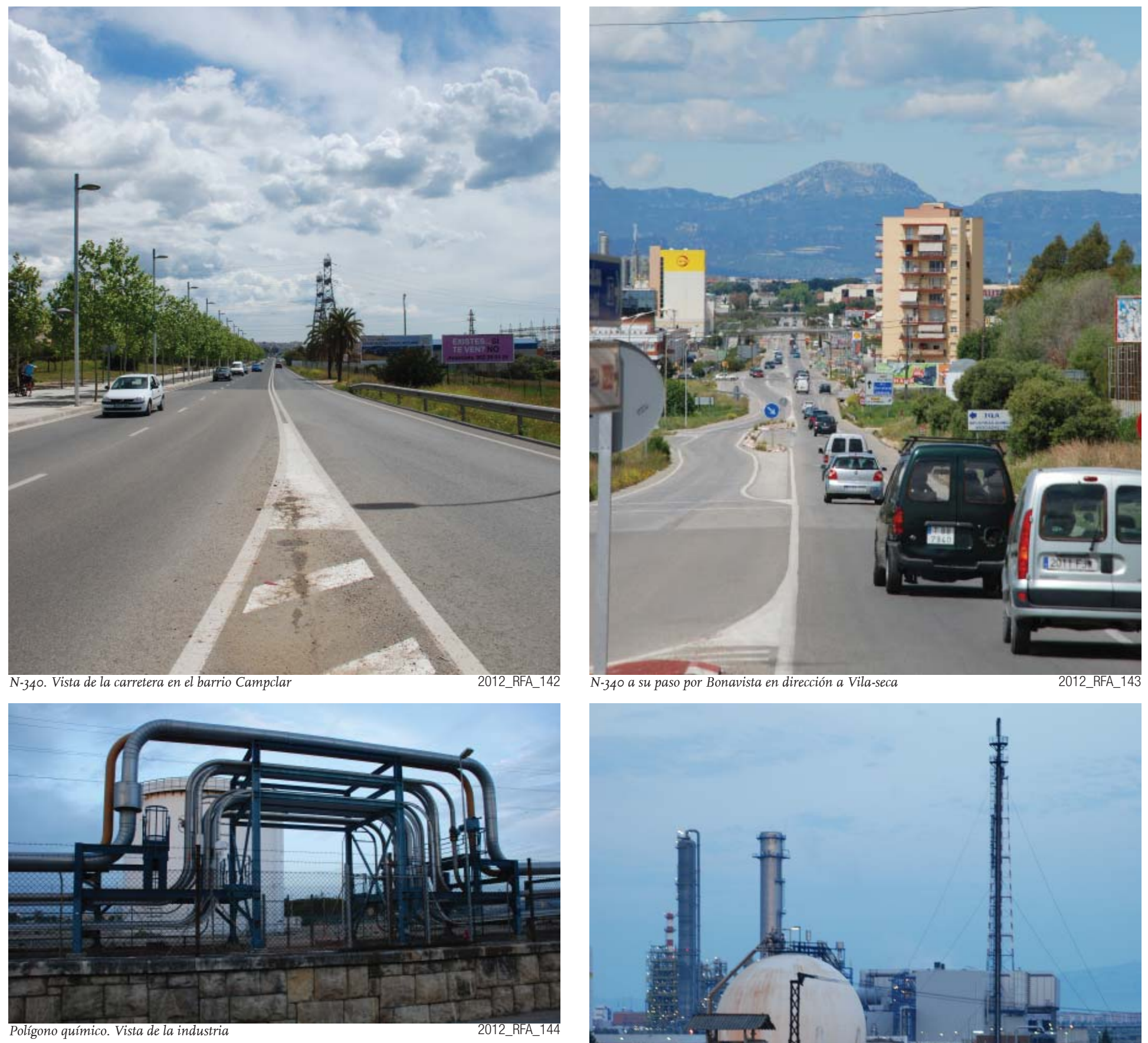

N-340 a su paso por Bonavista en dirección a Vila-seca

2012_RFA_143

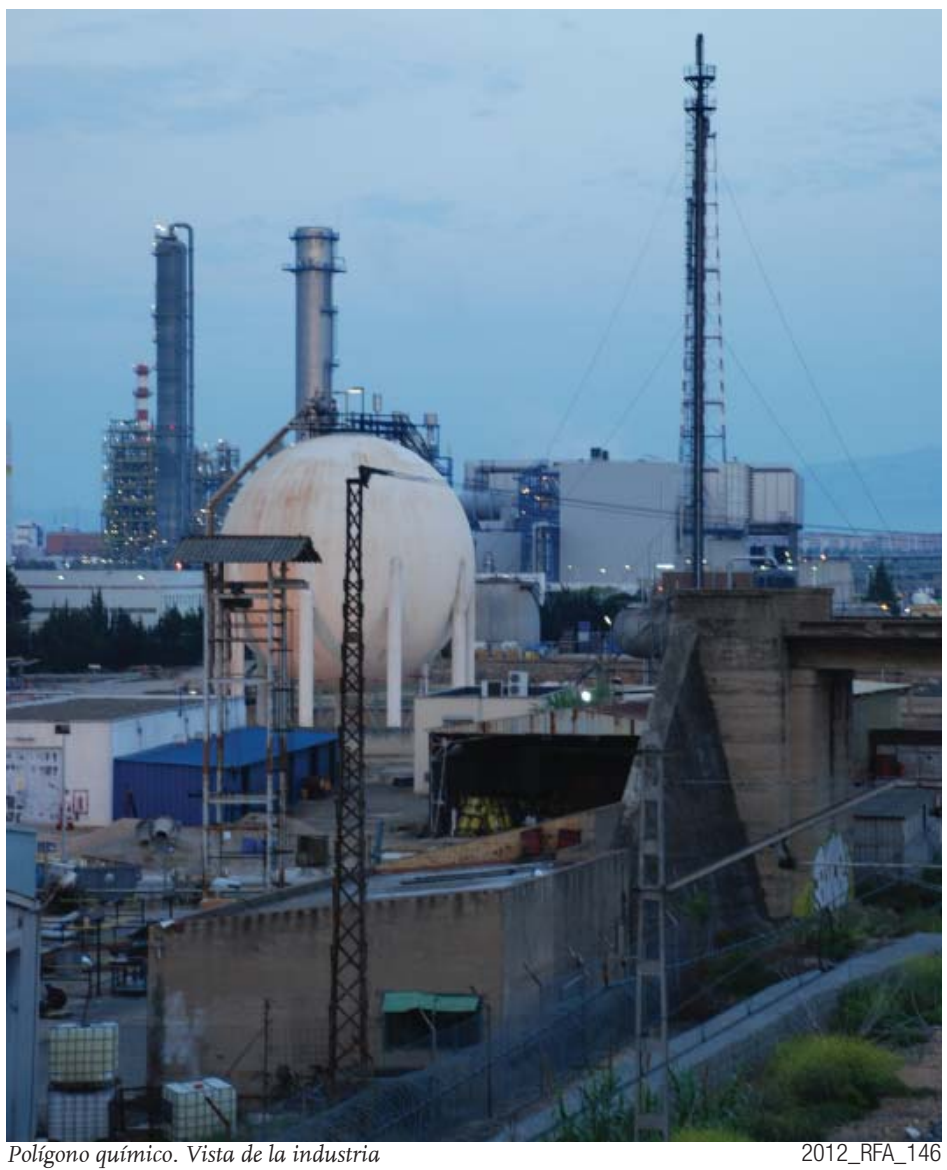



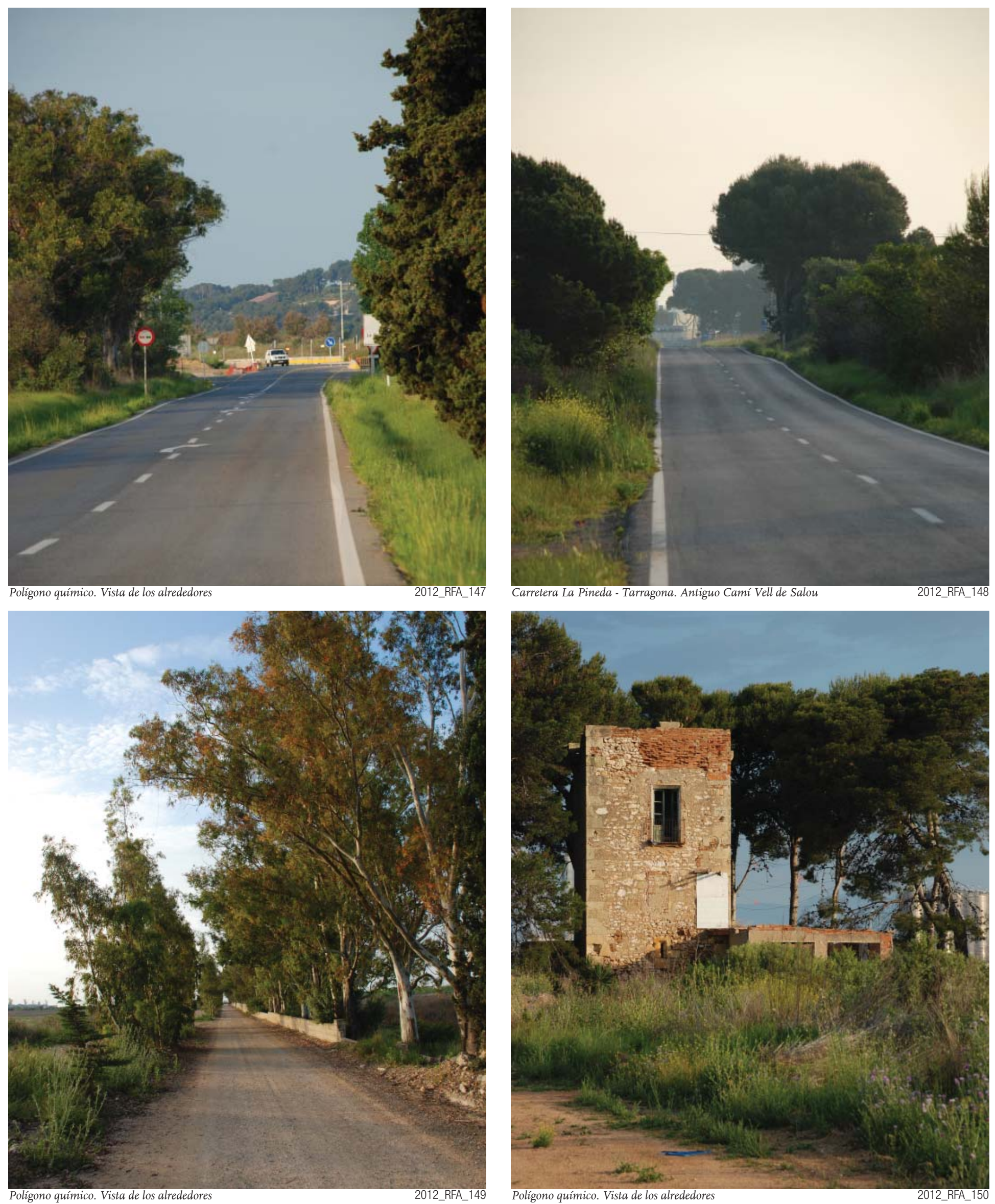

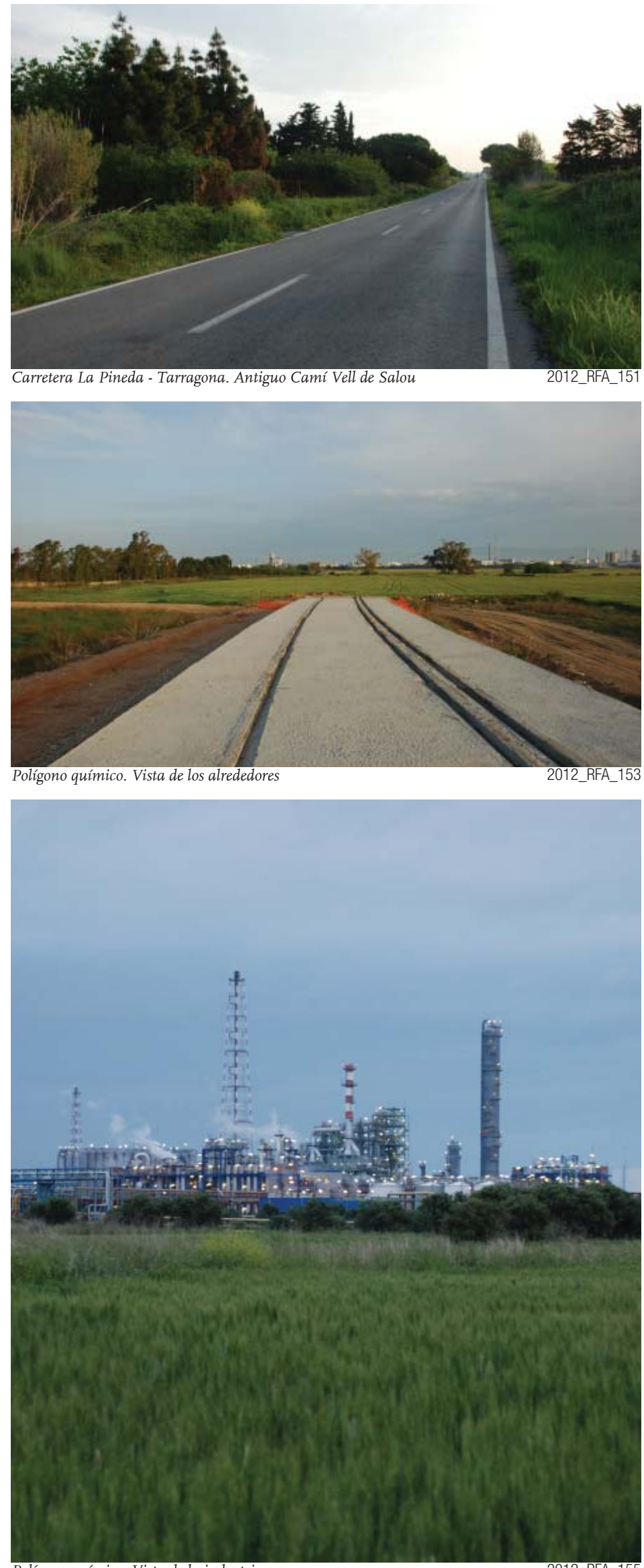

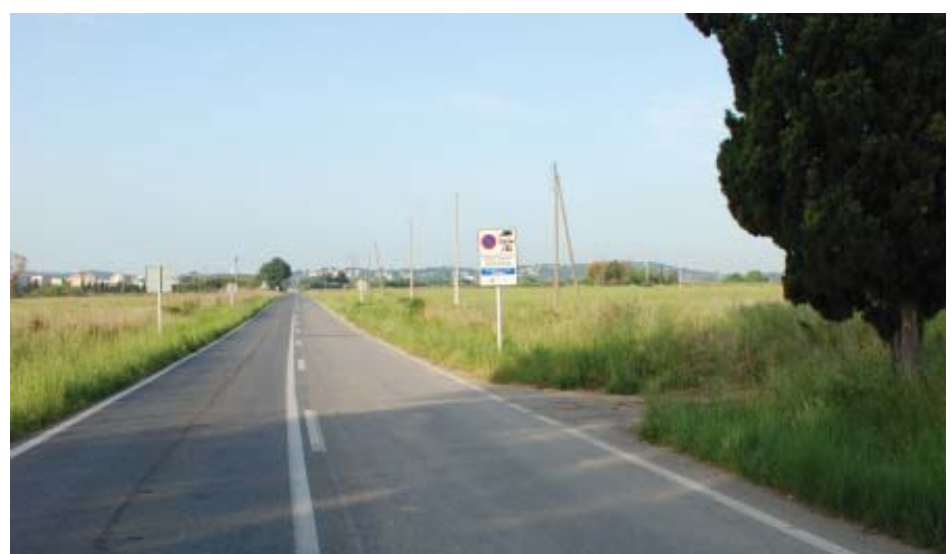

Polígono químico. Vista de los alrededores

2012_RFA_152
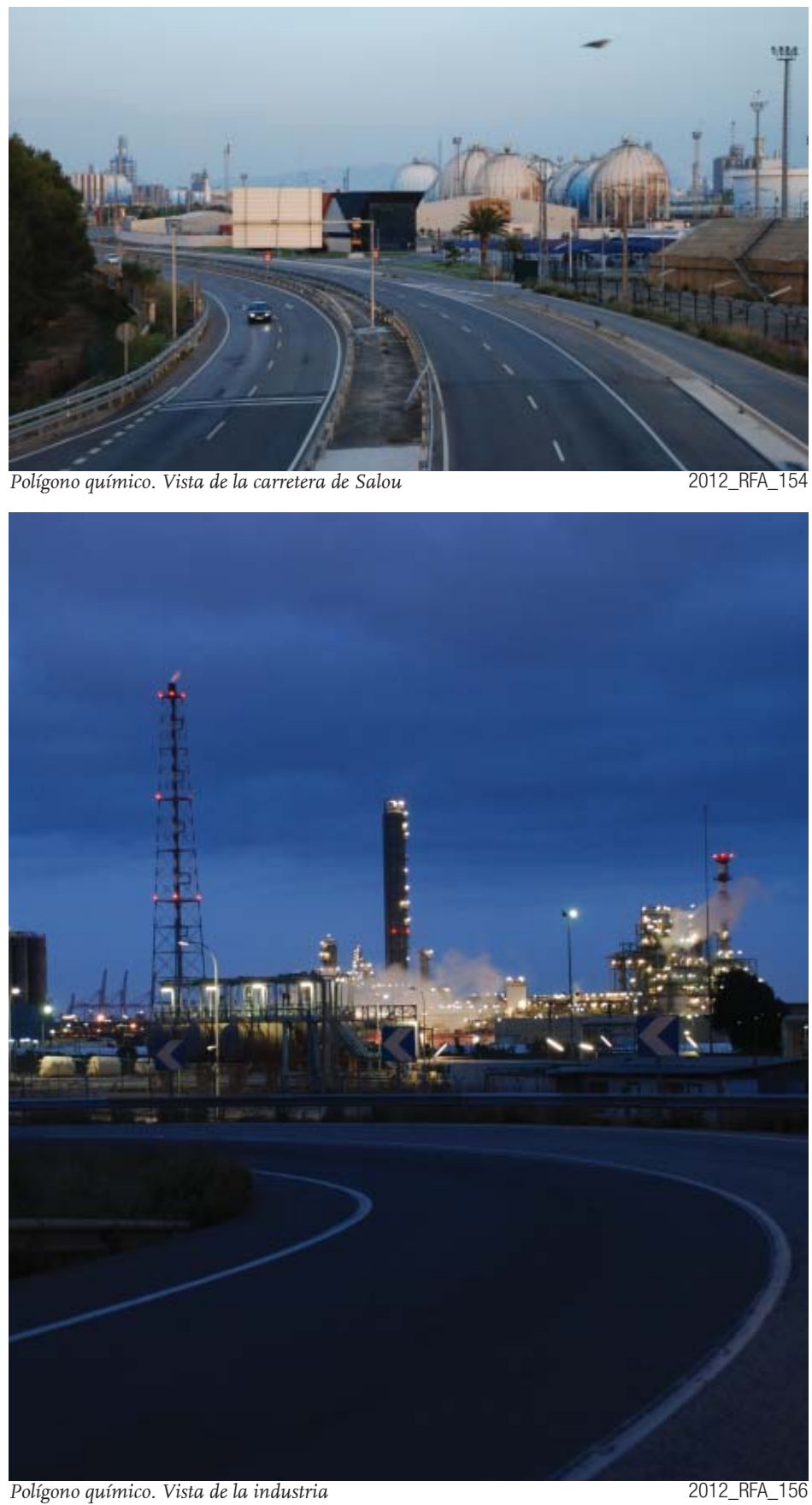

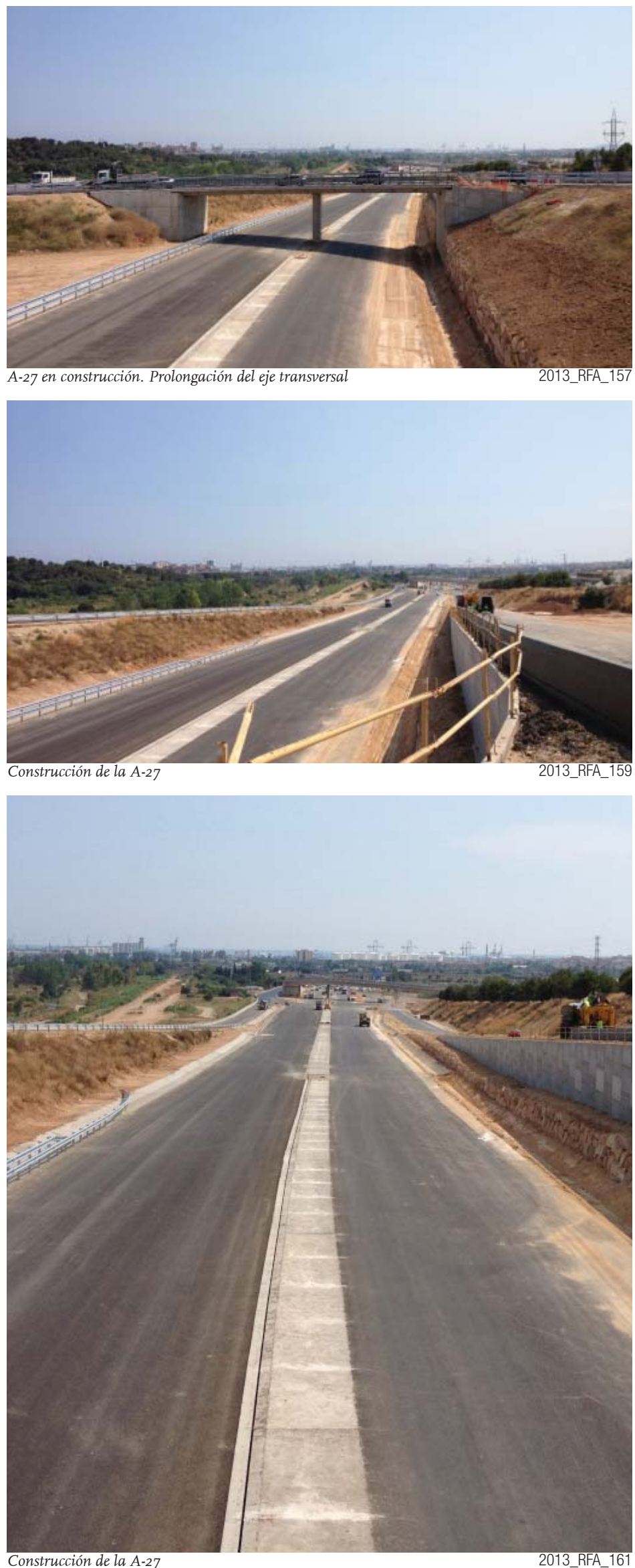

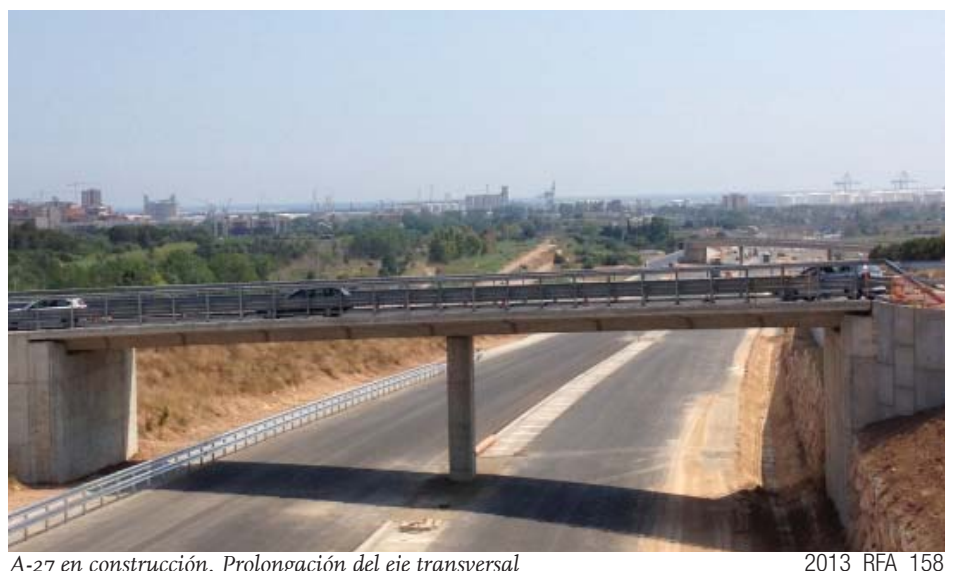

A-27 en construcción. Prolongación del eje transversal
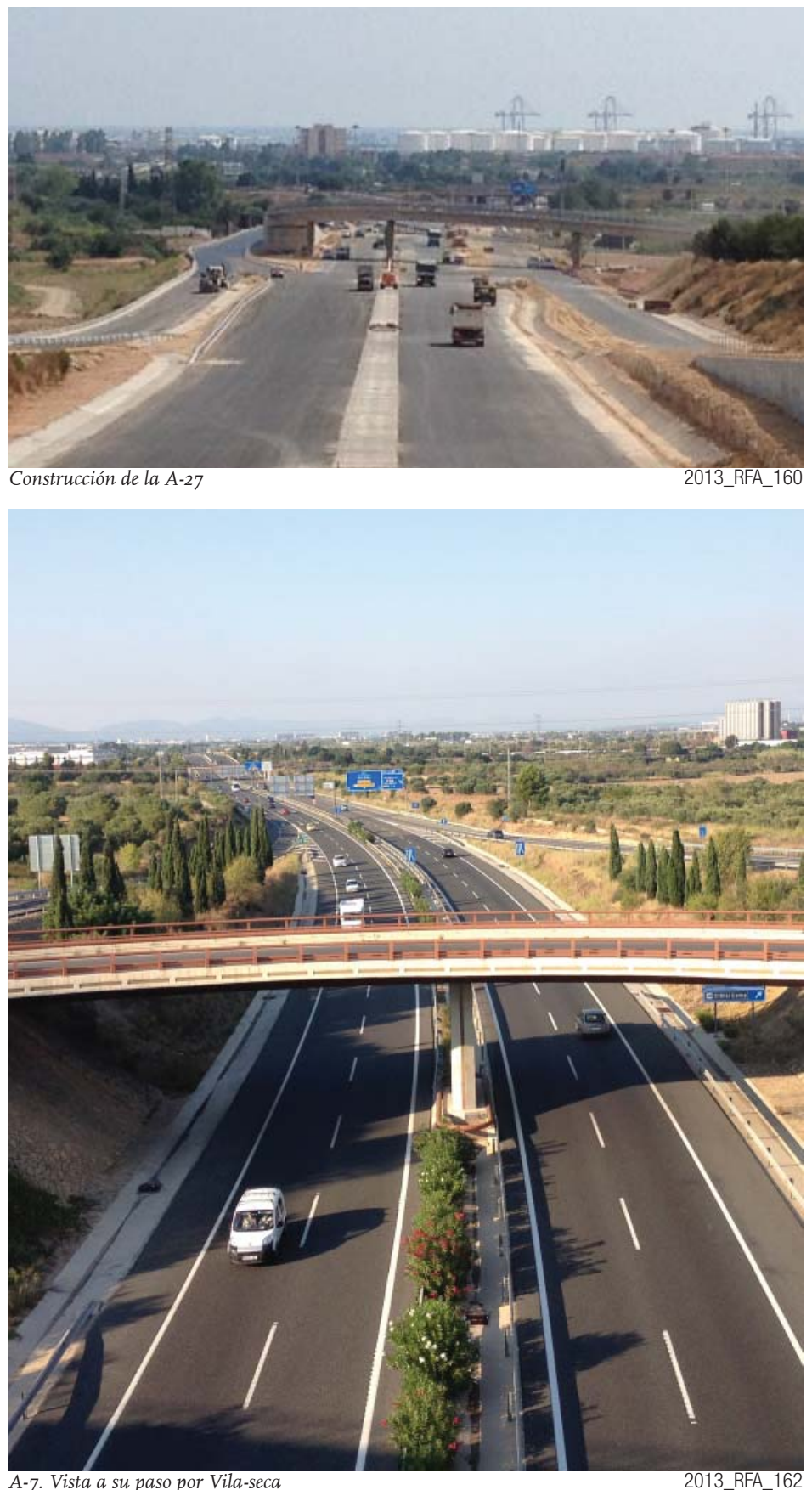

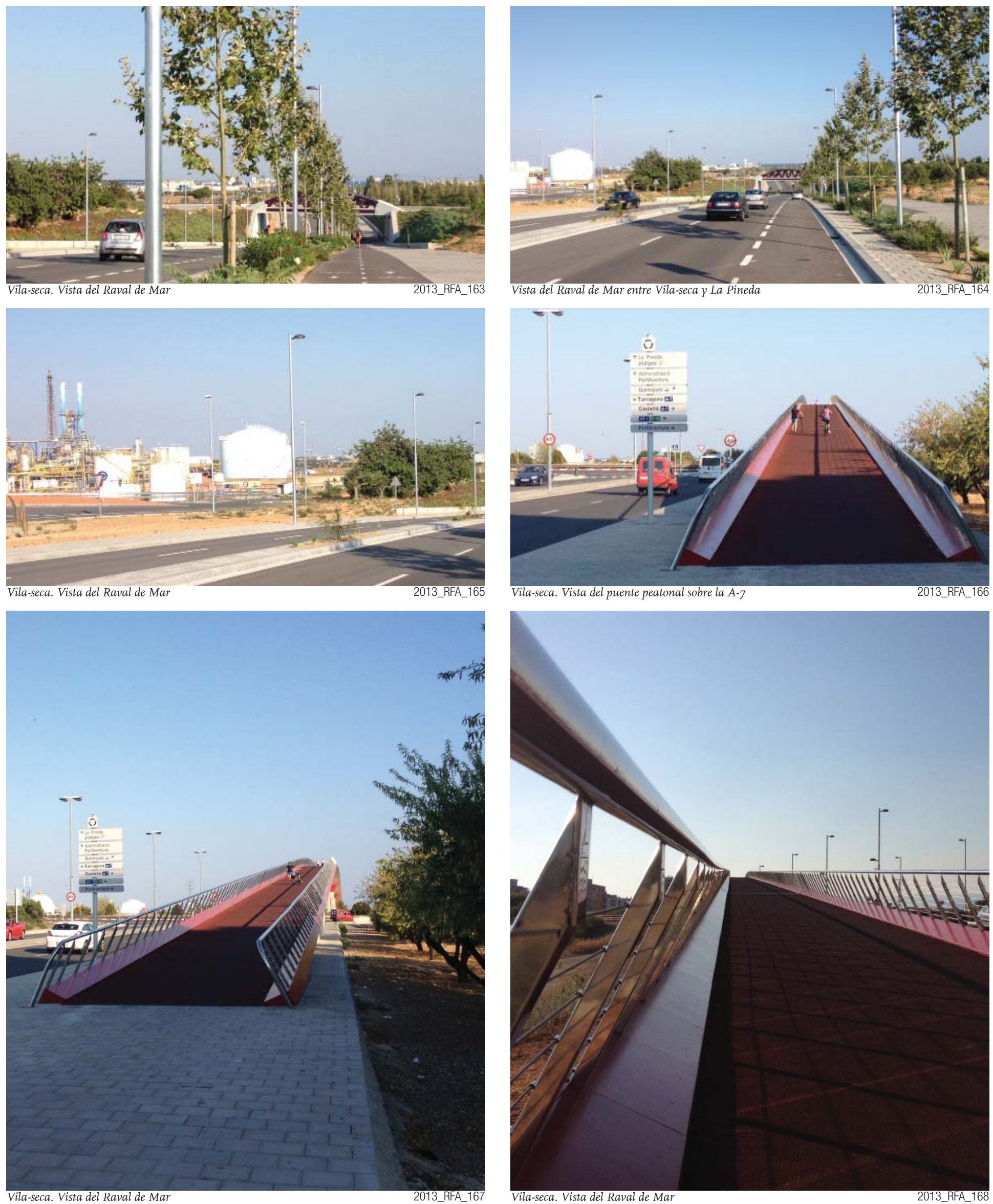


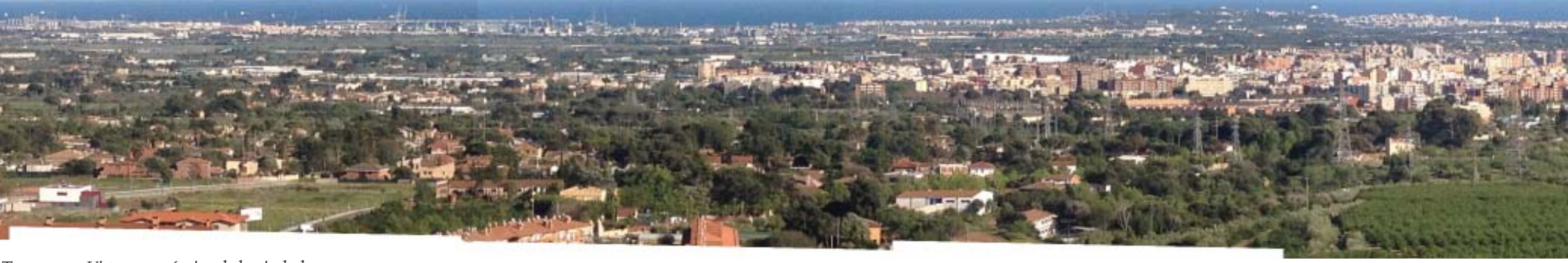

Tarragona. Vista panorámica de la ciudad
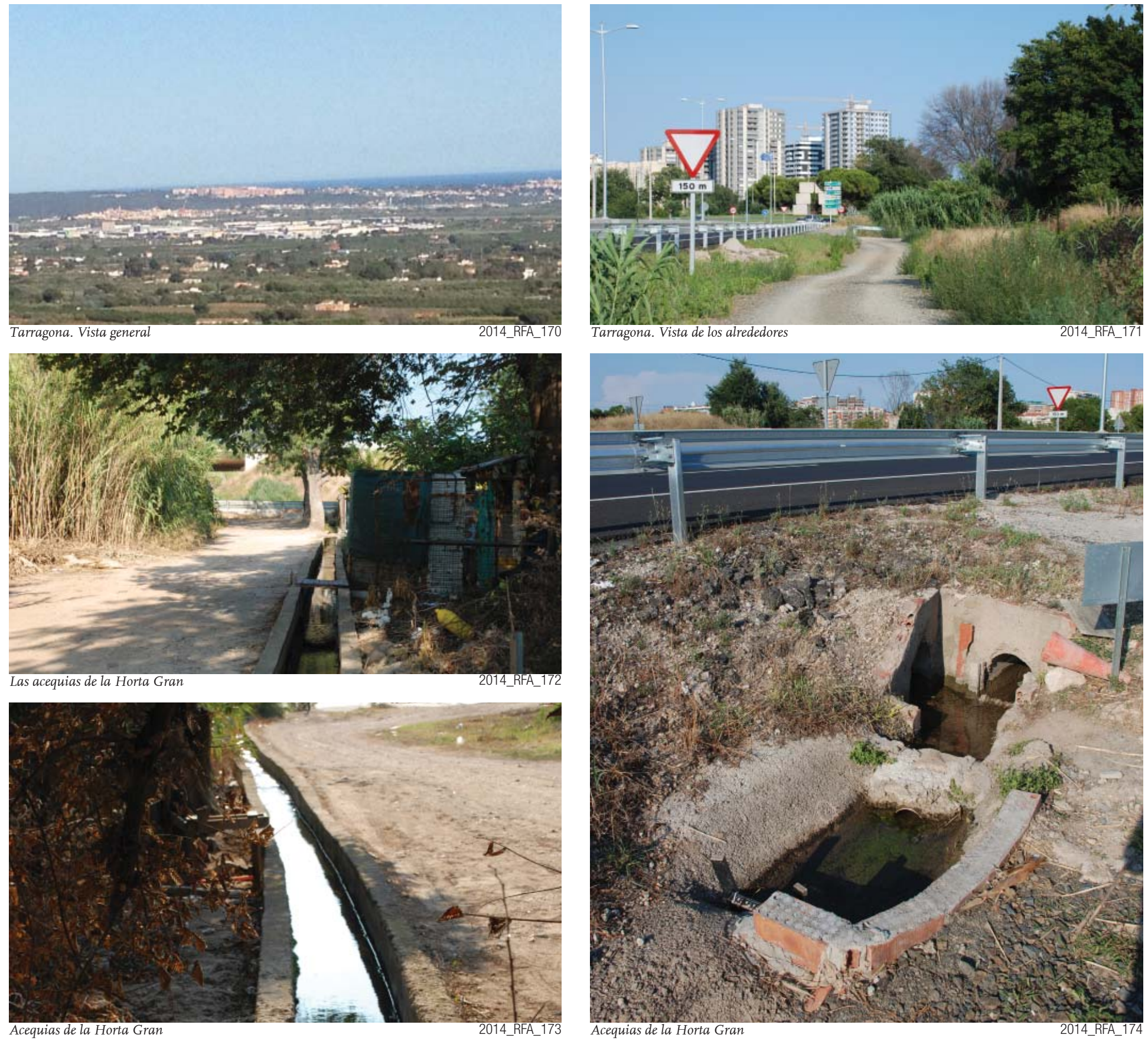


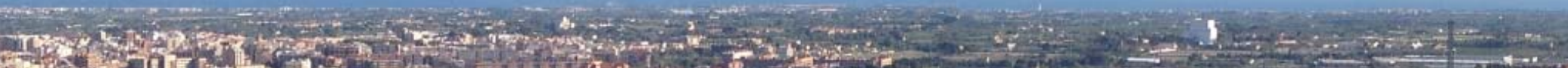

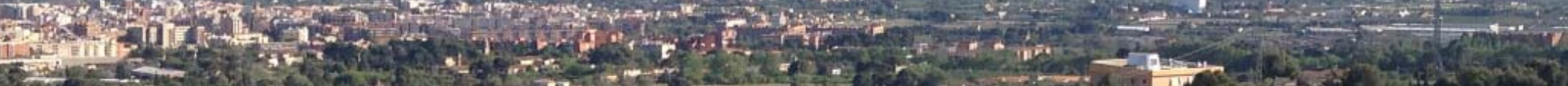
(7)

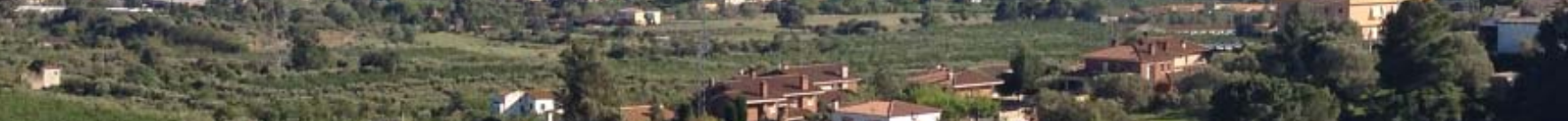

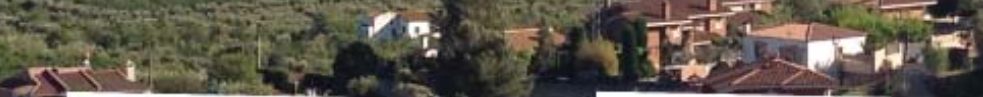
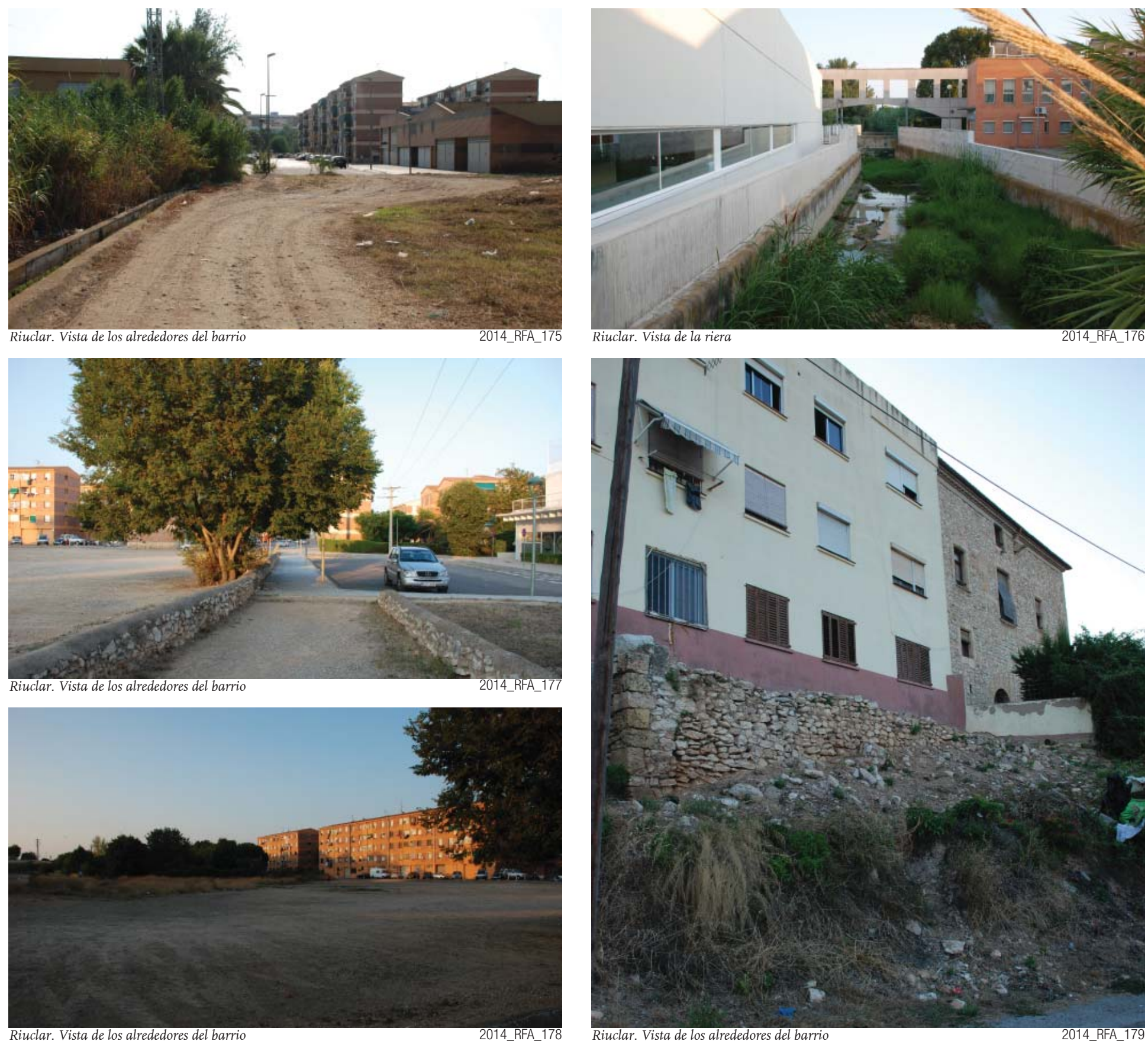

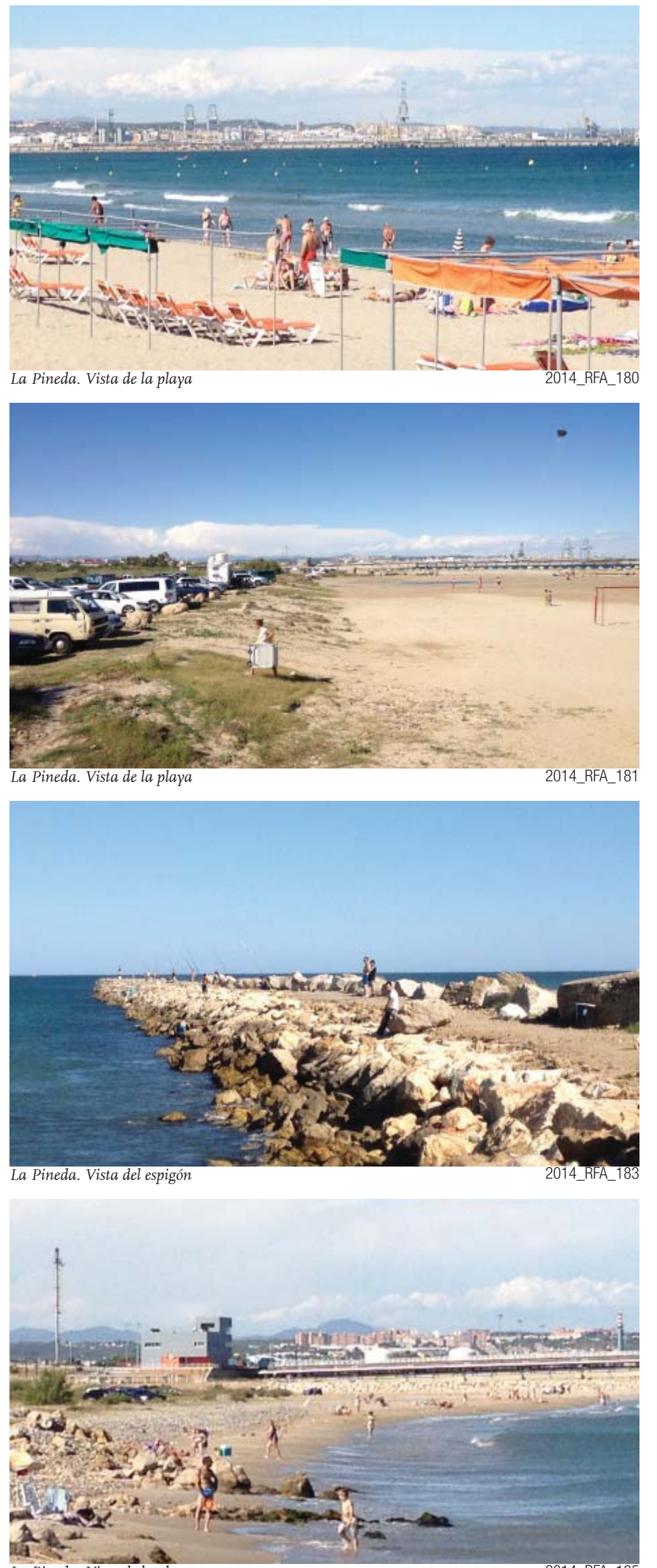
La Pineda. Vista de la playa
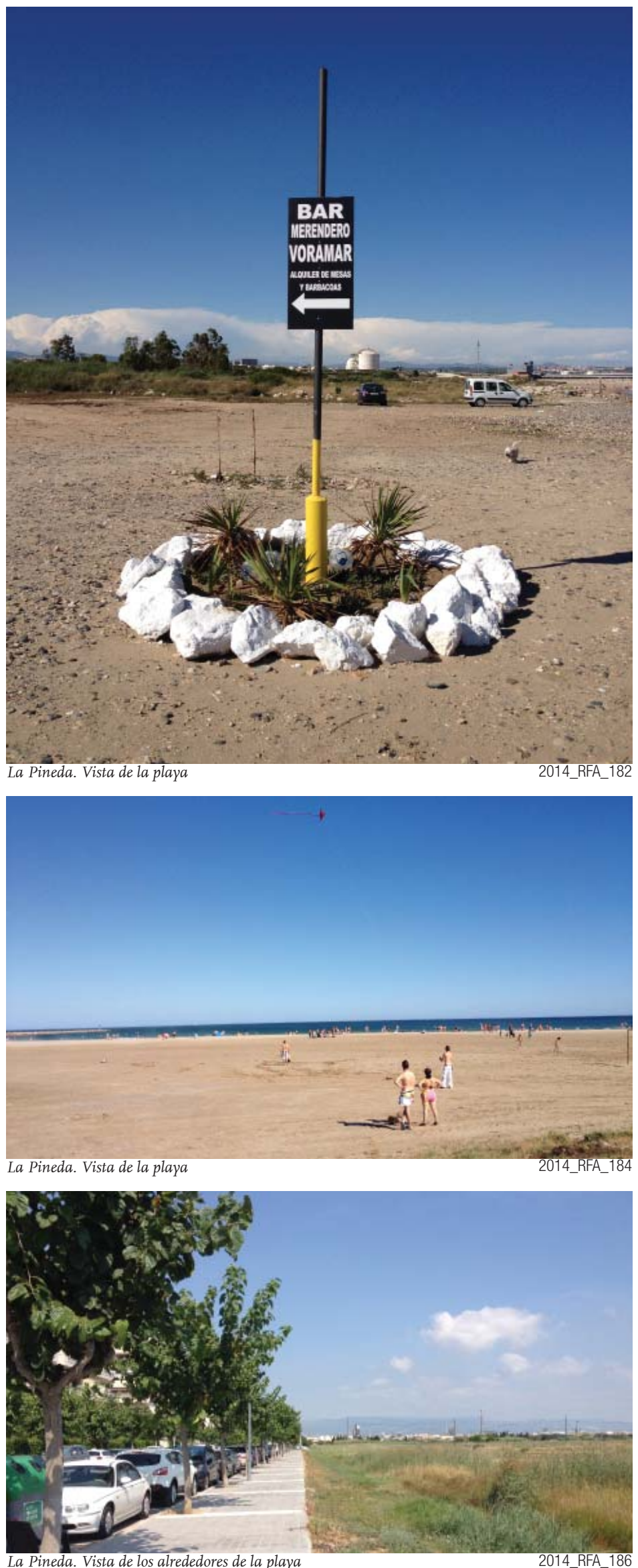

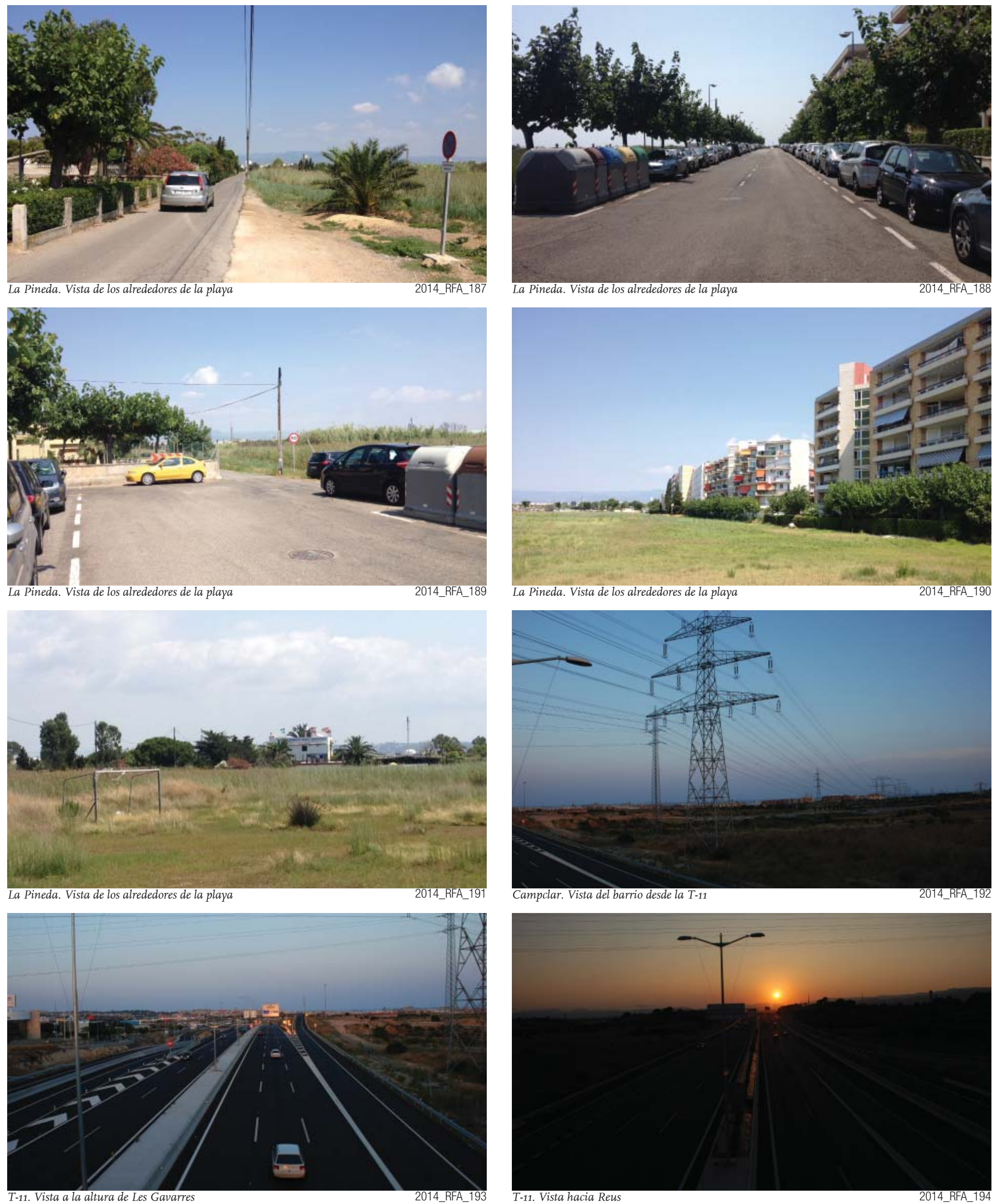
"El significado de la ciudad [territorio], desde esta perspectiva, es una pregunta que no puede responderse con la razón técnico-constructiva”. De ahí que “...no sea la racionalidad lo que permite comprender algunos fenómenos de la ciudad [territorio], sino que son más bien algunos fenómenos puestos de manifiesto por la ciudad [territorio] lo que nos permite construir una simulación de racionalidad y el sueño de un control imposible."

La invención de Caín, de Félix de Azúa, I999, que a la vez cita a La Forme d’une Ville, de Julián Gracq, José Corti, 1985.

\section{DOCUMENTACIÓN \\ GRÁFICA \\ ELABORADA}

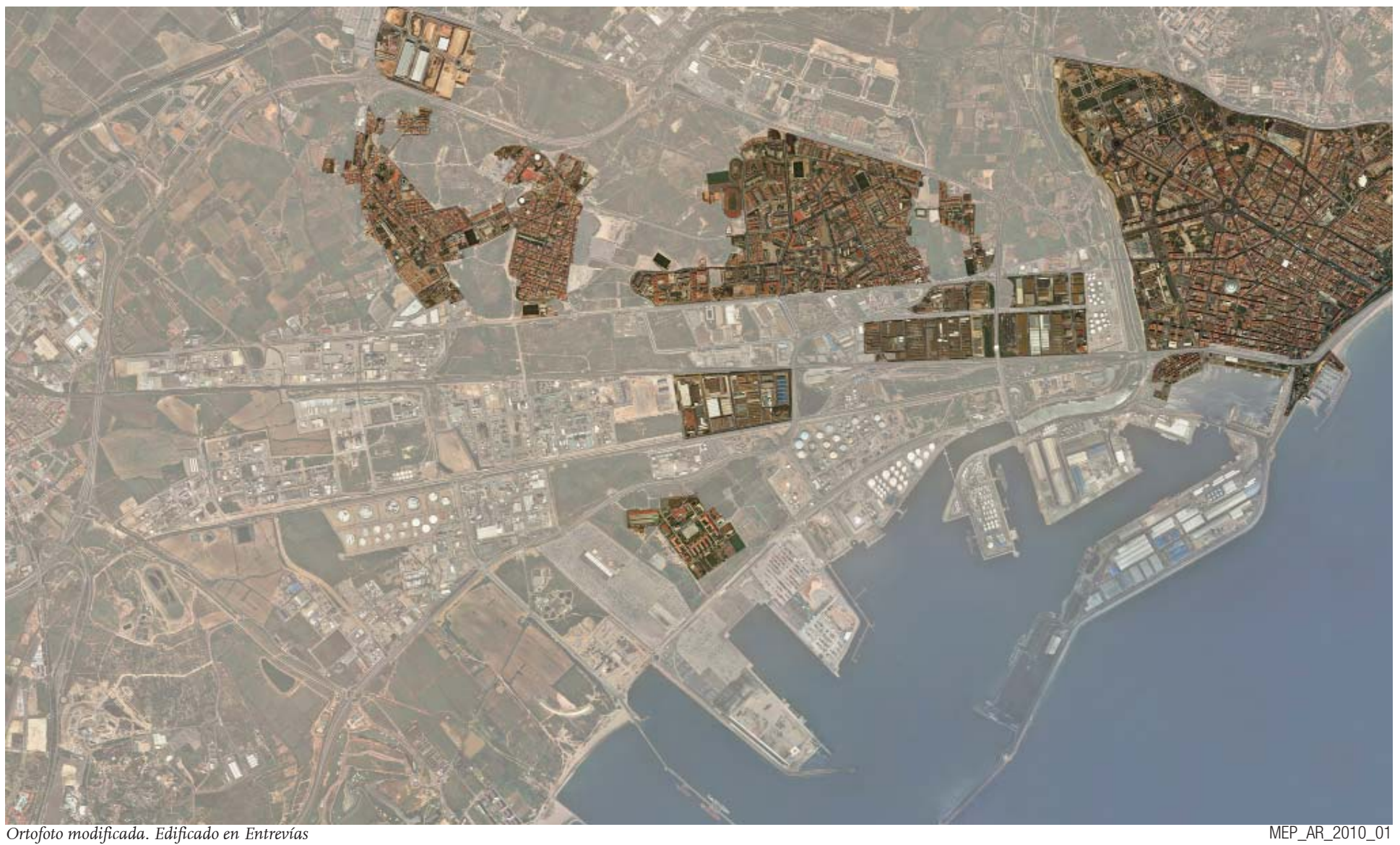



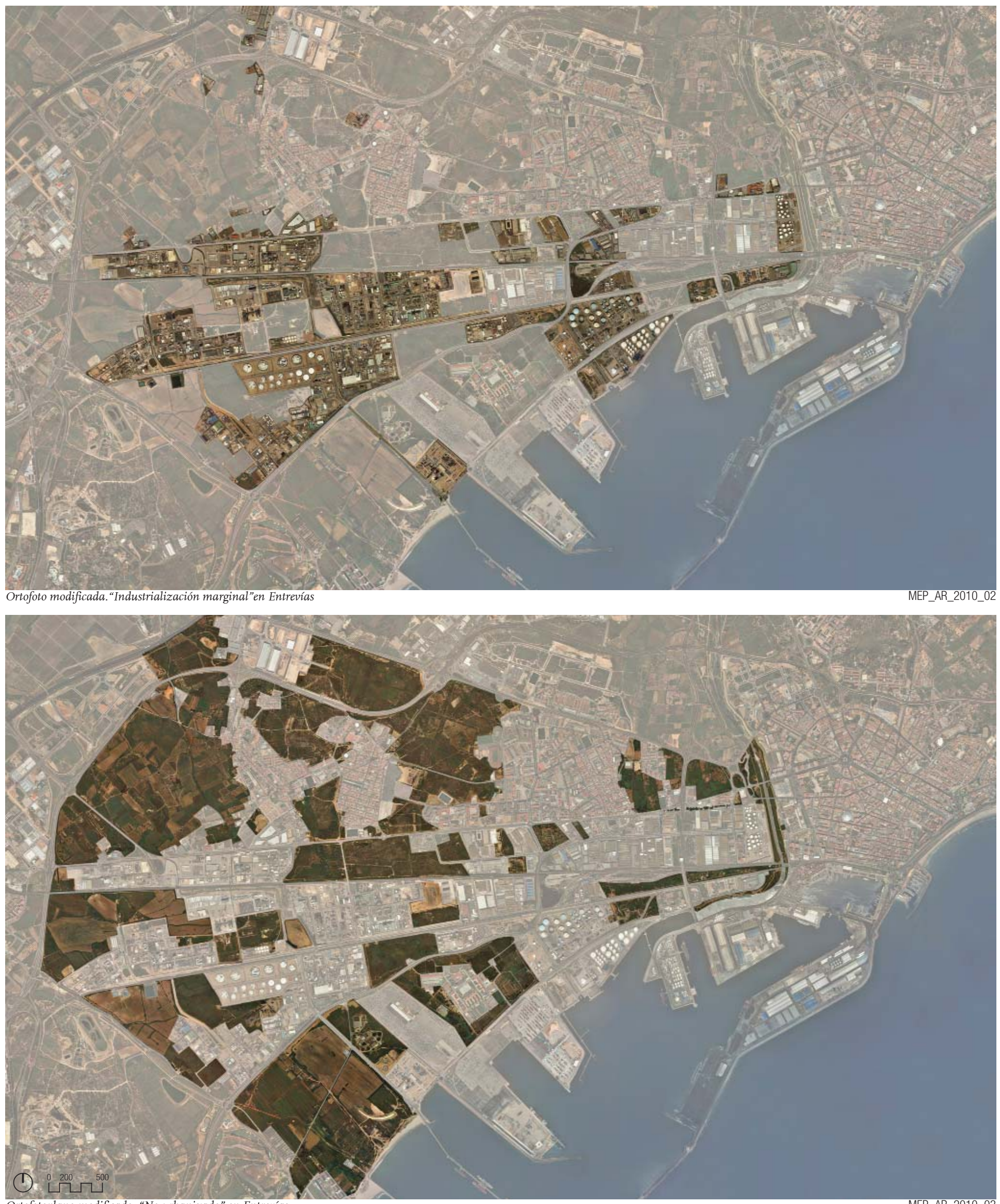


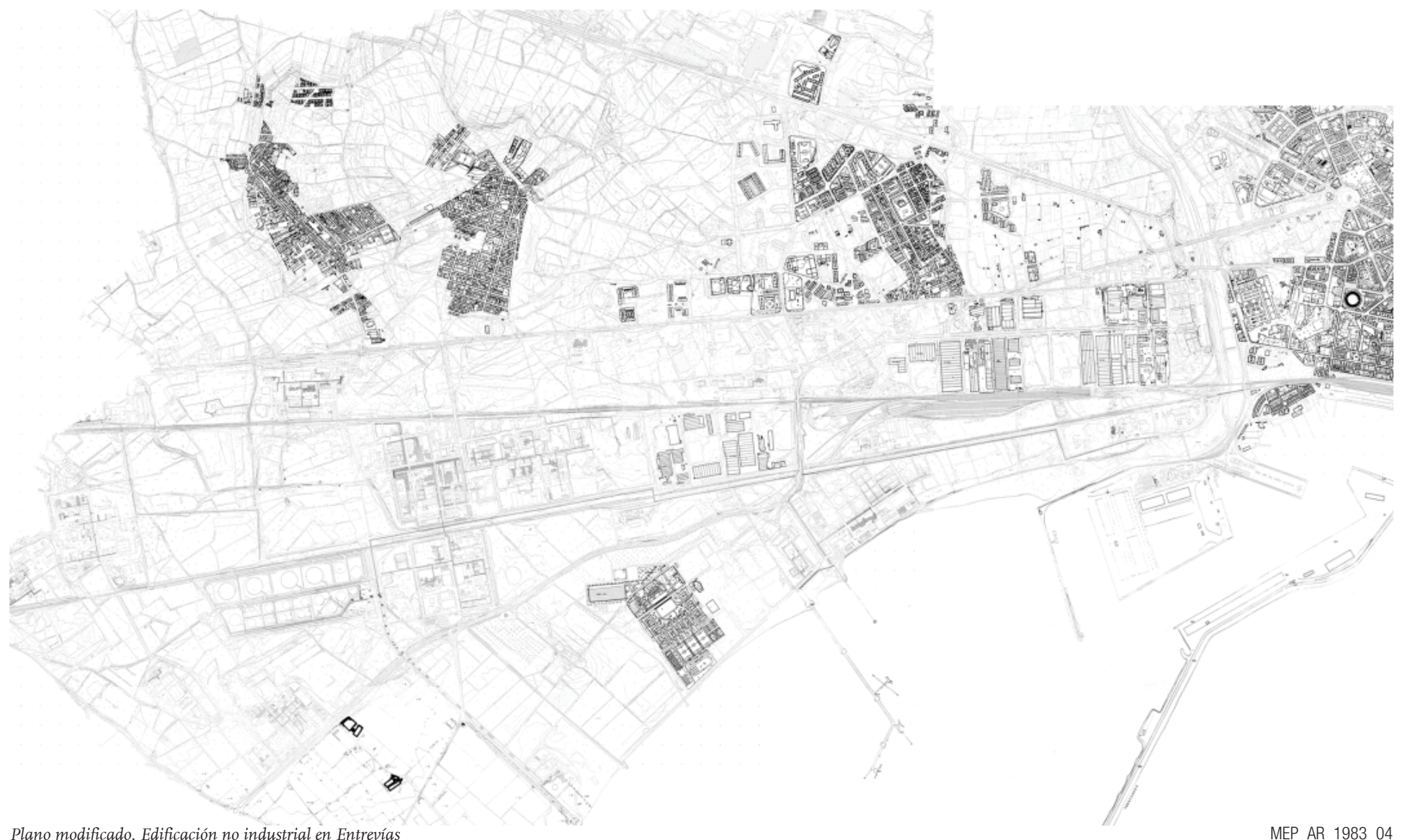

Plano modificado. Edificación no industrial en Entrevías

MEP_AR_1983_04

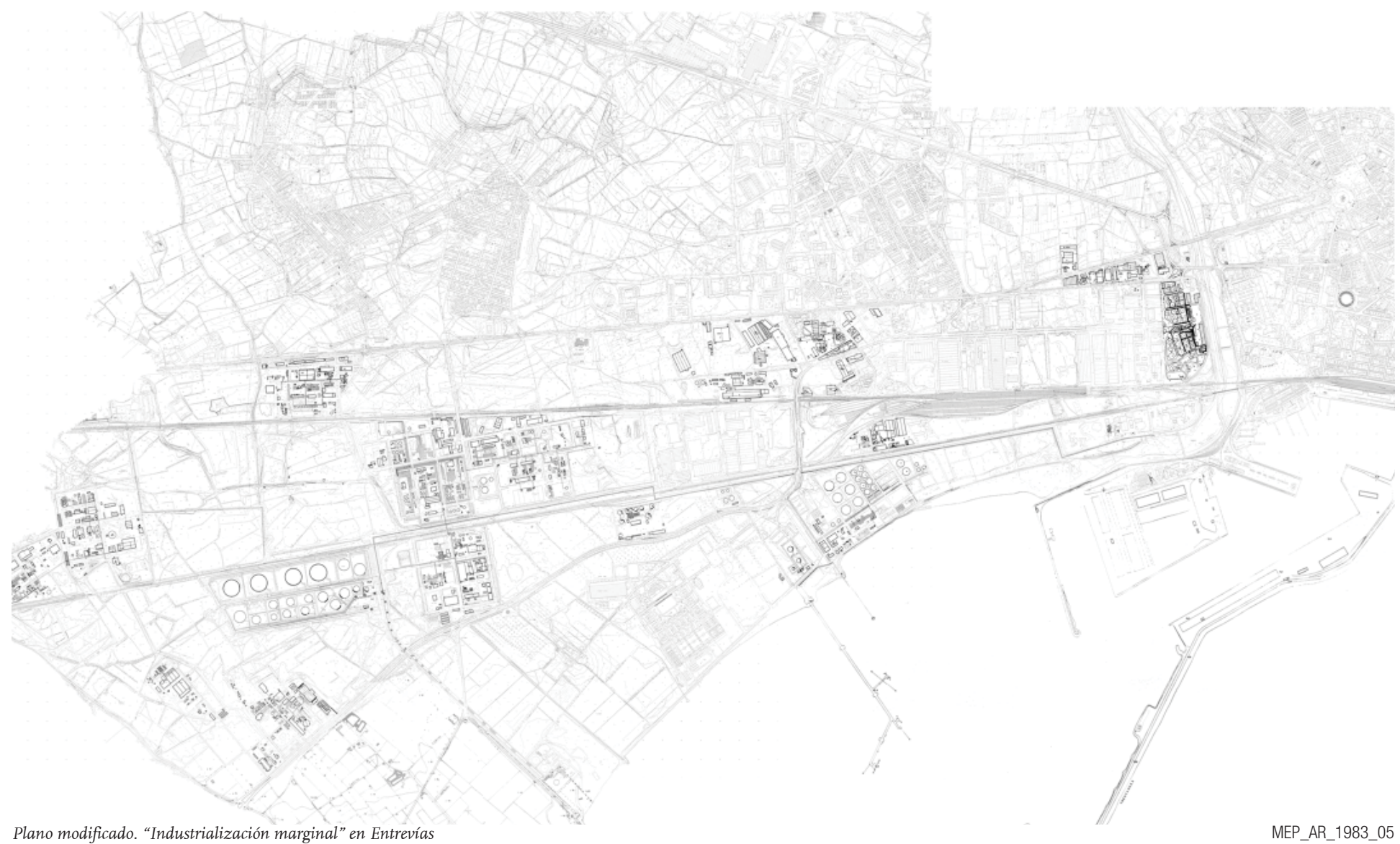




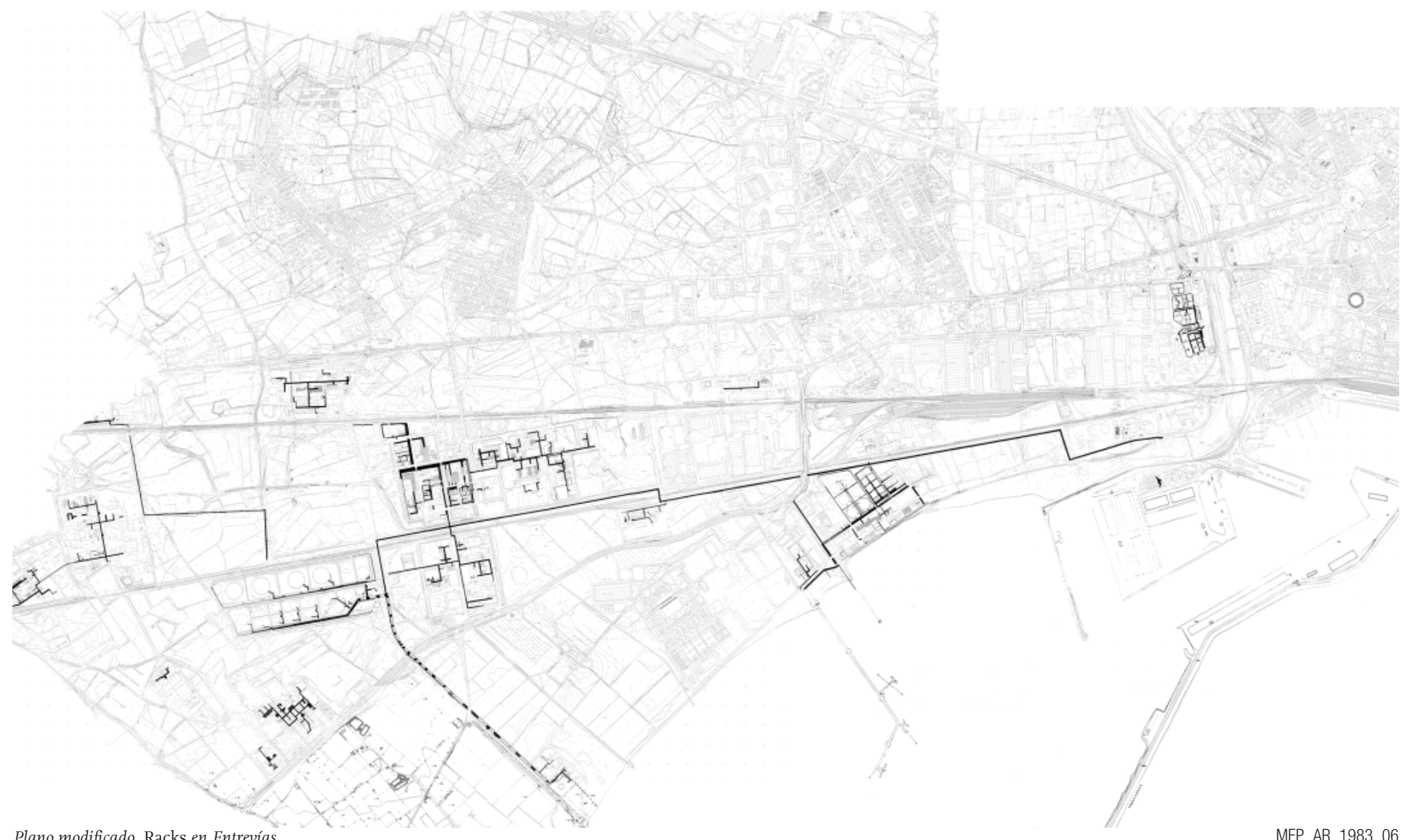

Plano modificado. Racks en Entrevías
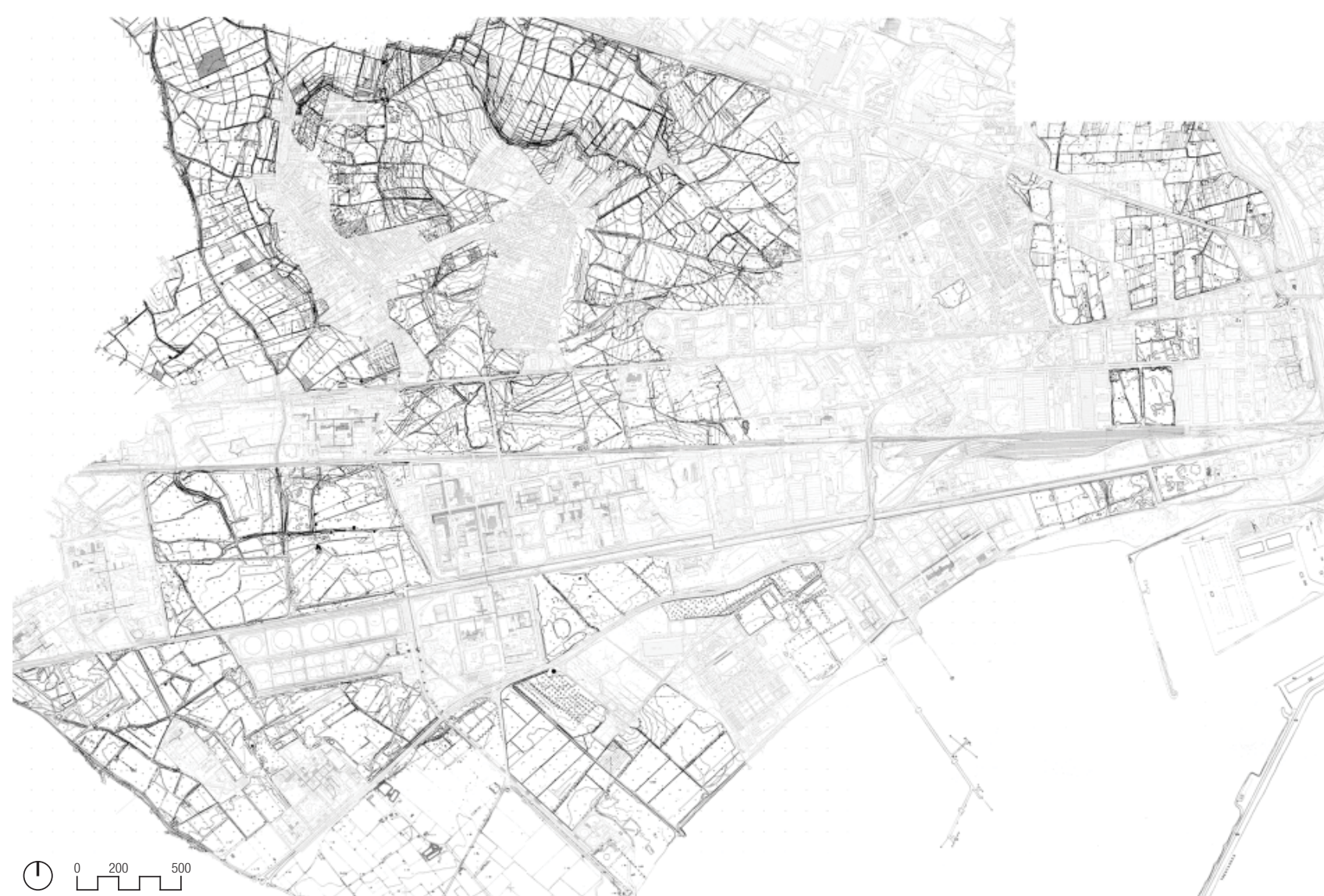

Plano modificado. “No urbanizado" en Entrevías
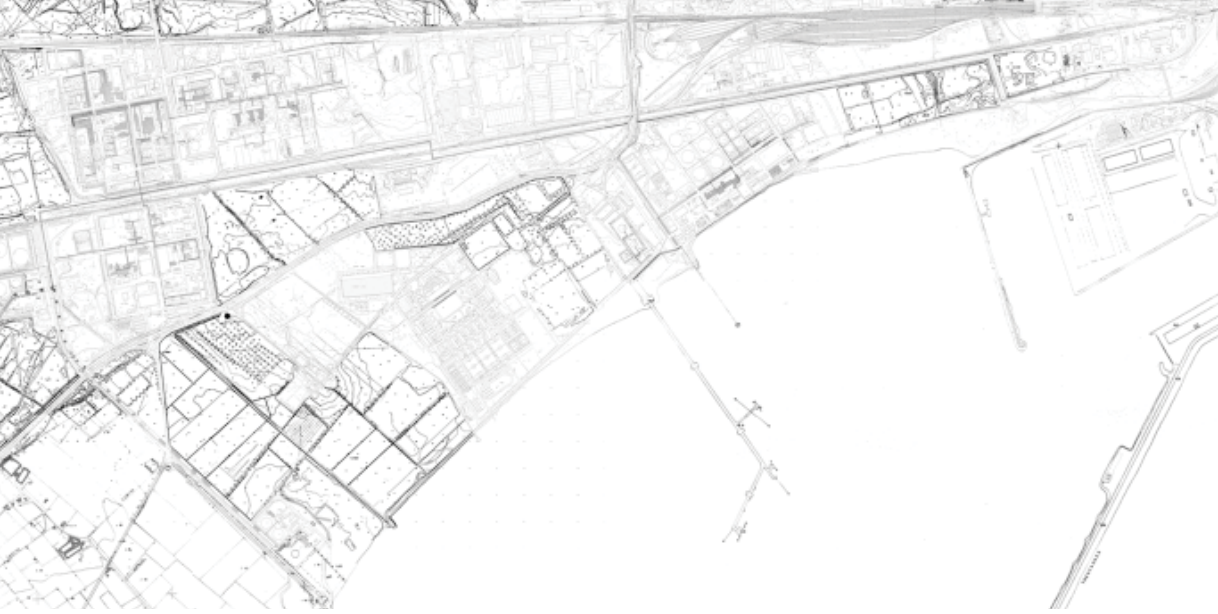

MEP_AR_1983_07 

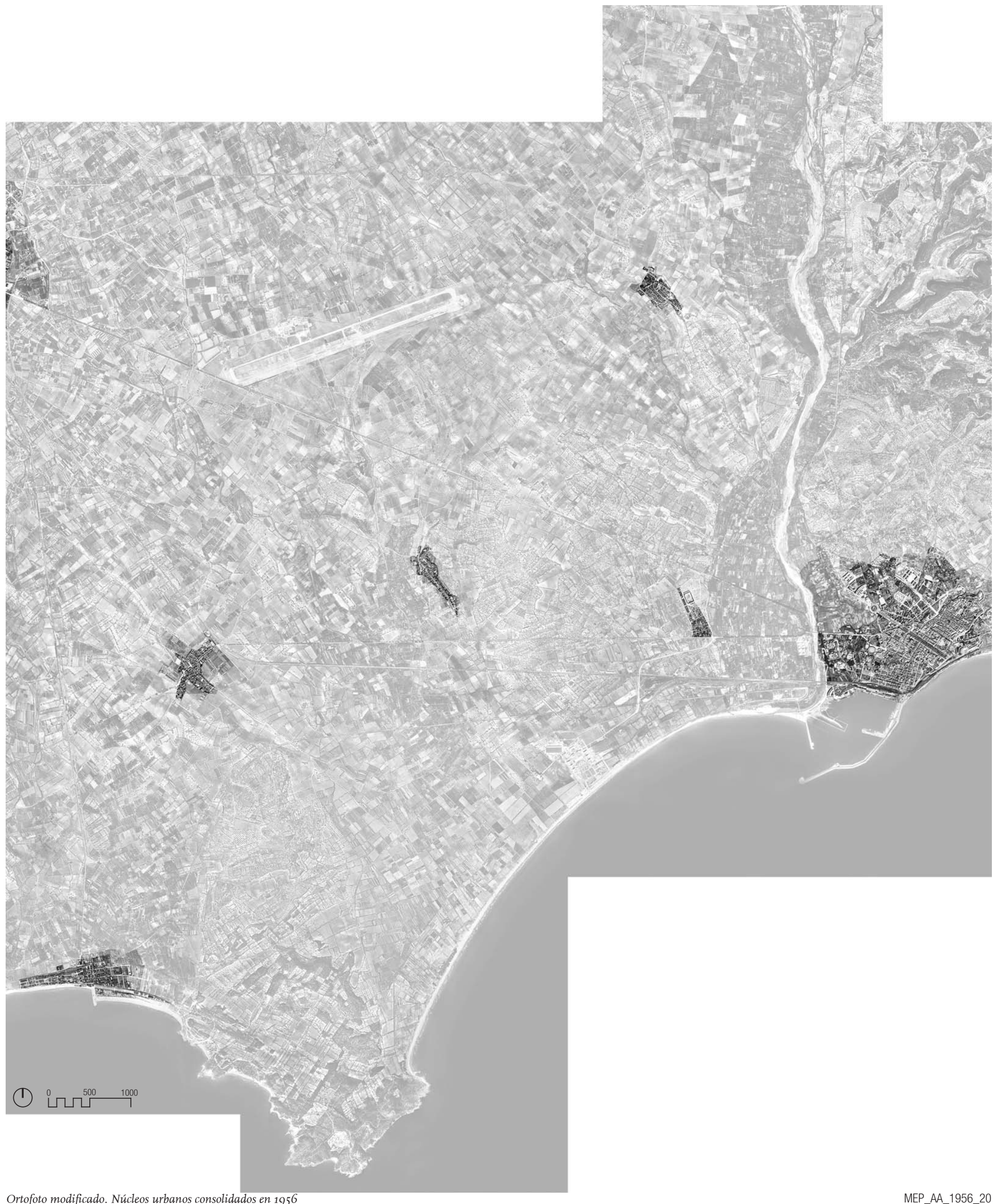


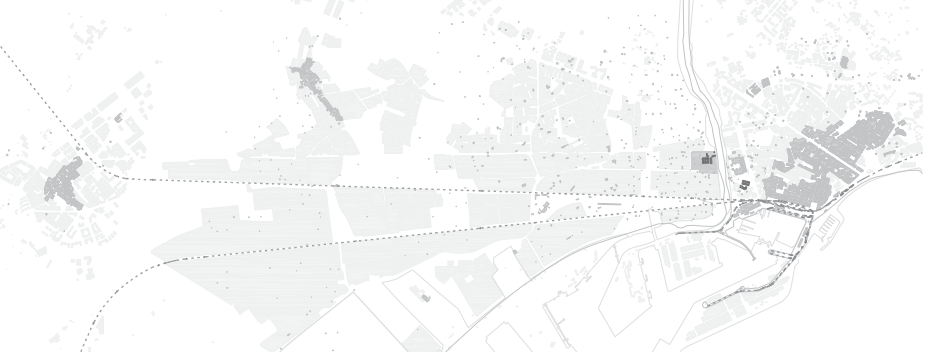

_AM_1930_01

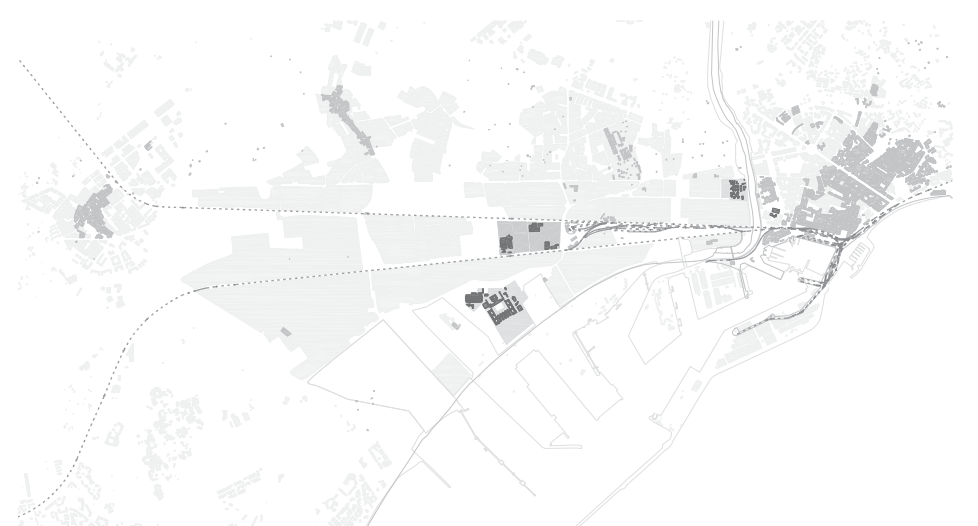

MEP_AM_1956_03

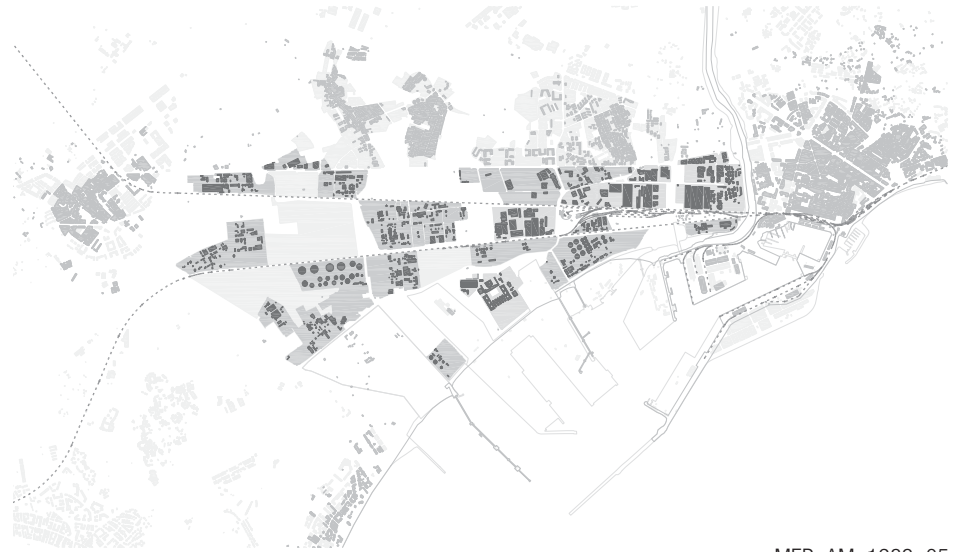

MEP_AM_1983_05

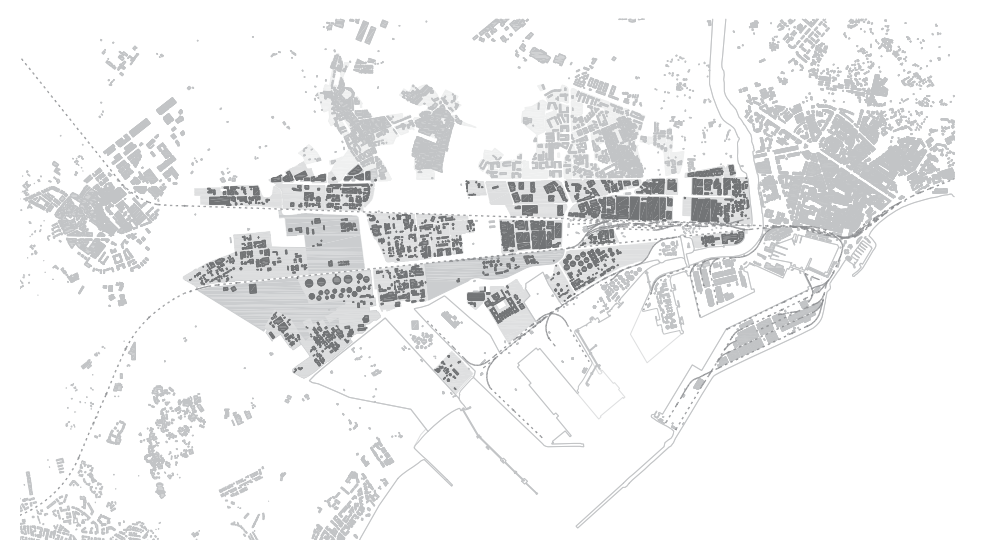

MEP_AM_2009_07

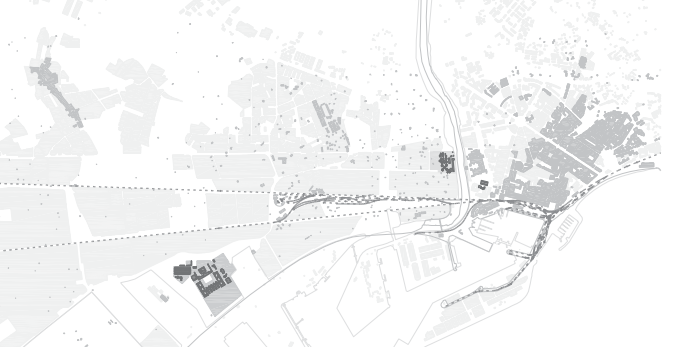

MEP_AM_1952_02

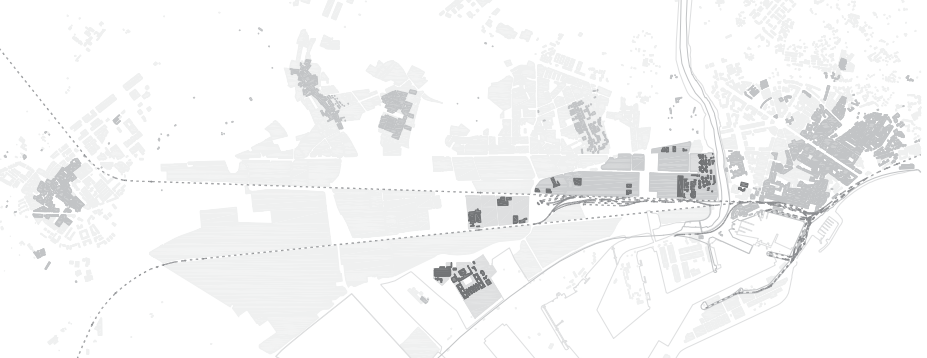

MEP_AM_1964_04

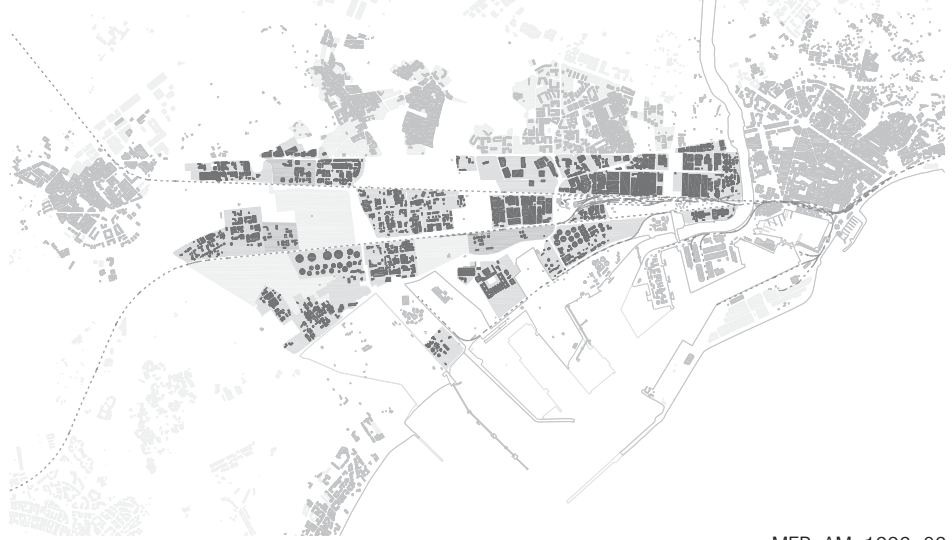

MEP_AM_1996_06

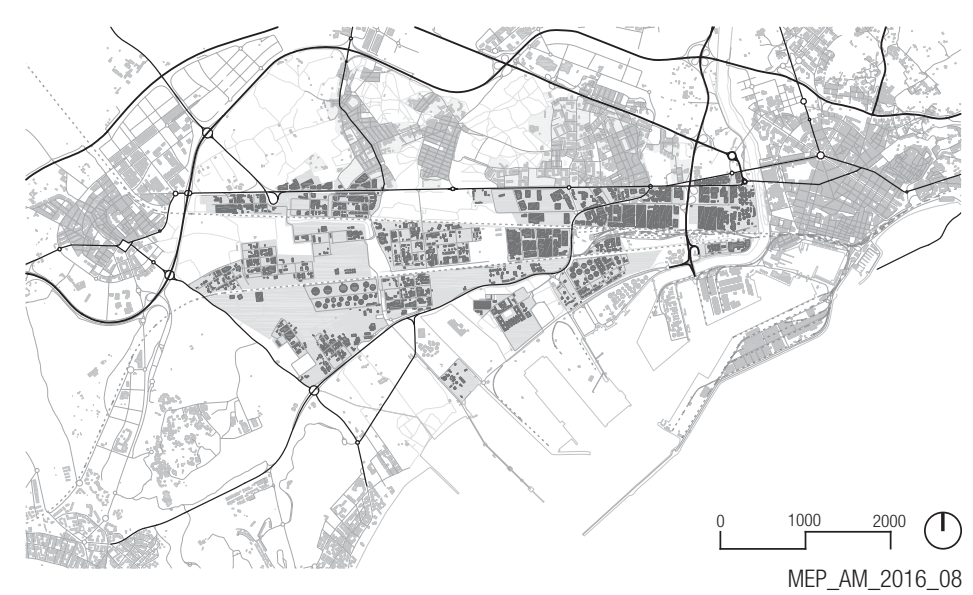


Secuencia de 1939. Topografía

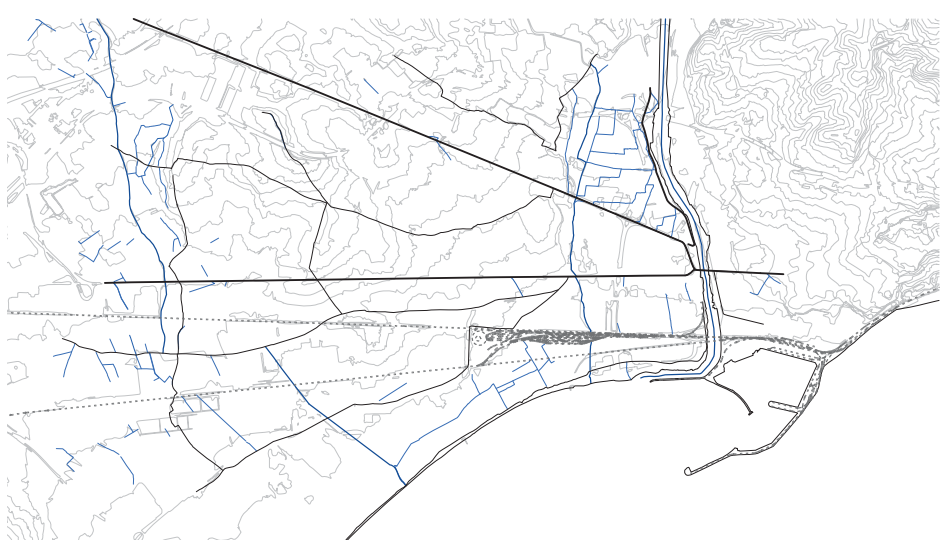

Secuencia de 1939. Red de caminos principales

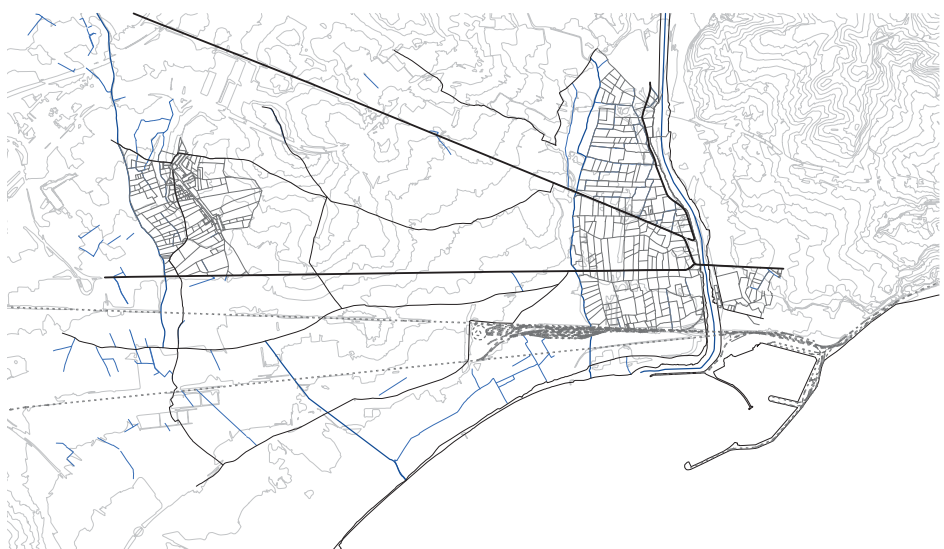

Secuencia de 1939. Parcelación rústica de regadío

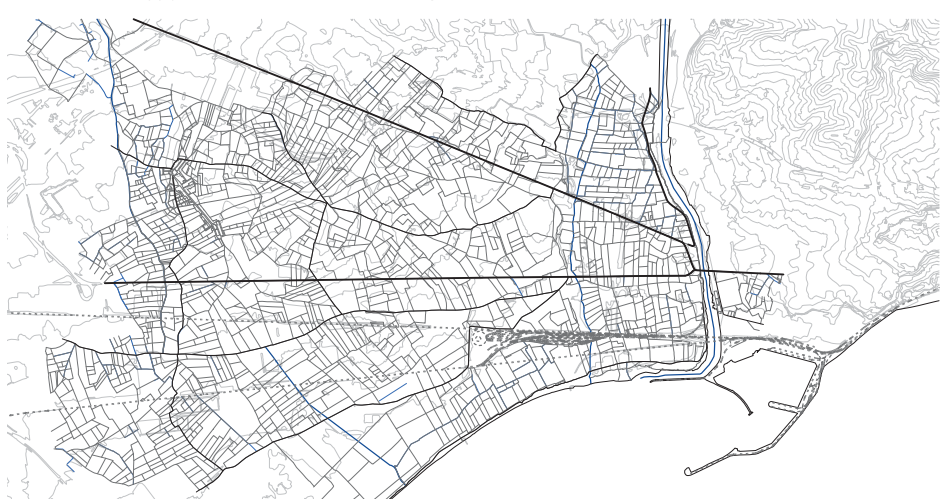

Secuencia de 1939. Parcelación rústica de costa

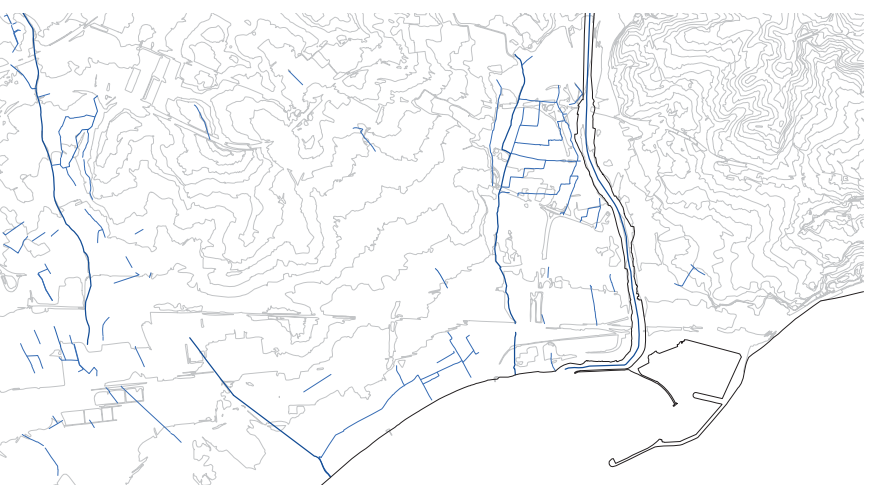

MEP_AP_1932_02

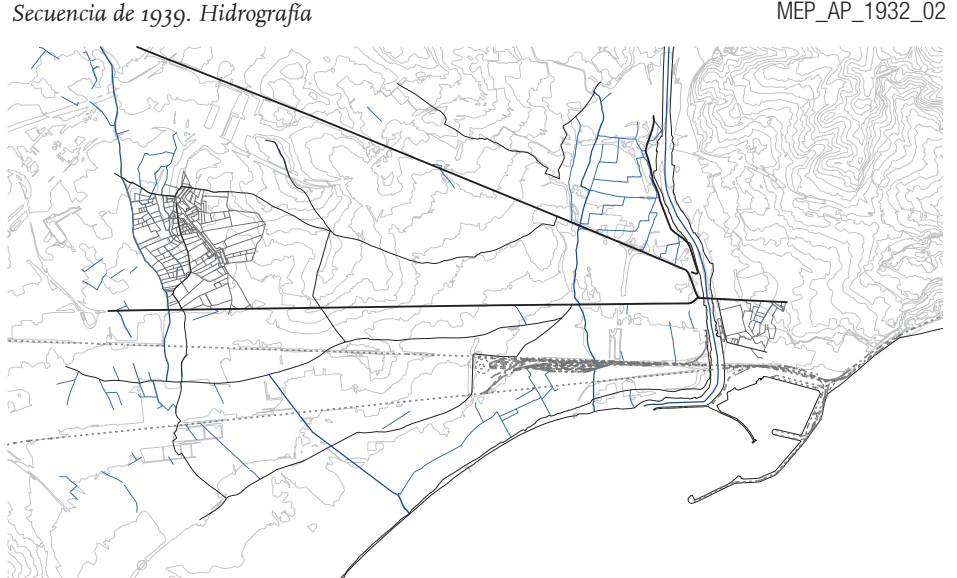

Secuencia de 1939. Parcelación urbana

MEP_AP_1932_04
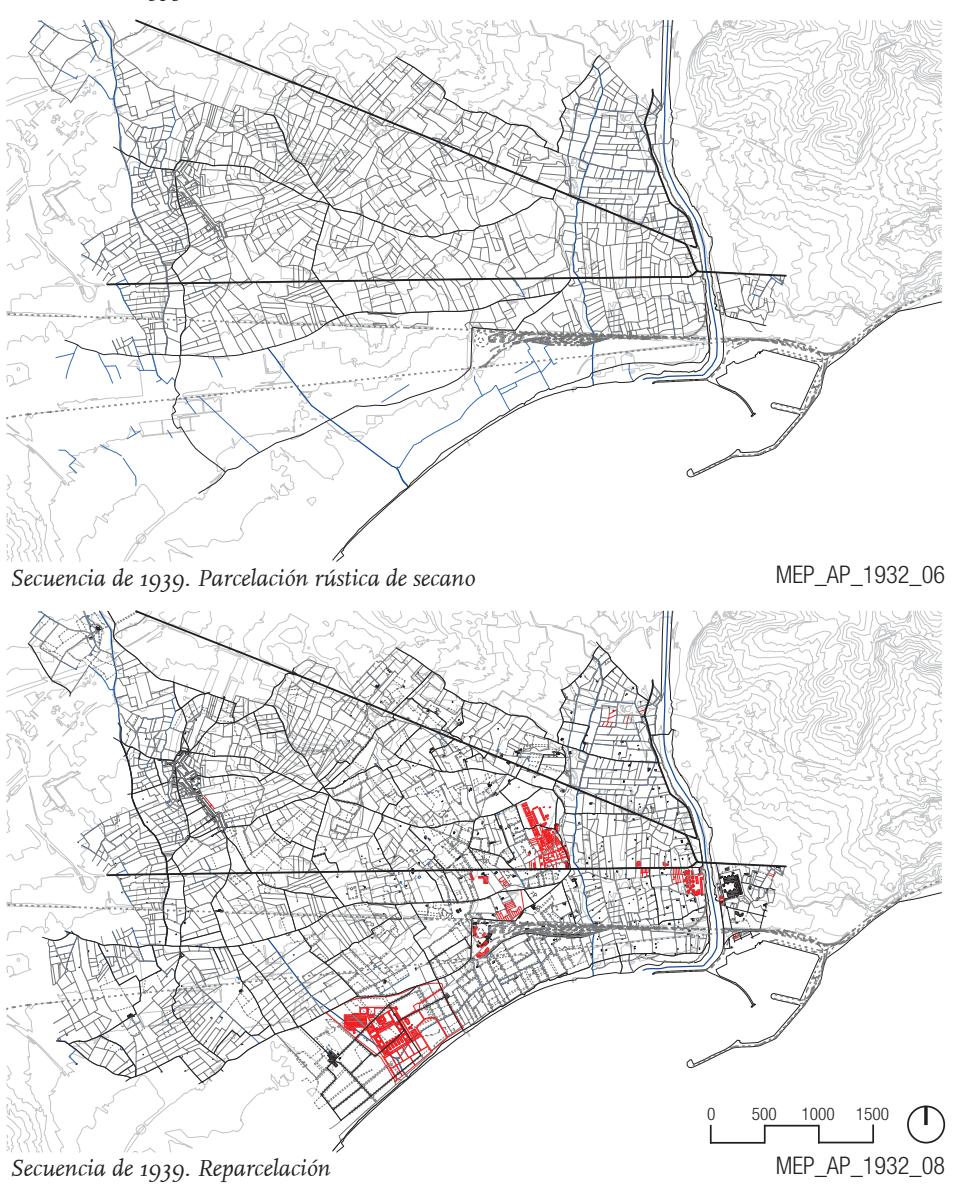


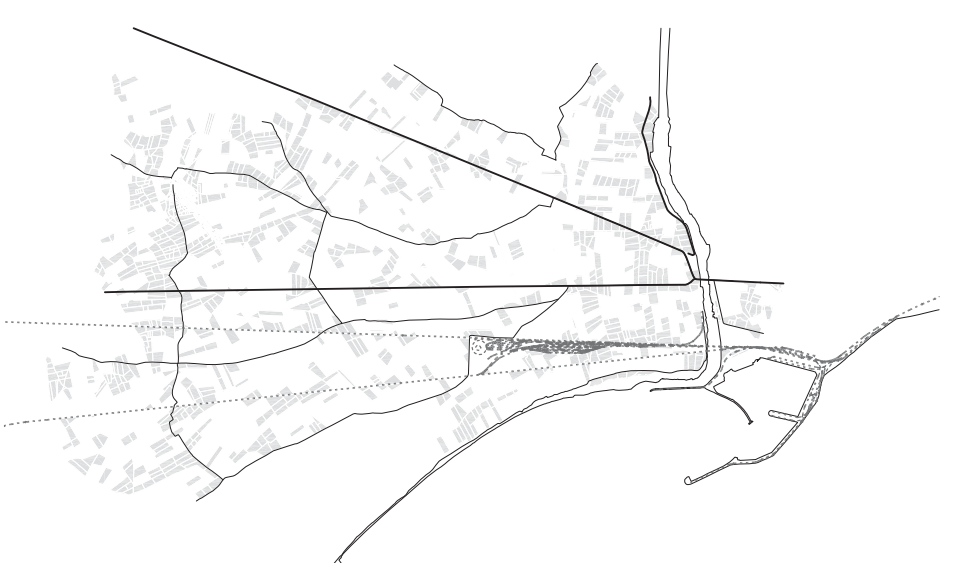

Parcelas con superficie inferior a $10.000 \mathrm{~m}^{2}$

MEP_AP_1932_09
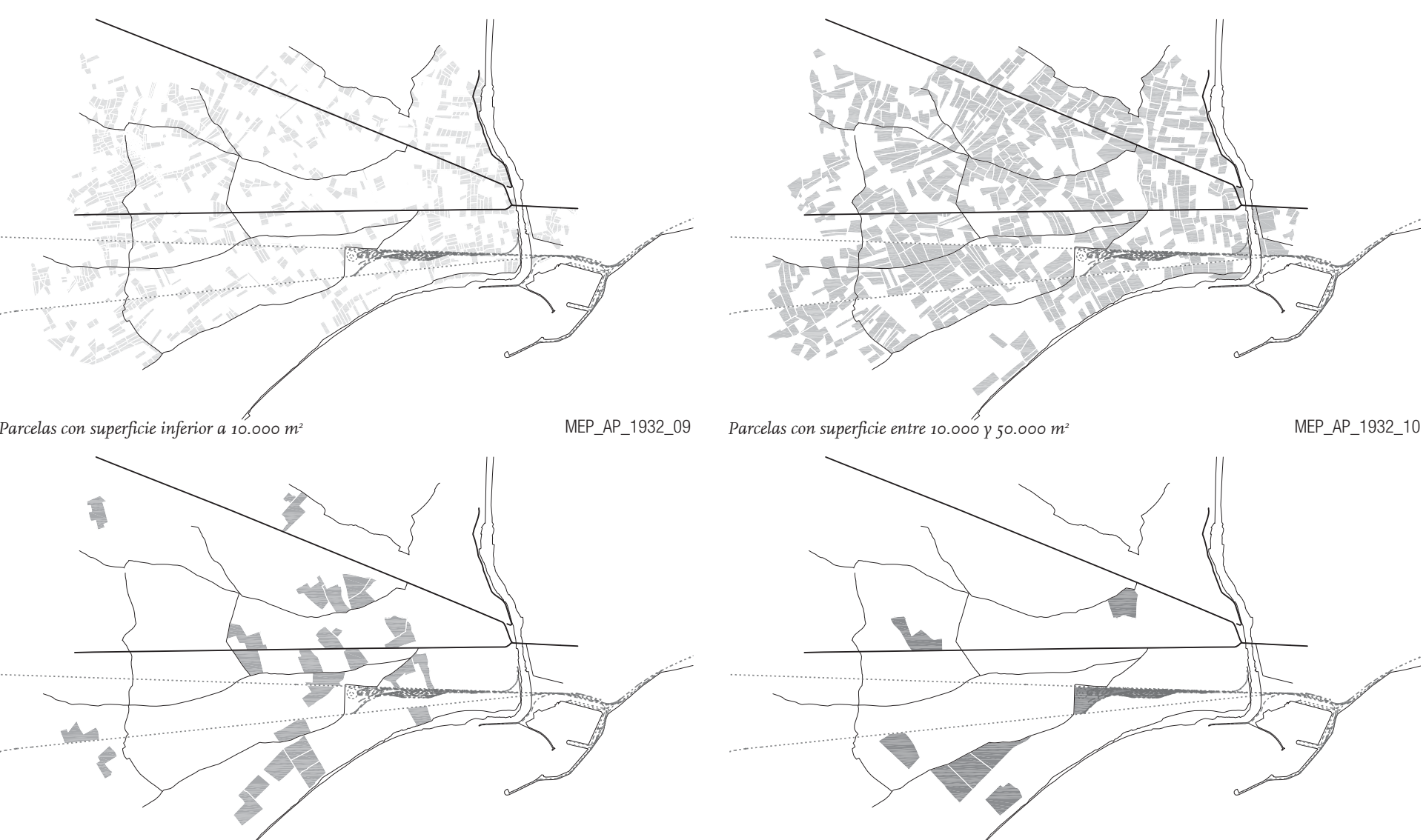

Parcelas con superficie entre 50.000 Y $100.000 \mathrm{~m}^{2}$

MEP_AP_1932_11

Parcelas con superficie entre 10.000 Y $50.000 \mathrm{~m}^{2}$

MEP_AP_1932_10
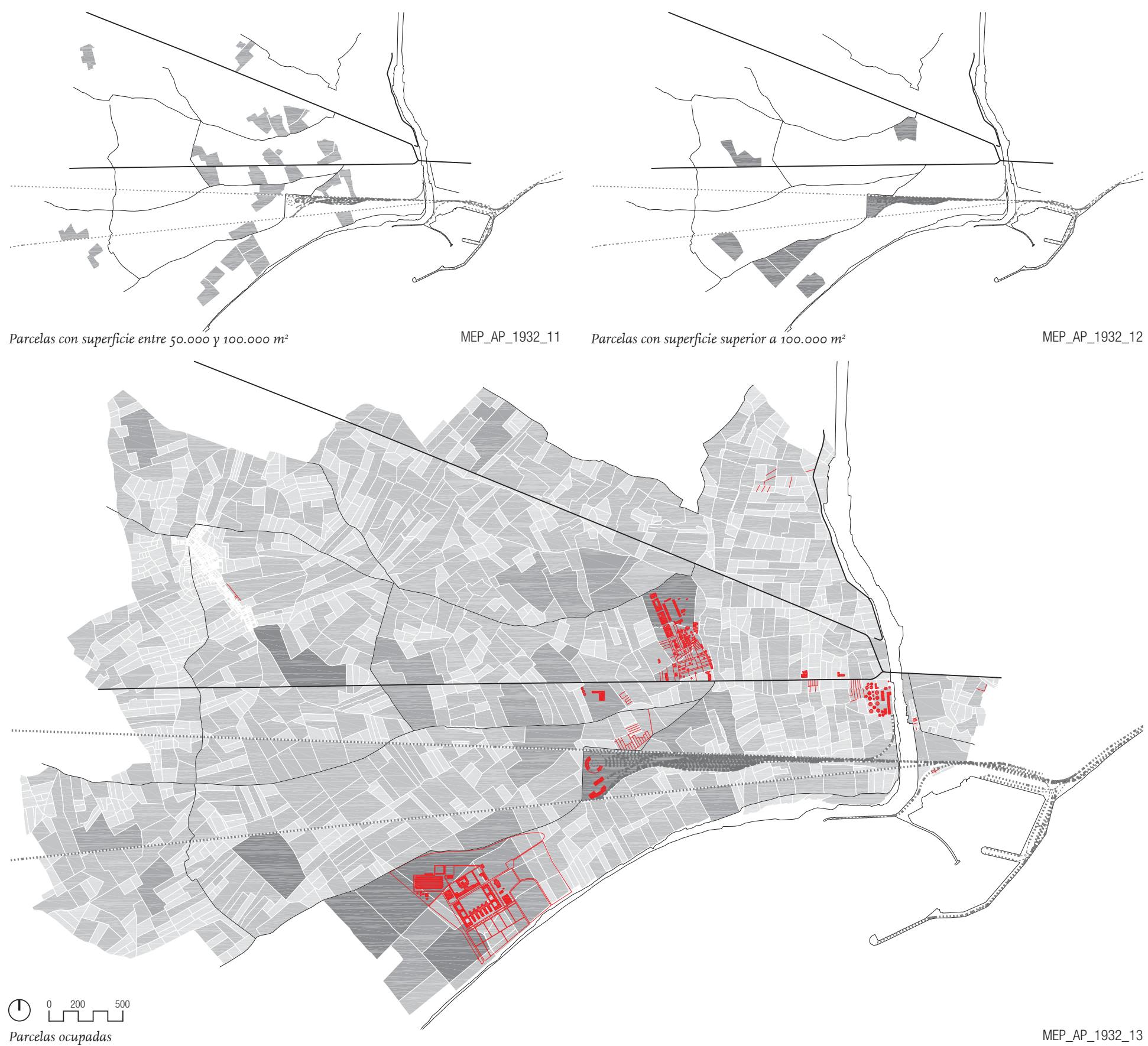

Parcelas con superficie superior a $100.000 \mathrm{~m}^{2}$ 

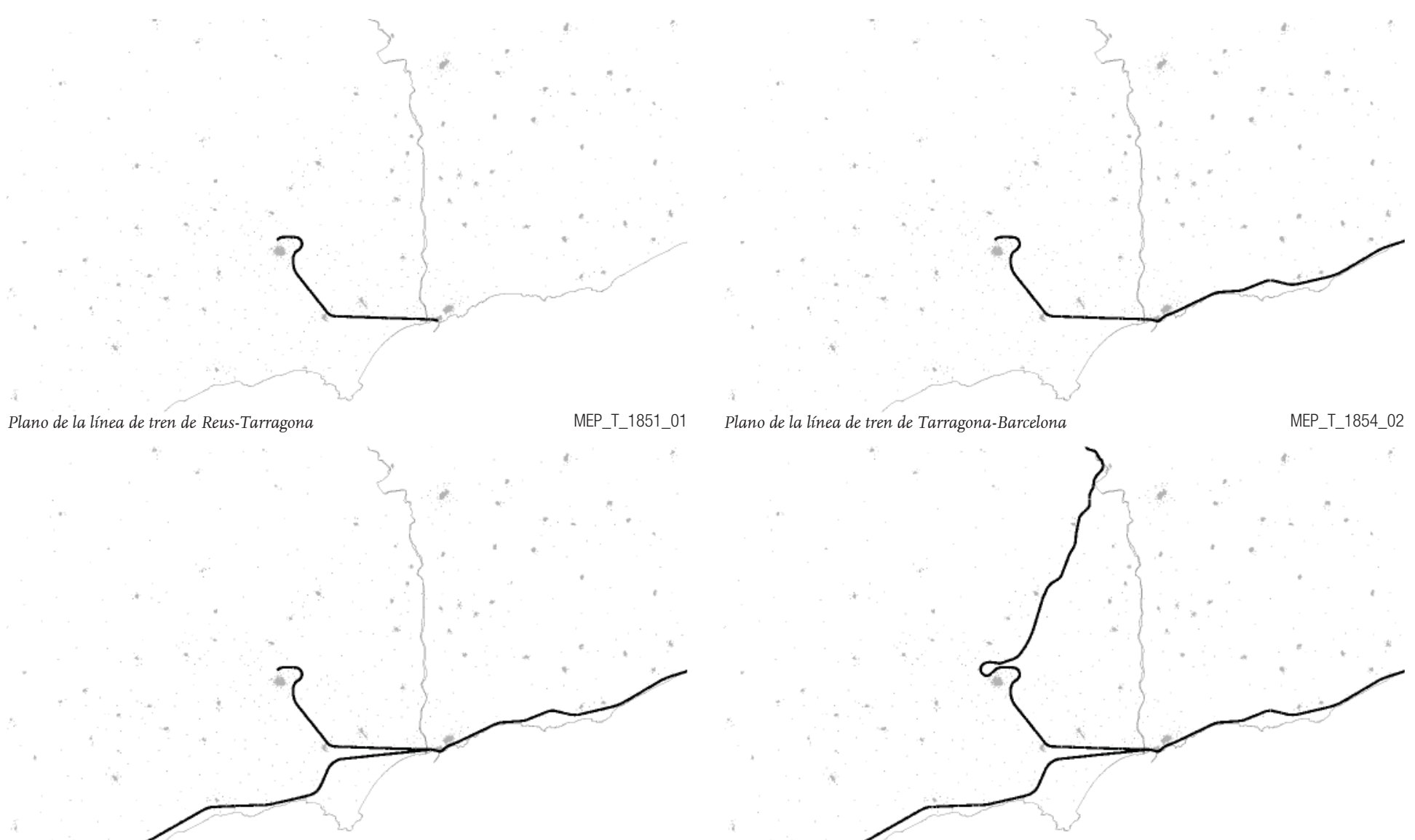

Plano de la línea de tren de Valencia-Tarragona

MEP_T_1855_03
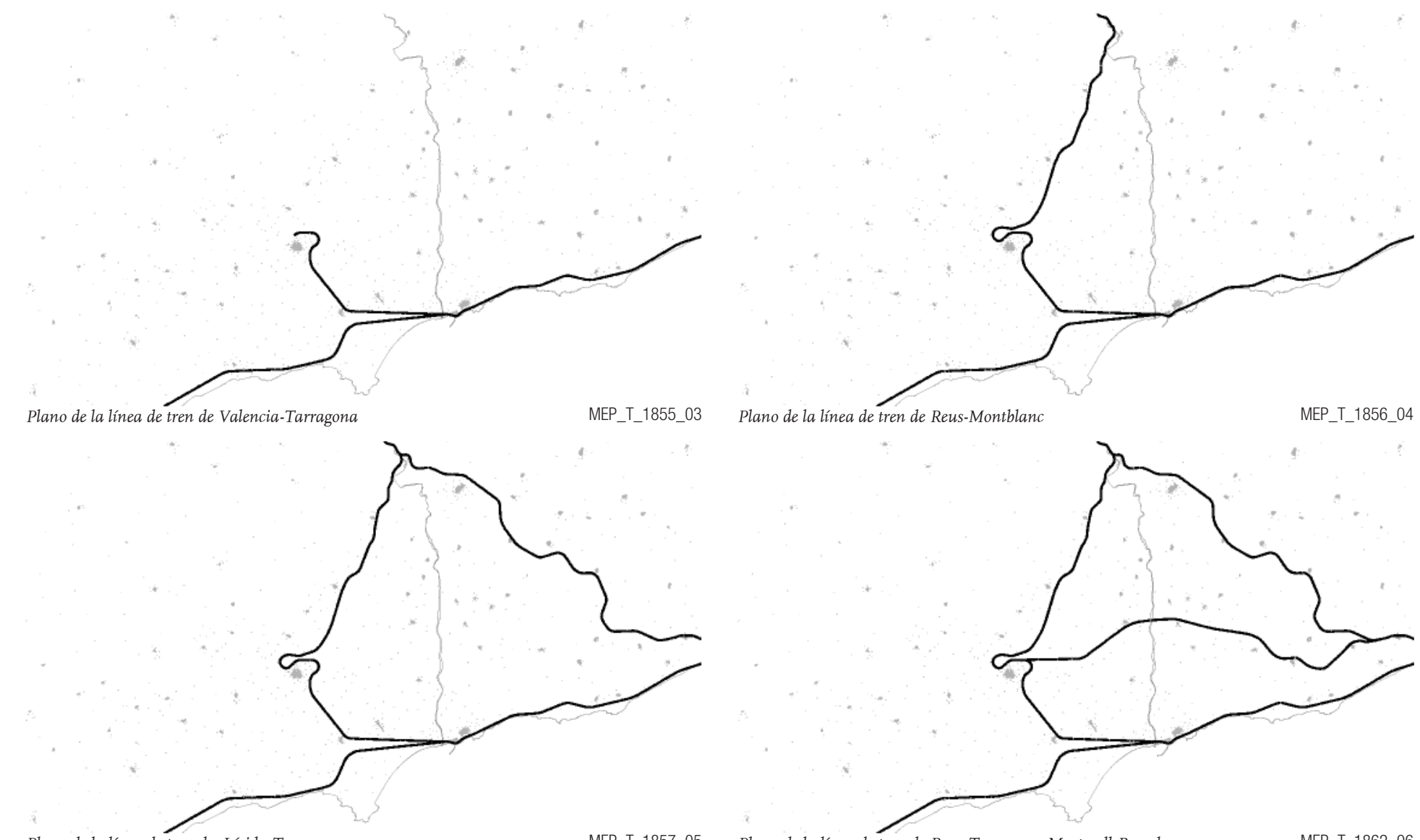

Plano de la línea de tren de Reus-Montblanc

MEP_T_1856_04

Plano de la línea de tren de Lérida-Tarragona

MEP_T_1857_05

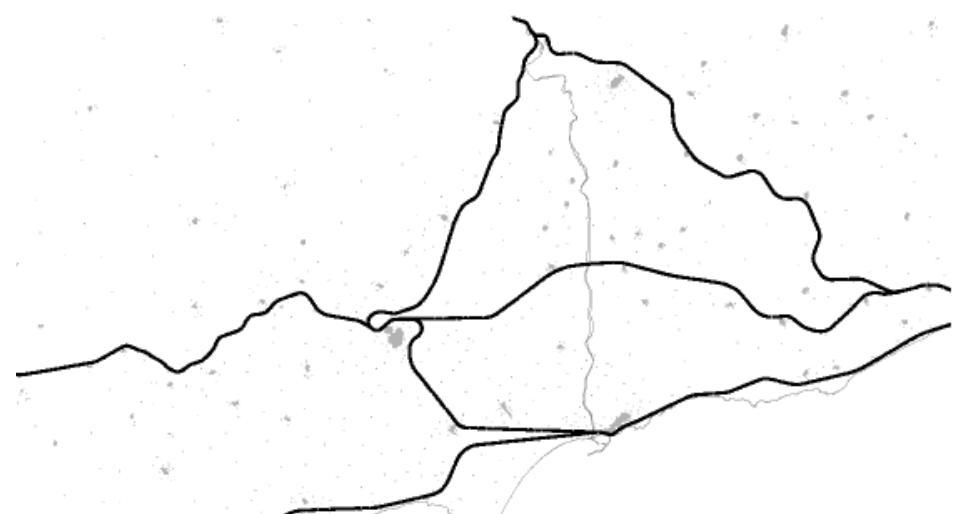

Plano de la línea de tren de Reus - Roda de Bará

MEP_T_1884_07

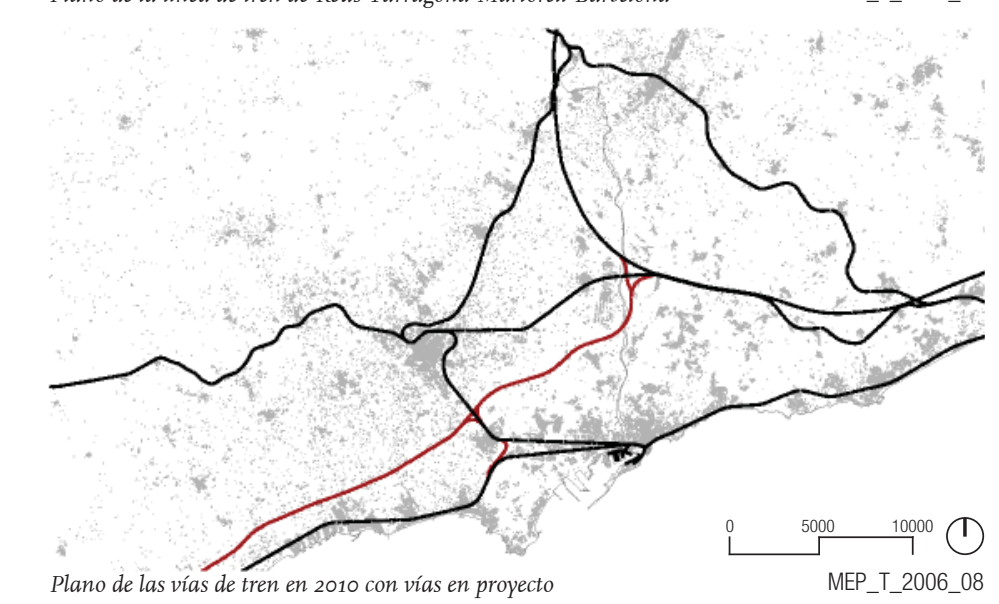




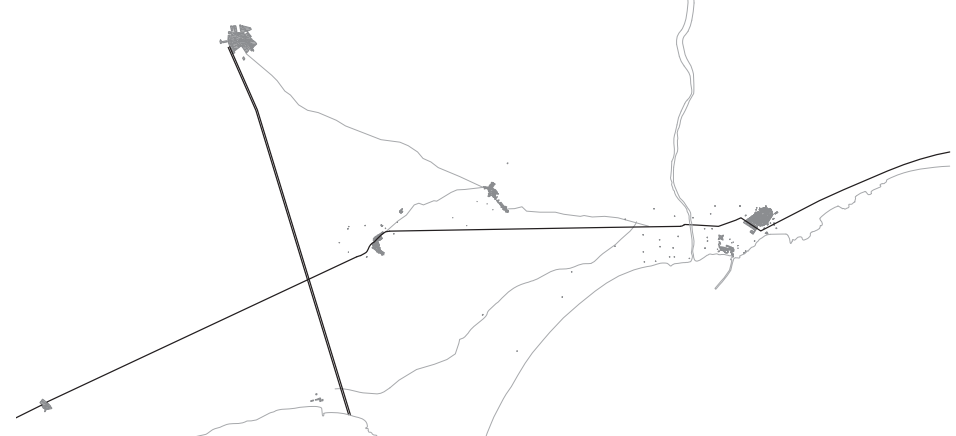

Secuencia viaria. Proyecto del canal de Reus

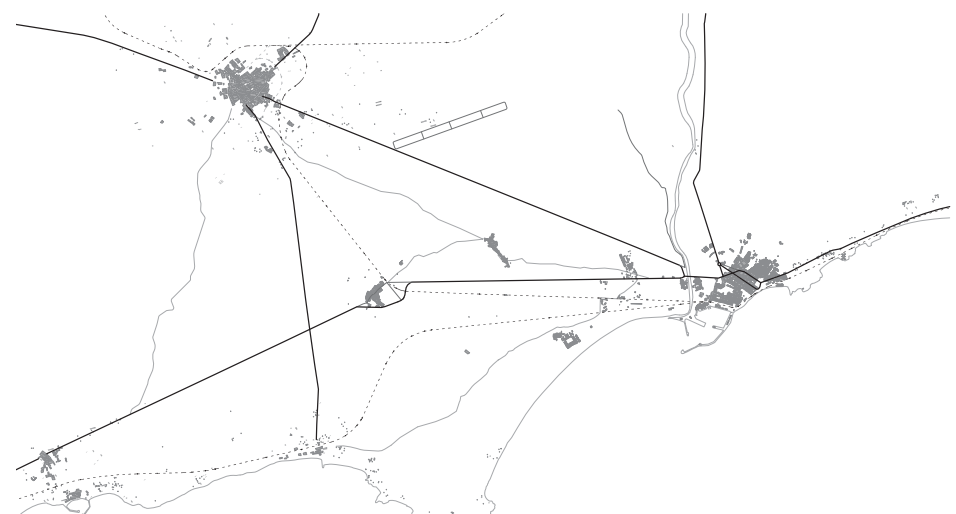

Secuencia viaria. Estado en 1956

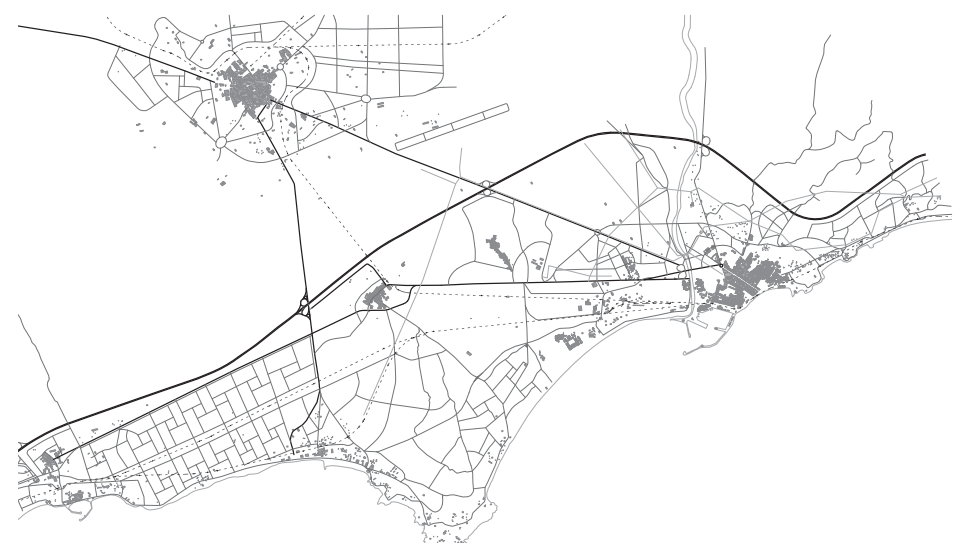

Secuencia viaria. Plan Especial de Ordenación de la Costa de Tarragona

MEP V 196305

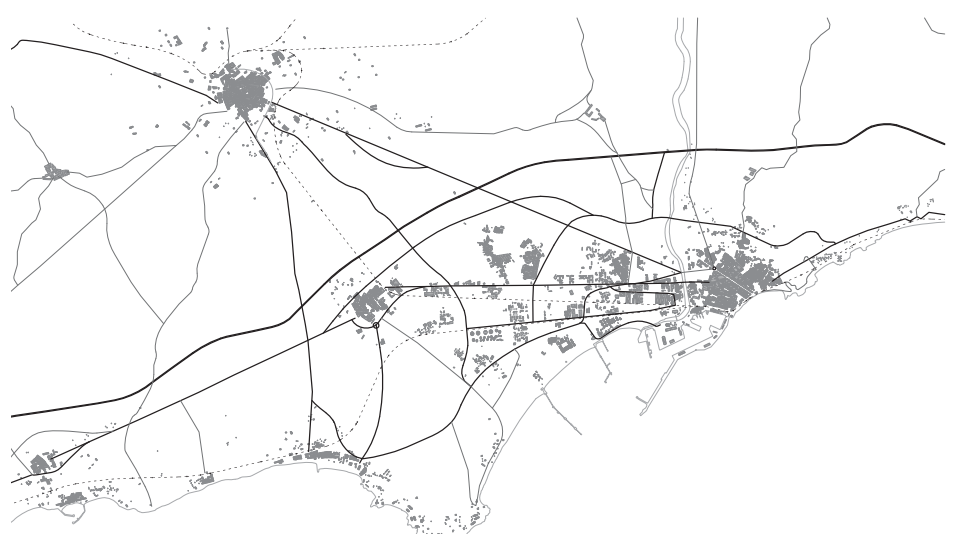

Secuencia viaria. Comisión de Municipios del Camp de 1980

MEP_V_1980_07

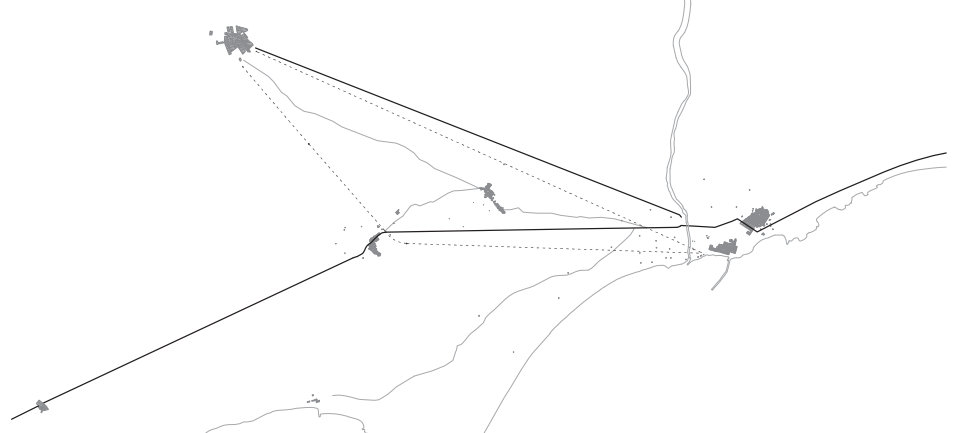

Secuencia viaria. Proyecto trazado Tarragona-Reus

MEP_V_1855_02

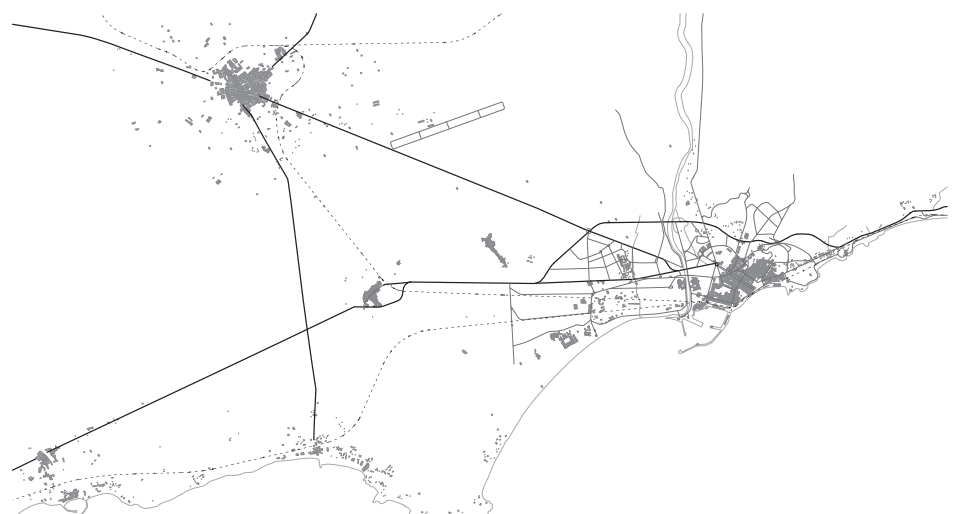

Secuencia viaria. Plan General de Ordenación de 1960

MEP_V_1960_04

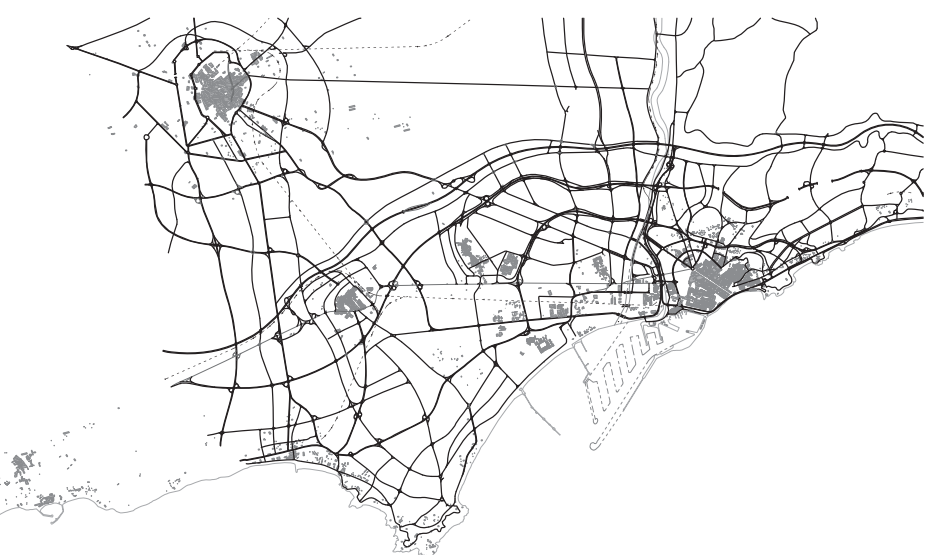

Secuencia viaria. Plan Comarcal de 1967

MEP V _ 1967_06

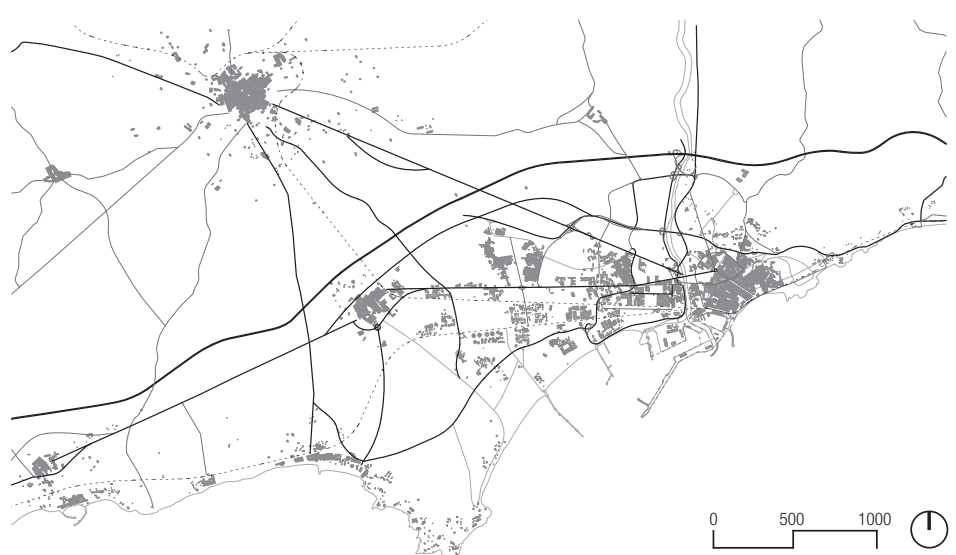

Secuencia viaria. Plan General de Ordenación Urbana de Tarragona de 1983 MEP_V_1983_08 


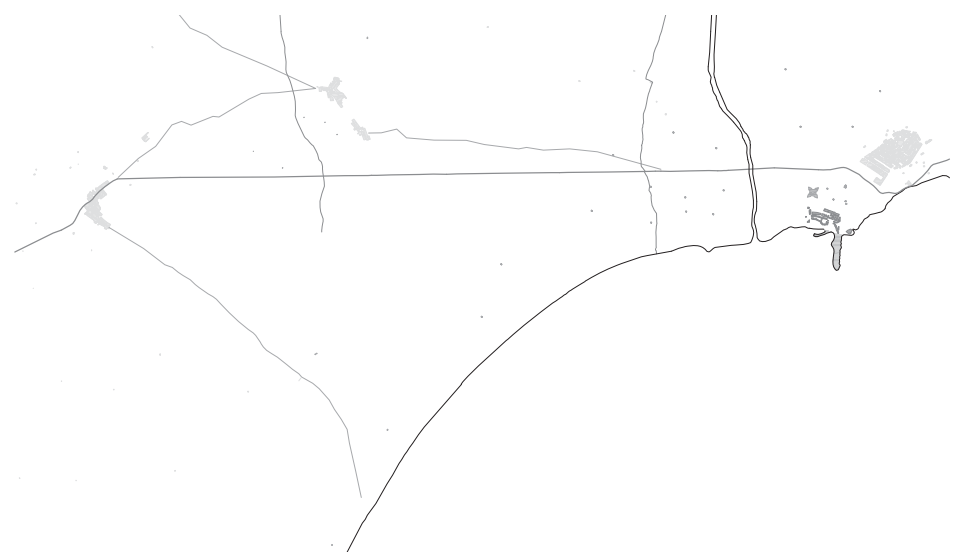

MEP_P_1801_01

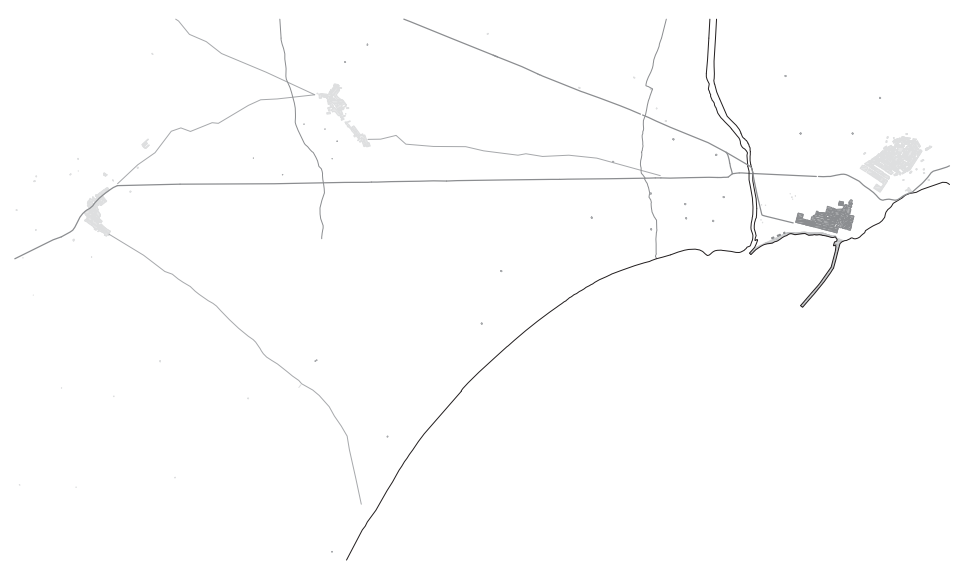

MEP_P_1855_03

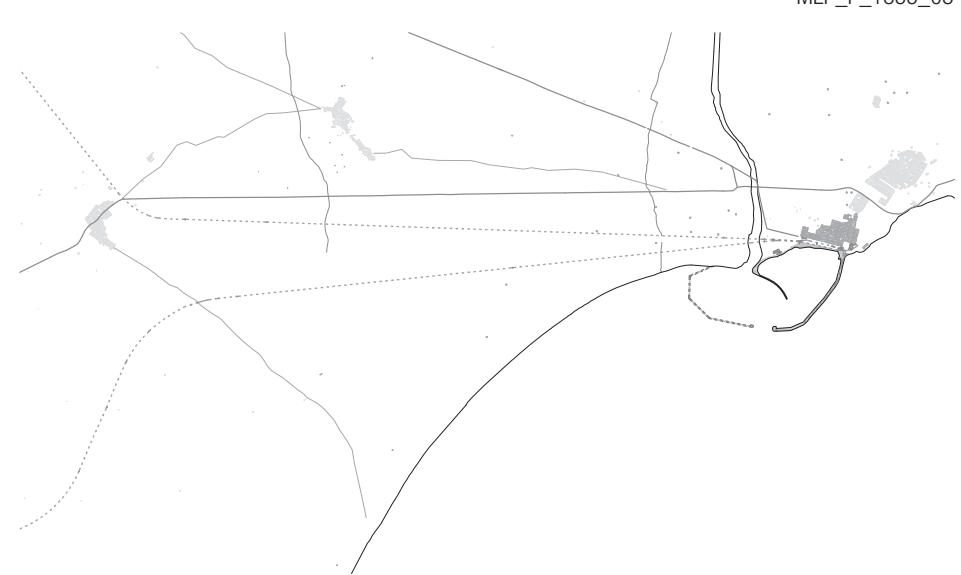

MEP_P_1876_05

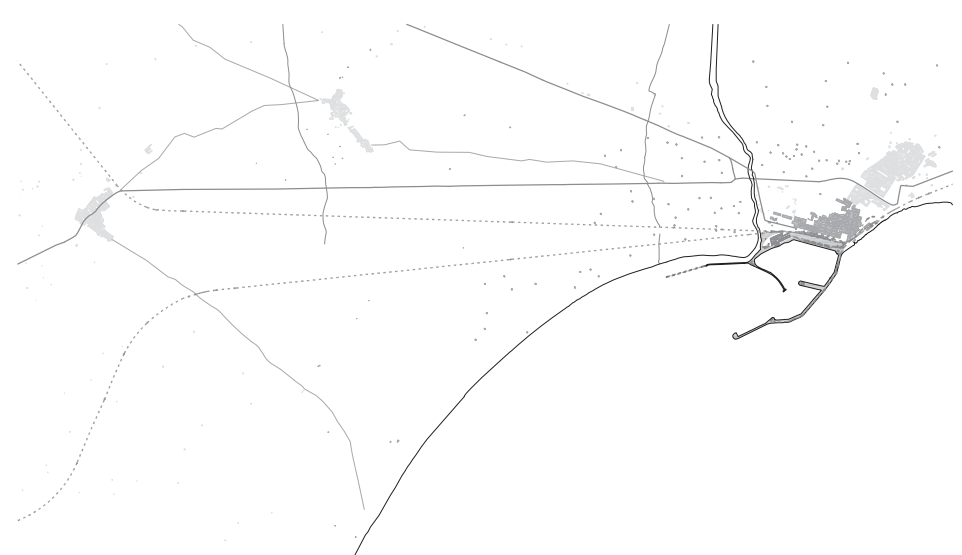

Secuencia cartográfica de crecimiento portuario

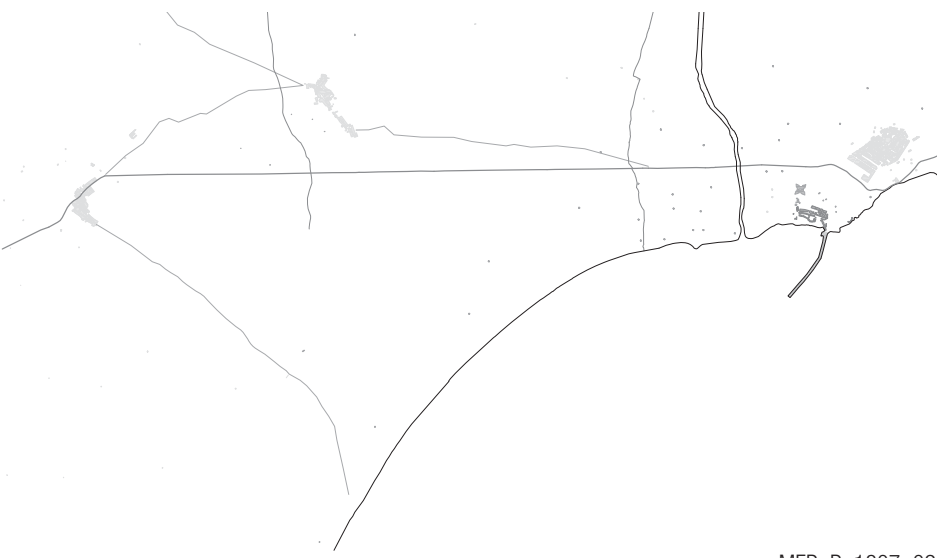

MEP_P_1807_02

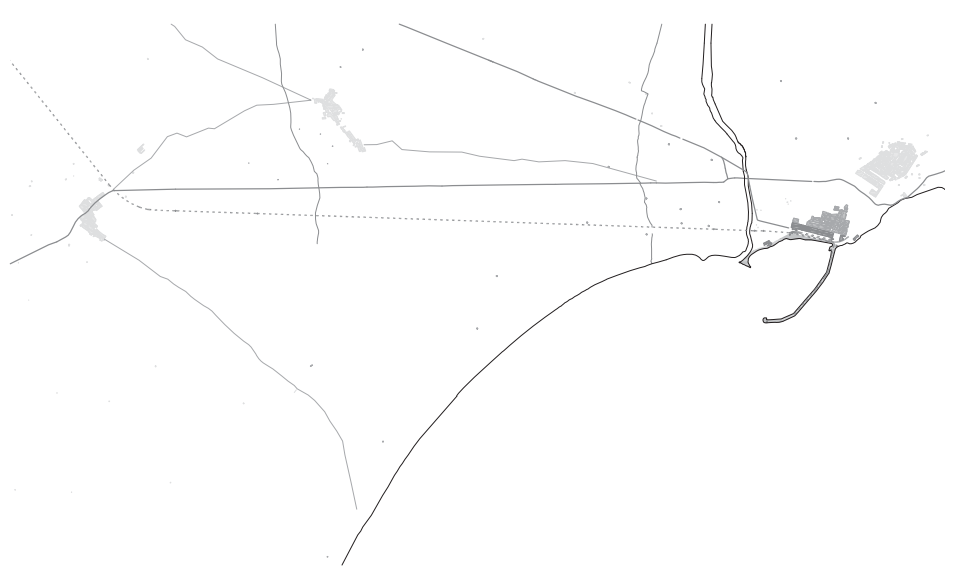

MEP_P_1860_04

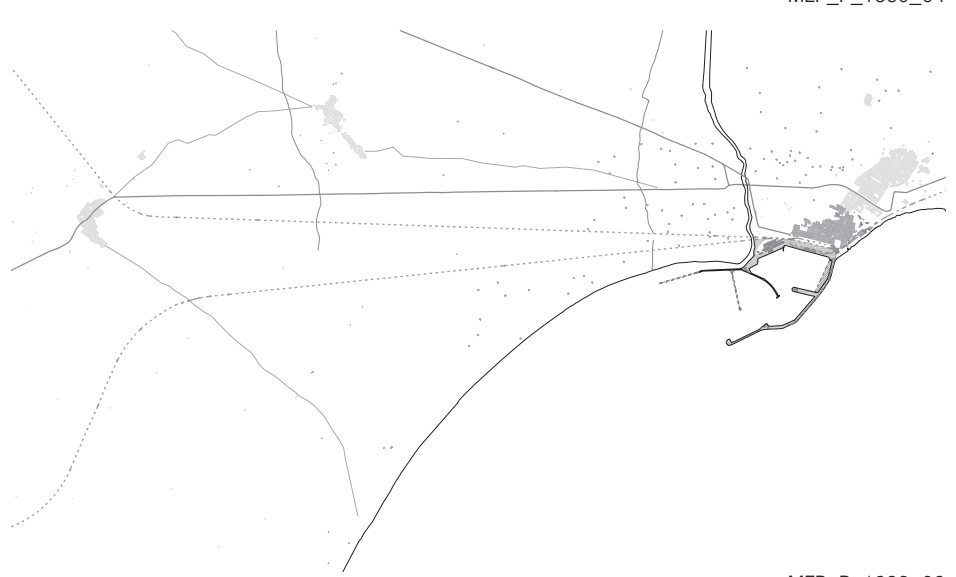

MEP_P_1882_06

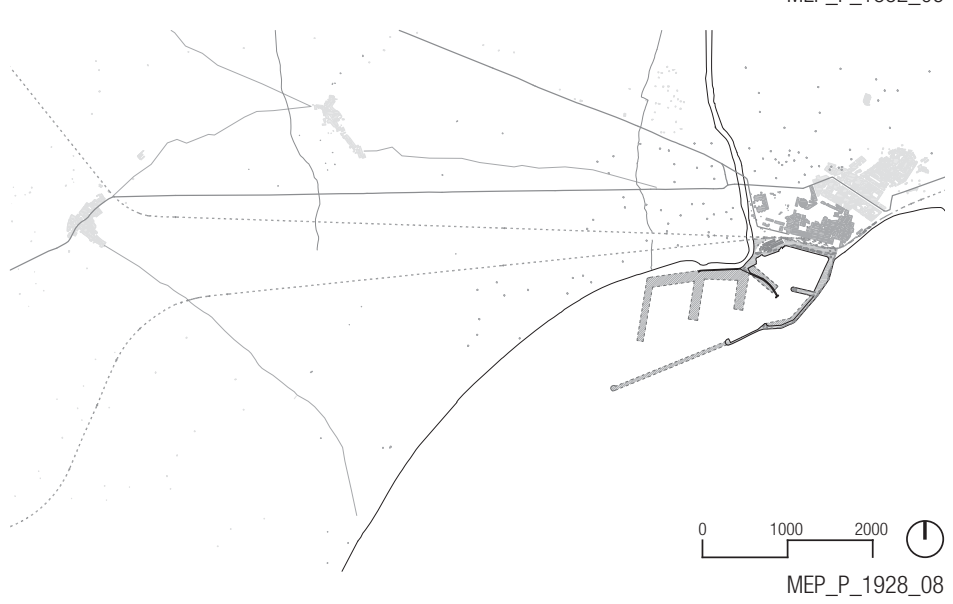




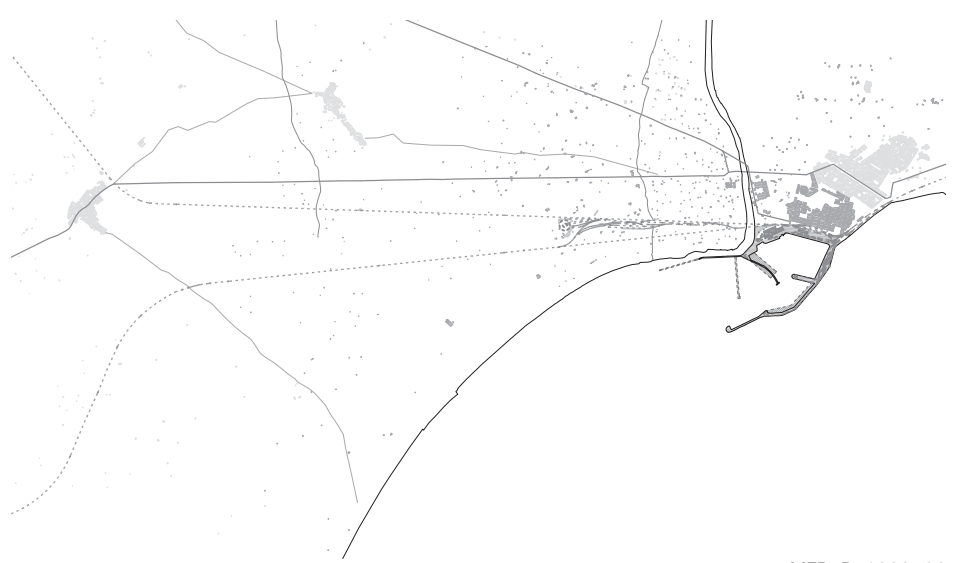

MEP_P_1939_09

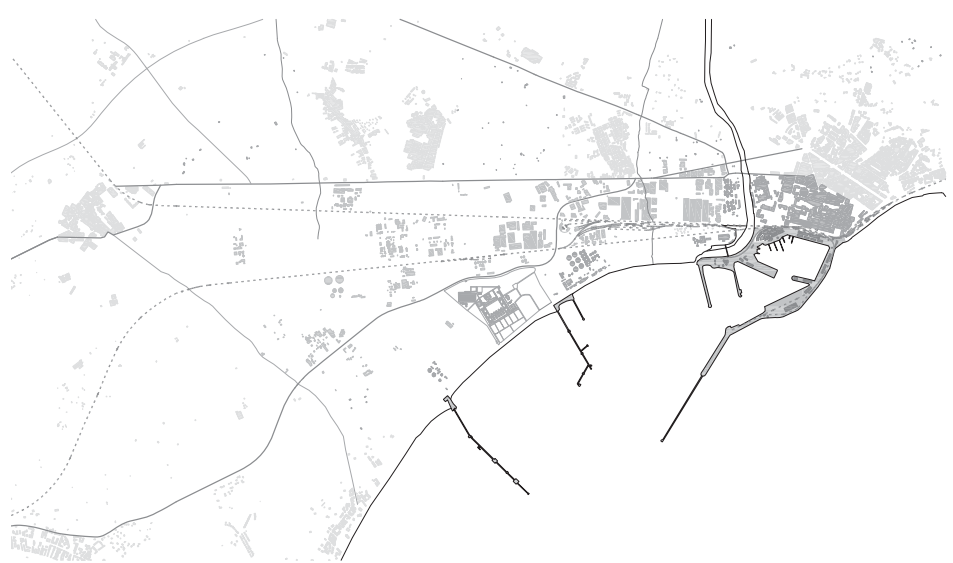

MEP P 1976

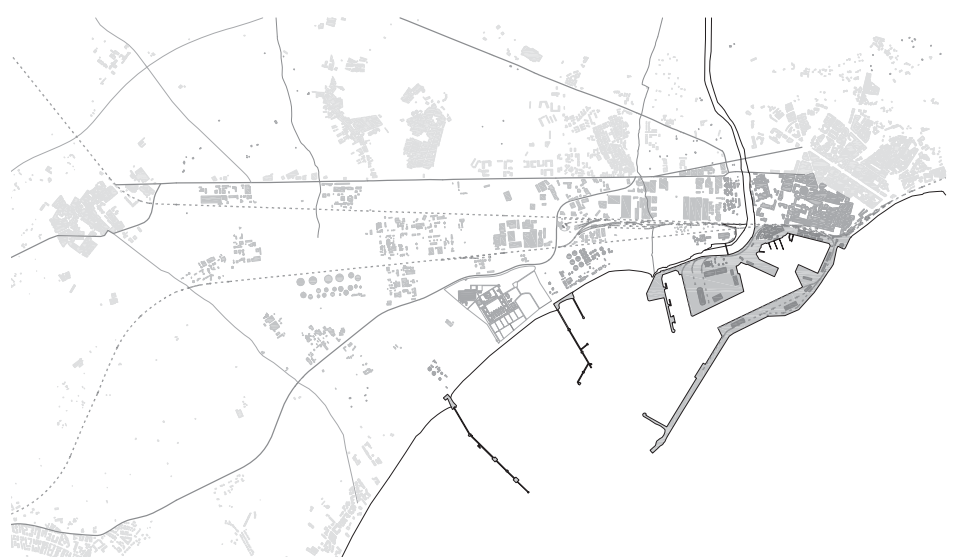

MEP_P_1983_13

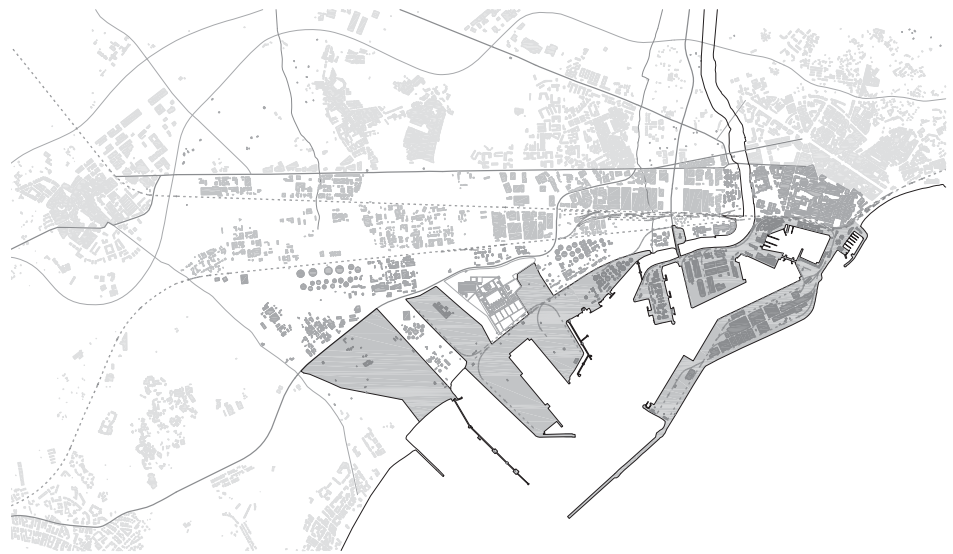

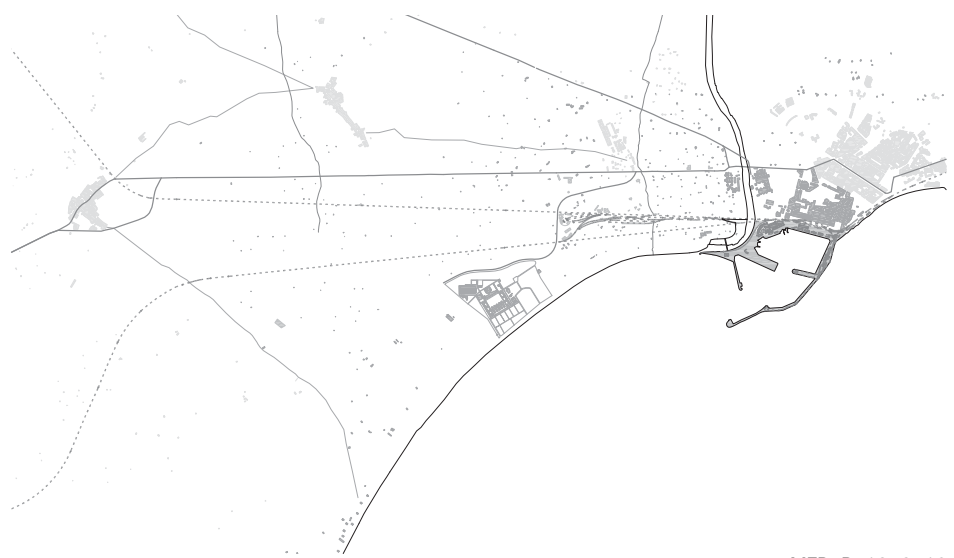

MEP_P_1956_10

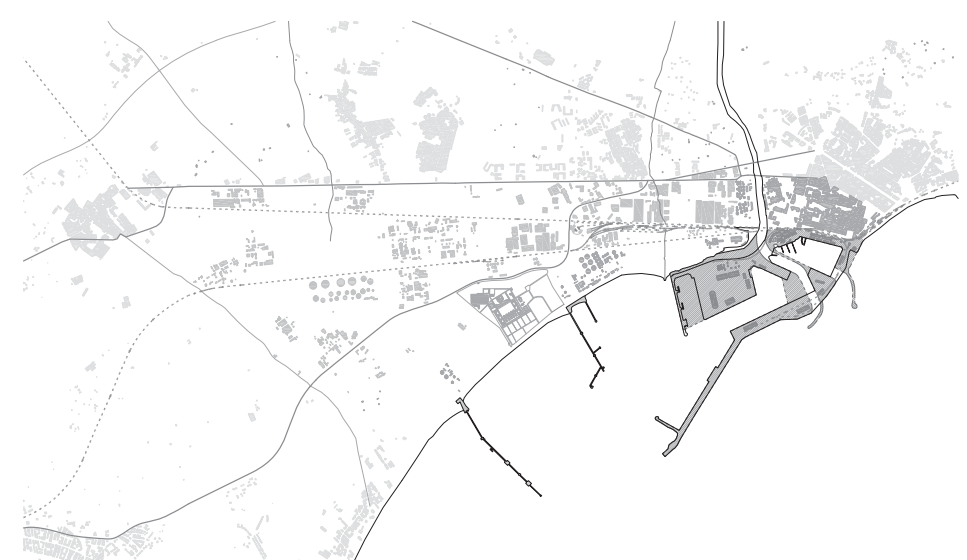

MEP_P_1983P_12

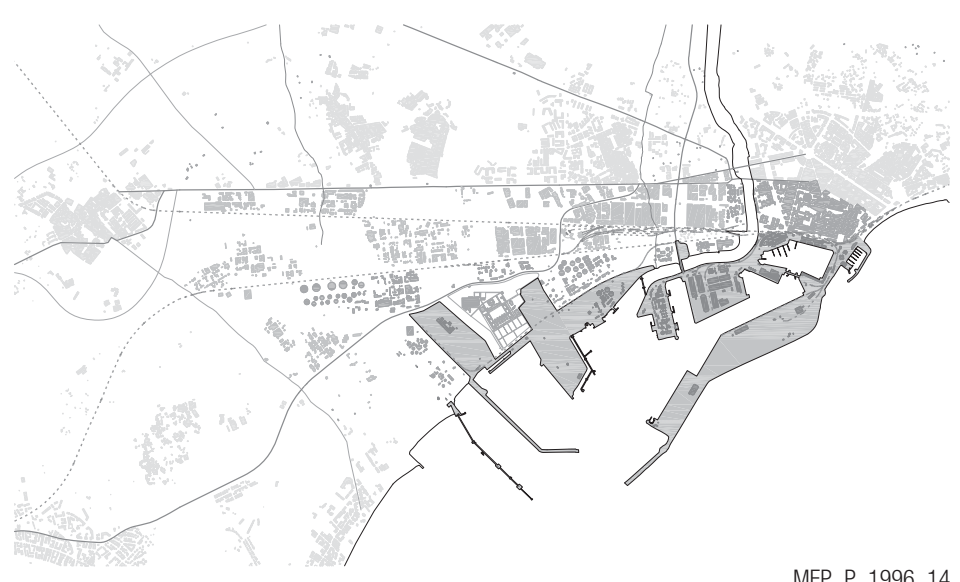

MEP_P_1996_14

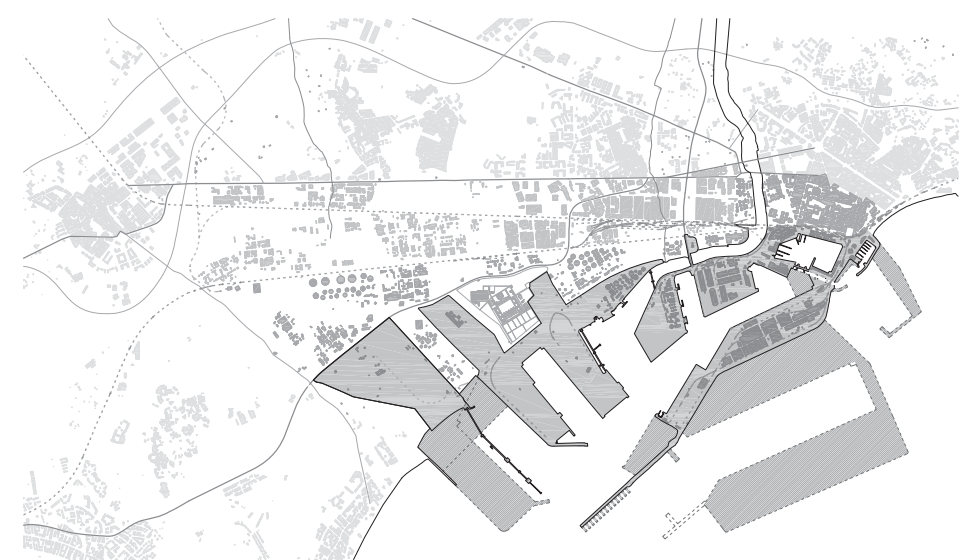

MEP_P_2016_16 

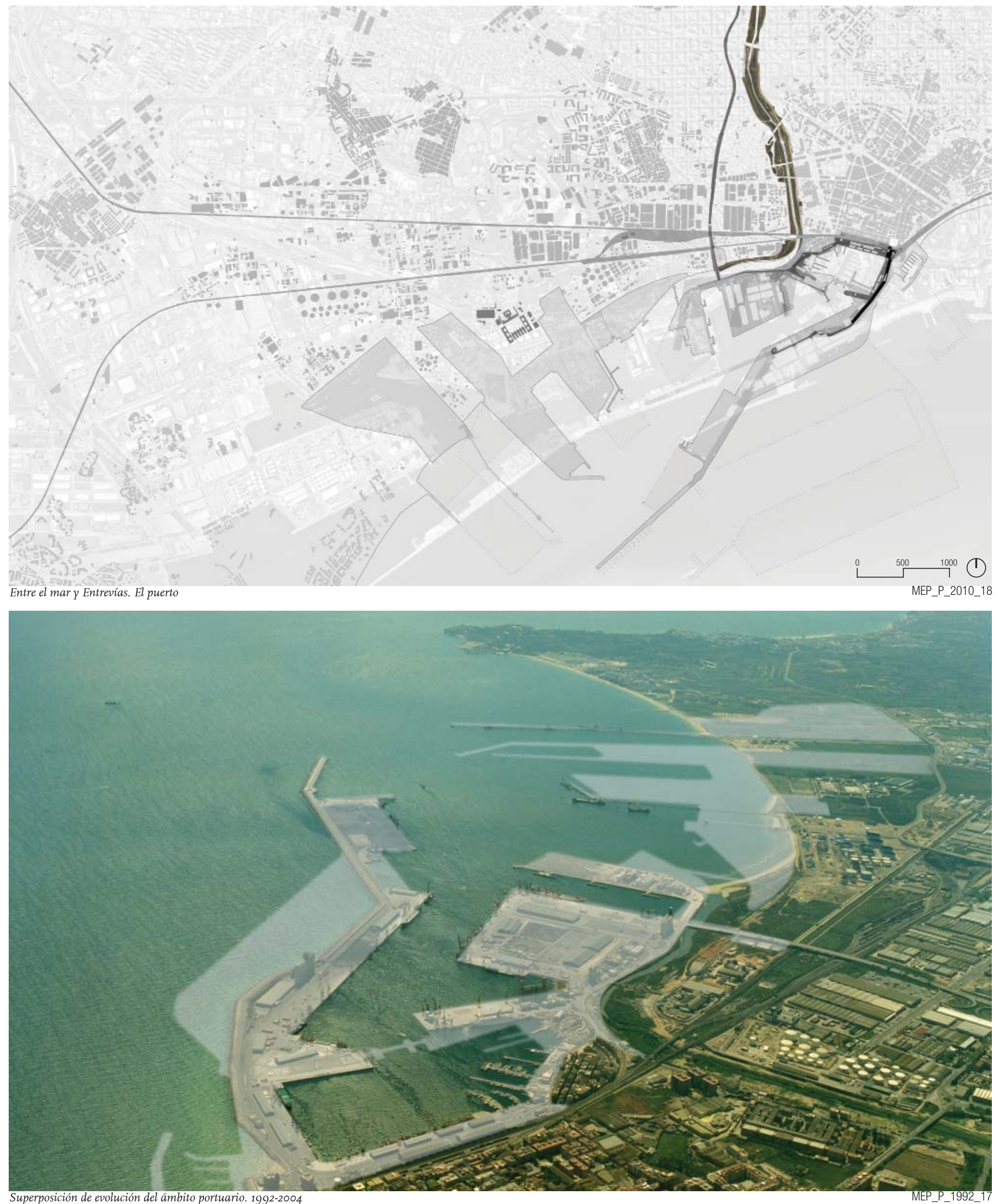

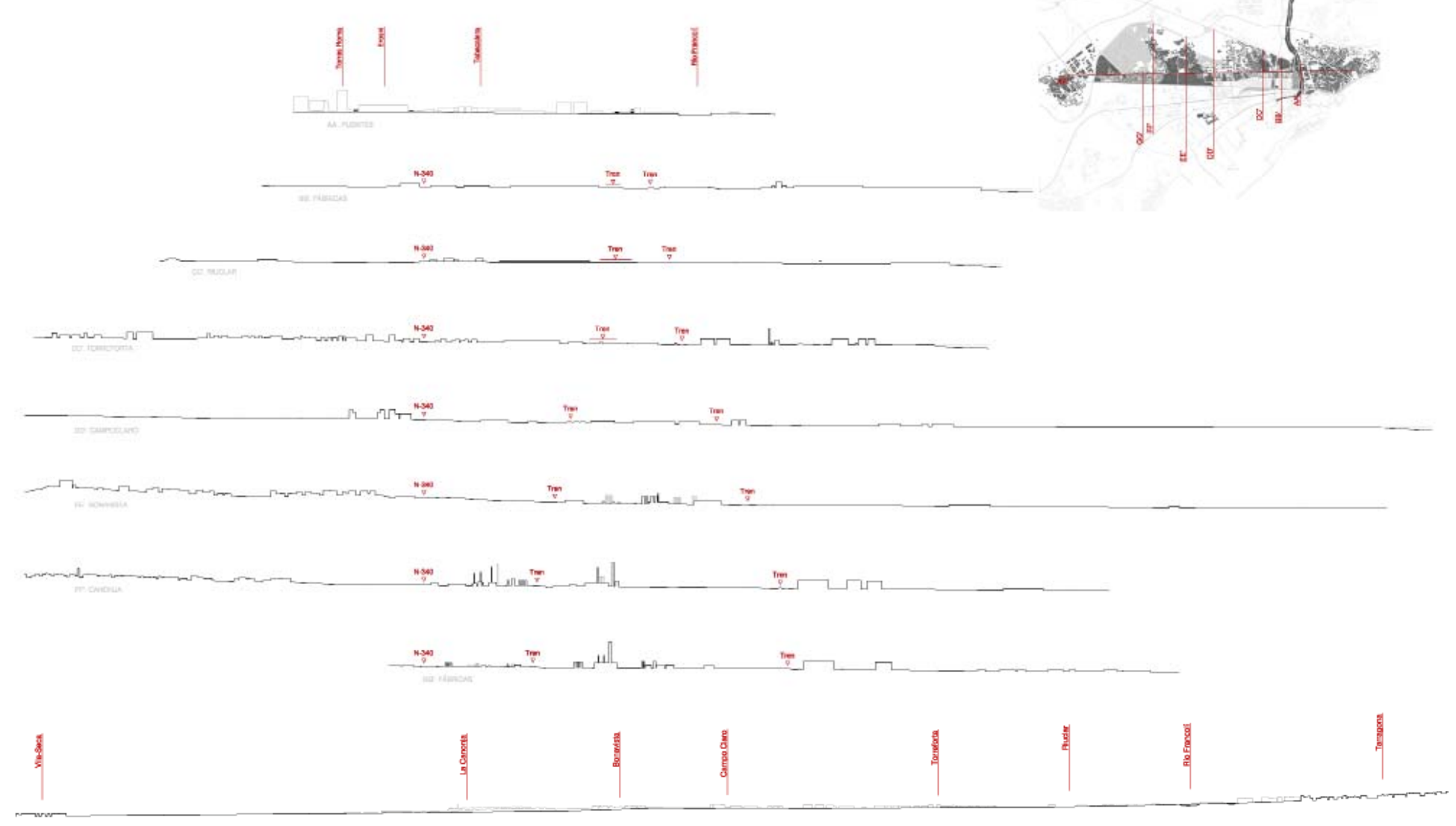

Sección longitudinal por N-340

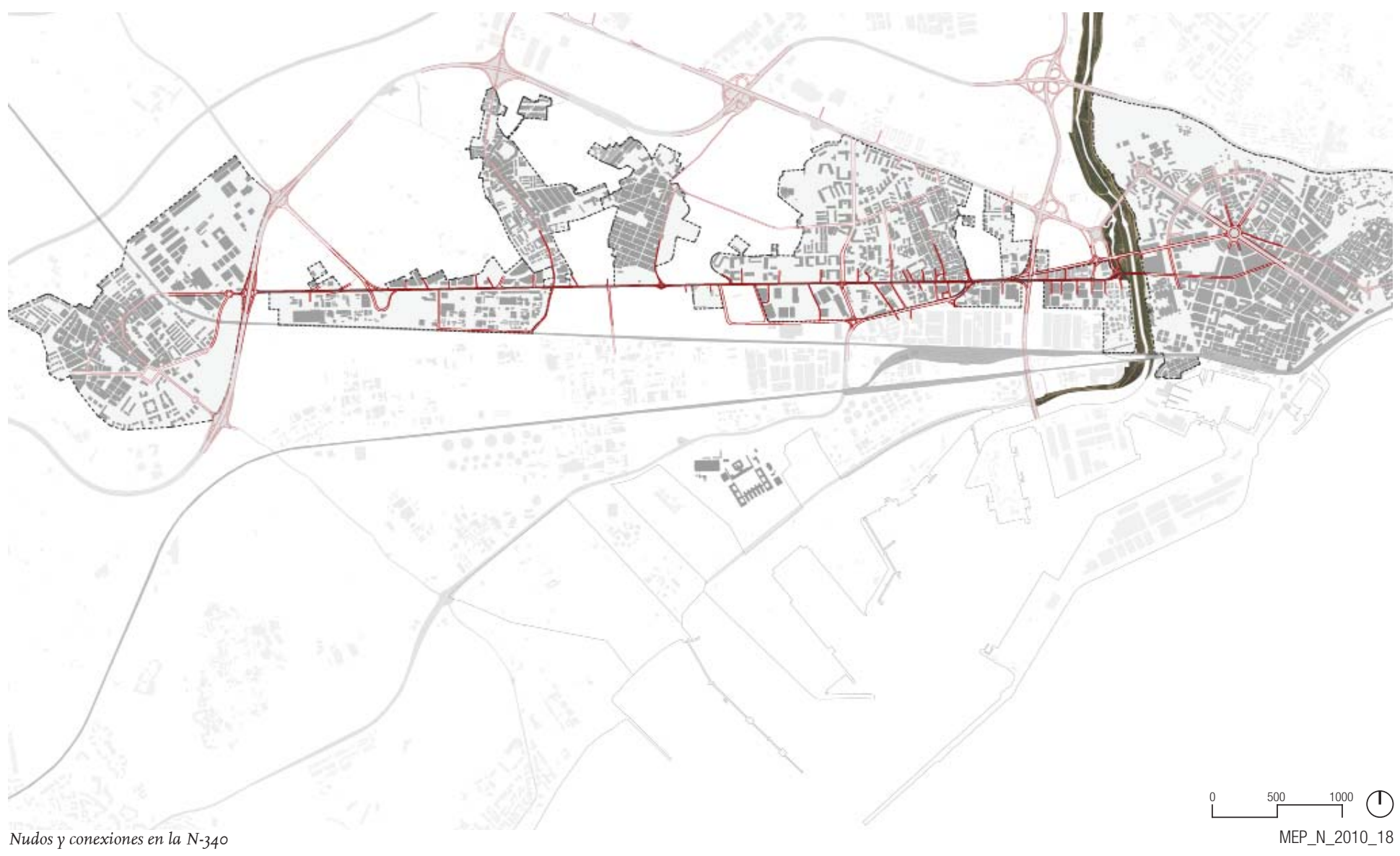



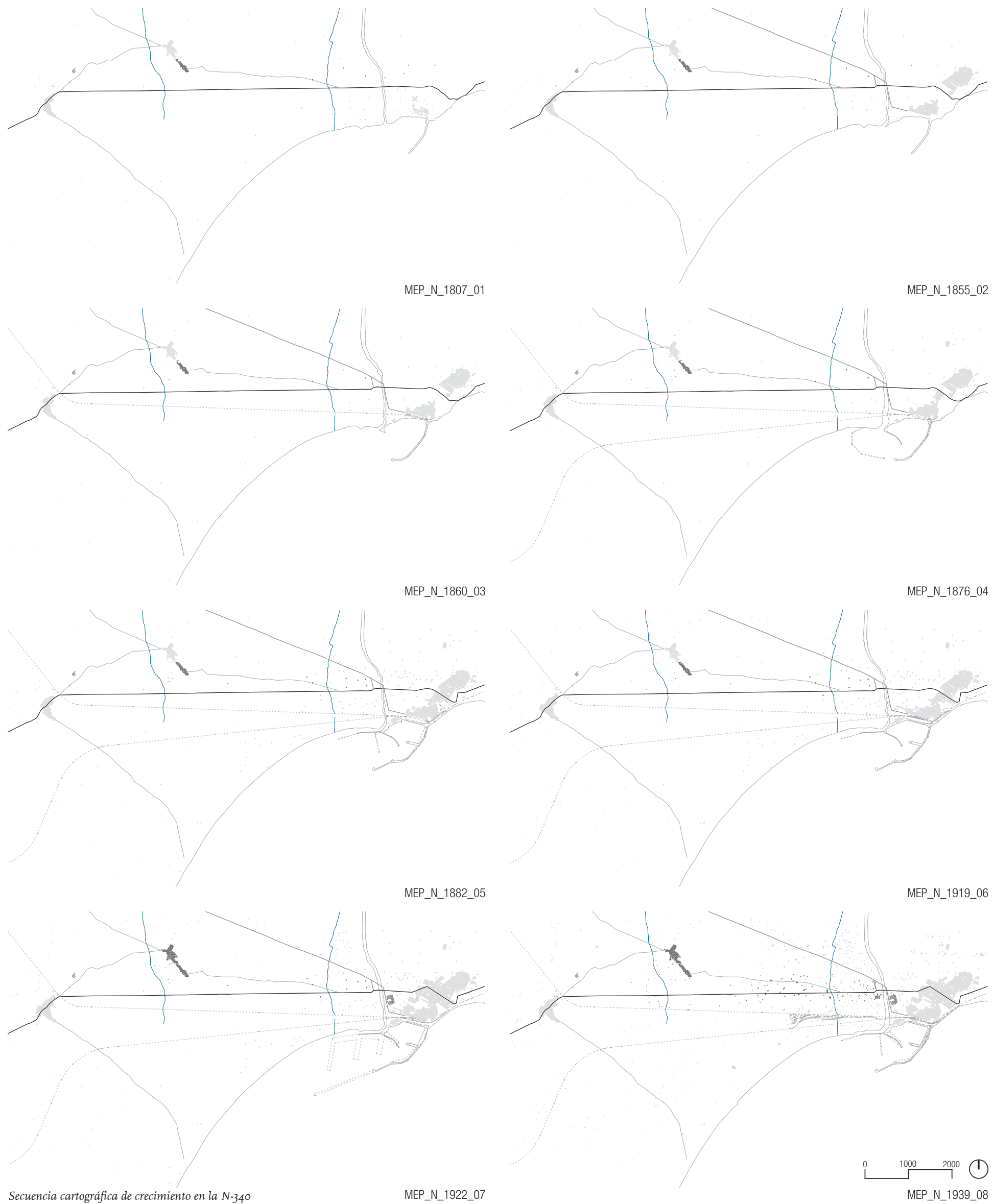


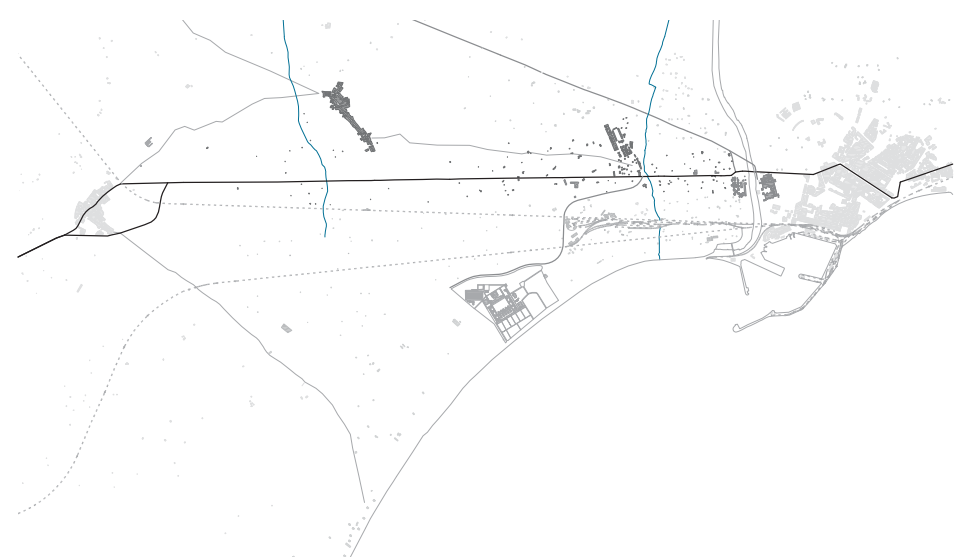

MEP_N_1956_09

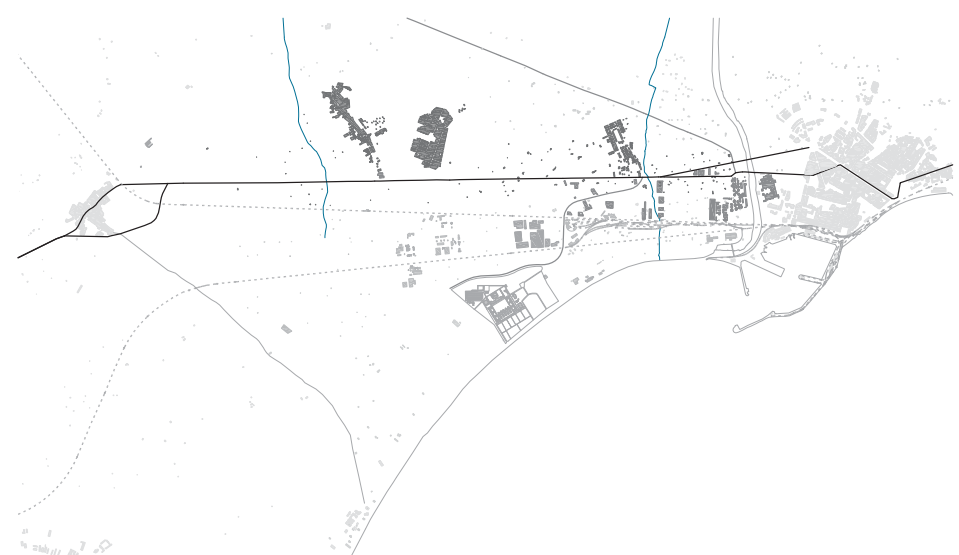

MEP_N_1966_11

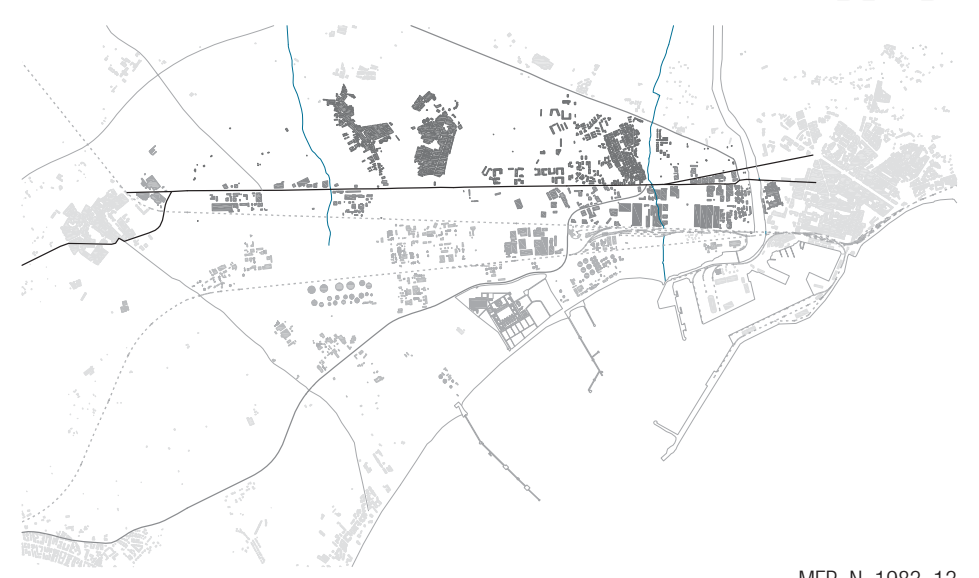

MEP_N_1983_13

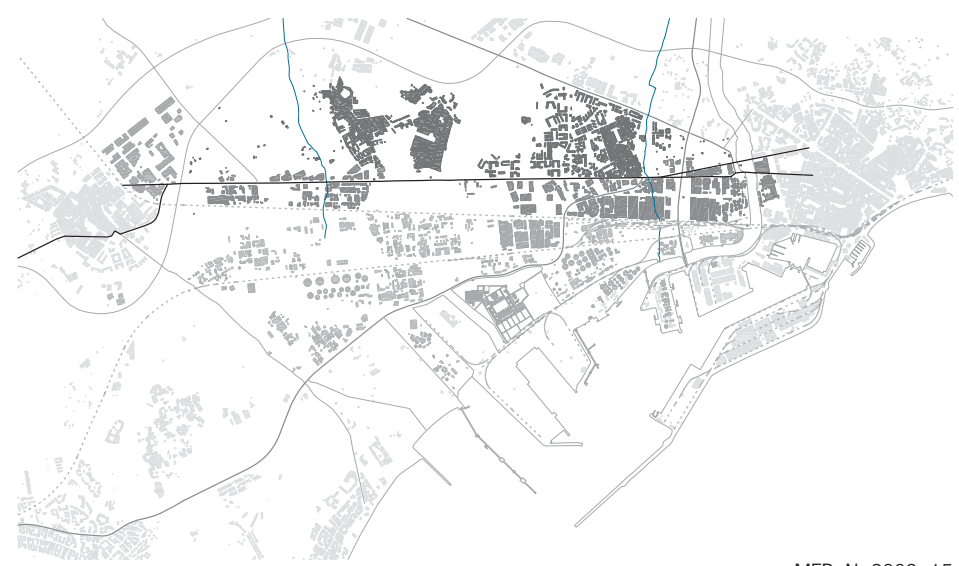

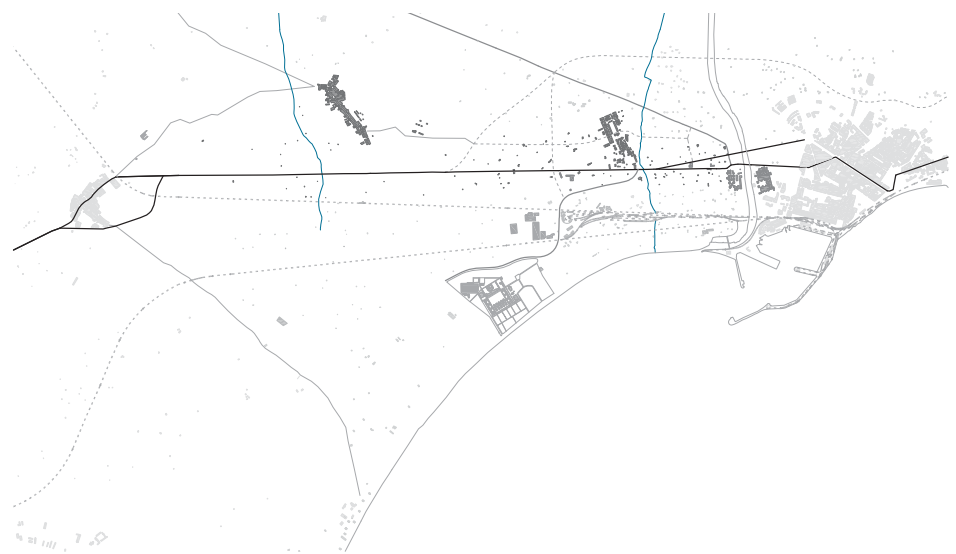

MEP_N_1960_10

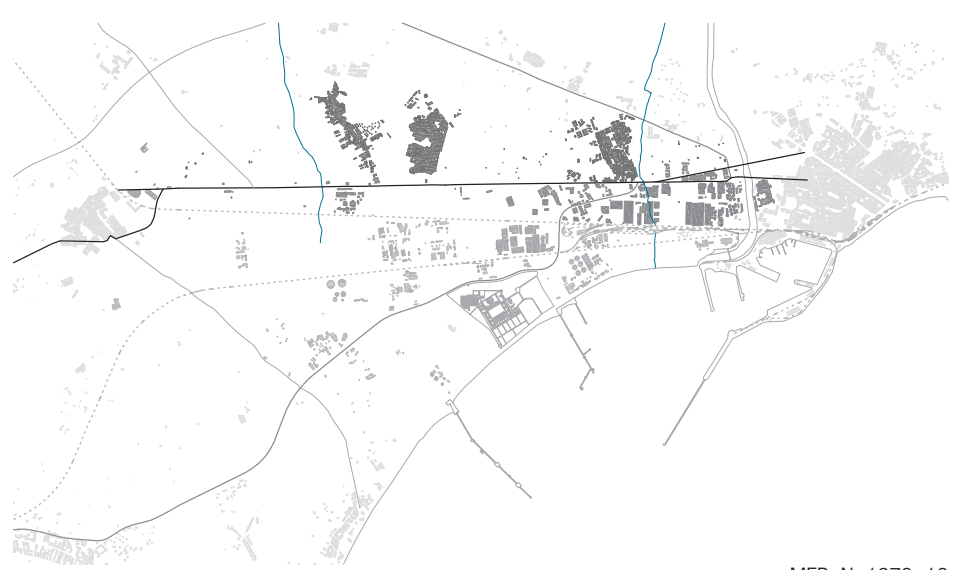

MEP_N_1973_12
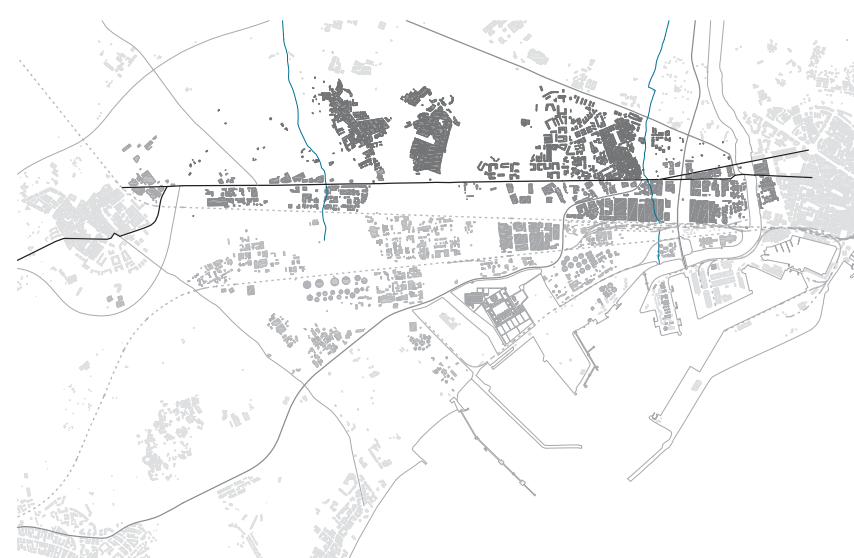

MEP_N_1996_14

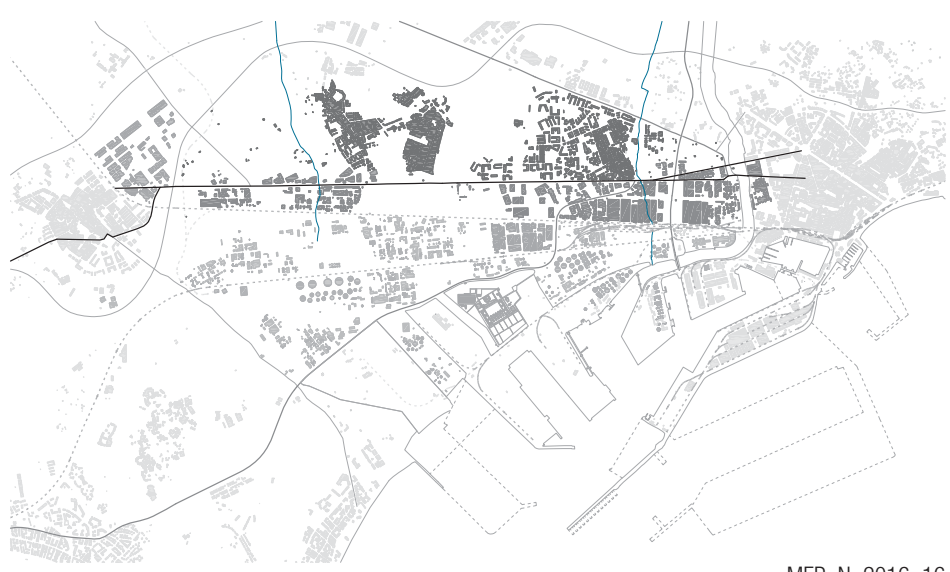

MEP_N_2016_16 

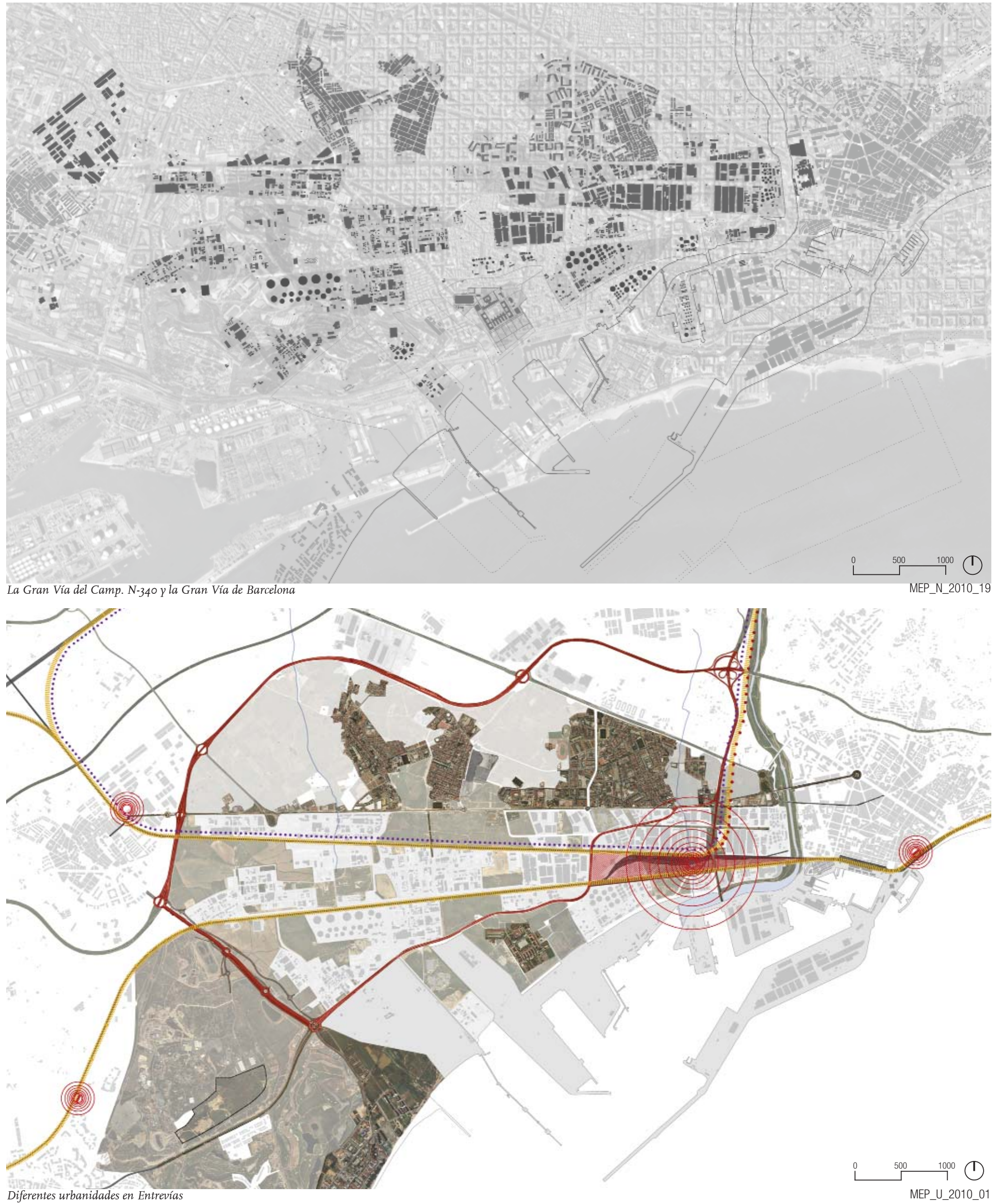

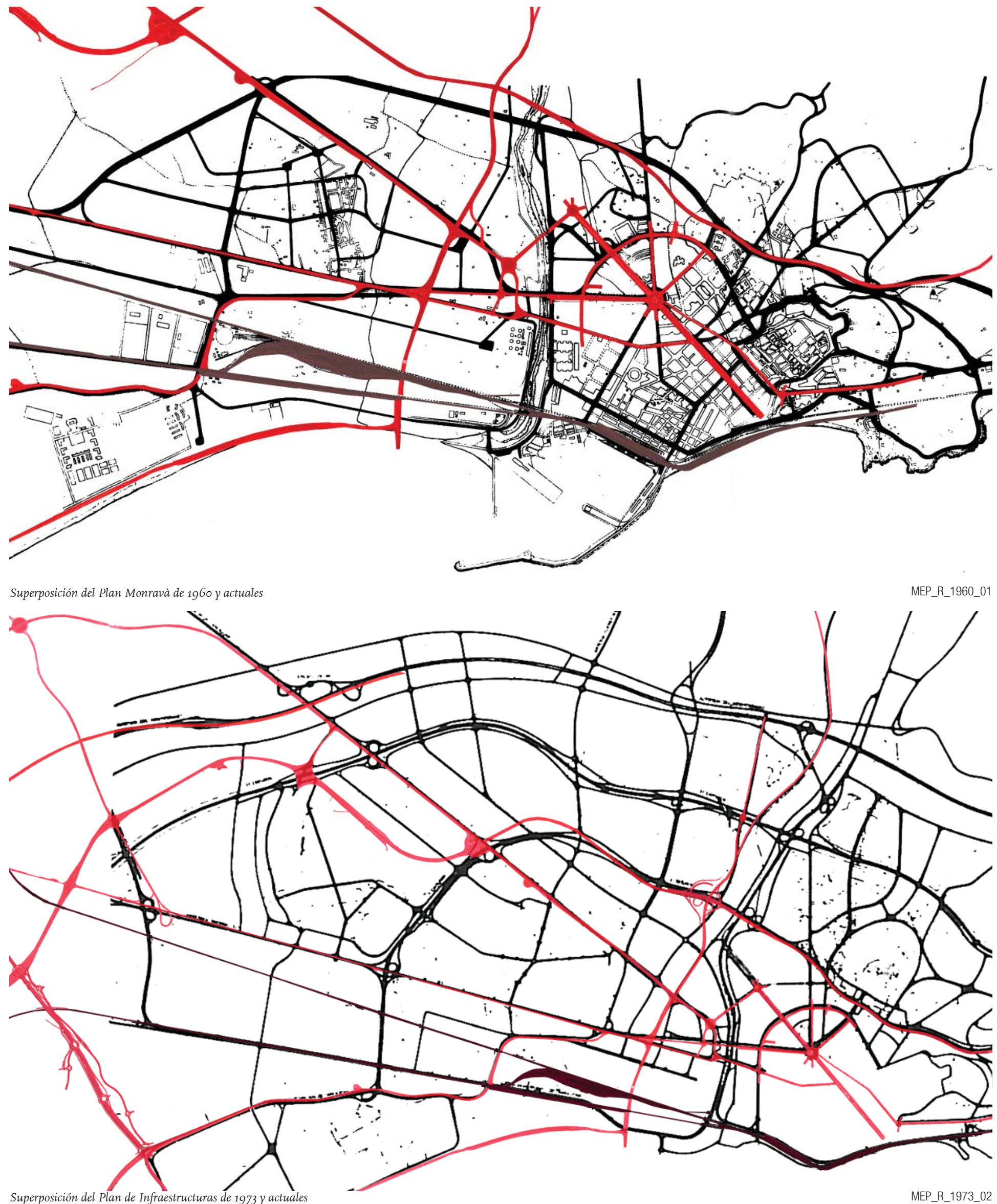

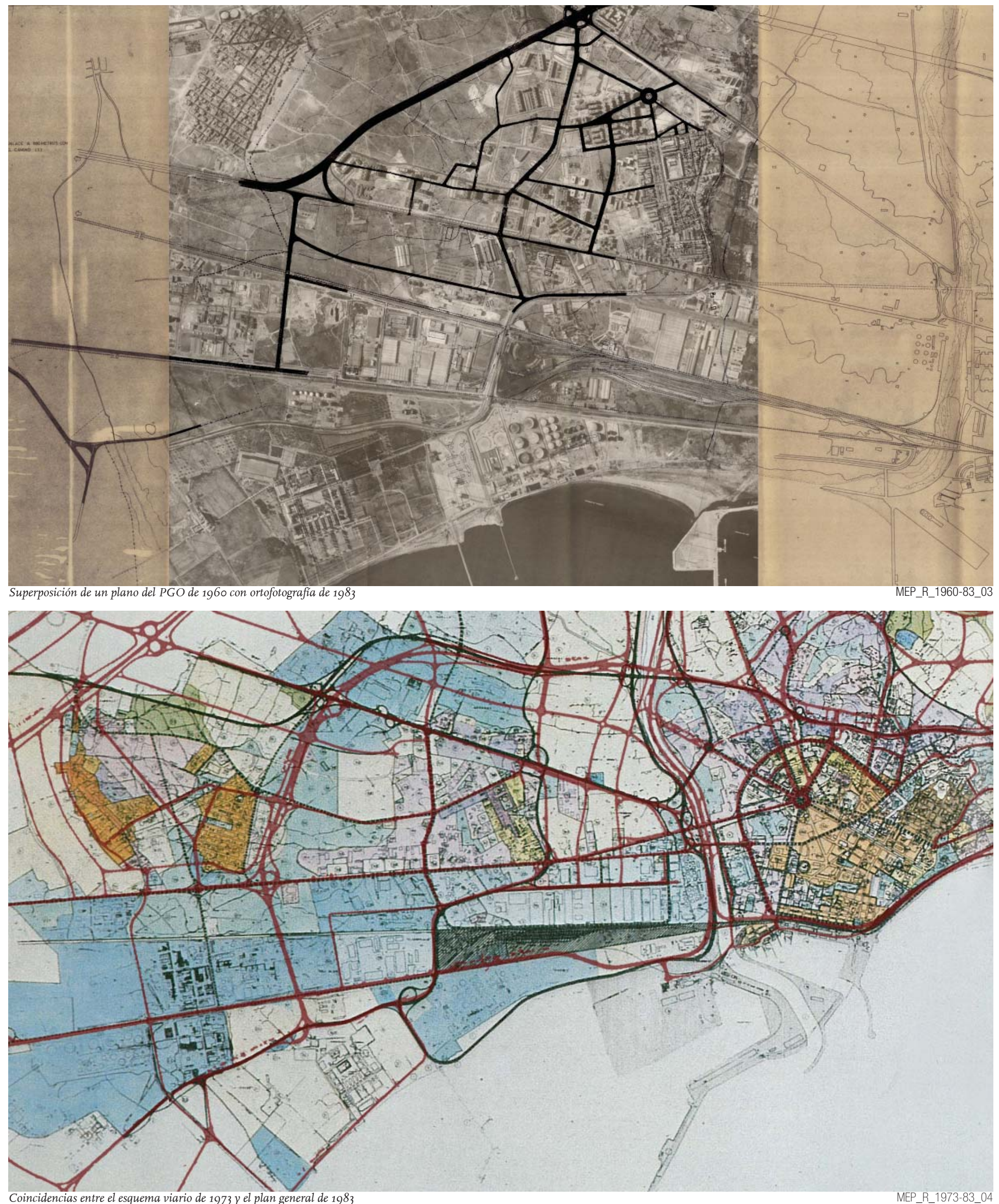

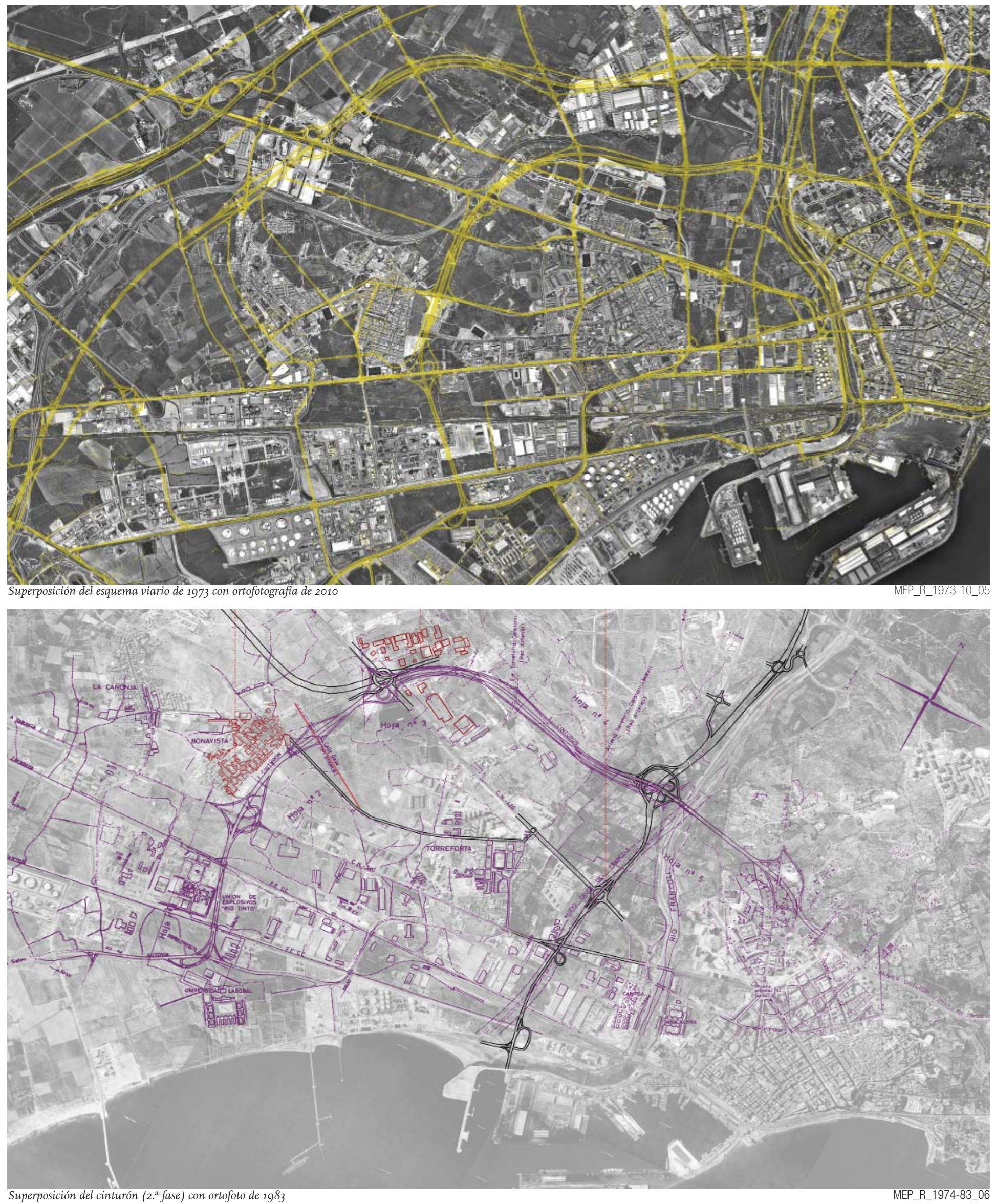



\section{LISTADO DE FUENTES}





\section{Documentación histórica}

AAA Archivo Arquitecto Aragonés

AAAP Archivo Arquitecto Antonio Pujol

AAJMM Archivo Arquitecto J. M. Monravà

AAJMP Archivo Arquitecto J. M. Pujol

AAMA Archivo Arquitecto Manuel Aguilera

AAOB Archivo Arquitecto Oriol Bohigas

AAPM Archivo Arquitecto Pascual Monravà

AAR Archivo Arquitecto Ripoll

AARS Archivo Arquitecto Ramón Salas

AAV Archivo Ayuntamiento de Vila-seca

ACA Archivo Corona de Aragón

ACL Archivo Centro de Lectura

ADGU Archivo Dirección General de Urbanismo

AEQT Asociación Empresarial Química de Tarragona

AFA Archivo Fotográfico Aviotec

AFC Archivo Fotográfico Canadell

AFF Archivo Fotográfico Firtesa

AFG Archivo Fotográfico La Granja

AFR Archivo Fotográfico Reus Mediterrani

AFT Archivo Fotográfico Tarragona

AHAT Archivo Histórico Archidiocesano de Tarragona

AHCBC Archivo Histórico Comarcal del Baix Camp

AHT Archivo Histórico de Tarragona

AIA Archivo Imágenes Antiguas de las Comarcas del Camp

ANC Archivo Nacional Cataluña

APT Archivo Puerto de Tarragona

ATA Archivo Tarragona Antiga

AUCT Archivo Unidad de Carreteras de Tarragona

BNE Biblioteca Nacional de España

COACT Col-legi Arquitectes de Catalunya | Tarragona

EMT Empresa Municipal de Transportes Públicos de Tarragona (Archivo)

HABC Hemeroteca $A B C$

HDT Hemeroteca Diari de Tarragona

HLV Hemeroteca La Vanguardia

ICC Instituto Cartográfico de Cataluña

IGN Instituto Geográfico Nacional

LGM La Gaceta de Madrid

MFE Ministerio de Fomento de España

NODO Noticiero y Documentales | RTVE

RIRI Referencias Internacionales

STUT Servicio Territorial Urbanismo de Tarragona (Archivo)

\section{Documentación de fuentes bibliográficas}

AOJ Atlas Oskar Jurgens | Oskar Jurgen

ARPG Adaptació i revisió del Pla General d'Ordenació Urbana
Informe del Banco Mundial

Els barris d'Adigsa. Campclar | Rosa M Bosch

Historia del Aeropuerto de Reus | Aena

Els barris d'Adigsa. Riuclar | Carmen Luque

Alejandro de la Sota

Els barris d'Adigsa. Torreforta | Carmen Luque

Bonavista. Una biografía social |F. Bajardi

Los Barrios de Tarragona | J. Pujadas y F. Bajardí

Forma urbis. Cinco ciudades bajo sospecha $\mid$ Josep Parcerisa Bundó

La Guía del Camp | COAC Tarragona

Guia-Catàleg de l'Arxiu Fotogràfic | Port de Tarragona

La industrialización de Tarragona $\mid$ Felix Llovell Fortuny. AEQT

LITSC La industrialització de Tarragona i les seves circumstàncies | Josep Llop Tous

LLC La Canonja. El camí a la municipalitat | Ayuntamiento de La Canonja

LRT El renaixement de Tarraco | Lluís Pons d'Icart y Anton Van den Wyngaerde

LTCM Tarragona. El camí cap a la modernitat. Urbanisme i Arquitectura| Elena de Ortueta

LUL La Universitat Laboral| COAC Tarragona

LVA La Via Augusta

NC New Towns

PC Pequeños Congresos

PTTI Proyectar el territorio en tiempos de incertidumbre

PUSB Plan de Urgencia Social de Barcelona

ROP Revista Obras Públicas

TOC Tarragona Ordenación de la Costa

TOT Tarragona. Onomàstica tarragonina amb anotacions multidisciplinars

TPT La Tarjeta Postal a Tarragona. 100 anys d'historia (1897-1996)

UOP El Urbanismo de las Obras Públicas

\section{Documentación planes urbanísticos}

CMC Comisión de Municipios del Camp de Tarragona

PCRTV Plan Comarcal Tarragona-Reus-Valls, ı966

PDUAC Pla Director Urbanístic de l’Àmbit Central del Camp de Tarragona

PDUAI Pla Director Urbanístic de les Activitats Industrial i Turístiques del Camp de Tarragona

PEOCPT Plan Especial de Ordenación de la Costa Provincial de Tarragona

PGO Plan General de Ordenación I960. Plan Monravà

PGOUT Plan General de Ordenación Urbana de Tarragona. Lluís Cantallops i Valeri

PTCT Plan Territorial del Camp de Tarragona

PUDIB Plan de Urgencia de Descongestión Industrial de Barcelona

RPGO Revisión Plan General de Ordenación Tarragona I973

\section{Documentación gráfica elaborada}

MEP Material elaboración propia

RFA Reportaje fotográfico actual 

LISTADO DE ILUSTRACIONES 

Tarragona. Vista desde la torre de San Fructuoso | Anton Wyngaerden Tarragona. Vista desde la torre de San Fructuoso | Anton Wyngaerden Tarragona. Grabado “Tarragone en Catalogne” | Sieur de Beaulieu Tarragona. Plano del corregimiento | Darnius Camp de Tarragona. Plano general | Nicholas Tindal Tarragona. Vista publicada en Atlante Español | Francesc Carreras La Pineda. Plano con ubicación del mas de la Obra | Autor Desconocido Puerto. Plano que comprende la parte baja de la población | Antoni Bada Comunicación Real Orden de 24 de marzo de I80I | Libro actas municipales Comunicación Real Orden de 24 de marzo de I80I | Libro actas municipales Comunicación Real Orden de 24 de marzo de I80I | Libro actas municipales Comunicación Real Orden de 24 de marzo de I80I | Libro actas municipales Comunicación Real Orden de 24 de marzo de I80I | Libro actas municipales Comunicación Real Orden de 24 de marzo de I80I | Libro actas municipales Comunicación Real Orden de 24 de marzo de I80I | Libro actas municipales Comunicación Real Orden de 24 de marzo de I80I | Libro actas municipales Comunicación Real Orden de 24 de marzo de i80 | Libro actas municipales Comunicación Real Orden de 24 de marzo de i80 | Libro actas municipales Comunicación Real Orden de 24 de marzo de i80I | Libro actas municipales Comunicación Real Orden de 24 de marzo de I80I | Libro actas municipales Comunicación Real Orden de 24 de marzo de I80I | Libro actas municipales Comunicación Real Orden de 24 de marzo de I80I | Libro actas municipales Comunicación Real Orden de 24 de marzo de I80I | Libro actas municipales Comunicación Real Orden de 24 de marzo de I80I | Libro actas municipales Puerto. Plano general | Autor Desconocido Puerto. Plano general | Vicente Teixeira Puerto. Proyecto de la población de La Marina | Joan Smith Tarragona.Peña botada del puerto de Tarragona al mar | Alexandre Laborde Tarragona. Plano de la ciudad y nuevo puerto | Alexandre de Laborde Tarragona-Reus-Salou. Plano geográfico del "triángulo" | Autor Desconocido Plano de la nueva población de La Marina | Smith Puerto. Plano general | Ramón Folgueras Puerto. Plano general | Vicente Teixeira Vista del delta Francolí, fortificaciones y acueductos. Grabado siglo xIx | Skelton Tarragona. Plano general | Autor Desconocido Tarragona. Plano general | Laguillermie et Rambox Grabado francés del siglo xix. Vista desde el Fortí de l'Oliva | Marechal Suchet Tarragona. Plano de la parte baja de la ciudad | Marechal Suchet Tarragona. Grabado "Il nemico prende Tarragona” | Bartolomeo Pinelli Tarragona. Plano general | Alexis Barriere Tarragona. Plano de las fortificaciones | Autor Desconocido Gráficos para construcción de carretera $\mid$ Autor Desconocido Gráficos para construcción de carretera | Autor Desconocido
1816_APT_03 1828_ACA_01 1828_ICC_02 1853_ROP_01 1854_LTCM_01 1855_APT_01 1855_LTCM_02 1857_COACT_01 1857_COACT_02 1857 COACT 03 1857_COACT_04 1857_COACT_05 1857_LTCM_06 1860_APT_01 1860_ICC_02 1864_LTCM_01 1865_AFT_01 1865_ICC_02 1865_ICC_03 1865_ICC_04 1865 ICC 05 1865 ICC 06 1865_ICC_07 1865_ICC_08 1865_ICC_09 1868_LTCM_01 1869_LTCM_01 1870_LGF_01 1873_RIRI_01 1876_ICC_01 1879 AFT 01 1880_ATA_01 1880_ATA_02 1880_ICC_03 1882_APT_01 1882_APT_02 1882_COACT_03 1882_COACT_04 1882_LTCM_05 1883_APT_01 1883_LGC_02 1884_AARS_01 1884_COACT_02
Gráficos para construcción de carretera | Autor Desconocido Camp de Tarragona. Plano de caminos, ríos y pueblos | Autor Desconocido Tarragona. Ciudad sitiada por el ejército francés | Adolphe Bossange Ferrocarril Tarragona-Reus. Plano general | J. Donon Tarragona. Plano de la parte baja de la población | Ángel Camón Puerto. Plano general | Autor Desconocido

Ferrocarril Tarragona-Reus. Proyecto de estación | Federico Alameda Tarragona. Plano del ensanche parcial | Pau Monguió Tarragona. Plano del ensanche parcial | Pau Monguió Tarragona. Plano del ensanche parcial | Pau Monguió Tarragona. Plano del ensanche parcial | Pau Monguió Tarragona. Plano del ensanche parcial | Pau Monguió Tarragona. Plano del ensanche parcial | Pau Monguió Puerto. Plano general | Autor Desconocido Camp de Tarragona. Croquis de caminos vecinales | Baudilio Ribot Ferrocarril. Proyecto de estación clasificadora | Tomás Fremor Instantánea de los trabajos en la pedrera cercana al puerto | M. Martínez Proyecto ferrocarril Hospitalet de l'Infant - Tarragona. Tramo I | Autor Desconocido Proyecto ferrocarril Hospitalet de l'Infant - Tarragona. Tramo 2 | Autor Desconocido Proyecto ferrocarril Hospitalet de l'Infant - Tarragona. Tramo $3 \mid$ Autor Desconocido Proyecto ferrocarril Hospitalet de l'Infant - Tarragona. Tramo 4 | Autor Desconocido Proyecto ferrocarril Hospitalet de l'Infant - Tarragona. Tramo 5 | Autor Desconocido Proyecto ferrocarril Hospitalet de l'Infant - Tarragona. Tramo 6 | Autor Desconocido Proyecto ferrocarril Hospitalet de l'Infant - Tarragona. Tramo $7 \mid$ Autor Desconocido Proyecto ferrocarril Hospitalet de l'Infant - Tarragona. Tramo 8 | Autor Desconocido Tarragona. Población entre el baluarte Llatzaret y el rancolí | Francesc d'Arajol Tarragona. Plano general | Manuel de Ortega

Puerto. Vista general $\mid J$. Laurent Belval. Luxemburgo | Autor Desconocido Puerto. Plano general con proyectos | Dirección de Hidrografía Tarragona. Portal del Francolí | Torres Tarragona. Plaza de Els Carros | Autor Desconocido Tarragona. Plaza de Els Carros | Autor Desconocido Plano de inventario de industrias y comercios en Tarragona | Juan Calvet $y$ Boix Tarragona. Plano de la rada y el puerto | Rafael Pardo de Figueroa Tarragona. Plano de la rada y el puerto | Rafael Pardo de Figueroa Tarragona. Emplazamiento de la estación central de ferrocarriles | Autor Desconocido Emplazamiento del proyecto de estación central de ferrocarriles | Autor Desconocido Tarragona. Proyecto para nueva estación central de ferrocarriles | Saturnino Bellido Puerto. Plano con cotas | Autor Desconocido

Salou. Plano de la rada y el cabo | Rafael Pardo de Figueroa Tarragona. Plano de la zona del ensanche | Ramón Salas Tarragona. Plano del barrio de pescadores | Ramón Salas 
1884_LTCM_03 Tarragona. Plano general | Francisco Navarro

1885_COACT_01 Diferentes versiones para la construcción del dique del Oeste | Autor Desconocido

1885_COACT_02 Puerto. Estado actual | Autor Desconocido

1885_COACT_03 Puerto. Obras proyectadas | Autor Desconocido

1885_COACT_04 Puerto. Trazado del dique del Oeste | Ingeniero de las obras

1889_LTCM_01 Puerto. Diseño del muelle Paralelo | Lluís de Corsini

1890_COACT_01 Tarragona. Plano de urbanización de las afueras | Ramón Salas

1890 LTCM 02

1892_LLC_01

1892_LLC_02

1894_LTCM_01

1896_AARS_01

1896_AARS_02

1897_COACT_01

1899_AHT_01

1900_ICC_01

1900_AHT_02

1900_LGF_03

1910_AFT_01

1910_AFT_02

1910_ATA_03

1911_TPT_01

1912_ATA_01

1914_AFT_01

1916 COACT_01

1917_ATA_01

1917_ATA_02

1917_TPT_03

1917_COACT_04

1919_APT_01

1919_TPT_02

1919_TPT_03

1920_AFT_01

1920 AFT 02

1920 AFT 03

1920_ATA_04

1920_COACT_05

1920 COACT 06

1920 ICC 07

1920_ICC_08

1920_IGN_09

1920_IGN_10

1922_AAJMP_01

1922 AAJMP 02

1922_COACT 03

1922_ICC_04

1922_IGN_05

1922_IGN_06

1922_IGN_07

1924_IGN_01

1925_AFT_01

1925_ATA_02

1925 LGF 03

1925 TPT 04

1925_ROP_05

1926_AAJMP_01

Tarragona. Plano de urbanización de las afueras | Ramón Salas

La Canonja. Plano perímetro del término municipal | Autor Desconocido

La Canonja. Plano general | Juan Prats Estela

Tarragona. Plano del ensanche parcial | Pau Monguió

Tarragona. Croquis de la línea del tranvía | Ramón Salas

Puerto. Replanteo del muelle de costa | Fausto Elis Vidarte

Tarragona. Plano general | Ramón Salas

Tarragona. Ilustración de las fortificaciones y murallas | Autor Desconocido

Tarragona. Plano del segundo plan de ensanche | Ramón Salas Ricomá

Tarragona. Plaza de Els Carros | Autor Desconocido

Puerto. Vista general | Autor Desconocido

Tarragona. Plaza de Els Carros y puerto | Chinchilla

Puerto. Bajada del Toro y almacenes de las obras $\mid$ Autor Desconocido

Ferrocarril. Estación del Miracle | Carles Micó Cornado

Instantánea de la plaza de Els Carros (plaza de Oliózaga) | A.T.V.

Ferrocarril. Estación del Miracle | Rafael Vidal

Puerto. Vista general | Liñan

Cros. Plano de los terrenos |J. M. Pujol

Puerto. Submarinos atracados $\mid$ Federic Ballell Maymi

Puerto. Submarinos atracados | Federic Ballell Maymi

Instantánea de los puentes del ferrocarril sobre el río | Autor Desconocido

Tarragona. Plano de la ciudad |J. M. Pujol

Puerto. Plano general | Ingeniero Director

Tarragona. Vista panorámica | Autor Desconocido

Tarragona. Vista panorámica | Autor Desconocido

Puerto. Barco ingles anclado Romney | Autor Desconocido

Tarragona. Puente del ferrocarril sobre el río Francolí | Autor Desconocido

Tarragona. Vista de la estación y bajada del Toro | Autor Desconocido

Puerto. Vista general | Autor Desconocido

Tarragona. Plano de la ciudad y del ensanche | J. M. Pujol

Detalle de la configuración viaria en confluencia con río Francolí| Autor Desconocido

La Canonja. Planimetría | Senén Mateos

La Canonja. Nivelación | Senén Mateos

La Canonja. Plano de trabajos topográficos | Ingeniero jefe

La Canonja. Plano de trabajos topográficos | Ingeniero jefe

|J. M. Pujol

Tarragona. Plano de la ciudad y del ensanche $\mid J$. M. Pujol

Tarragona. Plano de la ciudad y del ensanche | J. M. Pujol

Tarragona. Mapa planimétrico | Instituto Geográfico

Tarragona. Plano de trabajos topográficos | Ingeniero jefe

Tarragona. Plano de trabajos topográficos | Ingeniero jefe

Tarragona. Plano de trabajos topográficos | Ingeniero jefe

Tarragona. Plano de la población | Ingeniero jefe

Tarragona. Vista general desde la playa del Miracle | J. Gaspar

Ferrocarril. Vista de la estación | Gabriel Casas

Puerto. Vista general | Autor Desconocido

Tarragona. Puente ferrocarril de Reus | Autor Desconocido

Ferrocarril. Plano de la estación de Clasificación | Autor Desconocido

290
1926_AAJMP_02 Atlantic Refining Company. Emplazamiento de la compañía |J. M. Pujol 1926_AAJMP_03 Atlantic Refining Company. Plano de la finca adquirida | J. M. Pujol 1926_A0J_04 Tarragona. Plano general | Autor Desconocido

1928_APT_01 Tarragona. Plano del puerto con la ciudad y las playas | Ingeniero Director

1928_APT_02 Puerto. Plano con los proyectos | Autor Desconocido

1928_ATA_03 Proyecto de las casas baratas La Colectiva | Autor Desconocido

1928_COACT_04 Tarragona. Plano de la Mina Enfermería | Josep M Pujol

1928_COACT_05 Tarragona. Plano de la Mina Enfermería | Josep M Pujol

1929_ATA_01 Ferrocarril. Vista de la estación | Josep Gaspar $y$ Serra

1929_ATA_02 Unión Azufrera. Vista de la fábrica | Luciano Martínez

1929_ICC_03 Tarragona. Vista aérea | Josep Gaspary Serra

1929_LGF_04 Puerto. Vista de los tinglados y las vías de tren | Autor Desconocido

1930_COACT_01 Tabacalera. Planos de la fábrica | Autor Desconocido

1930_COACT_02 Tabacalera. Plano de restos arqueológicos | Teniente Coronel Ingenieros

1930_AFT_03 Tabacalera. Vista de la fachada en obras | Autor Desconocido

1930_AFT_04 Tabacalera. Vista de la puerta principal | Autor Desconocido

1930_AFT_05 Tabacalera. Vista de la fachada en obras | Autor Desconocido

1932-42_AHT_01 Catastrón de Tarragona | Geografía y Catastro

1932-42_AHT_02 Catastrón. Ampliación | Geografía y Catastro

1932-42_AHT_C1 Catastrón. La Canonja. Pol. I | Geografía y Catastro

1932-42_AHT_C2 Catastrón. La Canonja. Pol. 2 | Geografía y Catastro

1932-42_AHT_C3 Catastrón. La Canonja. Pol. 3 | Geografía y Catastro

1932-42_AHT_C4 Catastrón. La Canonja. Pol. 4 | Geografía $y$ Catastro

1932-42_AHT_C5 Catastrón. La Canonja. Pol. 5 | Geografía y Catastro

1932-42_AHT_C6 Catastrón. La Canonja. Pol. 6 | Geografía y Catastro

1932-42_AHT_C7 Catastrón. La Canonja. Pol. 7 | Geografía y Catastro

1932-42_AHT_C8 Catastrón. La Canonja. Pol. 8 | Geografía y Catastro

1932-42_AHT_C9 Catastrón. La Canonja. Pol. 9 | Geografía y Catastro

1932-42_AHT_C10 Catastrón. La Canonja. Pol. Io | Geografía y Catastro

1932-42_AHT_C11 Catastrón. La Canonja. Pol. II | Geografía y Catastro

1932-42_AHT_C12 Catastrón. La Canonja. Pol. I2 | Geografía y Catastro 1932-42_AHT_C13 Catastrón. La Canonja. Pol. I3 | Geografía y Catastro 1932-42_AHT_C14 Catastrón. La Canonja. Pol. I4 | Geografía y Catastro 1932-42_AHT_C15 Catastrón. La Canonja. Pol. I5 | Geografía y Catastro 1932-42_AHT_C16 Catastrón. La Canonja. Pol. I6 | Geografía y Catastro 1932-42_AHT_C17 Catastrón. La Canonja. Pol. I7 | Geografía y Catastro 1932-42_AHT_R14 Catastrón. Reus. Pol. I4 | Geografía Y Catastro 1932-42_AHT_R15 Catastrón. Reus. Pol.I5 | Geografía y Catastro 1932-42_AHT_R19 Catastrón. Reus. Pol. I9 | Geografía y Catastro 1932-42_AHT_R20 Catastrón. Reus. Pol. 20-23 | Geografía y Catastro 1932-42_AHT_T14 Catastrón. Tarragona. Pol. I4 | Geografía y Catastro 1932-42_AHT_T15 Catastrón. Tarragona. Pol. I5 | Geografía y Catastro 1932-42_AHT_T16 Catastrón. Tarragona. Pol. I6 | Geografía $y$ Catastro 1932-42_AHT_T17A Catastrón. Tarragona. Pol. I7A | Geografía y Catastro 1932-42_AHT_T17B Catastrón. Tarragona. Pol. I7B | Geografía y Catastro 1932-42_AHT_T18 Catastrón. Tarragona. Pol. I8 | Geografía y Catastro 1932-42_AHT_T19 Catastrón. Tarragona. Pol. I9 | Geografía y Catastro 1932-42_AHT_T20 Catastrón. Tarragona. Pol. 20 | Geografía $y$ Catastro 1932-42_AHT_T21 Catastrón. Tarragona. Pol. 2I | Geografía Y Catastro 1932-42_AHT_T22 Catastrón. Tarragona. Pol. 22 | Geografía Y Catastro 1932-42_AHT_T23 Catastrón. Tarragona. Pol. 23 | Geografía y Catastro 1932-42_AHT_T24 Catastrón. Tarragona. Pol. 24 | Geografía y Catastro 1932-42_AHT_T25A Catastrón. Tarragona. Pol. 25A | Geografía y Catastro 1932-42_AHT_T25B Catastrón. Tarragona. Pol. 25B | Geografía Y Catastro 1932-42_AHT_T26A Catastrón. Tarragona. Pol. 26A | Geografía Y Catastro 1932-42_AHT_T26B Catastrón. Tarragona. Pol. 26B | Geografía $y$ Catastro 
1932-42_AHT_T27 Catastrón. Tarragona. Pol. 27 | Geografía y Catastro 1932-42_AHT_T28 Catastrón. Tarragona. Pol. 28 | Geografía y Catastro 1932-42_AHT_T29 Catastrón. Tarragona. Pol. 29 | Geografía y Catastro 1932-42_AHT_T30 Catastrón. Tarragona. Pol. 30 | Geografía y Catastro 1932-42_AHT_T31 Catastrón. Tarragona. Pol. 3I | Geografía y Catastro 1932-42_AHT_T32 Catastrón. Tarragona. Pol. 32-33 | Geografía y Catastro 1932-42_AHT_T34 Catastrón. Tarragona. Pol. 34 | Geografía $y$ Catastro 1932-42_AHT_T35 Catastrón. Tarragona. Pol. 35 | Geografía y Catastro 1932-42_AHT_T36A Catastrón. Tarragona. Pol. 36A | Geografía $Y$ Catastro 1932-42_AHT_T36B Catastrón. Tarragona. Pol. 36B | Geografía $y$ Catastro 1932-42_AHT_V5 Catastrón. Vila-seca. Pol. 5 | Geografía Y Catastro 1932-42_AHT_V6 Catastrón. Vila-seca. Pol. 6 | Geografía y Catastro 1932-42_AHT_V7 Catastrón. Vila-seca. Pol. 7 | Geografía y Catastro 1932-42_AHT_V12 Catastrón. Vila-seca. Pol. I2 | Geografía y Catastro 1932-42_AHT_V13 Catastrón. Vila-seca. Pol. I3 | Geografía y Catastro 1932-42_AHT_V14 Catastrón. Vila-seca. Pol. I4 | Geografía y Catastro 1932-42_AHT_V15 Catastrón. Vila-seca. Pol. I5 | Geografía y Catastro 1932-42_AHT_V16 Catastrón. Vila-seca. Pol. I6 | Geografía y Catastro 1932-42_AHT_V17 Catastrón. Vila-seca. Pol. I7 | Geografía y Catastro 1934_AFT_01 Tarragona. Vista aérea | Autor Desconocido 1934_ATA_02 Imagen de las casas baratas La Colectiva | Familia Pié 1935_ATA_01 Tarragona. Playa del Miracle | Rafael Vidal 1936_ATA_01 Puerto. Vista hacia la ciudad | Rafael Vidal 1936_ICC_02 Tarragona. Mapa topográfico. Edición guerra civil | Autor Desconocido 1937_HDT_01_Imagen de los bombardeos sobre Campsa | AHT 1938_AHT_01 Tarragona. Vista aérea de los bombardeos | Autor Desconocido 1939_APT_01 Tarragona, la costa y sus entrevías | Autor Desconocido 1939_APT_02 1939_APT_03 1939_APT_04 1939_APT_05 1939_APT_06 1939_COACT_07 1939_COACT_08 1939_COACT_09 1939_COACT_10 1939_COACT_11 1939_COACT_12 1939_COACT_13 1939_COACT_14 1939_COACT_15 1939_COACT_16 1939_COACT_17 1940_AFT_01 1940_LAH_02 1941_COACT_01 1942_ATA_01 1942_ATA_02 1942_COACT_03 1942_COACT_04 1942_COACT_05 1942_COACT_C1 1942_COACT_C2 1942_COACT_C3 1942 COACT C4 1942_COACT_C5
Tarragona. Vista aérea de los bombardeos | Autor Desconocido Puerto. Plano general | Ingeniero Director Puerto. Plano con obras en proyecto | Ingeniero Director Puerto. Plano con obras en proyecto | Ingeniero Director Puerto. Plano con obras en proyecto | Ingeniero Director Cepasa. Plano de situación | Autor Desconocido Cepasa. Emplazamiento | Autor Desconocido Cepasa. Plano de la fachada principal | Autor Desconocido Cepasa. Plano de la fachada lateral | Autor Desconocido Cepasa. Sección transversal | Autor Desconocido Cepasa. Planta | Autor Desconocido Cepasa. Planta | Autor Desconocido Cepasa. Planta estructura | Autor Desconocido Cepasa. Planta estructura | Autor Desconocido Cepasa. Planta estructura | Autor Desconocido Cepasa. Alzado valla | Autor Desconocido Tarragona. Vista general | Autor Desconocido Aeropuerto. Vista de los campos de vuelo | AENA

Cepasa. Vista aérea | Autor Desconocido Obras de modernización del ferrocarril Reus-Tarragona | Rafael Vidal Tarragona. Puente del Francolí| Marin Chivite

Tarragona. Plano general | I.G.C. Plano topográfico parcelario. Zoom | I.G.C. Plano topográfico parcelario. Plano general | I.G.C. Plano topográfico parcelario. La Canonja. Pol. I| I.G.C. Plano topográfico parcelario. La Canonja. Pol. 2 | I.G.C. Plano topográfico parcelario. La Canonja. Pol. 3 | I.G.C. Plano topográfico parcelario. La Canonja. Pol. 4 | I.G.C. Plano topográfico parcelario. La Canonja. Pol. 5 | I.G.C.
1942_COACT_C6 Plano topográfico parcelario. La Canonja. Pol. 6 | I.G.C. 1942_COACT_C7 Plano topográfico parcelario. La Canonja. Pol. 7 | I.G.C. 1942_COACT_C8 Plano topográfico parcelario. La Canonja. Pol. 8 | I.G.C. 1942_COACT_C9 Plano topográfico parcelario. La Canonja. Pol. 9 | I.G.C. 1942_COACT_C10 Plano topográfico parcelario. La Canonja. Pol. Io| I.G.C. 1942_COACT_C11 Plano topográfico parcelario. La Canonja. Pol. II | I.G.C. 1942_COACT_C12 Plano topográfico parcelario. La Canonja. Pol. I2 | I.G.C. 1942_COACT_C13 Plano topográfico parcelario. La Canonja. Pol. I3 | I.G.C. 1942_COACT_C14 Plano topográfico parcelario. La Canonja. Pol. I4 | I.G.C. 1942_COACT_C15 Plano topográfico parcelario. La Canonja. Pol. I5 | I.G.C. 1942_COACT_C16 Plano topográfico parcelario. La Canonja. Pol. i6| I.G.C. 1942_COACT_C17 Plano topográfico parcelario. La Canonja. Pol. I7 | I.G.C. 1942_COACT_R14 Plano topográfico parcelario. Reus. Pol. I4 | I.G.C. 1942_COACT_R15 Plano topográfico parcelario. Reus. Pol. I5 | I.G.C. 1942_COACT_R19 Plano topográfico parcelario. Reus. Pol. I9| I.G.C. 1942_COACT_R20 Plano topográfico parcelario. Reus. Pol. 20 | I.G.C. 1942_COACT_T14 Plano topográfico parcelario. Tarragona. Pol. I4 | I.G.C. 1942_COACT_T15 Plano topográfico parcelario. Tarragona. Pol. I5 | I.G.C. 1942_COACT_T16 Plano topográfico parcelario. Tarragona. Pol. I6 | I.G.C. 1942_COACT_T17A Plano topográfico parcelario. Tarragona. Pol. I7A | I.G.C. 1942_COACT_T17B Plano topográfico parcelario. Tarragona. Pol. i7B | I.G.C. 1942_COACT_T17C Plano topográfico parcelario. Tarragona. Pol. i7C | I.G.C. 1942_COACT_T18 Plano topográfico parcelario. Tarragona. Pol. I8 | I.G.C. 1942_COACT_T19 Plano topográfico parcelario. Tarragona. Pol. I9| I.G.C. 1942_COACT_T20 Plano topográfico parcelario. Tarragona. Pol. $20 \mid$ I.G.C. 1942_COACT_T21 Plano topográfico parcelario. Tarragona. Pol. 2I | I.G.C. 1942_COACT_T22 Plano topográfico parcelario. Tarragona. Pol. 22 | I.G.C. 1942_COACT_T23 Plano topográfico parcelario. Tarragona. Pol. 23 | I.G.C. 1942_COACT_T24 Plano topográfico parcelario. Tarragona. Pol. 24 | I.G.C. 1942_COACT_T25 Plano topográfico parcelario. Tarragona. Pol. 25 | I.G.C. 1942_COACT_T26A Plano topográfico parcelario. Tarragona. Pol. 26A | I.G.C. 1942_COACT_T26B Plano topográfico parcelario. Tarragona. Pol. 26B | I.G.C. 1942_COACT_T27 Plano topográfico parcelario. Tarragona. Pol. 27 | I.G.C. 1942_COACT_T30 Plano topográfico parcelario. Tarragona. Pol. $30 \mid$ I.G.C. 1942_COACT_V5 Plano topográfico parcelario. Vila-seca. Pol. 5 | I.G.C. 1942_COACT_V6 Plano topográfico parcelario. Vila-seca. Pol. 6 | I.G.C. 1942_COACT_V7 Plano topográfico parcelario. Vila-seca. Pol. 7 | I.G.C. 1942_COACT_V12 Plano topográfico parcelario. Vila-seca. Pol. I2 | I.G.C. 1942_COACT_V13 Plano topográfico parcelario. Vila-seca. Pol. I3 | I.G.C. 1942_COACT_V14 Plano topográfico parcelario. Vila-seca. Pol. I4 | I.G.C. 1942_COACT_V15 Plano topográfico parcelario. Vila-seca. Pol. I5 | I.G.C. 1942_COACT_V16 Plano topográfico parcelario. Vila-seca. Pol. I6 | I.G.C. 1942_COACT_V17 Plano topográfico parcelario. Vila-seca. Pol. I7| I.G.C. 1943_APT_01 Puerto. Vista aérea | Autor Desconocido 1945_AFT_01_Casa Bloc. Construcción de las viviendas | Vallvé 1945_AFT_02 Casa Bloc. Construcción de las viviendas | Vallvé 1945_AFT_03 Casa Bloc. Construcción de las viviendas | Vallvé 1945_AFT_04 Casa Bloc. Construcción de las viviendas | Vallvé 1945_AFT_05 Casa Bloc. Construcción de las viviendas | Vallvé 1945_TPT_06 Escuela de Trabajo. Vista general | Autor Desconocido 1946_AFT_01 Tarragona. Vista aérea | Autor Desconocido

1946_NODO_02 Tarragona. Inauguración del nuevo cuartel G Contreras | RTVE 1947_APT_01 Tarragona. Vista aérea | Autor Desconocido 1949_AFT_01 Tarragona. Vista del puente sobre el Francolí | Vallvé 1949_AFT_02 Tarragona. Vista del puente sobre el Francolí| Vallvé 1949_ICC_03 La Fábrica de Tabacos y Campsa. Vista aérea | CETFA 
1950_AFR_01 Pineda de Vilafortuny | Autor Desconocido

1950_AFT_02 Casa Bloc. Maqueta de las viviendas | Vallvé

1950_AFT_03 Casa dels Mestres. Maqueta de las viviendas | Vallvé

1950_AFT_04 Tarragona. Proyecto del cuartel militar de la avenida de Cataluña | Vallvé

1950_AFT_05

1950_AFT_06

1950_ATA_07

1950_TOT_08

1950_AFT_09

1950_AFT_10

1950_AFT_11

1950_AFT_12

1950_COACT_13

1950_COACT_14

1950_COACT_15

1951_AFT_01

1951_AFT_02

1951_ATA_03

1952_AFT_01

1952_AFT_02

1952_APT_03

1952_COACT_04

1952_LUL_05

1953_LAT_01

1954_AFC_01

1954_AFT_02

1954_AFT_03

1954_AFT_04

1954_AFT_05

1954_AFT_06

1954_AFT_07

1954_ATA_08

1954_ATA_09

1954_ATA_10

1954_ATA_11

1954_ATA_12

1954_ATA_13

1954 ATA 14

1954_ATA_15

1954_ATA_16

1954_ATA_17

1954 ATA 18

1954_ATA_19

1954_COACT_20

1954_COACT_21

1954_COACT_22

1954_COACT_23

1954_COACT_24

1954_NODO_25

1954_TPT_26

1954_LAT_27

1955_AFT_01

Prisión. Vista general de la construcción | Vallvé

Plaza Imperial Tárraco. Vista antes de la urbanización | Vallvé

Escuela de Trabajo. Vista general | Teresa Andujar

Mas Potau. Entrada | Autor Desconocido

Proyecto para una nueva estación de ferrocarril en Tarragona | Autor Desconocido

Ferrocarril Tarragona-Reus. Nuevas infraestructuras | Autor Desconocido

Puerto. Proyecto de obras e instalaciones | Autor Desconocido

Tarragona. Proyecto del pantalán del río Francolí | Autor Desconocido

Fábrica de Harinas. Alzado principal | Autor Desconocido

Fábrica de Harinas. Planta | Autor Desconocido

Tarragona. Solares propiedad de D. Jacques Violet | Antonio Pujol

Tarragona. Vista aérea | Autor Desconocido

Tarragona. Vista aérea | Autor Desconocido

Casas Bajas de Torreforta. Vista general | Autor Desconocido

Proyecto de nueva estación de Tarragona. Fotomontaje | Vallvé

Tarragona. Cartel por paso de trenes Madrid-Barcelona | Vallvé

Puerto. Vista aérea | Autor Desconocido

Tabacalera. Plano general de la fábrica con el museo | Autor Desconocido Universidad Laboral. Vista de los terrenos | Autor Desconocido Casas Bajas de Torreforta. Entrega oficial de llaves | Chinchilla Universidad Laboral. Vista general en construcción | Valentí Canadell Plaza Imperial Tárraco. Obras de urbanización | Vallvé Plaza Imperial Tárraco. Obras de urbanización | Vallvé Tarragona. Barracas en entrevías de la playa del Miracle | Vallvé Tarragona. Barracas en entrevías de la playa del Miracle | Vallvé Tarragona. Vista antes de la construcción de la calle | Vallvé Universidad Laboral. Construcción de las aulas | Vallvé Plaza Imperial Tárraco. Vista aérea | Gaspar Ros

Universidad Laboral. Vista del complejo en construcción | Rafael Vidal Universidad Laboral. Vista del complejo en construcción | Rafael Vidal Universidad Laboral. Vista del comedor en construcción | Rafael Vidal Universidad Laboral. Vista del complejo en construcción | Rafael Vidal Universidad Laboral. Vista del comedor en construcción | Rafael Vidal Universidad Laboral. Vista del comedor en construcción | Rafael Vidal Universidad Laboral. Vista del comedor en construcción | Rafael Vidal Universidad Laboral. Vista del comedor en construcción | Rafael Vidal Universidad Laboral. Vista del comedor en construcción | Rafael Vidal Universidad Laboral. Vista del comedor en construcción | Rafael Vidal Universidad Laboral. Vista del comedor en construcción | Rafael Vidal La construcción de la Universidad Laboral de Tarragona | Gonzalo Peral La construcción de la Universidad Laboral de Tarragona | Gonzalo Peral La construcción de la Universidad Laboral de Tarragona | Gonzalo Peral La construcción de la Universidad Laboral de Tarragona | Gonzalo Peral La construcción de la Universidad Laboral de Tarragona | Gonzalo Peral Tarragona. Inauguración de la avenida del Conde de Vallellano | RTVE Tarragona. Vista aérea | Autor Desconocido

Torreforta. Plano de urbanización | Autor Desconocido Tarragona. Maqueta del auditorio del Campo de Marte | Vallvé
1955_APT_02

1956_AFT_01

1956_AFT_02

1956_AFT_03

1956_ATA_04

1956_ATA_05

1956_COACT_06

1956_COACT_07

1956_COACT_08

1956_COACT_09

1956_COACT_10

1956_ICC_11

1956_LAS_12

1956_NODO_13

1956_NODO_14

1956_TOT_15

1957_APT_01

1957_APT_02

1957_APT_03

1957_ATA_04

1957_EMT_05

1957_EMT_06

1957_LAS_07

1957_LAT_08

1957_LFU_09

1957_NODO_10

1957_TPT_11

1958_AAJMP_01

1958_AAJMP_02

1958_AFC_03

1958_APT_04

1958_LAH_05

1958_NODO_06

1958_ICC_07

1958_ICC_08

1959_AFC_01

1959_AFC_02

1959_AFC_03

1959_AFC_04

1959_AFC_05

1959_AFC_06

1959_AFC_07

1959_AFC_08

1959_AFC_09

1959_AFC_10

1959_AFC_11

1959_AFT_12

1959_AFT_13

1959_AFT_14

1959_AFT_15

1959_AFT_16

1959_AFT_17
Puerto. Vista aérea | Vallvé

Ferrocarril. Depósito de locomotoras en la zona de la playa del Miracle | Vallvé Tarragona. Vista aérea | Autor Desconocido

Universidad Laboral. Inauguración del complejo educativo | Vallvé

La Pineda. Vista aérea de Mas Potau | Autor Desconocido

Polígono Francolí. Vista aérea | Autor Desconocido

Universidad Laboral.Inauguración del complejo educativo | Gonzalo Peral Universidad Laboral.Inauguración del complejo educativo | Gonzalo Peral

Universidad Laboral.Inauguración del complejo educativo | Gonzalo Peral

Universidad Laboral.Inauguración del complejo educativo | Gonzalo Peral

Universidad Laboral.Inauguración del complejo educativo | Gonzalo Peral Litoral del Camp de Tarragona, Vuelo americano | ICC

Gobierno Civil. Dibujo del proyecto de Alejandro de la Sota | Alejandro de la Sota

Universidad Laboral. Inauguración del complejo educativo | RTVE

Tarragona. Electrificación del servicio ferroviario | RTVE

La Pineda. Vista aérea de Mas Potau | Autor Desconocido

Puerto. Vista aérea | Autor Desconocido

Puerto. Vista aérea | Autor Desconocido

Puerto. Vista general desde el mar | Autor Desconocido

Tarragona. Filovía | Joan Salvadó

Tarragona. Autobús ATSA | Archivo Fotográfico Canadell

Tarragona. Autobús ATSA | Archivo Fotográfico Canadell

Gobierno Civil. Vista del edificio en construcción | Autor Desconocido

Torreforta. Guardería del barrio | Chinchilla

Tarragona. Vista aérea | Autor Desconocido

Ciudad Residencial de Educación y Descanso. Visita del general Francisco| RTVE Universidad Laboral. Vista aérea de la plaza central | Autor Desconocido

Polígono Entrevías. Vista de una nave industrial | Arquitecto Pujol

Polígono Entrevías. Vista de una nave industrial | Arquitecto Pujol

Torreforta. Guardería del barrio | Valentí Canadell

Puerto. Vista aérea | Servicios Aéreos del Norte

Aeropuerto. Recepción del primer vuelo Madrid-Reus | Autor Desconocido Universidad Laboral. Vista de los talleres $\mid$ RTVE

El puerto de Tarragona y Entrevías | CETFA

Tarragona. Plano general | Enrique Kucera

Vista aérea de la estación de Clasificación | Valentí Canadell

Torreforta. Inauguración del colegio | Valentí Canadell

Camp de Tarragona | Valentí Canadell

Tarragona. Vista aérea de los polígonos industriales | Valentí Canadell

Tarragona. Vista aérea de los polígonos industriales | Valentí Canadel

Tarragona. Vista aérea de los polígonos industriales | Valentí Canadell

Tarragona. Vista aérea de los polígonos industriales | Valentí Canadell

Aliada Química S.A. Vista aérea | Valentí Canadell

La Canonja. Vista aérea | Valentí Canadell

Tabacalera. Vista aérea | Valentí Canadell

Tabacalera. Vista aérea | Valentí Canadell

Vista de los terrenos donde se construye el polígono Francolí | Vallvé

Torreforta. Calle en construcción | Vallvé

Ferrocarril. Vista de las vías de tren | Vallvé

Polígono Francolí. Vista de terrenos y primeras fábricas | Vallvé

Polígono Francolí. Vista de terrenos y primeras fábricas | Vallvé

Polígono Francolí. Vista de terrenos y primeras fábricas | Vallvé 
1959_AFT_18

1959_AFT_19

1959_AFT_20

1959_AFT_21

1959_ATA_22

1959_AFT_23

1959_LAT_24

1959_TPT_25

1960_AFC_01

1960_AFC_02

1960_AFC_03

1960_AFC_04

1960_AFC_05

1960_AFC_06

1960_AFC_07

1960_AFC_08

1960_AFC_09

1960_AFC_10

1960_AFC_11

1960_AFC_12

1960_AFC_13

1960_AFC_14

1960_AFC_15

1960_AFC_16

1960_AFC_17

1960_AFC_18

1960_AFT_19

1960_AFT_20

1960_ATA_21

1960_ATA_22

1960_ATA_23

1960_ATA_24

1960_COACT_25

1960_ICC_26

1960_ICC_27

1960_ICC_28

1960_ICC_29

1960 ICC 30

1960_ICC_31

1960_ICC_32

1960_PGO_33

1960_PGO_34

1960_PGO_35

1960_PGO_36

1960_PGO_37

1960_PGO_38

1960_PGO_39

1960_PGO_40

1960_PG0_41

1960_PGO_42

1960_PGO_43

1960_PGO_44
Polígono Francolí. Vista de terrenos y primeras fábricas | Vallvé Polígono Francolí. Vista de terrenos y primeras fábricas | Vallvé Polígono Francolí. Vista de terrenos y primeras fábricas | Vallvé Plaza Imperial Tárraco. Vista del puente | Chinchilla Tarragona. Vista aérea | Rafael Vidal Plaza Imperial Tárraco. Vista general | Autor Desconocido Torreforta. Entrega oficial viviendas de la plaza Primer de Maig | Chinchilla Universidad Laboral. Vista aérea | Autor Desconocido Campsa. Vista desde una masía próxima | Valentí Canadell Campsa. Vista desde una masía próxima | Valentí Canadell Campsa. Vista desde una masía próxima | Valentí Canadell Polígono Francolí. Vista de una masía | Valentí Canadell

Tarragona. Vista aérea | Valentí Canadell

Torreforta. Vista del colegio | Valentí Canadell

Torreforta. Vista de las viviendas | Valentí Canadell

Viviendas en construcción en Torreforta | Valentí Canadell

Viviendas en construcción en Torreforta | Valentí Canadell

Viviendas en construcción en Torreforta | Valentí Canadell

Viviendas en construcción en Torreforta | Valentí Canadell

Viviendas en construcción en Torreforta | Valentí Canadell

Torreforta. Vista de las viviendas | Valentí Canadell

Torreforta. Vista de las viviendas | Valentí Canadell

Torreforta. Vista de las viviendas | Valentí Canadell

La Canonja. Vista de una calle | Valentí Canadell

Cap de Salou | Valentí Canadell

La Pineda. Vista de la costa | Valentí Canadell

Ferrocarril. Vista de la estación y de las vías de tren | Vallvé

Ferrocarril. Depósito de locomotoras en la zona de la playa del Miracle | Vallvé

Siata. Feria de Valencia | Rafael Vidal

Rec Major | Magí Benito

Vista aérea de la avenida de Roma recién construida | Gaspar Ros

Rec Major | Rafael Vidal

Plaza Imperial Tárraco. Vista de la avenida de Roma | Arquitecto Monravà

Industria. Vista aérea | Servicios Aéreos Comerciales Españoles

Industria. Vista aérea | Servicios Aéreos Comerciales Españoles

Union Azufrera. Vista aérea | Servicios Aéreos Comerciales Españoles

Industria. Vista aérea | Servicios Aéreos Comerciales Españoles

Industria. Vista aérea | Servicios Aéreos Comerciales Españoles

Union Azufrera. Vista aérea | Servicios Aéreos Comerciales Españoles

Universidad Laboral. Vista aérea | Servicios Aéreos Comerciales Españoles

PGO. Topográfico | Arquitecto Monravà

PGO. Histórico artístico | Arquitecto Monravà

PGO. Alturas de edificación | Arquitecto Monravà

PGO. Vías principales y servicios | Arquitecto Monravà

PGO. Densidades | Arquitecto Monravà

PGO. Industrias y almacenes | Arquitecto Monravà

PGO. Comercio | Arquitecto Monravà

PGO. Alcantarillado | Arquitecto Monravà

PGO. Electricidad | Arquitecto Monravà

PGO. Servicio de Agua | Arquitecto Monravà

PGO. Servicio de Gas | Arquitecto Monravà

PGO. Plano viario | Arquitecto Monravà
1960_PGO_45

1960_PGO_46

1960_PG0_47

1960_PGO_48

1960_PG0_49

1960_PG0_50

1960_PG0_51

1960_PG0_52

1961_AAJMP_01

1961_AAJMP_02

1961_AAJMP_03

1962_ATA_01

1962_COACT_02

1962_COACT_03

1962_ICC_04

1962_ICC_05

1962 ICC 06

1962_ICC_07

1962_ICC_08

1962_ICC_09

1962_HLV_10

1962_IBM_11

1962_IBM_12

1962_IBM_13

1962_IBM_14

1962_IBM_15

1963_AAA_01

1963_AAJMM_02

1963_AAJMM_03

1963_AAJMM_04

1963_AAJMM_05

1963_AAJMM_06

1963_LITSC_07

1963_HABC_08

1963_HABC_09

1963_HLV_10

1963_HLV_11

1963_HLV_12

1963_HLV_13

1963_HLV_14

1963_HLV_15

1963_AAJMP_16

1963_AFC_17

1963_ICC_18

1963_NODO_19

1964_AFC_01

1964_ANC_02

1964_ATA_03

1964_ATA_04

1964_ATA_05

1964_ICC_06

1964_ICC_07
PGO. Plano Vías existentes y proyectadas | Arquitecto Monravà

PGO. Plano de zonificación | Arquitecto Monravà

PGO. Espacios libres | Arquitecto Monravà

PGO. Plano de organización | Arquitecto Monravà

PGO. Plan de etapas | Arquitecto Monravà

PGO. Perímetro exterior | Arquitecto Monravà

PGO. Servicios de agua y alcantarillado | Arquitecto Monravà

PGO. Relación de la ciudad con su comarca | Arquitecto Monravà

| Autor Desconocido

| Autor Desconocido

| Autor Desconocido

Ceratonia. Vista aérea | Félix Adserias

Vista del edificio principal de la fábrica Punta Bic|Autor Desconocido

Dibujo del proyecto para la fábrica Punta Bic de Laforest S.A. | J. Doménech

Fábrica Punta Bic. Vista aérea | Servicios Aéreos Comerciales Españoles

Puerto. Vista aérea | Servicios Aéreos Comerciales Españoles

Universidad Laboral. Vista aérea | Servicios Aéreos Comerciales Españoles

Universidad Laboral. Vista aérea | Servicios Aéreos Comerciales Españoles

Universidad Laboral. Vista aérea | Servicios Aéreos Comerciales Españoles

Universidad Laboral. Vista aérea | Servicios Aéreos Comerciales Españoles

"El Banco Mundial hace público su informe sobre la economía española”

Carta de presentación del informe del BIRF | Eugene B. Black

IBM. Plano distribución de estaciones de Clasificación | Autor Desconocido IBM. Plano ordenación de estaciones de Clasificación | Autor Desconocido IBM. Plano densidad del tráfico por carretera en I960 | Autor Desconocido IBM. Plano densidad de tráfico de la Renfe en I960 | Autor Desconocido

La Canonja. Avance del Plan General | Arquitecto Aragonés

Proyecto de almacén, estación de servicio y restaurante $\mid J$. M. Monravà

Proyecto de almacén, estación de servicio y restaurante | J. M. Monravà

Proyecto de almacén, estación de servicio y restaurante | J. M. Monravà

Proyecto de almacén, estación de servicio y restaurante | J. M. Monravà

Proyecto de almacén, estación de servicio y restaurante | J. M. Monravà

Vila-seca. Plan General | Archivo Municipal Vila-seca

Acerbi. Noticia publicitaria | Autor Desconocido

Aliada Química S.A. Inauguración de planta industrial | Autor Desconocido Universidad Laboral. Noticia "La verdad de nuestro tiempo" | T.A.F. Polígono Entrevías. Noticia “El alma nueva de Tarragona” | Horacio Saenz Tabacalera. Noticia “Tabacalera S.A.” | Autor Desconocido

Tarragona. Noticia “Tarragona de cara al futuro" | Autor Desconocido Salou. "En el horizonte mediterráneo de Tarragona” | Autor Desconocido Tarragona. Noticia “Hotel Imperial Tarraco" | Autor Desconocido

Frigoríficos de Tarragona S.A. Vista aérea | Arquitecto Pujol Ciudad Residencial de Educación y Descanso. Comedor | Valentí Canadell Universidad Laboral. Vista aérea | Servicios Aéreos Comerciales Españoles Llegada a Tarragona de la reliquia de San Pablo | RTVE Ciudad Residencial de Educación y Descanso. Vista general | Canadell La Fábrica de Tabacos y Campsa. Vista aérea | Autor Desconocido Abelló Oxígeno. Vista aérea | Autor Desconocido Salena S.A. Vista aérea | Adriano Cattarin

Plaza Imperial Tárraco. Vista área | Autor Desconocido Alena. Vista área | Servicios Aéreos Comerciales Españoles Aliada Química S.A. Vista aérea | Servicios Aéreos Comerciales Españoles 
1964_ICC_08 Aliada Química S.A. Vista aérea | Servicios Aéreos Comerciales Españoles

1964_ICC_09

1964_ICC_10

1964_ICC_11

1964_ICC_12

1964_ICC_13

1964_ICC_14

1964_ICC_15

1964_ICC_16

1964_ICC_17

1964_ICC_18

1964_LBT_19

1964_LBT_20

1964_HABC_21

1964_LAT_22

1964_LITSC_23

1964_LITSC_24

1965_AFC_01

1965_AFC_02

1965_AFC_03

1965_AFC_04

1965_AFC_05

1965_AFC_06

1965_AFC_07

1965_AFC_08

1965_AFC_09

1965_AFC_10

1965_AFC_11

1965_AFC_12

1965_AFC_13

1965_AFC_14

1965_AFC_15

1965_AFC_16

1965_AFC_17

1965_AFC_18

1965_AFC_19

1965_AFC_20

1965_AFC_21

1965_AFC_22

1965_AFC_23

1965_AFC_24

1965_AFC_25

1965_AFT_26

1965_ANC_27

1965_APT_28

1965_APT_29

1965_APT_30

1965_APT_31

1965_APT_32

1965_ICC_33

1965_ICC_34

1965_ICC_35

Cerámicas José Vilar. Vista aérea| Servicios Aéreos Comerciales Españoles Cía Ferguson. Vista aérea | Servicios Aéreos Comerciales Españoles

Cepsa. Vista aérea | Servicios Aéreos Comerciales Españoles

Fhinssa. Vista aérea| Servicios Aéreos Comerciales Españoles

Hidro-Nitro S.A. Vista aérea | Servicios Aéreos Comerciales Españoles

Hidro-Nitro S.A. Vista aérea | Servicios Aéreos Comerciales Españoles

Industria. Vista aérea | Servicios Aéreos Comerciales Españoles

Szidensticker. Vista aérea | Servicios Aéreos Comerciales Españoles

Talleres Sta. María. Vista área | Servicios Aéreos Comerciales Españoles

Unión Azufrera. Vista aérea | Servicios Aéreos Comerciales Españoles

Torreforta. Viviendas Casas Bajas | Vallvé

Torreforta. Bloques del Primer Plan Sindica | Vallvé

Tarragona. "Acontecimiento industrial en Tarragona" | Autor Desconocido

Torreforta. Plano de urbanización | Autor Desconocido

Plan de Ordenación de la Costa de la Provincia de Tarragona | A.H.T.

Industria. Propiedad de los terrenos $\mid$ Autor Desconocido

La Pineda. Vista aérea | Valentí Canadell

La Pineda. Vista aérea | Valentí Canadell

Butano S.A. Construcción de la factoría | Valentí Canadell

Butano S.A. Construcción de la factoría | Valentí Canadell

N-340. Accidente en la carretera | Valentí Canadell

N-340. Accidente en la carretera | Valentí Canadell

N-340. Accidente en la carretera | Valentí Canadell

N-340. Accidente en la carretera | Valentí Canadell

Universidad Laboral. Vista de las aulas | Valentí Canadell

Universidad Laboral. Vista de las aulas | Valentí Canadell

Polígono Entrevías. Campos de trigo de los alrededores | Valentí Canadell

Polígono Entrevías. Campos de trigo de los alrededores | Valentí Canadell

Secuencia desde Universidad Laboral a Tarragona | Valentí Canadell

Secuencia desde Universidad Laboral a Tarragona | Valentí Canadell

Secuencia desde Universidad Laboral a Tarragona | Valentí Canadell

Secuencia desde Universidad Laboral a Tarragona | Valentí Canadell

Secuencia desde Universidad Laboral a Tarragona | Valentí Canadell

Secuencia desde Universidad Laboral a Tarragona | Valentí Canadell

Secuencia desde Universidad Laboral a Tarragona | Valentí Canadell

Secuencia desde Universidad Laboral a Tarragona | Valentí Canadell

Secuencia desde Universidad Laboral a Tarragona | Valentí Canadell

Secuencia desde Universidad Laboral a Tarragona | Valentí Canadell

Secuencia desde Universidad Laboral a Tarragona | Valentí Canadell

Secuencia desde Universidad Laboral a Tarragona | Valentí Canadell

Secuencia desde Universidad Laboral a Tarragona | Valentí Canadell

Rambla Nova. Vista aérea| Autor Desconocido

Cepsa. Construcción muelle | Autor Desconocido

Puerto. Vista aérea| T.A.F.

Puerto. Vista aérea del muelle Transversal y Contradique | T.A.F.

Puerto. Vista aérea| T.A.F.

Puerto. Vista aérea| T.A.F.

Puerto. Vista aérea| T.A.F.

Cepsa. Vista aérea | Servicios Aéreos Comerciales Españoles

Cepsa. Vista aérea | Servicios Aéreos Comerciales Españoles

Hidrónimos. Vista área | Servicios Aéreos Comerciales Españoles
1965_ICC_36

1965_ICC_37

1965_ICC_38

1965_ICC_39

1965_ICC_40

1965_ICC_41

1965_ICC_42

1965_ICC_43

1965_ICC_44

1965_ICC_45

1965_ICC_46

1965_ICC_47

1965_ICC_48

1965_ICC_49

1965_LAT_50

1965_LAT_51

1965_LAT_52

1965_NOD0_53

1965_AAA_54

1965_AAA_55

1965_AAA_56

1965_AAA_57

1965_AAR_58

1965_AAR_59

1965_AAR_60

1965_TOC_61

1965_TOC_62

1965_TOC_63

1965_TOC_64

1965_PEOCPT_65 PEOCPT. Plano general | Autor Desconocido

1965_PEOCPT_66 PEOCPT. Plano general | Autor Desconocido

1965_PEOCPT_67 PEOCPT. Estructura industrial | Autor Desconocido

1965_PEOCPT_68 PEOCPT. Zona con preponderancia de arroz | Autor Desconocido

1965_PEOCPT_69 PEOCPT. Comunicaciones Puertos | Autor Desconocido

1965_PEOCPT_70 PEOCPT. Agua-Electricidad | Autor Desconocido

1965_PEOCPT_71 PEOCPT. Demografía-Evolución | Autor Desconocido

1965_PEOCPT_72 PEOCPT. Demografía-Población | Autor Desconocido

1965_PEOCPT_73 PEOCPT. Plano general | Autor Desconocido

1965_PEOCPT_74 PEOCPT. Plano de zonificación viviendas | Autor Desconocido

1966_AFC_01

1966_AFC_02

1966_AFC_03

1966_AFC_04

1966_AFC_05

1966_AFC_06

1966_AFC_07

1966_AFC_08

1966_AFC_09

1966_AFC_10

1966_AFT_11

1966_AFT_12

1966_APT_13
Seidenstiker. Vista de la fábrica | Valentí Canadell

Polígono Entrevías. Industria | Valentí Canadell

Polígono Entrevías. Industria | Valentí Canadell

Polígono Entrevías. Industria | Valentí Canadell

Butano S.A. Construcción de la factoría | Valentí Canadell

Butano S.A. Construcción de la factoría | Valentí Canadell

Butano S.A. Construcción de la factoría | Valentí Canadell

Butano S.A. Construcción de la factoría | Valentí Canadell

Butano S.A. Construcción de la factoría | Valentí Canadell

Butano S.A. Construcción de la factoría | Valentí Canadell

Disa. Vista aérea | Autor Desconocido

Asesa. Inauguración | Vallvé

Puerto. Vista del muelle transversal | T.A.F. 
1966_ANC_14 1966_ATA_15 1966_ICC_16 1966_ICC_17 1966_ICC_18 1966_ICC_19 1966_ICC_20 1966_ICC_21 1966_ICC_22 1966_ICC_23 1966_ICC_24 1966_ICC_25 1966_LBT_26 1966_LBT_27 1966_LGF_28 1966_AAAP_29 1966_AAAP_30 1966_AAAP_31 1966_AAAP_32 1966_AAAP_33 1967_AFC_01 1967_AFC_02 1967_AFC_03 1967_AFT_04 1967_AFT_05 1967_AFT_06 1967_AFT_07 1967_AFT_08 1967_AFT_09 1967_AFT_10 1967_AFT_11 1967_AFT_12 1967_AFT_13 1967_AFT_14 1967_AFT_15 1967_AFT_16 1967_AFT_17 1967_AFT_18 1967_AFT_19 1967_AFT_20 1967_AFT_21 1967_AFT_22 1967_APT_23 1967_ATA_24 1967_EMT_25 1967_ICC_26 1967_ICC_27 1967_ICC_28 1967_ICC_29 1967_ICC_30 1967_ICC_31 1967_ICC_32
Disa. Vista panorámica | Autor Desconocido

Fábrica Seidensticker. Foto del conjunto de empleadas | Autor Desconocido Cía Ferguson. Vista aérea | Servicios Aéreos Comerciales Españoles Cía Ferguson. Vista aérea | Servicios Aéreos Comerciales Españoles Industrias Químicas Asociadas. Vista | Servicios Aéreos Comerciales Españoles Industrias Químicas Asociadas. Vista | Servicios Aéreos Comerciales Españoles Industrias Químicas Asociadas. Vista | Servicios Aéreos Comerciales Españoles Industrias Químicas Asociadas. Vista | Servicios Aéreos Comerciales Españoles Industria. Vista aérea | Servicios Aéreos Comerciales Españoles Industria. Vista aérea | Servicios Aéreos Comerciales Españoles Unión Azufrera. Vista aérea | Servicios Aéreos Comerciales Españoles Polígono Entrevías. Vista aérea | Servicios Aéreos Comerciales Españoles Bonavista. Autoconstrucción de una casa | Autor Desconocido Bonavista. Calle veintiuno | Olivé-Abelló Puerto. Vista aérea | Autor Desconocido Proyecto de desarrollo residencial en La Pineda. Sección | Alain Peron Alternativa para la ordenación de La Pineda | Alain Peron Alternativa para la ordenación de La Pineda | Alain Peron Alternativa para la ordenación de La Pineda | Alain Peron Alternativa para la ordenación de La Pineda | Alain Peron Industria. Vista de una nave | Valentí Canadell Antiguas instalaciones de Siata | Valentí Canadell Siata. Vista del polígono industrial Entrevías | Valentí Canadell Tarragona. Barracas bajo el puente del Francolí| Vallvé Tarragona. Barracas bajo el puente del Francolí| Vallvé Tarragona. Barracas bajo el puente del Francolí | Vallvé Tarragona. Barracas bajo el puente del Francolí | Vallvé Tarragona. Barracas bajo el puente del Francolí | Vallvé Tarragona. Barracas bajo el puente del Francolí | Vallvé Tarragona. Barracas bajo el puente del Francolí| Vallvé Alquímia. Barracas al lado de la empresa | Vallvé Alquímia. Barracas al lado de la empresa | Vallvé Alquímia. Barracas al lado de la empresa | Vallvé Alquímia. Barracas al lado de la empresa | Vallvé Alquímia. Barracas al lado de la empresa | Vallvé Polígono Entrevías. Barracas en los terrenos | Vallvé Polígono Entrevías. Barracas en los terrenos | Vallvé Polígono Entrevías. Barracas en los terrenos | Vallvé Polígono Entrevías. Barracas en los terrenos | Vallvé Polígono Entrevías. Barracas en los terrenos | Vallvé Polígono Entrevías. Barracas en los terrenos | Vallvé Polígono Entrevías. Barracas en los terrenos | Vallvé Puerto. Vista aérea | Autor Desconocido Seidenstiker. Vista de la fábrica | Rafael Vidal Tarragona. Accidente de autobús en el río Francolí | Autor Desconocido Butano S.A. y Punta Bic. Vista aérea de sus primeras instalaciones | SACE Icsasa. Vista aérea | Servicios Aéreos Comerciales Españoles Icsasa. Vista aérea | Servicios Aéreos Comerciales Españoles Icsasa y Cepsa. Vista aérea | Servicios Aéreos Comerciales Españoles Icsasa y Cepsa. Vista aérea | Servicios Aéreos Comerciales Españoles Icsasa y Cepsa. Vista aérea | Servicios Aéreos Comerciales Españoles Icsasa y Cepsa. Vista aérea | Servicios Aéreos Comerciales Españoles
1967_ICC_33

1967_ICC_34

1967_ICC_35

1967 HLV_36

1968_AFT_01

1968_AFT_02

1968_AFT_03

1968_AIA_04

1968_APT_05

1968_ICC_06

1968_ICC_07

1968_ICC_08

1968_ICC_09

1968_LBT_10

1968_LITSC_11

1968_LITSC_12

1968_LITSC_13

1968_LIT_14

1968_LIT_15

1969_AAR_01

1969_AAR_02

1969_AFT_03

1969_AFT_04

1969_AFT_05

1969_AFT_06

1969_AFT_07

1969_AFT_08

1969_AFT_09

1969_ICC_10

1969_ICC_11

1969_ICC_12

1969_ICC_13

1969_ICC_14

1969_ICC_15

1969_LITSC_16

1969_HLV_17

1969_HLV_18

1969_AAR_19

1969_AAR_20

1969_LITSC_21

1970_AFC_01

1970_AFC_02

1970_AFC_03

1970_AFC_04

1970_AFC_05

1970_AFC_06

1970_AFC_07

1970_AFC_08

1970_AFC_09

1970_AFC_10

1970_AFC_11

1970_AFC_12
Abelló Oxígeno. Vista aérea | Servicios Aéreos Comerciales Españoles Tarragona. Fotoplano | Servicios Aéreos Comerciales Españoles Tarragona. Fotoplano | Servicios Aéreos Comerciales Españoles Tarragona. Noticia "Fábricas en el paisaje” | Álvaro Ruibal IV Congreso Nacional de Arquitectura Típica Regional | Vallvé IV Congreso Nacional de Arquitectura Típica Regional | Vallvé Refinería. Rueda de prensa en Madrid | Sancho

Vila-seca. Vista de las "cuatro carreteras" y el Carrilet | Autor Desconocido Tarragona. Vista aérea $\mid S A E$

N-340 a su paso por La Canonja | Servicios Aéreos Comerciales Españoles Basf. Vista aérea | Servicios Aéreos Comerciales Españoles

Polígono Entrevías. Vista aérea | Servicios Aéreos Comerciales Españoles Polígono Entrevías. Vista aérea Servicios Aéreos Comerciales Españoles Bonavista. Familia junto a viviendas autoconstruidas | Autor Desconocido Dusa. Vista aérea | Suplemento regional

Industrias Químicas Asociadas. Vista de la fábrica | Bartomeu Esteve Bofarull Polígono Entrevías. Vista aérea | Geografia de Catalunya III

Plan Ordenación Comarcal. Núcleo central zona trébol | Autor Desconocido Plan Ordenación Comarcal. Polígonos industriales | Autor Desconocido

La Granja. Edificio de viviendas | Autor Desconocido

La Granja. Edificio de viviendas | Autor Desconocido

Refinería. Presentación en Madrid | Sancho

Refinería. Presentación en Madrid | Sancho

Refinería. Presentación en Madrid | Sancho

Refinería. Manifestación en agradecimiento a la industria | Vallvé

Sant Salvador. Barracones del grupo escolar del barrio | Vallvé

Basf. Inauguración de la fábrica | Vallvé

Basf. Vista aérea | Autor Desconocido

Cepsa. Vista aérea | Servicios Aéreos Comerciales Españoles

Industrias Químicas Asociadas. Vista | Servicios Aéreos Comerciales Españoles Industrias Químicas Asociadas. Vista | Servicios Aéreos Comerciales Españoles Unión Azufrera. Vista aérea| Servicios Aéreos Comerciales Españoles Unión Azufrera. Vista aérea | Servicios Aéreos Comerciales Españoles Unión Azufrera. Vista aérea | Servicios Aéreos Comerciales Españoles Basf. Vista de la factoría en construcción | Bartomeu Esteve Bofarull Basf. Noticia de inauguración | Autor Desconocido

Tarragona. Noticia "Sólido proceso de industrialización” | Mezquida

La Granja. Plano edificio de viviendas | Arquitecto Ripoll

La Granja. Plano edificio de viviendas | Arquitecto Ripoll Industria. Plano de la situación de los terrenos $\mid$ Autor Desconocido Industria. Vista de una nave | Valentí Canadell Industria. Vista de una nave | Valentí Canadell Tarragona. Vista del puente sobre el Francolí| Valentí Canadell Tarragona. Vista del río Francolí| Valentí Canadell Tarragona. Barracas bajo el puente del Francolí | Valentí Canadell Tarragona. Barracas bajo el puente del Francolí | Valentí Canadell Tarragona. Barracas bajo el puente del Francolí | Valentí Canadell Tarragona. Barracas bajo el puente del Francolí | Valentí Canadell Tarragona. Barracas bajo el puente del Francolí| Valentí Canadell Tarragona. Barracas junto al río Francolí | Valentí Canadell Tarragona. Barracas junto al río Francolí | Valentí Canadell Tarragona. Barracas junto al río Francolí | Valentí Canadell 
1970_AFC_13 Tarragona. Barracas junto al río Francolí | Valentí Canadell

1970_AFC_14 Tarragona. Barracas junto al río Francolí, al fondo, Campsa | Valentí Canadell

1970_AFC_15 Tarragona. Barracas junto al río Francolí | Valentí Canadell

1970_AFC_16 Tarragona. Barracas junto al río Francolí | Valentí Canadell

1970_AFF_17 Tarragona. Vista autobús Firtesa | Autor Desconocido

1970_AFF_18 Tarragona. Vista autobús Firtesa | Autor Desconocido

1970_AFG_19 La Granja. Edificio de viviendas | Autor Desconocido

1970_AFG_20 La Granja. Calle de Gran Canaria | Autor Desconocido

1970_AFG_21

1970_AFT_22

1970_AFT_23

1970_APT_24

1970_ATA_25

1970_ATA_26

1970_ICC_27

1970_ICC_28

1970_ICC_29

1970_ICC_30

1970_ICC_31

1970_LBS_32

1970_LBT_33

1970_LBT_34

1970_LBT_35

1970_LBT_36

1970_LBT_37

1970_HLV_38

1970_PCTRV_39

1970_PCTRV_40

1970_PCTRV_41

1970_PCTRV_42

1970_PCTRV_43

1970_PCTRV_44

1970_PCTRV_45

1970_PCTRV_46

1970_PCTRV_47

1970_PCTRV_48

1970_PCTRV_49

1970_PCTRV_50

1970 PCTRV 51

1970_PCTRV_52

1970_PCTRV_53

1970_PCTRV_54

1971_APT_01

1971_APT_02

1971_APT_03

1971_ATA_04

1971_ICC_05

1971_ICC_06

1971_ICC_07

1971_LGF_08

1971_NODO_09

1971_NODO_10

La Granja. Colegio de La Salle | Autor Desconocido

Desbordamiento en la desembocadura del río Francolí| Chinchilla Desbordamiento en la desembocadura del río Francolí | Chinchilla

Puerto. Vista aérea de la dársena interior | Raymond

Desbordamiento en la desembocadura del río Francolí| Chinchilla

Desbordamiento en la desembocadura del río Francolí | Chinchilla

Basf. Vista aérea | Servicios Aéreos Comerciales Españoles

Basf. Vista aérea | Servicios Aéreos Comerciales Españoles

Bic. Vista aérea | Servicios Aéreos Comerciales Españoles

Dow Unquinesa. Vista aérea | Servicios Aéreos Comerciales Españoles

Dow Unquinesa. Vista aérea | Servicios Aéreos Comerciales Españoles

Bonavista. Escolares en el patio-calle | Vallvé

Industria. Complejo petroquímico de la Zona Trébol | Federación AAVV

Torreforta. Vista de una calle | Vallvé

Torreforta. Vista de la parroquia | Olivé-Abelló

El Pilar. Vista del barrio | Olivé-Abelló

Bonavista. Vista de las viviendas de Parcelas Tuset | Autor Desconocido

Basf. Noticia publicitaria | Autor Desconocido

PCTRV. Área del Plan Comarcal | Manuel Cortes

PCTRV. Zonas geográficas y límites | Manuel Cortes

PCTRV. Mapa geológico del área | Manuel Cortes

PCTRV. Densidad de población | Manuel Cortes

PCTRV. Distribución de habitantes en núcleos población | Manuel Cortes

PCTRV. Distribución agraria de la superficie | Manuel Cortes

PCTRV. Localización industrial | Manuel Cortes

PCTRV. Dependencia municipios de núcleos comerciales | Manuel Cortes

PCTRV. Carreteras | Manuel Cortes

PCTRV. Distribución tráfico carretera aforado Tarragona | Manuel Cortes

PCTRV. Distribución tráfico carretera aforado Reus | Manuel Cortes

PCTRV. Transporte público de viajeros por carretera | Manuel Cortes

PCTRV. Intensidad de tráfico de la red ferroviaria | Manuel Cortes

PCTRV. Monumentos y playas | Manuel Cortes

PCTRV. Líneas eléctricas | Manuel Cortes

PCTRV. Área de revisión del Plan General y Comarcal | Manuel Cortes

Puerto. Vista aérea de los diques de Levante y de Aragón | T.A.F.

Puerto. Vista aérea de las obras de los diques de Levante y de Aragón | T.A.F.

Puerto. Vista aérea de las obras del dique de Levante | T.A.F.

Tarragona. Maqueta del edificio de La Caixa | Ramón Fraxedas

Tarragona. Fotoplano | Servicios Aéreos Comerciales Españoles

Tarragona. Fotoplano | Servicios Aéreos Comerciales Españoles

Tarragona. Fotoplano | Servicios Aéreos Comerciales Españoles

Puerto. Vista general | Autor Desconocido

Industria. Petróleo en Sant Carles de la Ràpita | RTVE

Puerto. Grúas y diques de Tarragona | RTVE
1971_LIT_11

1971_LIT_12

1971_LITSC_13

1972_AAA_01

1972_AAA_02

1972_AAMA_03

1972_AFC_04

1972_AFG_05

1972_APT_06

1972_APT_07

1972_APT_08

1972_ICC_09

1972_ICC_10

1972_ICC_11

1972_ICC_12

1972_LBT_13

1972_TPT_14

1972_HLV_15

1972_HLV_16

1972_PCTRV_17

1972_PCTRV_18

1972_PCTRV_19

1972_PCTRV_20

1972_PCTRV_21

1972_PCTRV_22

1973_COACT_01

1973_COACT_02

1973_COACT_03

1973_ICC_04

1973_ICC_05

1973_ICC_06

1973_ICC_07

1973_ICC_08

1973_ICC_09

1973_ICC_10

1973_ICC_11

1973_LUL_12

1973_LUL_13

1973_RPGO_14

1973_RPGO_15

1973_RPG0_16

1973_RPGO_17

1973_RPG0_18

1973_RPG0_19

1973_RPG0_20

1973_RPGO_21

1973_RPGO_22

1973_RPGO_23

1973_RPGO_24

1973_RPGO_25

1973_RPGO_26

1973_RPGO_27
PCTRV. Refinería en la provincia de Tarragona | Autor Desconocido PCTRV. Polígono de La Canonja | Autor Desconocido

PCTRV. Zonificación | Archivo Histórico Diputación Tarragona

Bonavista. Vista aérea | Arquitecto Aragonés

Polígono Entrevías. Vista aérea | Arquitecto Aragonés

Construcción del viaducto de la autopista sobre el Francolí | Autor Desconocido

Basf. Vista de la fábrica | Valentí Canadell

La Granja. Calle de Gran Canaria | Autor Desconocido

Puerto. Vista aérea del nuevo muelle de Aragón | T.A.F.

Puerto. Vista aérea de las obras del dique de Levante | T.A.F.

Puerto. Vista aérea del muelle de Aragón | T.A.F.

Industria. Vista aérea | Servicios Aéreos Comerciales Españoles

Industria. Vista aérea | Servicios Aéreos Comerciales Españoles

Industria. Vista aérea | Servicios Aéreos Comerciales Españoles

Industria. Vista aérea | Servicios Aéreos Comerciales Españoles

Bonavista. Lugar de reunión habitual de los jubilados | Autor Desconocido

Universidad Laboral. Vista aérea | Autor Desconocido

Unión Explosivos Río Tinto. Noticia | Autor Desconocido

Basf. Noticia publicitaria | Autor Desconocido

PCTRV. Zonas urbanizadas | Manuel Cortes

PCTRV. Zonificación | Manuel Cortes

PCTRV. Zonificación | Manuel Cortes

PCTRV. Zonificación | Manuel Cortes

PCTRV. Zonificación | Manuel Cortes

PCTRV. Red viaria | Manuel Cortes

Universidad Laboral. Vista aérea | Autor Desconocido

Universidad Laboral. Vista aérea | Autor Desconocido

Universidad Laboral. Vista aérea | Autor Desconocido

Dow Unquinesa. Vista aérea | Servicios Aéreos Comerciales Españoles

Dow Unquinesa. Vista aérea | Servicios Aéreos Comerciales Españoles

Dow Unquinesa. Vista aérea | Servicios Aéreos Comerciales Españoles

Dow Unquinesa. Vista aérea | Servicios Aéreos Comerciales Españoles

Dow Unquinesa. Vista aérea | Servicios Aéreos Comerciales Españoles

Dow Unquinesa. Vista aérea | Servicios Aéreos Comerciales Españoles

Dow Unquinesa. Vista aérea | Servicios Aéreos Comerciales Españoles

Dow Unquinesa. Vista aérea | Servicios Aéreos Comerciales Españoles

Universidad Laboral. Vista aérea | Autor Desconocido

Universidad Laboral. Vista aérea | Autor Desconocido

RPGO. Información planos | Francisco Moreno / Manuel Cortes

RPGO. Aplicación nuevas normas | Francisco Moreno / Manuel Cortes

RPGO. Viales | Francisco Moreno / Manuel Cortes

RPGO. Zonificación | Francisco Moreno / Manuel Cortes

RPGO. Viales | Francisco Moreno / Manuel Cortes

RPGO. Viales | Francisco Moreno / Manuel Cortes

RPGO. Viales | Francisco Moreno / Manuel Cortes

RPGO. Viales | Francisco Moreno / Manuel Cortes

RPGO. Viales | Francisco Moreno / Manuel Cortes

RPGO. Viales | Francisco Moreno / Manuel Cortes

RPGO. Viales | Francisco Moreno / Manuel Cortes

RPGO. Zonificación | Francisco Moreno / Manuel Cortes

RPGO. Viales | Francisco Moreno / Manuel Cortes

RPGO. Viales | Francisco Moreno / Manuel Cortes 
1973_AAPM_28

1973_STUT_29

1974_APT_01

1974_APT_02

1974_APT_03

1974_APT_04

1974_ICC_05

1974_ICC_06

1974_HLV_07

1974_AAPM_08

1974_AAPM_09

1974_AAPM_10

1974_AAPM_11

1974_AAPM_12

1974_COACT_13

1975_APT_01

1975_APT_02

1975_APT_03

1975_APT_04

1975_HABC_05

1975_HLV_06

1976_APT_01

1976_APT_02

1976_APT_03

1976_ATA_04

1976_EMT_05

1976_EMT_06

1976_EMT_07

1976_EMT_08

1976_LBT_09

1976_LBT_10

1976_LBT_11

1977_AFG_01

1977_APT_02

1977_APT_03

1977_APT_04

1977_ICC_05

1977_ICC_06

1977_ICC_07

1977_ICC_08

1977_ICC_09

1977_LBT_10

1978_LGF_01

1979_ICC_01

1979_ICC_02

1979_LBT_03

1979_LBT_04

1979_LBT_05

1979_PGO_06

1979_PGO_07

1979_PGO_08

1979_PGO_09
Tarragona. Plano general | Pascual Monravà

Tarragona. Mapa topográfico | Instituto Geográfico Nacional

Asesa. Vista aérea | T.A.F.

Asesa y Enpetrol. Vista aérea| T.A.F.

Industria. Vista aérea refinería y estribo pantalán | Paisajes Españoles FAEI

Asesa. Vista aérea del puerto y del pantalán | T.A.F.

El Puerto. Vista aérea | Servicios Aéreos Comerciales Españoles

El Puerto. Vista aérea | Servicios Aéreos Comerciales Españoles

Alcudia S.A. Noticia publicitaria | Autor Desconocido

Cinturón (2. ${ }^{a}$ fase). Plano del conjunto | Pascual Monravà

Cinturón (2. ${ }^{a}$ fase). Planta y perfil longitudinal | Pascual Monravà

Cinturón (2. ${ }^{a}$ fase). Planta y perfil longitudinal | Pascual Monravà

Cinturón (2. ${ }^{a}$ fase). Planta y perfil longitudinal | Pascual Monravà

Cinturón (2. ${ }^{a}$ fase). Plano índice | Pascual Monravà

Tabacalera. Plano de curvas de nivel | Autor Desconocido

Puerto. Vista aérea | Autor Desconocido

Puerto. Vista aérea | Paisajes Españoles FAEI

Puerto. Vista aérea | T.A.F.

Puerto. Vista aérea | T.A.F.

Bayer. Noticia publicitaria | Autor Desconocido

Expoquímica 75. Noticia publicitaria | Gene Gregory

Puerto. Vista aérea de nuevos proyectos de ampliación | Autor Desconocido

Puerto. Obras del muelle de Aragón | Paisajes Españoles FAEI

Puerto. Vista aérea | T.A.F.

Barrio de La Esperanza. Vista de las viviendas | Autor Desconocido

Tarragona. Autobús articulado Gasol | Miguel Llevat

Tarragona. Autobús articulado Gasol | Miguel Llevat

Tarragona. Autobús Gasol | Miguel Llevat

Tarragona. Autobús Gasol | Miguel Llevat

La Esperanza. Inauguración oficial del barrio | Chinchilla

Icomar. Vista de la fachada de la urbanización | Autor Desconocido

Bonavista. Concentración por un Ambulatorio | Federación AAVV

La Granja. Construcción de las viviendas | Autor Desconocido

Puerto. Vista aérea de los muelles Aragón, Lérida y Castilla | Autor Desconocido

Puerto. Vista aérea del dique de Levante | Paisajes Españoles FAEI

Asesa y Enpetrol. Vista aérea | T.A.F.

Tarragona. Fotoplano | Servicios Aéreos Comerciales Españoles

Tarragona. Fotoplano | Servicios Aéreos Comerciales Españoles

Tarragona. Fotoplano | Servicios Aéreos Comerciales Españoles

Tarragona. Fotoplano | Servicios Aéreos Comerciales Españoles

Tarragona. Fotoplano | Servicios Aéreos Comerciales Españoles

La Granja. Vista de la avenida central | Olivé-Abelló

Puerto. Vista aérea | Autor Desconocido

Tarragona. Puentes ferroviarios | Servicios Aéreos Comerciales Españoles

Puerto. Vista aérea | Servicios Aéreos Comerciales Españoles

Riuclar. Inundaciones "Pequeña Venecia” | A.V.V. Riuclar

Riuclar. Manifestaciones por colegio digno | Agramunt Diario de Tarragona

Campclar. Bloques de promoción pública | Olivé-Abelló

PGO. Plano de La Canonja | Autor Desconocido

PGO. Bonavista | Autor Desconocido

PGO. La Granja, Torreforta, Riuclar | Autor Desconocido

PGO. Zona Educacional | Autor Desconocido
1979_PGO_10

1979_PGO_11

1979_PG0_12

1979_PG0_13

1980_CMC_01

1980_COACT_02

1980_HLV_03

1980_HLV_04

1981_STUT_01

1983_COACT_01

1983_COACT_H-20-12

1983_COACT_H-20-13

1983_COACT_H-20-14

1983_COACT_H-20-15

1983_COACT_H-20-16

1983_COACT_H-21-12

1983_COACT_H-21-13

1983_COACT_H-21-14

1983_COACT_H-21-15

1983_COACT_H-21-16

1983_COACT_H-21-17

1983_COACT_H-21-18

1983_COACT_H-21-19

1983_COACT_H-22-12

1983_COACT_H-22-13

1983_COACT_H-22-14

1983_COACT_H-22-15

1983_COACT_H-22-16

1983_COACT_H-22-17

1983_COACT_H-22-18

1983_COACT_H-22-19

1983_COACT_H-23-11

1983_COACT_H-23-12

1983_COACT_H-23-13

1983_COACT_H-23-14

1983_COACT_H-23-15

1983_COACT_H-23-16

1983_COACT_H-23-17

1983_COACT_H-23-18

1983_COACT_H-23-19

1983_COACT_H-24-11

1983_COACT_H-24-12

1983_COACT_H-24-13

1983_COACT_H-24-14

1983_COACT_H-24-15

1983 COACT H-24-16

1983_COACT_H-24-17

1983 COACT H-24-18

1983_COACT_H-24-19

1983_COACT_H-25-11

1983_COACT_H-25-12

1983_COACT_H-25-13
GO. Campclar | Autor Desconocido

G. Campclar | Autor Desconocido

. Distribución de los Habitantes | Autor Desconocido

O. Edificación | Autor Desconocido

no de propuestas e iniciativas | Autor Desconocido

ragona. Plano general | Otecar

ustria. Noticia del Plan Parcial de Gran Industria | Félix Llovell dustria. Aprobado Plan Parcial de Gran Industria | Félix Llovell

rgona. Cartografía militar | Servicio Geográfico del Ejército

ona. Plano general | Otecar

Tarragona. Plano Hoja 2O-I2 | Otecar

Tarragona. Plano Hoja 20-I3 | Otecar

Tarragona. Plano Hoja 20-I4 | Otecar

Tarragona. Plano Hoja 20-I5 $\mid$ Otecar

Tarragona. Plano Hoja 20-I6 | Otecar

Tarragona. Plano Hoja 2I-I2 | Otecar

Tarragona. Plano Hoja 2I-I3 | Otecar

Tarragona. Plano Hoja 2I-I4 | Otecar

Tarragona. Plano Hoja 2I-I5 | Otecar

Tarragona. Plano Hoja 2I-I6 | Otecar

Tarragona. Plano Hoja 2I-I7 | Otecar

Tarragona. Plano Hoja 2I-I8 | Otecar

Tarragona. Plano Hoja 2I-I9 | Otecar

Tarragona. Plano Hoja 22-I2 | Otecar

Tarragona. Plano Hoja 22-13 | Otecar

Tarragona. Plano Hoja 22-I4 | Otecar

Tarragona. Plano Hoja 22-15 | Otecar

Tarragona. Plano Hoja 22-I6 | Otecar

Tarragona. Plano Hoja 22-I7 | Otecar

Tarragona. Plano Hoja 22-I8 | Otecar

Tarragona. Plano Hoja 22-I9 | Otecar

Tarragona. Plano Hoja 23-II | Otecar

Tarragona. Plano Hoja 23-I2 | Otecar

Tarragona. Plano Hoja 23-I3 | Otecar

Tarragona. Plano Hoja 23-I4 | Otecar

Tarragona. Plano Hoja 23-I5 | Otecar

Tarragona. Plano Hoja 23-I6 | Otecar

Tarragona. Plano Hoja 23-I7 | Otecar

Tarragona. Plano Hoja 23-I8 | Otecar

Tarragona. Plano Hoja 23-I9 | Otecar

Tarragona. Plano Hoja 24-II | Otecar

Tarragona. Plano Hoja 24-I2 | Otecar

Tarragona. Plano Hoja 24-I3 | Otecar

Tarragona. Plano Hoja 24-I4 | Otecar

Tarragona. Plano Hoja 24-15 | Otecar

Tarragona. Plano Hoja 24-I6 | Otecar

Tarragona. Plano Hoja 24-I7 | Otecar

Tarragona. Plano Hoja 24-I8 | Otecar

Tarragona. Plano Hoja 24-I9 | Otecar

Tarragona. Plano Hoja 25-II | Otecar

Tarragona. Plano Hoja 25-I2 | Otecar

Tarragona. Plano Hoja 25-13 | Otecar 
1983_COACT_H-25-14 Tarragona. Plano Hoja 25-I4 | Otecar

1983_COACT_H-25-15 Tarragona. Plano Hoja 25-I5 | Otecar

1983_COACT_H-25-16 Tarragona. Plano Hoja 25-16 | Otecar

1983_COACT_H-26-11 Tarragona. Plano Hoja 26-II | Otecar

1983_COACT_H-26-12 Tarragona. Plano Hoja 26-I2 | Otecar

1983_COACT_H-26-13 Tarragona. Plano Hoja 26-13 | Otecar

1983_COACT_H-26-14 Tarragona. Plano Hoja 26-I4 | Otecar

1983_COACT_H-26-15 Tarragona. Plano Hoja 26-15 | Otecar

1983_COACT_H-27-11 Tarragona. Plano Hoja 27-II | Otecar

1983_COACT_H-27-12 Tarragona. Plano Hoja 27-I2 | Otecar

1983_COACT_H-27-13 Tarragona. Plano Hoja 27-I3 | Otecar

1983_COACT_H-27-14 Tarragona. Plano Hoja 27-I4 | Otecar

1983_COACT_H-27-15 Tarragona. Plano Hoja 27-I5 | Otecar

1983_COACT_H-28-14 Tarragona. Plano Hoja 28-I4 | Otecar

\section{3_ICC_58 Tarragona. Ortofoto | ICC}

1983_PGOUT_59 PGOUT. Plano de zonificación urbanística | Lluís Cantallops y Valeri

1983_PGOUT_60 PGOUT. Propuesta esquemática del Plan | Lluís Cantallops Valeri

1983_PGOUT_61 PGOUT. Estructura general del Plan | Lluís Cantallops y Valeri

1983_PGOUT_62 PGOUT. Morfología urbana | Lluís Cantallops y Valeri

1984_LBT_01_Riuclar. Vista general | Archivo Municipal

1985_AFT_01 Tarragona. Vista aérea | Autor Desconocido

1985_HDT_02 N-340. Vista aérea | Autor Desconocido

1985_LAR_03

1985_LAR_04

1985_LGC_05

1985_LLC_06

1985_LBT_07

1985_LBT_08

1985_LBT_09

1985_LBT_10

1985_STUT_11

1986_ICC_01

1986_ICC_02

1986_ICC_03

1986_ICC_04

1986_ICC_05

1986_ICC_06

1986 ICC 07

1986_ICC_08

1986_ICC_09

1987_APT_10

1987_ICC_11

1987_STUT_12

1987_STUT_13

1988_STUT_01

1989_LAC_01

1990_APT_01

1990_APT_02

1990_APT_03

1990_LAC_04

1990_LAH_05

1990_LAR_06
Torreforta e Icomar. Vista aérea | Autor Desconocido

Universidad Laboral. Vista aérea | Autor Desconocido

La Canonja. Vista del desaparecido bajador de tren | Autor Desconocido

Bonavista. Plano general | Autor Desconocido

Campclar. Plano general | Autor Desconocido

Torreforta. Plano general | Autor Desconocido

Riuclar e Icomar. Plano general | Autor Desconocido

Tarragona. Plano general | Trabajos Catastrales

Industria y puerto. Vista aérea | Servicios Aéreos Comerciales Españoles

Industria y puerto. Vista aérea | Servicios Aéreos Comerciales Españoles

Industria y puerto. Vista aérea | Servicios Aéreos Comerciales Españoles

Industria y puerto. Vista aérea | Servicios Aéreos Comerciales Españoles

Industria y puerto. Vista aérea | Servicios Aéreos Comerciales Españoles

Industria y puerto. Vista aérea | Servicios Aéreos Comerciales Españoles

Industria y puerto. Vista aérea | Servicios Aéreos Comerciales Españoles

Industria y puerto. Vista aérea | Servicios Aéreos Comerciales Españoles

Industria y puerto. Vista aérea | Servicios Aéreos Comerciales Españoles

Puerto. Vista aérea | Chinchilla

Tarragona. Plano general | Generalitat de Catalunya

Tarragona. Plano red de caminos | Diputación de Tarragona

Tabacalera, Campsa y el río Francolí | ICC

Bloques de viviendas y terrenos en Campclar | Autor Desconocido

Eje transversal de acceso al puerto. Vista aérea | Paisajes Españoles FAEI

Polígono Francolí. Vista aérea | J. Farré

Puerto. Vista aérea | Altair Photo S.A.

N-340 a su paso por Campclar en dirección a Tarragona | Autor Desconocido

Aeropuerto. Vista de la pista | Autor Desconocido

Riuclar. Vista del cauce del río Clar seco | Autor Desconocido
Riuclar. Alrededores del barrio | Autor Desconocido

Cataluña. Imagen de satélite $\mid$ ICC
1990_LAR_07

1990_LAR_08

1990_LAR_09

1990_LAR_10

1991_APT_01

1992_APT_02

1994_AFT_01

1994_APT_02

1994_APT_03

1994_ATA_04

1994_ATA_05

1994_ATA_06

1996_ICC_01

1997_HLV_01

1999_HLV_01

2000_AFA_01

2000_AFA_02

2000_AFA_03

2000_AFA_04

2000_AFA_05

2000_AFA_06

2000_AFA_07

2000_AFA_08

2000_AFA_09

2000_AFA_10

2000_AFA_11

2000_AFA_12

2000_AFA_13

2000_AFA_14

2000_APT_15

2000_APT_16

2000_APT_17

2000_LLC_18

2000_LLC_19

2000_ICC_20

2000_ICC_21

2003_PDUAI_01

2003_PDUAI_02

2003_PDUAI_03

2007_AFA_01

2007_AFA_02

2007_AFA_03

2007_AFA_04

2007_AFA_05

2007_AFA_06

2007_AFA_07

2007_AFA_08

2007_AFA_09

2007_AFA_10

2008_AFA_01

2008_AFA_02

2008_AFA_03
Riuclar. Vista de una comida popular | Autor Desconocido

Riuclar. Vista de la Horta Gran que rodea al barrio Icomar | Autor Desconocido

Tarragona. Vista del río Francolí | Autor Desconocido

Riuclar. Vista de los alrededores del barrio | Asociación de Vecinos

Puerto. Vista aérea | J. Farré

Puerto. Vista aérea | Altair Photo S.A.

Puerto. Accidente de Robert Maerks en pantalán Repsol | Lluis Penedés

Puerto. Vista aérea de los ejes transversales | Autor Desconocido

Industria. Vista aérea | J. Farré

Tarragona. Riada en el río Francolí | Antonio Martínez Gabarrón

Tarragona. Riada en el río Francolí | Rosa Sans

Tarragona. Riada en el río Francolí | Antonio Martínez Gabarrón

Tarragona. Ortofoto | ICC

Basf. Noticia publicitaria | Autor Desconocido

Noticias de Basf, Repsol y Messer | Autor Desconocido

Polígono químico. Vista aérea | Aviotec

Polígono Entrevías. Vista aérea | Aviotec

Universidad Laboral. Vista aérea | Aviotec

Industrias Químicas Asociadas. Vista aérea | Aviotec

Polígono químico. Vista aérea | Aviotec

Campsa. Vista aérea | Aviotec

Polígono Francolí. Vista aérea | Aviotec

Les Gavarres. Vista aérea | Aviotec

Polígono Entrevías. Vista aérea | Aviotec

El Serrallo. Vista aérea | Aviotec

Polígono Entrevías. Vista aérea | Aviotec

Campclar. Vista aérea | Aviotec

La Pineda. Vista aérea | Aviotec

Les Gavarres. Vista aérea | Aviotec

Tarrgona. Vista aérea | ICC

Puerto. Vista aérea | Técnicas Fotográficas S.L.

Puerto. Vista aérea | Técnicas Fotográficas S.L.

La Canonja. Vista de los alrededores del pueblo | Autor Desconocido

La Canonja. Inundaciones en un campo de avellanos | Autor Desconocido

Tarragona. Plano general | Autor Desconocido

Tarragona. Plano general | GeoEstel

PDUAITCT. Situación | Generalitat de Catalunya

PDUAITCT. Planeamiento vigente | Generalitat de Catalunya

PDUAITCT. Estructura general | Generalitat de Catalunya

Vila-seca. Vista aérea del polígono Industrial | Aviotec

Butano S.A. Vista aérea | Aviotec

Campsa. Vista aérea | Aviotec

Polígono Entrevías. Vista aérea | Aviotec

El polígono químico, el puerto y los barrios del Ponent | Aviotec

Les Gavarres. Vista aérea | Aviotec

Butano S.A. Vista aérea | Aviotec

Puerto. Vista aérea | Aviotec

Puerto. Vista aérea | Aviotec

Puerto. Vista aérea | Aviotec

El cauce del Francolí entre la ciudad y el Ponent | Aviotec

Puerto. Vista aérea de las instalaciones para vehículos | Aviotec

Polígono Entrevías. Vista aérea de las instalaciones Fecsa-Endesa | Aviotec 
2008_APT_04

2009_AFA_01

2009_AFA_02

2009_AFA_03

2010_AFA_01

2010_AFA_02

2010_APT_03

2010_LLC_04

2010_ICC_05

2010_PDUAC_06

2010_PDUAC_07

2010_PDUAC_08

2010_PTCT_09

2011_AAV_01

2011_AEQT_02

2011_AEQT_03

2011_AEQT_04

2011_AFA_05

2011_AFA_06

2011_AFA_07

2011_LLC_08

2011_LLC_09

2011_LLC_10

2011_LLC_11

2011_LLC_12

2011_LLC_13

2011_LLC_14

2011_EMT_15

2011_MFE_16

2012_RFA_01

2012_RFA_02

2012_RFA_03

2012_RFA_04

2012_RFA_05

2012_RFA_06

2012_RFA_07

2012_RFA_08

2012_RFA_09

2012_RFA_10

2012_RFA_11

2012_RFA_12

2012_RFA_13

2012_RFA_14

2012_RFA_15

2012_RFA_16

2012_RFA_17

2012_RFA_18

2012_RFA_19

2012_RFA_20

2012_RFA_21

2012_RFA_22

2012_RFA_23
Puerto. Plano con proyectos de ampliación del puerto | Puerto de Tarragona Vista aérea de Ponent de Tarragona y la N-340 | Aviotec Bonavista. Vista área del mercado dominical | Aviotec Polígono Francolí. Vista área | Aviotec Puerto. Vista aérea | Aviotec

Polígono Francolí. Vista aérea | Aviotec

Puerto. Plan Director | Puerto de Tarragona

La Canonja. Plano del término municipal | Autor Desconocido

Tarragona. Ortofoto | ICC

PDUAC. Polígono Industrial Sur | Generalitat de Catalunya

PDUAC. Parque Fluvial del Francolí| Generalitat de Catalunya

PDUAC. Ordenación de asentamientos urbanos|Generalitat de Catalunya

PTCT. Estrategias asentamiento y infraestructuras|Generalitat de Catalunya

Raval de Mar de Vila-seca. Vista aérea | Autor Desconocido

Petroquímica. Vista aérea del sector Norte | Autor Desconocido

Polígono Entrevías. Vista aérea | Arquitecto Ripoll

Industria. Vista aérea | Autor Desconocido

Polígono químico. Vista aérea | Aviotec

Polígono químico. Vista aérea | Aviotec

Polígono químico. Vista aérea $\mid$ Aviotec

La Canonja. Vista del pueblo y de la industria | Autor Desconocido

La Canonja. Vista del pueblo y de la industria | Autor Desconocido

La Canonja. Vista del pueblo y de la industria | Autor Desconocido

La Canonja. Vista aérea del pueblo y de la industria | Autor Desconocido

La Canonja. Vista de la industria | Autor Desconocido

La Canonja. Vista aérea del pueblo y de la industria | Autor Desconocido

La Canonja. Vista aérea del pueblo y de la industria | Autor Desconocido

Tarragona. Plano líneas de autobús | EMT

Estudio del corredor ferroviario mediterráneo | Ineco

Tarragona. Vista de la bajada al río Francolí | Juan Manuel Zaguirre

Tarragona. Vista del río Francolí | Juan Manuel Zaguirre

Tarragona. Conductos junto al río Francolí | Juan Manuel Zaguirre

Tarragona. Muros entre el río Francolí y Campsa | Juan Manuel Zaguirre

Tarragona. Muros entre el río Francolí y Industria | Juan Manuel Zaguirre

Conductos del rack en el cauce del Francolí | Juan Manuel Zaguirre

Tarragona. Vista del río Francolí | Juan Manuel Zaguirre

Tarragona. Vista del río Francolí | Juan Manuel Zaguirre

Tarragona. Vista del río Francolí | Juan Manuel Zaguirre

Tarragona. Vista del río Francolí | Juan Manuel Zaguirre

Tarragona. Muros entre el río Francolí y la ciudad | Juan Manuel Zaguirre Tarragona. Vista del río Francolí | Juan Manuel Zaguirre

Tarragona. Puentes ferroviarios sobre el Francolí | Juan Manuel Zaguirre Tarragona. Vista de desembocadura al río Francolí | Juan Manuel Zaguirre Tarragona. Vista de desembocadura al río Francolí | Juan Manuel Zaguirre Tarragona. Puentes ferroviarios sobre el Francolí| Juan Manuel Zaguirre Tarragona. Puentes ferroviarios sobre el Francolí| Juan Manuel Zaguirre Tarragona. Puentes ferroviarios sobre el Francolí| Juan Manuel Zaguirre Tarragona. Puentes ferroviarios sobre el Francolí | Juan Manuel Zaguirre Tarragona. Puentes ferroviarios sobre el Francolí| Juan Manuel Zaguirre Tarragona. Puentes ferroviarios sobre el Francolí| Juan Manuel Zaguirre Tarragona. Vista cartel Ematsa-Medi Ambient | Juan Manuel Zaguirre Tarragona. Puentes ferroviarios sobre el Francolí | Juan Manuel Zaguirre
2012_RFA_24

2012_RFA_25

2012_RFA_26

2012_RFA_27

2012_RFA_28

2012_RFA_29

2012_RFA_30

2012_RFA_31

2012_RFA_32

2012_RFA_33

2012_RFA_34

2012_RFA_35

2012_RFA_36

2012_RFA_37

2012_RFA_38

2012_RFA_39

2012_RFA_40

2012_RFA_41

2012_RFA_42

2012_RFA_43

2012_RFA_44

2012_RFA_45

2012_RFA_46

2012_RFA_47

2012_RFA_48

2012_RFA_49

2012_RFA_50

2012_RFA_51

2012_RFA_52

2012_RFA_53

2012_RFA_54

2012_RFA_55

2012_RFA_56

2012_RFA_57

2012_RFA_58

2012_RFA_59

2012_RFA_60

2012_RFA_61

2012 RFA 62

2012_RFA_63

2012_RFA_64

2012_RFA_65

2012_RFA_66

2012_RFA_67

2012_RFA_68

2012_RFA_69

2012_RFA_70

2012 RFA_71

2012_RFA_72

2012_RFA_73

2012_RFA_74

2012_RFA_75
Tarragona. Puentes ferroviarios sobre el Francolí | Juan Manuel Zaguirre Tarragona. Puentes ferroviarios sobre el Francolí | Juan Manuel Zaguirre Tarragona. Puentes ferroviarios sobre el Francolí | Juan Manuel Zaguirre Tarragona. Vista del río Francolí | Juan Manuel Zaguirre

Tarragona. Vista de la parte baja del río Francolí | Juan Manuel Zaguirre Tarragona. Club de piragüismo en el río Francolí | Juan Manuel Zaguirre Tarragona. Club de piragüismo en el río Francolí | Juan Manuel Zaguirre Tarragona. Club de piragüismo en el río Francolí | Juan Manuel Zaguirre Tarragona. Club de piragüismo en el río Francolí | Juan Manuel Zaguirre Tarragona. Club de piragüismo en el río Francolí | Juan Manuel Zaguirre Tarragona. Club de piragüismo en el río Francolí | Juan Manuel Zaguirre Tarragona. Club de piragüismo en el río Francolí | Juan Manuel Zaguirre Tarragona. Muros entre el río Francolí y la ciudad | Juan Manuel Zaguirre Tarragona. Muros entre el río Francolí y la ciudad | Juan Manuel Zaguirre Tabacalera. Vista de la fachada | Juan Manuel Zaguirre Polígono Francolí. Vista de una vía principal | Juan Manuel Zaguirre Polígono Francolí. Vista de una vía principal | Juan Manuel Zaguirre Polígono Francolí. Vista de las naves | Juan Manuel Zaguirre Polígono Francolí. Vista de las naves | Juan Manuel Zaguirre Polígono Francolí. Vista de una vía principal | Juan Manuel Zaguirre Polígono Francolí. Vista de las naves | Juan Manuel Zaguirre Icomar. Vista de un edificio de viviendas | Juan Manuel Zaguirre Polígono Francolí. Vista de una vía principal | Juan Manuel Zaguirre Polígono Francolí. Vista de las naves | Juan Manuel Zaguirre Polígono Francolí. Vista de las naves | Juan Manuel Zaguirre Polígono Francolí. Vista de las naves | Juan Manuel Zaguirre Polígono Francolí. Vista de las naves | Juan Manuel Zaguirre Polígono Francolí. Vista de las naves | Juan Manuel Zaguirre Polígono Francolí. Vista de las naves | Juan Manuel Zaguirre Polígono Francolí. Vista de las naves | Juan Manuel Zaguirre Polígono Francolí. Vista de las naves | Juan Manuel Zaguirre Polígono Francolí. Vista de las naves | Juan Manuel Zaguirre Polígono Francolí. Vista de las naves | Juan Manuel Zaguirre Polígono Francolí. Vista de las naves | Juan Manuel Zaguirre Polígono Francolí. Vista de los alrededores | Juan Manuel Zaguirre Ferrocarril. Vista de las vías | Juan Manuel Zaguirre Ferrocarril. Vista de las vías | Juan Manuel Zaguirre Ferrocarril. Vista de las vías | Juan Manuel Zaguirre Polígono Francolí. Vista de los alrededores | Juan Manuel Zaguirre Vista de barracas que persisten en Entrevías | Juan Manuel Zaguirre Polígono Francolí. Vista de los alrededores | Juan Manuel Zaguirre Vista de barracas que persisten en Entrevías | Juan Manuel Zaguirre Polígono Francolí. Vista de los alrededores | Juan Manuel Zaguirre Vista de barracas que persisten en Entrevías | Juan Manuel Zaguirre Vista de barracas que persisten en Entrevías | Juan Manuel Zaguirre Ferrocarril. Vista de las vías | Juan Manuel Zaguirre Ferrocarril. Estación bajo un puente y de las vías | Juan Manuel Zaguirre Vista de barracas que persisten en Entrevías | Juan Manuel Zaguirre Vista de barracas que persisten en Entrevías | Juan Manuel Zaguirre Ferrocarril. Vista de las vías | Juan Manuel Zaguirre Ferrocarril. Vista de las vías | Juan Manuel Zaguirre Ferrocarril. Vista de las vías | Juan Manuel Zaguirre 
2012_RFA_76 Polígono Francolí. Vista de los alrededores | Juan Manuel Zaguirre

2012_RFA_77 Polígono Francolí. Vista de las naves | Juan Manuel Zaguirre

2012_RFA_78 Ferrocarril. Vista de las vías | Juan Manuel Zaguirre

2012_RFA_79 Ferrocarril. Vista de las vías | Juan Manuel Zaguirre

2012_RFA_80 N-340. Vista de una nave | Juan Manuel Zaguirre

2012_RFA_81 N-340. Vista del tramo que conserva los plataneros| Juan Manuel Zaguirre

2012_RFA_82 N-340. Vista del tramo que conserva los plataneros | Juan Manuel Zaguirre

2012_RFA_83 Polígono Francolí. Vista de los alrededores | Juan Manuel Zaguirre

2012_RFA_84 Polígono Francolí. Vista de los alrededores | Juan Manuel Zaguirre

2012_RFA_85 Polígono Francolí. Vista de los alrededores | Juan Manuel Zaguirre

2012_RFA_86 Polígono Francolí. Vista de los alrededores | Juan Manuel Zaguirre

2012_RFA_87 Polígono Francolí. Vista de los alrededores | Juan Manuel Zaguirre

2012_RFA_88 Polígono Francolí. Vista de los alrededores | Juan Manuel Zaguirre

2012_RFA_89 Polígono Francolí. Vista de los alrededores | Juan Manuel Zaguirre

2012_RFA_90 Polígono Francolí. Vista de los alrededores | Juan Manuel Zaguirre

2012_RFA_91 Polígono Francolí. Vista de los alrededores | Juan Manuel Zaguirre

2012_RFA_92 Polígono Francolí. Vista de los alrededores | Juan Manuel Zaguirre

2012_RFA_93 Asesa. Cartel | Juan Manuel Zaguirre

2012_RFA_94 Viaducto del eje transversal sobre Entrevías-Clasificación | Juan Manuel Zaguirre

2012_RFA_95 Polígono Industrial. Vista de barracas | Juan Manuel Zaguirre

2012_RFA_96 Polígono Francolí. Vista de los alrededores | Juan Manuel Zaguirre

2012_RFA_97 Polígono Industrial. Vista de barracas | Juan Manuel Zaguirre

2012_RFA_98 Messer. Vista de un marcador | Juan Manuel Zaguirre

2012_RFA_99 Imagen del “intraestructuralismo” en Entrevías | Juan Manuel Zaguirre

2012_RFA_100 Imagen del “intraestructuralismo” en Entrevías | Juan Manuel Zaguirre

2012_RFA_101 Imagen del “intraestructuralismo” en Entrevías | Juan Manuel Zaguirre

2012_RFA_102 Imagen del "intraestructuralismo" en Entrevías | Juan Manuel Zaguirre

2012_RFA_103 Polígono Entrevías. Vista de los alrededores | Juan Manuel Zaguirre

2012_RFA_104 Polígono Entrevías. Vista de los alrededores | Juan Manuel Zaguirre

2012_RFA_105 Polígono Entrevías. Vista de los alrededores | Juan Manuel Zaguirre

2012_RFA_106 Polígono Entrevías. Vista de los alrededores | Juan Manuel Zaguirre

2012_RFA_107 Polígono Entrevías. Vista de los alrededores | Juan Manuel Zaguirre

2012_RFA_108 Polígono Entrevías. Vista de unos contenedores | Juan Manuel Zaguirre

2012_RFA_109 Polígono Entrevías. Vista de los alrededores | Juan Manuel Zaguirre

2012_RFA_110 Polígono Entrevías. Vista de las naves | Juan Manuel Zaguirre

2012_RFA_111 Polígono Entrevías. Vista de maquinaria | Juan Manuel Zaguirre

2012_RFA_112 Polígono Entrevías. Vista de unos contenedores | Juan Manuel Zaguirre

2012_RFA_113 Aragonesas. Vista de un marcador | Juan Manuel Zaguirre

2012_RFA_114 Ferrocarril. Vista de las vías | Juan Manuel Zaguirre

2012_RFA_115 Polígono Entrevías. Vista de marcador de cables AT | Juan Manuel Zaguirre

2012_RFA_116 Polígono Entrevías. Vista de las naves | Juan Manuel Zaguirre

2012_RFA_117 Polígono Entrevías. Vista de las naves | Juan Manuel Zaguirre

2012_RFA_118 Polígono Entrevías. Vista de las naves | Juan Manuel Zaguirre

2012_RFA_119 Polígono Entrevías. Vista de las naves | Juan Manuel Zaguirre

2012_RFA_120 Polígono Entrevías. Vista aérea | Juan Manuel Zaguirre

2012_RFA_121 Polígono químico. Vista de la industria | Juan Manuel Zaguirre

2012_RFA_122 Polígono químico. Vista de la industria | Juan Manuel Zaguirre

2012_RFA_123 Polígono químico. Vista de la industria | Juan Manuel Zaguirre

2012_RFA_124 Ferrocarril. Vista de una vía en polígono Entrevías | Juan Manuel Zaguirre

2012_RFA_125 Ferrocarril. Vista de una vía en polígono Entrevías | Juan Manuel Zaguirre

2012_RFA_126 Polígono químico. Vista de la autovía de Salou | Juan Manuel Zaguirre

2012_RFA_127 Ferrocarril. Vista de una vía en polígono Entrevías | Juan Manuel Zaguirre
2012_RFA_128

2012_RFA_129

2012_RFA_130

2012_RFA_131

2012_RFA_132

2012_RFA_133

2012_RFA_134

2012_RFA_135

2012_RFA_136

2012_RFA_137

2012_RFA_138

2012_RFA_139

2012_RFA_140

2012_RFA_141

2012_RFA_142

2012_RFA_143

2012_RFA_144

2012_RFA_145

2012_RFA_146

2012_RFA_147

2012_RFA_148

2012_RFA_149

2012_RFA_150

2012_RFA_151

2012_RFA_152

2012_RFA_153

2012_RFA_154

2012_RFA_155

2012_RFA_156

2013_RFA_157

2013_RFA_158

2013_RFA_159

2013_RFA_160

2013_RFA_161

2013_RFA_162

2013_RFA_163

2013_RFA_164

2013_RFA_165

2013_RFA_166

2013_RFA_167

2013_RFA_168

2014_RFA_169

2014_RFA_170

2014_RFA_171

2014_RFA_172

2014_RFA_173

2014_RFA_174

2014_RFA_175

2014_RFA_176

2014_RFA_177

2014_RFA_178

2014_RFA_179
Polígono químico. Vista de la industria | Juan Manuel Zaguirre Polígono químico. Vista de la industria | Juan Manuel Zaguirre Polígono químico. Vista de la industria | Juan Manuel Zaguirre Polígono químico. Vista de la industria | Juan Manuel Zaguirre Campos y factorías químicas | Juan Manuel Zaguirre Polígono químico. Vista de la industria | Juan Manuel Zaguirre Polígono químico. Vista de la industria | Juan Manuel Zaguirre Campos y depósitos de combustible | Juan Manuel Zaguirre Polígono químico. Vista de la industria | Juan Manuel Zaguirre Polígono químico. Vista de la industria | Juan Manuel Zaguirre Polígono químico. Vista de la industria | Juan Manuel Zaguirre Polígono químico. Vista de la industria | Juan Manuel Zaguirrev Polígono químico. Vista de la industria | Juan Manuel Zaguirre Polígono químico. Vista de la industria | Juan Manuel Zaguirre $\mathrm{N}$-340. Vista de la carretera en el barrio Campclar | Juan Manuel Zaguirre N-340 a su paso por Bonavista en dirección a Vila-seca | Juan Manuel Zaguirre Polígono químico. Vista de la industria | Juan Manuel Zaguirre Polígono químico. Vista de los alrededores | Juan Manuel Zaguirree Polígono químico. Vista de la industria | Juan Manuel Zaguirre Polígono químico. Vista de los alrededores | Juan Manuel Zaguirre Carretera La Pineda - Tarragona. Antiguo Camí Vell de Salou | Juan Manuel Zaguirre Polígono químico. Vista de los alrededores | Juan Manuel Zaguirre Polígono químico. Vista de los alrededores | Juan Manuel Zaguirre Carretera La Pineda - Tarragona. Antiguo Camí Vell de Salou |Juan Manuel Zaguirre Polígono químico. Vista de los alrededores | Juan Manuel Zaguirre Polígono químico. Vista de los alrededores | Juan Manuel Zaguirre Polígono químico. Vista de la carretera de Salou | Juan Manuel Zaguirre Polígono químico. Vista de la industria | Juan Manuel Zaguirre Polígono químico. Vista de la industria | Juan Manuel Zaguirre A-27 en construcción. Prolongación del eje transversal | Juan Manuel Zaguirre A-27 en construcción. Prolongación del eje transversal | Juan Manuel Zaguirre Construcción de la A-27 | Juan Manuel Zaguirre Construcción de la A-27 | Juan Manuel Zaguirre Construcción de la A-27 | Juan Manuel Zaguirre A-7. Vista a su paso por Vila-seca | Juan Manuel Zaguirre Vila-seca. Vista del Raval de Mar | Juan Manuel Zaguirre Vista del Raval de Mar entre Vila-seca y La Pineda | Juan Manuel Zaguirre Vila-seca. Vista del Raval de Mar | Juan Manuel Zaguirre Vila-seca. Vista del puente peatonal sobre la A-7 | Juan Manuel Zaguirre Vila-seca. Vista del Raval de Mar | Juan Manuel Zaguirre Vila-seca. Vista del Raval de Mar | Juan Manuel Zaguirre Tarragona. Vista panorámica de la ciudad | Juan Manuel Zaguirre Tarragona. Vista general | Juan Manuel Zaguirre Tarragona. Vista de los alrededores | Juan Manuel Zaguirre Las acequias de la Horta Gran | Juan Manuel Zaguirre Acequias de la Horta Gran | Juan Manuel Zaguirre Acequias de la Horta Gran | Juan Manuel Zaguirre Riuclar. Vista de los alrededores del barrio | Juan Manuel Zaguirre Riuclar. Vista de la riera | Juan Manuel Zaguirre

Riuclar. Vista de los alrededores del barrio | Juan Manuel Zaguirre Riuclar. Vista de los alrededores del barrio | Juan Manuel Zaguirre Riuclar. Vista de los alrededores del barrio | Juan Manuel Zaguirre 
2014_RFA_180

La Pineda. Vista de la playa | Juan Manuel Zaguirre

2014_RFA_181 La Pineda. Vista de la playa | Juan Manuel Zaguirre

2014_RFA_182

2014_RFA_183

2014_RFA_184

2014_RFA_185

2014_RFA_186

2014_RFA_187

2014_RFA_188

2014_RFA_189

2014_RFA_190

2014_RFA_191

2014_RFA_192

2014_RFA_193

La Pineda. Vista de la playa | Juan Manuel Zaguirre

La Pineda. Vista del espigón | Juan Manuel Zaguirre

La Pineda. Vista de la playa | Juan Manuel Zaguirre

La Pineda. Vista de la playa | Juan Manuel Zaguirre

La Pineda. Vista de los alrededores de la playa | Juan Manuel Zaguirre

La Pineda. Vista de los alrededores de la playa | Juan Manuel Zaguirre

La Pineda. Vista de los alrededores de la playa | Juan Manuel Zaguirre

La Pineda. Vista de los alrededores de la playa | Juan Manuel Zaguirre

La Pineda. Vista de los alrededores de la playa | Juan Manuel Zaguirre

La Pineda. Vista de los alrededores de la playa | Juan Manuel Zaguirre

Campclar. Vista del barrio desde la T-II | Juan Manuel Zaguirre

2014_RFA_194

T-II. Vista a la altura de Les Gavarres | Juan Manuel Zaguirre

MEP_AA_1956_20 Ortofoto modificado. Núcleos urbanos consolidados en 1956 | J.M.Z./E.A.

MEP_AM_1930_01 Morfogénesis 1930|J.M.Z./E.A.

MEP_AM_1952_02 Morfogénesis 1952 | J.M.Z./E.A.

MEP_AM_1956_03 Morfogénesis 1956 | J.M.Z./E.A.

MEP_AM_1964_04 Morfogénesis 1964 |J.M.Z./E.A.

MEP_AM_1983_05 Morfogénesis 1983 | J.M.Z./E.A.

MEP_AM_1996_06 Morfogénesis 1996 | J.M.Z./E.A.

MEP_AM_2009_07 Morfogénesis 2009 | J.M.Z./E.A.

MEP_AM_2016_08 Morfogénesis 20I6. Infraestructuras | J.M.Z./E.A.

MEP_AP_1932_01 Secuencia de 1932. Topografía |J.M.Z./E.A.

MEP_AP_1932_02 Secuencia de 1932. Hidrografía | J.M.Z./E.A.

MEP_AP_1932_03 Secuencia de 1932. Red de caminos principales |J.M.Z./E.A.

MEP_AP_1932_04 Secuencia de I932. Parcelación urbana |J.M.Z./E.A.

MEP_AP_1932_05 Secuencia de I932. Parcelación rústica de regadío |J.M.Z./E.A.

MEP_AP_1932_06 Secuencia de 1932. Parcelación rústica de secano |J.M.Z./E.A.

MEP_AP_1932_07 Secuencia de 1932. Parcelación rústica de costa |J.M.Z./E.A.

MEP_AP_1932_08 Secuencia de I932. Reparcelación | J.M.Z./E.A.

MEP_AP_1932_09 Parcelas con superficie inferior a $10.000 \mathrm{~m}^{2} \mid$ J.M.Z./E.A.

MEP_AP_1932_10 Parcelas con superficie entre 10.000 y $50.000 \mathrm{~m}^{2}$ |J.M.Z./E.A.

MEP_AP_1932_11 Parcelas con superficie entre 50.000 y $100.000 \mathrm{~m}^{2}$ | J.M.Z./E.A.

MEP_AP_1932_12 Parcelas con superficie superior a $100.000 \mathrm{~m}^{2}$ |J.M.Z./E.A.

MEP_AP_1932_13 Parcelas ocupadas |J.M.Z./E.A.

MEP_AR_2010_01 Ortofoto modificada. Edificado en Entrevías | J.M.Z./E.A.

MEP_AR_2010_02 Ortofoto modificada."Industrialización marginal”en Entrevías|J.M.Z./E.A.

MEP_AR_2010_03 Ortofotoplano modificado. "No urbanizado" en Entrevías | J.M.Z./E.A.

MEP_AR_1983_04 Plano modificado. Edificación no industrial en Entrevías | J.M.Z./E.A.

MEP_AR_1983_05 Plano modificado. "Industrialización marginal” en Entrevías |J.M.Z./E.A.

MEP_AR_1983_06 Plano modificado. Racks en Entrevías | J.M.Z./E.A.

MEP_AR_1983_07 Plano modificado."No urbanizado” en Entrevías | J.M.Z./E.A.

MEP_N_2010_17 Sección longitudinal por N-340 | J.M.Z./E.A.

MEP_N_2010_18 Nudos y conexiones en la N-340 |J.M.Z./E.A.

MEP_N_1807_01 Secuencia cartográfica de crecimiento en la N-34O, I807 | J.M.Z./E.A.

MEP_N_1855_02 Secuencia cartográfica de crecimiento en la N-34O, I855 |J.M.Z./E.A.

MEP_N_1860_03 Secuencia cartográfica de crecimiento en la N-340, I860 | J.M.Z./E.A.

MEP_N_1876_04 Secuencia cartográfica de crecimiento en la N-340, I876 | J.M.Z./E.A.

MEP_N_1882_05 Secuencia cartográfica de crecimiento en la N-340, I882 | J.M.Z./E.A.

MEP_N_1919_06 Secuencia cartográfica de crecimiento en la N-340, I9I9 | J.M.Z./E.A.
MEP_N_1922_07 Secuencia cartográfica de crecimiento en la N-34O, I922 |J.M.Z./E.A. MEP_N_1939_08 Secuencia cartográfica de crecimiento en la N-340, I939 | J.M.Z./E.A. MEP_N_1956_09 Secuencia cartográfica de crecimiento en la N-340, 1956 | J.M.Z./E.A. MEP_N_1960_10 Secuencia cartográfica de crecimiento en la N-340, 1960 | J.M.Z./E.A. MEP_N_1966_11 Secuencia cartográfica de crecimiento en la N-340, I966 | J.M.Z./E.A. MEP_N_1973_12 Secuencia cartográfica de crecimiento en la N-340, I973 | J.M.Z./E.A. MEP_N_1983_13 Secuencia cartográfica de crecimiento en la N-340, I983 | J.M.Z./E.A. MEP_N_1996_14 Secuencia cartográfica de crecimiento en la N-340, I996 | J.M.Z./E.A. MEP_N_2009_15 Secuencia cartográfica de crecimiento en la N-340, 2009 | J.M.Z./E.A. MEP_N_2016_16 Secuencia cartográfica de crecimiento en la N-34O, 20I6 |J.M.Z./E.A. MEP_N_2010_19 La Gran Vía del Camp. N-340 y la Gran Vía de Barcelona | J.M.Z./E.A. MEP_P_1801_01 Secuencia cartográfica de crecimiento portuario, I80I | J.M.Z./E.A. MEP_P_1807_02 Secuencia cartográfica de crecimiento portuario, I807 | J.M.Z./E.A. MEP_P_1855_03 Secuencia cartográfica de crecimiento portuario, I855 | J.M.Z./E.A. MEP_P_1860_04 Secuencia cartográfica de crecimiento portuario, I860 | J.M.Z./E.A. MEP_P_1876_05 Secuencia cartográfica de crecimiento portuario, 1876 | J.M.Z./E.A. MEP_P_1882_06 Secuencia cartográfica de crecimiento portuario, I882 |J.M.Z./E.A. MEP_P_1919_07 Secuencia cartográfica de crecimiento portuario, I9I9 | J.M.Z./E.A. MEP_P_1928_08 Secuencia cartográfica de crecimiento portuario, I928 | J.M.Z./E.A. MEP_P_1939_09 Secuencia cartográfica de crecimiento portuario, I939 |J.M.Z./E.A. MEP_P_1956_10 Secuencia cartográfica de crecimiento portuario, I956 | J.M.Z./E.A. MEP_P_1976_11 Secuencia cartográfica de crecimiento portuario, I976 | J.M.Z./E.A. MEP_P_1983_12 Secuencia cartográfica de crecimiento portuario, I983P | J.M.Z./E.A. MEP_P_1983_13 Secuencia cartográfica de crecimiento portuario, I983 | J.M.Z./E.A. MEP_P_1996_14 Secuencia cartográfica de crecimiento portuario, 1996 | J.M.Z./E.A. MEP_P_2009_15 Secuencia cartográfica de crecimiento portuario, 2009 |J.M.Z./E.A. MEP_P_2016_16 Secuencia cartográfica de crecimiento portuario, 20I6 |J.M.Z./E.A. MEP_P_2010_18 Entre el mar y Entrevías. El puerto |J.M.Z./E.A.

MEP_P_1992_17 Superposición de evolución del ámbito portuario. 1992-2004 | J.M.Z./E.A. MEP_R_1960_01 Superposición del Plan Monravà de ig60 y actuales |J.M.Z./E.A.

MEP_R_1973_02 Superposición del Plan de Infraestructuras de I973 y actuales | J.M.Z./E.A. MEP_R_1960-83_03 Superposición de un plano del PGO de I960 con ortofotografía de I983 MEP_R_1973-83_04 Coincidencias entre el esquema viario de I973 y el plan general de I983 MEP_R_1973-10_05 Superposición del esquema viario de I973 con ortofotografía de 20I0 MEP_R_1974-83_06 Superposición del cinturón (2.ffi fase) con ortofoto de I983 | J.M.Z./E.A. MEP_U_2010_01 Diferentes urbanidades en Entrevías | J.M.Z./E.A.

MEP_T_1851_01 Plano de la línea de tren de Reus-Tarragona | J.M.Z.

MEP_T_1854_03 Plano de la línea de tren de Tarragona-Barcelona | J.M.Z.

MEP_T_1855_04 Plano de la línea de tren de Valencia-Tarragona |J.M.Z.

MEP_T_1856_05 Plano de la línea de tren de Reus-Montblanc|J.M.Z.

MEP_T_1857_07 Plano de la línea de tren de Lérida-Tarragona|J.M.Z.

MEP_T_1862_08 Plano de la línea de tren de Reus-Tarragona-Martorell-Barcelona |J.M.Z.

MEP_T_1884_09 Plano de la línea de tren de Reus - Roda de Bará | J.M.Z.

MEP_T_2006_11 Plano de las vías de tren en 20io con vías en proyecto | J.M.Z.

MEP_V_1807_01 Secuencia viaria. Proyecto del canal de Reus | J.M.Z.

MEP_V_1855_02 Secuencia viaria. Proyecto trazado Tarragona-Reus |J.M.Z.

MEP_V_1956_03 Secuencia viaria. Estado en 1956 | J.M.Z.

MEP_V_1960_04 Secuencia viaria. Plan General de Ordenación de Ig60|J.M.Z.

MEP_V_1963_05 Secuencia viaria. Plan Especial Ordenación Costa de Tarragona |J.M.Z.

MEP_V_1967_06 Secuencia viaria. Plan Comarcal de I967|J.M.Z.

MEP_V_1980_07 Secuencia viaria. Comisión de Municipios del Camp de I980 | J.M.Z.

MEP_V_1983_08 Secuencia viaria. Plan General Ordenación Urbana de Tarragona|J.M.Z. 

1. - 6)
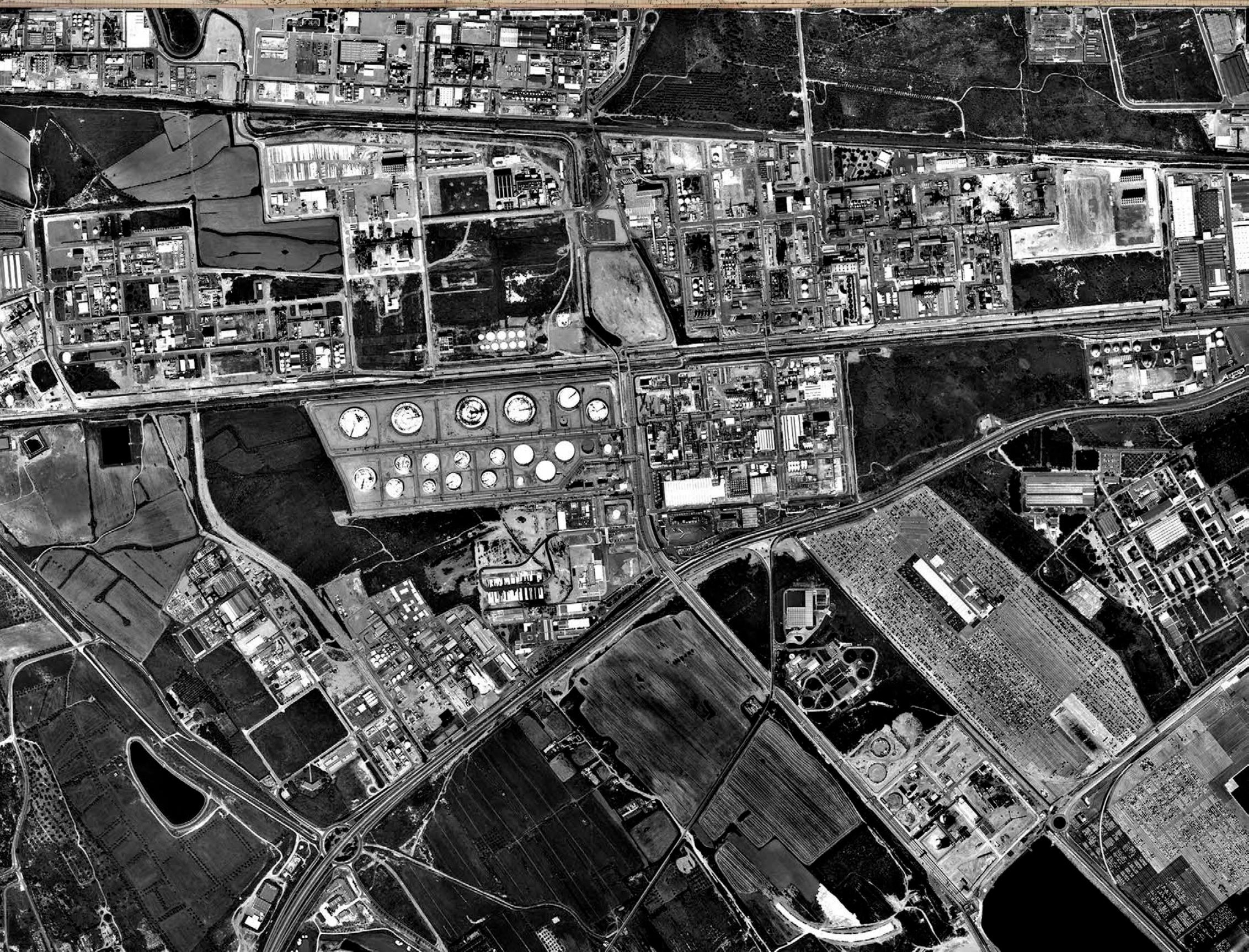